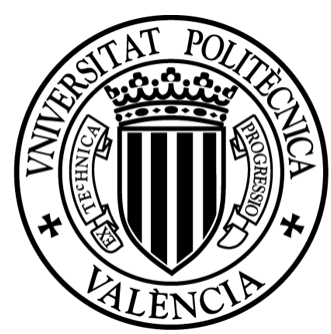

UNIVERSIDAD POLITÉCNICA DE VALENCIA DePartamento de Matemática APlicada

\title{
FINITE DIFFERENCE METHODS FOR NONLINEAR AMERICAN OPTION PRICING MODELS: NUMERICAL ANALYSIS AND COMPUTING
}

\author{
Tesis doctoral presentada por Vera Egorova \\ dentro del Programa de Doctorado en Matemáticas \\ Dirigida por Dr. Lucas Jódar Sánchez \\ y Dr. Rafael Company Rossi
}

El doctorando

El director

El director 
Finite Difference Methods for nonlinear American Option Pricing models: Numerical Analysis and Computing

Author: Vera Nikolaevna Egorova

Advisor: Dr. Lucas Jódar Sánchez

Advisor: Dr. Rafael Company Rossi

Text printed in Valencia

Third edition, April 2016 


\section{Resumen}

La presente tesis doctoral se centra en la construcción de esquemas en diferencias finitas y el análisis numérico de relevantes modelos de valoración de opciones que generalizan el modelo de Black-Scholes. Se proporciona un análisis cuidadoso de las propiedades de las soluciones numéricas tales como la positividad, la estabilidad y la consistencia.

Con el fin de manejar la frontera libre que surge en los problemas de valoración de opciones Americanas, se aplican y se estudian diversas técnicas de transformación basadas en el método de fijación de las fronteras (front-fixing). Se presta especial atención a la valoración de opciones de múltiples activos, como son las opciones "exchange" y "spread". Se propone una transformación apropiada que permite la eliminación del término de derivadas cruzadas. Se estudian también técnicas de transformación de las ecuaciones diferenciales en derivadas parciales para eliminar términos de convección y de reacción con el propósito de simplificar los modelos y evitar posibles problemas de estabilidad.

Esta tesis se compone de seis capítulos. El primer capítulo es una introducción que contiene las definiciones de opción y términos relacionados y la derivación de la ecuación de Black-Scholes, así como aspectos generales de la teoría de los esquemas en diferencias finitas, incluyendo preliminares de análisis numérico.

El capítulo 2 está dedicado a resolver el modelo lineal de Black-Scholes para opciones Americanas put y call. Para fijar las fronteras del problema de frontera libre se aplican transformaciones como la de Landau y un nuevo cambio de variable propuesto. Esto lleva a una ecuación diferencial en derivadas parciales no lineal en un dominio fijo. Se proponen esquemas numéricos explícitos estables y consistentes que preservan la positividad y monotonicidad de la solución de acuerdo con el comportamiento de la solución exacta. 
La eficiencia del método front-fixing mostrada en el capítulo 2 ha motivado el estudio de su aplicación a algunos modelos no lineales más complicados. En particular, se propone un cambio de variables que lleva a una nueva frontera dependiente del tiempo en lugar de una fija. Este cambio se aplica a modelos no lineales de Black-Scholes para opciones Americanas, como son el de Barles y Soner y el modelo RAPM (Risk Adjusted Pricing Methodology). Se construye un algoritmo numérico eficiente para el caso general de volatilidad no constante en la sección 3.1. Con el fin de resolver la ecuación, se proponen varios métodos de diferencias finitas, incluyendo métodos explícitos, implícitos y ADE (Alternating Direction Explicit). Se proponen asimismo nuevas modificaciones del método de Newton para la solución de los sistemas no lineales derivados. En la sección 3.2 se aplica por primera vez el método front-fixing a un problema de valoración de opciones con cambio de regímenes. Dado que en este modelo hay varios regímenes, aparecen varias fronteras libres por lo que la transformación de variables lleva a un sistema de ecuaciones no lineales. Este sistema se resuelve con un esquema en diferencias finitas explícito. Mediante el enfoque de Von Neumann se prueba la estabilidad del esquema.

El capítulo 4 ofrece una nueva técnica para la resolución de problemas de valoración de opciones Americanas basada en la racionalidad de los inversores. Aparece una función de la intensidad que se puede reducir en el caso más simple a la técnica de penalización (penalty method). Este enfoque tiene en cuenta el posible comportamiento irracional de los inversores. En la sección 4.2 se aplica esta técnica al modelo de cambio de regímenes lo que lleva a un nuevo modelo que tiene en cuenta el posible ejercicio irracional, así como varios estados del mercado. El enfoque del parámetro de racionalidad junto con una transformación logarítmica permiten construir un esquema numérico eficiente sin aplicar el método front-fixing o la conocida formulación de LCP (Linear Complementarity Problem). Se propone una familia de esquemas ponderados tanto para opciones americanas de tipo vanilla como para el modelo de cambio de regímenes. Se estudian las propiedades cualitativas de la función de intensidad y de las soluciones numéricas. 
El capítulo 5 se dedica a la valoración de opciones de activos múltiples. Una transformación apropiada permite la eliminación del término de derivadas cruzadas evitando inconvenientes computacionales y posibles problemas de estabilidad.

Las conclusiones se muestran en el capítulo 6. Se pone en relieve varios aspectos de la presente tesis. Todos los modelos considerados y los métodos numéricos van acompañados de varios ejemplos y simulaciones. Se estudia la convergencia numérica que confirma el estudio teórico de la consistencia. Las condiciones de estabilidad son corroboradas con ejemplos numéricos. Los resultados se comparan con métodos relevantes de la bibliografía mostrando la eficiencia de los métodos propuestos. 



\section{Resum}

La present tesi doctoral se centra en la construcció d'esquemes en diferències finites i l'anàlisi numèrica de rellevants models de valoració d'opcions que generalitzen el model de Black-Scholes. Es proporciona una anàlisi cuidadosa de les propietats de les solucions numèriques com ara la positivitat, l'estabilitat i la consistència.

A fi de manejar la frontera lliure que sorgix en els problemes de valoració d'opcions Americanes, s'apliquen i s'estudien diverses tècniques de transformació basades en el mètode de fixació de les fronteres (frontfixing). Es presta especial atenció a la valoració d'opcions de múltiples actius, com són les opcions "exchange" i "spread". Es proposa una transformació apropiada que permet l'eliminació del terme de derivades croades. S'estudien també tècniques de transformació de les equacions diferencials en derivades parcials per a eliminar termes de convecció i de reacció amb el propòsit de simplificar els models i evitar possibles problemes d'estabilitat.

Esta tesi es compon de sis capítols. El primer capítol és una introducció que conté les definicions d'opció i termes relacionats i la derivació de l'equació de Black-Scholes, així com aspectes generals de la teoria dels esquemes en diferències finites, incloent aspectes preliminars d'anàlisi numèrica.

El 2n capítol està dedicat a resoldre el model lineal de Black-Scholes per a opcions Americanes "put" i "call". Per a fixar les fronteres del problema de frontera lliure s'apliquen transformacions com la de Landau i s'ha proposat un nou canvi de variable proposat. Açò porta a una equació diferencial en derivades parcials no lineal en un domini fix. Es proposen esquemes numèrics explícits estables i consistents que preserven la positivitat i monotonia de la solució d'acord amb el comportament de la solució exacta. 
L'eficiència del mètode front-fixing mostrada en el 2 n capítol ha motivat l'estudi de la seua aplicació a alguns models no lineals més complicats. En particular, es proposa un canvi de variables que porta a una nova frontera dependent del temps en compte d'una fixa. Este canvi s'aplica a models no lineals de Black-Scholes per a opcions Americanes, com són el de Barles i Soner i el model RAPM (Risk Adjusted Pricing Methodology). Es construïx un algoritme numèric eficient per al cas general de volatilitat no constant en la secció 3.1. A fi de resoldre l'equació, es proposen diversos mètodes de diferències finites, incloent mètodes explícits, implícits i ADE (Alternating Direction Explicit). Es proposen així mateix noves modificacions del mètode de Newton per a la solució dels sistemes no lineals derivats. En la secció 3.2 s'aplica per primera vegada el mètode front-fixing a un problema de valoració d'opcions amb canvi de règims. Atés que en aquest model hi ha diversos règims, apareixen unes quantes fronteres lliures pel que la transformació de variables porta a un sistema d'equacions no lineals. Este sistema es resol amb un esquema en diferències finites explícit. Per mitjà de l'enfocament de Von Neumann es prova l'estabilitat de l'esquema.

El 4t capítol oferix una nova tècnica per a la resolució de problemes de valoració d'opcions Americanes basada en la racionalitat dels inversors. Apareix una funció de la intensitat que es pot reduir en el cas més simple a la tècnica de penalització (penal method). Este enfocament té en compte el possible comportament irracional dels inversors. En la secció 4.2 s'aplica esta tècnica al model de canvi de règims el que porta a un nou model que té en compte el possible exercici irracional, així com diversos estats del mercat. L'enfocament del paràmetre de racionalitat junt amb una transformació logarítmica permeten construir un esquema numèric eficient sense aplicar el mètode front-fixing o la coneguda formulació de LCP (Linear Complementarity Problem). Es proposa una família d'esquemes ponderats tant per a opcions americanes de tipus "vanilla" com per al model de canvi de règims. S'estudien les propietats qualitatives de la funció d'intensitat $\mathrm{i}$ de les solucions numèriques.

El 5é capítol es dedica a la valoració d'opcions d'actius múltiples. Una transformació apropiada permet l'eliminació del terme de derivades 
mixtes evitant inconvenients computacionals i possibles problemes d' estabilitat.

Les conclusions es mostren al 6é capítol. Es posa en relleu diversos aspectes de la present tesi. Tots els models considerats i els mètodes numèrics van acompanyats de diversos exemples i simulacions. S'estudia la convergència numèrica que confirma l'estudi teòric de la consistència. Les condicions d'estabilitat són corroborades amb exemples numèrics. Els resultats es comparen amb mètodes rellevants de la bibliografia mostrant l'eficiència dels mètodes proposats. 



\begin{abstract}
The present $\mathrm{PhD}$ thesis is focused on numerical analysis and computing of finite difference schemes for several relevant option pricing models that generalize the Black-Scholes model. A careful analysis of desirable properties for the numerical solutions of option pricing models as the positivity, stability and consistency, is provided.

In order to handle the free boundary that arises in American option pricing problems, various transformation techniques based on front-fixing method are applied and studied. Special attention is paid to multi-asset option pricing, such as exchange or spread option. An appropriate transformation allows eliminating of the cross derivative term. Transformation techniques of partial differential equations to remove convection and reaction terms are studied in order to simplify the models and avoid possible troubles of stability.

This thesis consists of six chapters. The first chapter is an introduction containing definitions of option and related terms and derivation of the Black-Scholes equation as well as general aspects of theory of finite difference schemes, including preliminaries on numerical analysis.

Chapter 2 is devoted to solve linear Black-Scholes model for American put and call options. A Landau transformation and a new frontfixing transformation are applied to the free boundary value problem. It leads to a non-linear partial differential equation (PDE) in a fixed domain. Stable and consistent explicit numerical schemes are proposed preserving positivity and monotonicity of the solution in accordance with the behaviour of the exact solution.

Efficiency of the front-fixing method demonstrated in Chapter 2 has motivated us to apply the method to some more complicated nonlinear models. A new change of variables resulting in a time dependent boundary instead of fixed one, is applied to nonlinear Black-Scholes
\end{abstract}


model for American options, such as Barles and Soner and Risk Adjusted Pricing models, an effective numerical algorithm is constructed for a general case of non-constant volatility in Section 3.1. In order to solve the resulting equation various finite difference methods are constructed, including explicit, implicit and alternating direction explicit methods. Studying well-known Newton's method for solving nonlinear system allows to propose new modifications of the method. In Section 3.2 the front-fixing method is tested by American option pricing problem with regime switching model. Since in this model there are several regimes, i.e. several optimal stopping boundaries, the frontfixing transformation leads to a system of nonlinear equations that is solved by using explicit finite difference scheme. The stability of the proposed explicit FDM is studied basing on Von Neumann's approach.

Chapter 4 provides a new alternative approach for solving American option pricing problem based on rationality of investor. There exists an intensity function that can be reduced in the simplest case to penalty approach. The model takes into account possible irrational behaviour of the investor. This approach is applied to regime switching model resulting new model that takes into account possible irrational exercise as well as several states of market in Section 4.2. The rationality parameter approach together with a logarithmic transformation allows to construct effective numerical scheme without applying front-fixing method or LCP formulation. For both, vanilla American option and regime switching model, a family of weighted schemes is constructed. Qualitative properties of the intensity function and numerical solutions are studied.

Chapter 5 deals with multi-asset option pricing. Appropriate transformation allows eliminating of the cross derivative term avoiding computational drawbacks and possible troubles of stability.

Concluding remarks are given in Chapter 6. All the considered models and numerical methods are accompanied by several examples and simulations. The convergence rate is computed confirming the theoretical study of consistency. Stability conditions are tested by numerical examples. Results are compared with known relevant methods in the literature showing efficiency of the proposed methods. 


\section{Acknowledgements}

There are several people who have directly or indirectly contributed to this thesis, and here I would like to acknowledge them.

Foremost, I would like to express my sincere thanks to my supervisors, Professor Dr. Rafael Company Rossi and Professor Dr. Lucas Antonio Jódar Sánchez from the Institute of Multidisciplinary Mathematics in Polytechnic University of Valencia for providing me this opportunity. Their guidance has been, at every stage of the research, a constant source of motivation and knowledge. Their clear and objective explanations on various aspects of the numerical analysis helped me in my research. I could not have asked for better mentors.

My sincere gratitude to Professor Dr. Choi-Hong Lai from University of Greenwich and Professor Dr. Carlos Vázquez Cendón from University of A Coruña for their hospitality during my secondments, for our fruitful discussions and collaboration.

I would like to thank all members of the ITN-STRIKE: Novel Methods in Computational Finance for this opportunity and a lot of trainings and interesting events. It is a pleasure for me to be a part of such team. Special thanks to Shih-Hau Tan, Álvaro Leitao y Zuzana Bučková for all their help.

My sincere thanks to colleagues at the Institute of Multidisciplinary Mathematics in Polytechnic University of Valencia. Special thanks to Toni Vidal for improving my Spanish and for the beautiful Valencia translation of the abstract.

I would like to thank my parents, sister and brother for their constant support and encouragement. Most of all I would like to thank my husband Anton, whose constant patience, love, affection and motivation were of immense help in the successful completion of this thesis. Words are not enough to express my sincere appreciation for all my family. 



\section{Contents}

List of Figures

List of Tables

List of Publications

1 Introduction 1

1.1 Black-Scholes model ................. 3

1.2 General aspects of finite difference methods . . . . . . . . 6

1.2.1 Introduction to theory of grids . . . . . . . . . 6

1.2.2 Finite difference approximation of differential operators . 7

1.2.3 Statement of finite difference problem . . . . . . . . . 10

1.2.4 Some aspects of numerical analysis ......... 11

2 Linear Black-Scholes model for American options 15

2.1 Front-fixing method for American put option with no dividends . . 17

2.1.1 Qualitative properties of the scheme . . . . . . . . 20

2.1.2 Numerical examples .............. 25 25

2.2 Front-fixing method for American call option . . . . . . . . . 29

2.2.1 Qualitative properties of the scheme . . . . . . . . . 31

2.2.2 Numerical examples . . . . . . . . . . . . . 38

2.3 New efficient front-fixing method for American option pricing . . 43

2.3.1 Qualitative properties of the scheme . . . . . . . . . 46

2.3.2 Numerical examples .............. 51 51 
3 Front-fixing method for some advanced models

3.1 Nonlinear Black-Scholes models . . . . . . . . . . . . . . 59

3.1.1 Moving domain transformation . . . . . . . . 5 59

3.1.2 Preliminary computational algorithms . . . . . . . 62

3.1 .3 Explicit Schemes . . . . . . . . . . . . . . 64 64

3.1.4 Implicit numerical methods . . . . . . . . . . 66

3.1.5 Numerical examples . . . . . . . . . . . . . . . 71

3.2 Regime switching model . . . . . . . . . . . . . 78

3.2.1 Multi-variable fixed domain transformation . . . . . . . 79

3.2.2 Discretization and numerical schemes construction . . . 81

3.2.3 Von Neumann stability analysis . . . . . . . . . . 86

3.2.4 Local truncation error and consistency . . . . . . . . 88

3.2.5 Numerical examples . . . . . . . . . . . . . . 89

4 Behavioural modelling of option pricing 97

4.1 Pricing of American option with rationality parameter . . . . . . . 101

4.1.1 Numerical solution with irrational exercise . . . . . . . . 103

4.1.2 Transformation and explicit finite difference method . . . 106

4.1.3 Properties of intensity function and solution . . . . . . . . 107

4.1.4 Stability and consistency . . . . . . . . . . . . . . 112

4.1.5 Numerical examples . . . . . . . . . . . . . . 113

4.2 Regime switching model with rationality parameter . . . . . . . . 121

4.2.1 Weighted finite difference scheme for PDE problem . . . 122

4.2.2 Qualitative properties of the scheme . . . . . . . . . 124

4.2.3 Numerical examples . . . . . . . . . . . . 132

5 Valuation of multi-asset options 137

5.1 Exchange options . . . . . . . . . . . . . . . . 139

5.2 Spread options . . . . . . . . . . . . . . . . . 141

5.2.1 Removing the cross-derivative term . . . . . . . . . 142

5.2.2 Numerical analysis of the method . . . . . . . . . . 146

5.2.3 American spread options . . . . . . . . . . . . . . 149

5.2.4 Numerical examples . . . . . . . . . . . . 150

6 Conclusions 155

References 


\section{List of Figures}

2.1 Stable (left) and unstable (right) solution by the proposed frontfixing method depending on mesh ratio $\mu \ldots \ldots$. . . . . 26

2.2 Analytical ("analytic") and computed by the proposed method ("method") values of optimal stopping boundaries in Example 2.2.2 . . . . 40

2.3 The difference between computed value and estimation of $c_{2}^{n}$ for the problem with the parameters 2.90) and step sizes $h=10^{-3}$ and $k=6.25 \cdot 10^{-6} \ldots \ldots \ldots \ldots \ldots \ldots \ldots$. . . . . . . . . . .

2.4 Optimal stopping boundary in Example 2.3.2 by using proposed transformation and fixed-domain transformation [109]. . . . . . 53

2.5 Optimal stopping boundary in Example 2.3.3 computed by explicit and implicit methods. . . . . . . . . . . . . 54 54

2.6 The function $c(x, \tau)$ calculated by the proposed fully implicit method. 55

3.1 Moving grid. . . . . . . . . . . . . . . . . . . 64

3.2 A comparison of the free boundary $S_{f}(\tau)$ for RAPM model for various risk premium measures $R=5,15,40,70,100$ with the corresponding free boundary for $R=0$ (bold line). . . . . . . 74

3.3 A comparison of the free boundary $S_{f}(\tau)$ for Barles and Soner's model for $a=0,0.01,0.07,0.13 \ldots \ldots \ldots \ldots$

3.4 Difference between solutions by explicit method and iterative explicit method with $h_{0}=10^{-2}$ and various $k \ldots \ldots \ldots$

3.5 Stable numerical solution with $k=10^{-4}$. . . . . . . . . 76

3.6 Unstable numerical solution with $k=2.6 \cdot 10^{-3} . \ldots \ldots$

3.7 American put option price curves at $\tau=T$ and its payoff. . . . . . 90

3.8 Optimal stopping boundary for regime 1 and regime 2 (stability condition is fulfilled) . . . . . . . . . . . . . 91 


\section{LIST OF FIGURES}

3.9 Optimal stopping boundary for regime 1 and regime 2 (stability condition is broken). . . . . . . . . . . . 91

3.10 Delta of option with parameters 3.78 in both regimes. . . . . . 94

3.11 Gamma of option with parameters $\sqrt{3.78}$ in both regimes. . . . . . 94

3.12 American put option price curves at $\tau=T$ for the four regime model and its payoff. . . . . . . . . . . . 95

3.13 Optimal stopping boundary for the four regime American put option with parameters $(3.80) \ldots \ldots \ldots \ldots$

4.1 Numerical solution the intensity function belonging to family 4.4 with $\lambda=100$ for various values of $\theta \ldots \ldots \ldots \ldots$

4.2 Numerical solution the intensity function belonging to family 4.5 with $\lambda=100$ for various values of $\theta \ldots \ldots \ldots \ldots$

4.3 Numerical solution for the intensity function belonging to family (4.5) for various values of $\lambda \ldots \ldots \ldots \ldots \ldots$

4.4 Option price with $\lambda=1 \ldots \ldots \ldots \ldots$. . . . . . . . . . . . .

4.5 Option price with $\lambda=1000 \ldots \ldots \ldots \ldots$

4.6 Oscillations of the solution of the problem with parameters 4.36 and $h=10^{-2}, k=10^{-2} \ldots \ldots \ldots \ldots \ldots \ldots \ldots$

4.7 Stable numerical solution of the problem with parameters 4.36 with $h=10^{-2}, k=10^{-4} \ldots \ldots \ldots \ldots \ldots \ldots$

4.8 Numerical solution of the problem with parameters 4.79 . . . . . 134

4.9 Numerical solution of the problem with parameters 4.79) with matrix $(4.80) \ldots \ldots \ldots \ldots$

4.10 Numerical solution of the problem with parameters (4.79) with various $\lambda$ (Regime 1$) \ldots \ldots \ldots \ldots \ldots$

4.11 Numerical solution of the problem with parameters (4.79) with various $\lambda$ (Regime 2$) \ldots \ldots \ldots \ldots 135$

4.12 Stable and not stable solutions (Regime 1). . . . . . . . . . . 136

4.13 Stable and not stable solutions (Regime 2). . . . . . . . . . 136

5.1 Optimal exercise ratio in time: calculated by the proposed in Section 2.2 method (left) and presented in [86]. . . . . . . . . . 140

5.2 Numerical domain after removing cross derivative term transformation. . . . . . . . . . . . . . . . . . 144

5.3 European spread option (stable), condition (5.43) is fulfilled. . . . 151

5.4 European spread option price, condition $(5.43)$ is broken. . . . . . 151 


\section{List of Tables}

1.1 Finite difference approximation of the first derivative at the point $x_{j}$. 9

1.2 Finite difference approximation of the second derivative at the point $x_{j} \ldots \ldots \ldots \ldots \ldots \ldots \ldots \ldots$

2.1 American put option values obtained by the proposed method with various spatial step. . . . . . . . . . . . . . . 26

2.2 American put option values obtained by the proposed method with various mesh ratio $\mu \ldots \ldots \ldots \ldots . \ldots \ldots$

2.3 Comparison of various relevant methods. . . . . . . . . . 28

2.4 American call option values calculated by the proposed front-fixing method (FF) and other methods. . . . . . . . . . . . . 39

2.5 American call option values with parameters (2.90) calculated by various methods. . . . . . . . . . . . . . 4 40

2.6 Computational efficiency of various methods in Example 2.2.3. . . 42

2.7 Computational efficiency of various methods in Example 2.3.1. . . 52

2.8 Comparison of the proposed method with other methods for parameters (2.128) . . . . . . . . . . . . . . . . . . 53

2.9 Comparison of the computational time and accuracy for explicit and implicit methods. . . . . . . . . . . . . . 55

3.1 CPU-time (sec) of linear and binary search algorithms. . . . . . 63

3.2 RMSE with respect to CPU-time for different $h_{0}$ and fixed $k=$

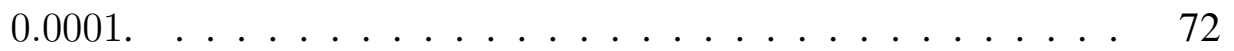

3.3 RMSE with respect to CPU-time for different $k$ and fixed $h_{0}=0.01$. 73

3.4 The maximum distance between the solution of the problem (3.12) by explicit and iterative explicit method. . . . . . . . . . . 74 


\section{LIST OF TABLES}

3.5 Spectral radius of matrix $J_{\text {approx }}^{-1} J$, where $J_{\text {approx }}$ is calculated by various methods. . . . . . . . . . . . . . 77

3.6 Comparison of American put option prices in a two regime model. 90

3.7 Comparison of explicit and implicit methods. Time step of explicit and implicit methods are denoted correspondingly by $k_{\text {expl }}$ and $k_{i m p l} .92$

3.8 RMSE and computational time for fixed $k=10^{-4}$ and various $h$. . 92

3.9 RMSE and computational time for fixed $h=10^{-2}$ and various $k$. . 93

3.10 Comparison of the efficiency of the IFV and proposed method (FF). 93

3.11 Option values at $S=10.0$ in Regime 1 for various mesh ratios $\mu=\frac{k}{h^{2}}$ and spatial step $h=10^{-2} \ldots \ldots \ldots \ldots \ldots$

3.12 Comparison of American put option prices in a four-regime model 96

4.1 Convergence to the true value with increasing $\lambda$ for family (4.4) for fixed $h=10^{-2}, k=10^{-4} \ldots \ldots \ldots \ldots \ldots$. . . . . . . . . . . .

4.2 Convergence to the true value with increasing $\lambda$ for family (4.5) for fixed $h=10^{-2}, k=10^{-4}$. . . . . . . . . . . . 115

4.3 Comparison of different methods for the American option with rational exercise (classical problem). . . . . . . . . . . . . 118

4.4 Comparison of the approximations with large rationality parameter $\left(\lambda=10^{4}\right)$ and a rational case reference approximation. . . . . . 118

4.5 Spatial and temporal convergence rates of explicit, implicit and Crank-Nicolson schemes for $\lambda=10^{4}$ and $\lambda=1 . \ldots \ldots$. . . 119

4.6 CPU-time in seconds of proposed methods for fixed $h=10^{-} 2$ and various $k \ldots \ldots \ldots \ldots \ldots \ldots \ldots$

4.7 Convergence of the solution for various intensity functions $f_{1}, f_{2}$, $f_{3}$ to American option price and comparison with front-fixing (FF) and Tree methods. The tests are done with explicit scheme $(\theta=0)$, $h=10^{-2}$ and time step $k=10^{-4} \ldots \ldots \ldots \ldots \ldots \ldots$

4.8 Convergence rate in space of the proposed $\theta$-scheme for $\lambda=10^{3}$. 135

4.9 Convergence rate in time of the proposed $\theta$-scheme for $\lambda=10^{3}$. . 136

5.1 European spread option price calculated by the proposed method (FDM) and Analytical approximation (5.44) . . . . . . . . . 152

5.2 American spread option price calculated by various methods for $E=0,2,4 \ldots \ldots \ldots \ldots \ldots \ldots$

5.3 CPU-Time in sec (first row) and Absolute difference (second row) for different methods depending on number of time-steps for fixed number of space steps for parameters $(5.45) \ldots \ldots \ldots$. . . . . 


\section{List of Publications}

\section{Published Papers}

1. R. Company, V. N. Egorova, and L. Jódar. Solving American option pricing models by the front fixing method: Numerical analysis and computing. Abstract and Applied Analysis, (Article ID 146745):9, 2014.

2. V.N. Egorova, S.-H. Tan, C.-H. Lai, R. Company, and L. Jódar. Moving boundary transformation for American call options with transaction cost: finite difference methods and computing. International Journal of Computer Mathematics, 2015:1-18, 2015. DOI:10.1080/00207160.2015.1108409 Published online: 08 Dec 2015.

3. R. Company, V.N. Egorova, and L. Jódar. Constructing positive reliable numerical solution for American call options: A new front-fixing approach. Journal of Computational and Applied Mathematics, 291:422 - 431, 2016.

4. V.N. Egorova, R. Company, and L. Jódar. A new efficient numerical method for solving American option under regime switching model. Computers \& Mathematics with Applications, 71(1):224 - 237, 2016.

5. R. Company, V.N. Egorova, L. Jódar, and C. Vázquez. Finite difference methods for pricing American put option with rationality parameter: numerical analysis and computing. Journal of Computational and Applied Mathematics, 304: 1-17, 2016.

6. R. Company, V.N. Egorova, L. Jódar, and C. Vázquez. Computing American option price under regime switching with rationality parameter. Journal of Computers \& Mathematics with Applications, 72: 741-754,2016. 


\section{Submitted Paper(s)}

- R. Company, V.N. Egorova, and L. Jódar. An efficient method for solving spread option pricing problem: numerical analysis and computing. submitted to Journal of Applied Mathematics and Computation, 2015.

\section{Chapter in Book(s)}

- V. Egorova , L. Jódar, R. Company. FDMs and transformation methods for nonlinear Black-Scholes equations, Online ECMI Newsletter 56, 2014, pp. 76-77.

- V. Egorova, R. Company and L. Jódar. Numerical solution of American option pricing models using front-fixing method, Chapter 30 in: Mathematical Modelling in Social Sciences and Engineering, Editors: J.C. Cortés, L. Jódar, R. Villanueva, Nova Publishers, 2014, pp. 311-319.

\section{Presentations in Conferences}

1. V.N. Egorova, R. Company, L. Jódar, "A Positive, stable and consistent frontfixing numerical scheme for American options", European Consortium for Mathematics in Industry (ECMI 2014) Conference, June 9-13, 2014, Taormina, Italy. Proceedings of the conference ISBN: 978-3-319-23412-0, in press.

2. V.N. Egorova, R. Company, L. Jódar, "Transforming American call option problem preserving qualitative properties of solution", Mathematical Modelling in Engineering \& Human Behaviour 2014 Conference, September 2-5, 2014, Instituto de Matemática Multidisciplinar-UPV, Valencia, Spain. Proceedings of the conference ISBN 978-84-606-5746-0, pp. 38-42.

3. V.N. Egorova, R. Company, L. Jódar, "Constructing positive reliable numerical solution for American options: a new front-fixing approach", Mathematical Methods in Economics and Industry, September 7-12, 2015, Bratislava, Slovakia.

4. V.N. Egorova, S.H. Tan, C. H. Lai, R. Company, L. Jódar, "New fixingdomain transformations for non-linear option pricing models", 6th Workshop Nonlinear PDEs and Financial Mathematics, 23-27 March, 2015, Zittau, Germany. 
5. V.N. Egorova, R. Company, L. Jódar, "Computing American options with regime switching model using front-fixing transformation", Stochastics \& Computational Finance 2015 - From Academia to Industry, July 6-10, 2015, Lisbon, Portugal.

6. V.N. Egorova, R. Company, L. Jódar, "Front-fixing transformation for regime switching model of American options", Mathematical Modelling in Engineering \& Human Behaviour 2015 Conference, September 9-11, 2015, Instituto de Matemática Multidisciplinar-UPV, Valencia, Spain. Proceedings of the conference ISBN 978-84-608-5355-8, pp. 129-134.

7. V.N. Egorova, R. Company, L.Jódar, C. Vázquez, "Finite difference methods for pricing American put option with rationality parameter", International Conference on Computational Finance, December 14-18, 2015, Greenwich, UK. Proceedings of the conference ISBN 978-19-065-6002-7, p. 38.

All the publications and the presentations have been partially supported by the European Union in the FP7- PEOPLE-2012-ITN program under Grant Agreement Number 304617 (FP7 Marie Curie Action, Project Multi-ITN STRIKE-Novel Methods in Computational Finance) and the Ministerio de Economía y Competitividad Spanish grant MTM2013-41765-P. 

The beginning is the most important part of the work.

Plato

CHAPTER

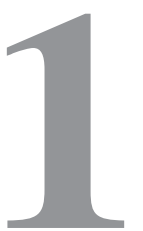

\section{Introduction}

American options are contracts allowing the holder the right to sell (buy) an asset at a certain price at any time until a pre-specified future date. They are widely traded in today's financial markets as its early exercise privilege. Therefore the pricing of American options plays an important role both in theory and in real derivative markets. The American option pricing problem can be posed either as a linear complementarity problem (LCP) or a free boundary value problem. These two different formulations have led to different methods for solving American options.

The optimal exercise boundary of an American option is not known a-priori and has to be determined as a part of the solution. Since the boundary of the domain of an American option is a free boundary, the valuation problem constitutes a free boundary value problem. In order to eliminate the explicit dependence on the free boundary the Black-Scholes equation can be reformulated as a linear complimentary problem (LCP), see [11], [35].

In our work we follow the free boundary value approach. The problem of finding optimal exercise boundary can be treated analytically or numerically. With respect to the analytical approach, Geske and Johnson [52] obtained a valuation formula for American puts expressed in terms of a series of compound-option functions (see also Barone-Adesi and Whaley [7] and Ju [65]). Analytical approaches to approximating the exact solution yield formulae which are difficult to use in 


\section{INTRODUCTION}

practice. It explains the considerable interest of numerical methods among the researchers and the wide range of numerical proposals in the recent literature.

Numerical methods were initiated by Brennan and Schwartz [16] and the convergence of their finite difference method was proved by Jaillet, Lamberton and Lapeyere [62]. Other relevant works using finite difference methods are Hull and White [59], Duffy [37], Wilmott et al. [111], Forsyth and Vetzal [48], Tavella and Randall [104], Tangman et al. [103].

A front-fixing method for free boundary value problem has been proposed by Crank [33]. Idea of the method is to transform the problem into a new non-linear partial differential equation where the free boundary appears as a new unknown function involved in the PDE problem. Wu and Kwok [112] applied the frontfixing technique to the field of option pricing. The front-fixing method is studied in other relevant papers (see [90], [109], [115].

We apply various transformations based on front-fixing method to American option pricing problem. However, the linear Black-Scholes model [10] provides an easy computable pricing formula of the option in an idealistic market with not realistic assumptions [14], [82]. This has motivated the development of new nonlinear models to take account of various realistic trading environment, such as transaction costs, illiquid market effects, etc. Therefore, various advanced models, such as Barles and Soner model[6], risk adjusted pricing methodology (RAPM) proposed by Kratka [75] and studied by Jandačka Ševčovič in [63], regime switching models are considered as well as behavioural modelling of option pricing and multiasset options.

Dealing with prices it is important to obtain not only fast solution, but guaranteed positivity. Therefore, apart from computational efficiency, the qualitative properties of the method are welcome to be studied. We provide numerical analysis of the proposed methods, including study of stability and consistency.

The numerical solution for every considered model is found. The numerical results confirms theoretical studies of stability and qualitative properties. Computational convergence rate is found for every proposed scheme. Results and compared with known results in the literature to show the potential advantages. The implementation of the schemes has been done by using MatLAB R2015a on processor Pentium(R) Dual-Core CPU E5700 3.00 GHz. 


\subsection{Black-Scholes model}

Option is a contract between buyer and holder that gives to its holder right to buy or sell underlying asset by fixed price called strike or exercise price [58], [111]. There are two types of options - call and put. Call option gives the right to buy the asset, while holder of put option may sell the asset by fixed price at the fixed moment. The process of buying or selling underlying asset is called exercise of option. Time of option existence is called maturity. At the moment of signing the option the holder has to pay to buyer certain amount of money that is called option value or option price. That is the key problem for many researches.

There are widely traded two main styles of options - European and American. The main difference between them is that European option has fixed exercise moment - maturity date, while American option may be exercised at any time up to the maturity. Therefore, price of American option has to be higher than European one on the same asset.

From the mathematical point of view option price is considered as a function on underlying asset and time as variables and several parameters:

- Strike price. Relation between asset price and strike price defines status of the option ("in the money", "out of the money") and option price.

- Time to maturity. Time works against the holder since the price of the option "out of the money" is decreasing as time tends to maturity date. It is called time decay. Bigger time to maturity means higher uncertainty.

- Volatility. Value of the option is proportional expected price instability of underlying asset.

- Dividend yield. Dividends reduce call option price and enhance value of put option, since these payments reduce asset price.

Classic Black-Scholes model requires several assumptions [58]:

- Dividends are not paid while option exists. Many companies pay dividends, so this assumption is not realistic. The easiest way to correct model is to discount asset price on amount of dividends. In the case of American call options absence of dividends makes holder to exercise the option at the maturity date. It means that American style proceeds to a European. 
- Market is efficient. It is supposed that market moves due to continuous Itó process. In other words, price fluctuations are expected and predicted by investors and trending stocks.

- Trading without transaction costs. This assumption is not realistic, because usually traders and investors pay additional cost that influence on the model.

- Fixed interest rate. In the real market this rate could change during holding option, through this the model gives wrong estimation.

- In Black-Scholes model, it is assumed that the probability distribution of the stock price is lognormal and the instantaneous log return is a geometric Brownian motion. However the market for the options shows that the geometric Brownian model for the underlying asset leads to underprice or overprice for these options [18].

The model was presented in 1973 and since that time many researches have improved the model by elimination described assumptions. In recent researches more complicated (usually nonlinear) models are considered. It allows to calculate the option price that fits (or is approximating) to the real market data.

Taking assumptions of the Black-Scholes model, we suppose that put option prices are impacted negatively by increasing interest rates. that is differentiable by $t$ and continuously differentiable by $S$. Using Itô formula, one gets

$$
d V=\sigma S \frac{\partial V}{\partial S} d S+\left(\mu S \frac{\partial V}{\partial S}+\frac{\sigma^{2}}{2} S^{2} \frac{\partial^{2} V}{\partial S^{2}}+\frac{\partial V}{\partial t}\right) d t
$$

where $\sigma$ is the volatility and $\mu$ is the risk-free rate.

Let us consider portfolio of just one option and $-\Delta$ number of stocks that is not defined. Then a portfolio value $\Pi$ can be found by the following expression

$$
\Pi=V-\Delta S
$$

Since $\Delta=$ const in a short amount of time $d t$, the change of portfolio value is

$$
d \Pi=d V-\Delta d S
$$

Thus, from (1.1) one gets

$$
d \Pi=\sigma S\left(\frac{\partial V}{\partial S}-\Delta\right) d S+\left(\mu S \frac{\partial V}{\partial S}+\frac{\sigma^{2}}{2} S^{2} \frac{\partial^{2} V}{\partial S^{2}}+\frac{\partial V}{\partial t}-\mu \Delta S\right) d t
$$


Value of $\Delta$ can be chosen as $\Delta=\frac{\partial V}{\partial S}$, such that

$$
d \Pi=\left(\frac{\partial V}{\partial t}+\frac{\sigma^{2}}{2} s^{2} \frac{\partial^{2} V}{\partial S^{2}}\right) d t .
$$

Earnings of portfolio $\Pi$ is defined by value $r \Pi d t$ for period $d t$. If the right hand side of (1.2) is greater than this amount, then the holder can get risk-free profit by investing value of $\Pi$ to the portfolio. In this case there appear an arbitrage possibility. From the other hand, if the right hand side of $(1.2)$ is smaller than $r \Pi d t$, then the holder can invest the money to bank assets. It also leads to risk-free profit, in other words, arbitrage possibility. Since Black-Scholes model means no arbitrage, the only possible situation is the equality [58],

$$
r \Pi d t=\left(\frac{\partial V}{\partial t}+\frac{\sigma^{2}}{2} s^{2} \frac{\partial^{2} V}{\partial S^{2}}\right) d t,
$$

that leads to the Black-Scholes equation after some calculations

$$
\frac{\partial V}{\partial t}+\frac{\sigma^{2}}{2} S^{2} \frac{\partial^{2} V}{\partial S^{2}}+r S \frac{\partial V}{\partial S}-r V=0 .
$$

In order to simplify the notation, function $V(S, t)$ is usually denoted by $c(S, t)$ for call and $p(S, t)$ for put. Corresponding capital letters are used for American style options.

Terminal and boundary conditions have to be defined as well. The option price is known at the maturity $(t=T)$, thus the terminal condition is defined by the payoff function, that for European and American options is

$$
p(S, T)=(E-S)^{+}, \quad c(S, T)=(S-E)^{+} .
$$

Boundary conditions can be obtained from the natural behaviour of option. Thus, for European option one boundary ( $S \rightarrow \infty$ for call and $S=0$ for put) is discounted strike price, while the rest boundary condition is $V=0$. The boundary conditions for American options will be established in the following section.

Option pricing problem leads to partial differential equation (PDE) of the second order. In the case of American options, one deals with free boundary value problem. One of the most effective methods for solving such problems is so-called finite difference method (FDM). It allows to get approximate solution of the PDE by using a system of algebraic equations. Theoretical background of the method is presented in the following section. 


\subsection{General aspects of finite difference methods}

This section is devoted to recall ideas of finite difference methods for partial differential equations of the second order and theoretical aspects related to the method.

\subsubsection{Introduction to theory of grids}

In order to use finite difference method for a given problem the following two steps have to be done:

1. Substitute continuous region for discrete computational domain. If the original region is infinite, it has to be truncated in a such way that boundary conditions holds true.

2. Substitute the differential operator for a finite difference operator and establish a discrete analogue of initial and boundary conditions.

As the result of these actions a system of algebraic equation is obtained. The numerical solution of the system is an approximate solution of the original PDE problem. It is clear that numerical solution could not be found at every point of the continuous region, therefore it is logic to choose set of points called grid from the region and compute solution only at these points called nodes. If distance between any two neighbour nodes is constant the grid is called uniform, otherwise - nonuniform. The computational grid of $M+1$ space points and $N+1$ time levels on domain $\left[x_{\min }, x_{\max }\right] \times[0, T]$ with respective step sizes $h$ and $k$

$$
h=\frac{x_{\max }-x_{\min }}{M}, \quad k=\frac{T}{N},
$$

is the set of points $\left(x_{j}, \tau^{n}\right)$, where

$$
x_{j}=x_{\text {min }}+h j, \quad j=0, . ., M, \quad \tau^{n}=k n, \quad n=0, . ., N .
$$

Function defined at the nodes is called grid function. Supposing that function $P(x, \tau)$ of continuous arguments is an element of some functional space $H_{0}$ is exact solution, the approximate solution at the node $\left(x_{j}, \tau^{n}\right)$ is denoted by $p_{j}^{n} \approx P(x, \tau)$, $p(x, \tau) \in H_{h, k}$ is a grid function, where $H_{h, k}$ is a space of grid functions. The main question of theory of numerical methods is how far the approximate solution from the exact solution is. How to estimate it if grid function and continuous function are from the different spaces $H_{h, k}$ and $H_{0}$ ? One way is to supply the grid function 
by interpolation, for example, at the rest points of continuous region. As the result continuous function $\tilde{p}(x, \tau) \in H_{0}$ is obtained. Proximity of function is defined by a norm in space of continuous functions $\|\cdot\|_{0}$. Another way is to translate function $P(x, \tau)$ to the grid. As the result, grid function $\tilde{P}(x, \tau) \in H_{h, k}$ is obtained. Then the difference $(\tilde{P}-p) \in H_{h, k}$ is a grid function. Closeness of $p$ to $\tilde{P}$ is defined by a norm $\|\cdot\|_{h, k}$. It is logical to require that (see [98])

$$
\lim _{h \rightarrow 0, k \rightarrow 0}\|u\|_{h, k}=\|U\|_{0}, \quad \forall U \in H_{0}
$$

\subsubsection{Finite difference approximation of differential operators}

Let $L$ be a differential operator on function $v=v(x)$. Substitution of derivatives for finite differences gives a linear combination $L_{h} v_{h}$ on some set of grid nodes $\Omega(x)$, called stencil. Then finite difference approximation $L_{h} v_{h}$ of differential operator $L v$ takes the following form

$$
\left(L_{h} v_{h}(x)\right)_{i}=\sum_{x_{j} \in \Omega\left(x_{i}\right)} A_{h}\left(x_{i}, x_{j}\right) v_{h}\left(x_{j}\right),
$$

where $A_{h}\left(x_{i}, x_{j}\right)$ are the coefficients, $h$ is a step size.

Finite difference approximation is usually studied at the fixed point $x_{i}$. First of all, the stencil has to be chosen for the approximation. Then the coefficients have to be defined to approximate the differential operator. These coefficients can be found from Taylor's expansion of function $v$.

For example, the first derivative can be approximated by using current node $x$ and one neighbour node $x \pm h$. We start with Taylor's expansion at this point:

$$
v(x \pm h)=v(x) \pm h v^{\prime}(x)+\frac{h^{2}}{2} v^{\prime \prime}(x)+O\left(h^{3}\right) .
$$

Then from (1.4) $v^{\prime}(x)$ is derived as follows

$$
v^{\prime}(x)=\frac{v(x+h)-v(x)}{h}-\frac{h}{2} v^{\prime \prime}(x)+O\left(h^{2}\right),
$$

or

$$
v^{\prime}(x)=\frac{v(x)-v(x-h)}{h}+\frac{h}{2} v^{\prime \prime}(x)+O\left(h^{2}\right) .
$$

Besides, from (1.4),

$$
v^{\prime}(x)=\frac{v(x+h)-v(x-h)}{2 h}+O\left(h^{2}\right) .
$$


Therefore, the first derivative can be approximated by the following expressions:

$$
\begin{aligned}
v_{x}^{+} & =\frac{v(x+h)-v(x)}{h}, \\
v_{x}^{-} & =\frac{v(x)-v(x-h)}{h}, \\
v_{x}^{0} & =\frac{v(x+h)-v(x-h)}{2 h} .
\end{aligned}
$$

Expression $v_{x}^{+}$is called forward difference, $v_{x}^{-}$- backward and $v_{x}^{0}$ - central differences.

Definition 1.2.1 (Truncation error). Truncation error (Error of approximation) $\phi$ is the error made by truncating an infinite sum and approximating it by a finite sum (see [3], p. 20). In other words, $\phi$ is calculated as a difference between the approximated value and the exact value.

Often, truncation error also includes discretization error, which is the error that arises from taking a finite number of steps in a computation to approximate an infinite process. For example, in numerical methods for ordinary differential equations, the continuously varying function that is the solution of the differential equation is approximated by a process that progresses step by step, and the error that this entails is a discretization or truncation error. From (1.5)-(1.7) one gets

$$
\phi=v_{x}^{+}-v^{\prime}(x)=O(h), \phi=v_{x}^{-}-v^{\prime}(x)=O(h), \phi=v_{x}^{0}-v^{\prime}(x)=O\left(h^{2}\right) .
$$

Definition 1.2.2 (Order of approximation). With the previous notation, $L_{h}$ approximates differential operator $L$ with order $m>0$ around the point $x$, if

$$
\phi(x)=L_{h} v(x)-L v(x)=O\left(h^{m}\right) .
$$

Thus, forward and backward differences are the first-order approximation, while the central difference is of the second order.

In regard to the second derivatives, analogous procedure allows to obtain the second-order approximation:

$$
v^{\prime \prime}(x)=\frac{v(x+h)-2 v(x)+v(x-h)}{2 h}-\frac{h^{2}}{12} v^{(4)}(x)+O\left(h^{3}\right) .
$$




\begin{tabular}{|c|c|}
\hline Approximation & Truncation error \\
\hline$\frac{1}{h}\left(v_{j+1}-v_{j}\right)$ & $O(h)$ \\
\hline$\frac{1}{h}\left(v_{j}-v_{j-1}\right)$ & $O(h)$ \\
\hline$\frac{1}{2 h}\left(v_{j+1}-v_{j-1}\right)$ & $O\left(h^{2}\right)$ \\
\hline$\frac{1}{2 h}\left(-3 v_{j}+4 v_{j+1}-v_{j+2}\right)$ & $O\left(h^{2}\right)$ \\
\hline$\frac{1}{2 h}\left(3 v_{j}-4 v_{j-1}+v_{j-2}\right)$ & $O\left(h^{2}\right)$ \\
\hline$\frac{1}{12 h}\left(v_{j-2}-8 v_{j-1}+8 v_{j+1}-v_{j+2}\right)$ & $O\left(h^{4}\right)$ \\
\hline
\end{tabular}

Table 1.1: Finite difference approximation of the first derivative at the point $x_{j}$.

\begin{tabular}{|c|c|}
\hline Approximation & Truncation error \\
\hline$\frac{1}{h^{2}}\left(v_{j+2}-2 v_{j+1}+v_{j}\right)$ & $O(h)$ \\
\hline$\frac{1}{h}\left(v_{j}-2 v_{j-1}+v_{j-2}\right)$ & $O(h)$ \\
\hline$\frac{1}{h^{2}}\left(v_{j+1}-2 v_{j}+v_{j-1}\right)$ & $O\left(h^{2}\right)$ \\
\hline$\frac{1}{h^{2}}\left(-v_{j+3}+4 v_{j+2}-5 v_{j+1}+2 v_{j}\right)$ & $O\left(h^{2}\right)$ \\
\hline$\frac{1}{h^{2}}\left(-v_{j-3}+4 v_{j-2}-5 v_{j-1}+2 v_{j}\right)$ & $O\left(h^{2}\right)$ \\
\hline$\frac{1}{12 h^{2}}\left(-v_{j-2}+16 v_{j-1}-30 v_{j}+16 v_{j+1}-v_{j+2}\right)$ & $O\left(h^{4}\right)$ \\
\hline
\end{tabular}

Table 1.2: Finite difference approximation of the second derivative at the point $x_{j}$.

Following this idea many finite difference approximations can be defined. Central differences are mostly used because of the order of approximation. However, at the boundary of the computational domain one-sided differences could be useful. In Tables 1.1 and 1.2 some common difference approximations of the first and second derivatives correspondingly are presented.

The finite difference method is used not only for ordinary differential equations (ODEs), but for partial differential equations (PDEs). In that case each partial differential operator is approximated by one of the formulas in Tables 1.1, 1.2.

Example 1.2.1. Let us consider a homogeneous heat equation

$$
\frac{\partial u}{\partial t}=\frac{\partial^{2} u}{\partial x^{2}}
$$

Let us denote approximate solution at the node $\left(x_{j}, t^{n}\right)$ by $v_{j}^{n}$. Uniform grid is considered with time step $k$ and spatial step $h$. We choose forward difference in time 
and central difference for spatial derivative. Then the finite difference equation takes the following form

$$
\frac{v_{j}^{n+1}-v_{j}^{n}}{k}=\frac{v_{j-1}^{n}-2 v_{j}^{n}+v_{j+1}^{n}}{h^{2}} .
$$

In (1.13) four-points stencil is used. Due to Tables 1.1 and 1.2 the approximation is of the first order in time and of the second order in space. Usually it is written as $O(k)+O\left(h^{2}\right)$.

Note that grid is not necessary uniform. However, analogous procedure of the approximation based on Taylor's expansion is used. If the three-point stencil with nodes $x_{j-1}=x-h_{-}, x_{j}=x$ and $x_{j+1}=x+h_{+}$is chosen, $h_{-} \neq h_{+}$. Then central difference approximation of the first derivative takes the following form

$$
v^{\prime}(x)=\frac{v_{j+1}-v_{j-1}}{h_{+}+h_{-}}+O\left(h_{-}-h_{+}\right) .
$$

Thus central difference on non-uniform stencil is of the first order [98].

The finite difference approximations of differential operators have been considered. However, the real problem in fields of physics or finance contains also initial and boundary conditions that allows to find unique solution. Therefore, let us consider statement of finite difference problem.

\subsubsection{Statement of finite difference problem}

Definition 1.2.3 (Finite difference scheme). A set of finite difference equations that approximate given differential equation and additional boundary and initial conditions is called finite difference scheme.

The procedure of the scheme construction is considered in the following example.

Example 1.2.2. The heat equation is considered:

$$
\begin{aligned}
L u & =\frac{\partial u}{\partial t}-\frac{\partial^{2} u}{\partial x^{2}}=f(x, t), \quad 0<x<1,0<t \leq T, \\
u(0, t) & =\mu_{1}(t), \quad u(1, t)=\mu_{2}(t), \\
u(x, 0) & =u_{0}(x) .
\end{aligned}
$$




\subsection{General aspects of finite difference methods}

Here $(1.15)$ are boundary conditions and $(1.16)$ is initial condition.

The uniform grid is chosen:

$$
x_{j}=j h, j=0, \ldots, M, \quad t^{n}=n k, k=0, \ldots, N .
$$

Denoting $v_{j}^{n}=u\left(x_{j}, t^{n}\right)$ and $\phi_{j}^{n}=f\left(x_{j}, t^{n}\right)$, the scheme on four-point stencil takes the following form:

$$
\begin{gathered}
\frac{v_{j}^{n+1}-v_{j}^{n}}{k}=\frac{v_{j+1}^{n}-2 v_{j}^{n}+v_{j-1}^{n}}{h^{2}}+\phi_{j}^{n}, \quad 1 \leq j \leq M-1,0 \leq n<N, \\
v_{0}^{n}=\mu_{1}\left(t^{n}\right), \quad v_{M}^{n}=\mu_{2}\left(t^{n}\right), \quad 0 \leq n \leq N, \\
v_{j}^{0}=u_{0}\left(x_{j}\right), \quad 0 \leq j \leq M .
\end{gathered}
$$

Scheme (1.17)-1.19) is called explicit: values at the next $n+1$-th time level are found by using values from the previous $n$-th time level. Implicit scheme uses the following finite difference equation

$$
\frac{v_{j}^{n+1}-v_{j}^{n}}{k}=\frac{v_{j+1}^{n+1}-2 v_{j}^{n+1}+v_{j-1}^{n+1}}{h^{2}}+\phi_{j}^{n}, \quad 1 \leq j \leq M-1,0 \leq n<N .
$$

Thus, the system with three-diagonal matrix has to be solve to obtain values $v_{j}^{n+1}$. For solving such a system Thomas algorithm is usually used [32], [98].

If PDE is nonlinear, then implicit scheme leads to nonlinear system that is usually solved by iterative algorithm, for example, Newton's or quasi-Newton's methods [78].

Constructing numerical method for a given problem one has to take into account that the solution is approximate. Therefore, the keynote of theoretical study of FDM is qualitative properties of the scheme, including stability and consistency. These properties give the preliminary information about how accurate solution is and what step sizes can be chosen to improve the properties of the scheme. In the following section definitions that are used in the present work are given.

\subsubsection{Some aspects of numerical analysis}

For FDM theory is typical the assumption that solution of the PDE exists and has a required number of derivatives [101], [102].

Since numerical solution is approximate, the difference between exact solution and numerical one is called truncation error. It can be calculated by several formulas. In the present work the Root Mean Square Error (RMSE) is used: 


$$
R M S E_{h}=\sqrt{\frac{1}{n} \sum_{j=1}^{n}\left(v_{j}^{*}-v_{j}\right)^{2}},
$$

where $v_{j}^{*}=u\left(x_{j}\right)$ is exact solution at the point $x_{j}$ and $v_{j}$ is approximated solution obtained by FDM with step size $h$.

Further RMSE is used for convergence rate calculation.

Definition 1.2.4 (Convergence). A finite difference scheme $F(v)$ approximating PDE Lu is a convergent scheme if for any $x$ and $t$, as $(j h, n k)$ tends to $(x, t)$, numerical solution $v$ converges to exact solution $u$ as step sizes $h$ and $k$ tend to zero.

In order to understand how fast the numerical solution converges to the exact solution, convergence rate $\gamma$ for each coordinate $x$ or $t$ is calculated as follows

$$
\gamma_{h}=\frac{\ln R M S E_{h}-\ln R M S E_{h / 2}}{\ln 2} .
$$

However, very often exact solution is not known. In that case one can suppose that numerical solution obtained on refined grid (with very small step sizes) is exact or another expression of convergence rate is used

$$
\gamma_{h}=\log _{2} \frac{\left\|v_{h / 2}-v_{h}\right\|}{\left\|v_{h / 4}-v_{h / 2}\right\|},
$$

where $v_{h}$ is the numerical solution obtained by using FDM with step size $h$ and $\|\cdot\|$ is one the vector norms, for example, infinity-norm $\|\cdot\|_{\infty}$ (the maximum of the absolute values of the components).

Related to convergence concept is consistency.

Definition 1.2.5 (Consistency). Under consistency of a numerical scheme with respect to a partial differential equation we understand that the exact solution of the PDE approximates well the exact theoretical solution of the finite difference scheme as the step size discretization tends to zero.

Mostly, study of consistency leads to calculation of the order of approximation of the scheme.

Constructing numerical scheme it is important to guarantee that oscillations do not occur and that some perturbation in initial data does not destroy the scheme. All these phenomenons lead to understanding of stability. For the sake of clarity and 


\subsection{General aspects of finite difference methods}

since there are many criteria for stability in the literature, let us recall the following definition that we follow in present work (see for instance [76], p. 92).

Definition 1.2.6 (Stability). The numerical scheme is said to be $\|\cdot\|_{\infty}$-stable in the computational domain if for every partition with $k$ and $h$,

$$
\max _{n}\left\|v^{n}\right\|_{\infty} \leq C\left\|v^{0}\right\|_{\infty}
$$

where $v^{n}$ is the approximate solution at the moment $t^{n}=n k$ and $C$ is independent of $h, k$ constant.

This definition allows that solution grows, but the growth is bounded by some constant $C$. If a numerical scheme is stable for any partition $h$ and $k$ without any restriction, it is called unconditionally stable.

Sometimes study of stability by the definition is hard work and there are another methods that allow to find stability condition (of find that the scheme is unconditionally stable). One of the well-known techniques is von Neumann stability analysis [101], [102]. The idea of the method is to express initial values in terms of finite Fourier series and then consider growth of the function. This method will be considered below. 



\section{Linear Black-Scholes model for American options}

The main advantage of American options is the possibility of early exercise. This fact in the economical sense makes American options more attractive for investors. From the mathematical point of view this possibility of early exercise leads to a free boundary problem, where $S_{f}(t)$ is the switching point that is called $o p$ timal stopping boundary. The optimal strategy of the investor depends on value of $S_{f}(t)$ at current moment: for $0<S<S_{f}(t)$ the put option should be exercised and the call option should be hold. For $S>S_{f}(t)$ vice-a-versa: the put option should be hold and the call option should be exercised. In further work the holding region is considered, because option price in exercise region is defined by the payoff function.

The American put option price $P(S, \tau)$, where $\tau=T-t$ is the time to maturity, with constant dividend yield $q$, is the solution of linear partial differential equation of the second order

$$
\frac{\partial P}{\partial \tau}=\frac{1}{2} \sigma^{2} S^{2} \frac{\partial^{2} P}{\partial S^{2}}+(r-q) S \frac{\partial P}{\partial S}-r P, \quad S>S_{f}(\tau), \quad 0<\tau \leq T,
$$

supplied with the following initial conditions 


$$
P(S, 0)=\max (E-S, 0), \quad S_{f}(0)=E \max \left(\frac{r}{q}, 1\right),
$$

and the boundary conditions

$$
P\left(S_{f}(\tau), \tau\right)=E-S_{f}(\tau), \quad \lim _{S \rightarrow \infty} P(S, \tau)=0 .
$$

Since an additional unknown function $S_{f}(\tau)$ is included in the free boundary formulation, one extra condition is necessary. This condition is called smooth pasting condition and requires that the slope of the option price curve at the free boundary coincides with the slope of payoff function. Thus, for put option it is presented as follows

$$
\frac{\partial P}{\partial S}\left(S_{f}(\tau), \tau\right)=-1
$$

As it is explained in previous section, dividend payments influence on option price and investor's strategy such that put option becomes more expensive and price of call option has to be discounted. In other words, if there is no dividend payment $(q=0)$, then the optimal strategy for holder of American call is to exercise the option at the maturity ( see [111], chapter 7.7, [59]). In that case the American call becomes European one. Because of that the problem for American call option is considered just for $q>0$ [59].

American call option price model is given by [111] as the free boundary PDE

$$
\frac{\partial C}{\partial \tau}=\frac{1}{2} \sigma^{2} S^{2} \frac{\partial^{2} C}{\partial S^{2}}+(r-q) S \frac{\partial C}{\partial S}-r C, \quad 0 \leq S<S_{f}(\tau), \quad 0<\tau \leq T
$$

together with the boundary and initial conditions

$$
\begin{array}{r}
C(S, 0)=\max (S-E, 0), \quad S_{f}(\tau)=E \max \left(\frac{r}{q}, 1\right) \\
C\left(S_{f}(\tau), \tau\right)=S_{f}(\tau)-E, \quad \frac{\partial C}{\partial S}\left(S_{f}(\tau), \tau\right)=1 \quad C(0, \tau)=0 .
\end{array}
$$

Analytical or closed form solution of the free boundary problems 2.1)- 2.4 and (2.5)-(2.7) does not exist. Therefore numerical methods are employed for solving. In this chapter various front-fixing transformations with finite difference methods are used to construct effective and stable numerical solution. Special attention is paid to study positivity and monotonicity of the numerical solution as well as stability and consistency of the proposed schemes. 


\subsection{Front-fixing method for American put option with no dividends}

First of all, classical Black-Scholes model for American put option 2.1)-(2.4) without dividend payments $(q=0)$ is considered. A dimensionless Landau transformation [79] is proposed as follows

$$
x=\ln \frac{S}{S_{f}(\tau)}, \quad p(x, \tau)=\frac{P(S, \tau)}{E}, \quad s_{f}(\tau)=\frac{S_{f}(\tau)}{E} .
$$

The spatial variable $x$ transfers the free boundary domain $S>S_{f}(\tau)$ to the fixed, but unbounded domain $(0 ; \infty)$. In new coordinates $(x, \tau)$ the problem $(2.1)$ (2.4) is rewritten in the following normalized form

$$
\frac{\partial p}{\partial \tau}=\frac{1}{2} \sigma^{2} \frac{\partial^{2} p}{\partial x^{2}}+\left(r-\frac{\sigma^{2}}{2}\right) \frac{\partial p}{\partial x}-r p+\frac{s_{f}^{\prime}}{s_{f}} \frac{\partial p}{\partial x}, \quad x>0, \quad 0<\tau \leq T
$$

where $s_{f}^{\prime}$ denotes the derivative of $s_{f}$ with respect to $\tau$. The new transformed equation (2.9) is a nonlinear PDE on the domain $(0, \infty) \times(0, T]$ since $s_{f}$ and its derivative are involved. Using transformation $(2.8)$ the initial and boundary conditions for the original problem (2.1)-(2.4) have to be rewritten as follows

$$
\begin{gathered}
p(x, 0)=0, \quad x \geq 0, \quad s_{f}(0)=1, \\
\frac{\partial p}{\partial x}(0, \tau)=-s_{f}(\tau), \quad p(0, \tau)=1-s_{f}(\tau), \\
\lim _{x \rightarrow \infty} p(x, \tau)=0 .
\end{gathered}
$$

In order to solve numerically problem (2.9)-(2.12), computational domain has to be truncated. Let us introduce $x_{\max }$ large enough to translate the boundary condition 2.12, i.e. $p\left(x_{\max }, \tau\right)=0$. Then the problem 2.9 -2.12 can be studied on the fixed domain $\left[0, x_{\max }\right] \times(0, T]$. The value $x_{\max }$ is chosen following the criterion pointed out in [66].

Let us introduce the computational grid of $M+1$ spatial nodes and $N+1$ time levels with respective step sizes $h$ and $k$ :

$$
\begin{gathered}
h=\frac{x_{\max }}{M}, \quad k=\frac{T}{N}, \\
x_{j}=h j, \quad j=0, \ldots, M, \quad \tau^{n}=k n, \quad n=0, \ldots, N .
\end{gathered}
$$


The approximate value of $p(x, \tau)$ at the point $x_{j}$ and time $\tau^{n}$ is denoted by $p_{j}^{n} \approx p\left(x_{j}, \tau^{n}\right)$. Then a forward two time-level and centred in space scheme is constructed for internal spacial nodes $1 \leq j \leq M-1, \quad 0 \leq n \leq N-1$ as follows:

$$
\begin{aligned}
\frac{p_{j}^{n+1}-p_{j}^{n}}{k} & =\frac{\sigma^{2}}{2} \frac{p_{j-1}^{n}-2 p_{j}^{n}+p_{j+1}^{n}}{h^{2}} \\
& +\left(\frac{2 r-\sigma^{2}}{2}+\frac{s_{f}^{n+1}-s_{f}^{n}}{k s_{f}^{n}}\right) \frac{p_{j+1}^{n}-p_{j-1}^{n}}{2 h}-r p_{j}^{n}
\end{aligned}
$$

Parabolic mesh ratio is denoted by $\mu=\frac{k}{h^{2}}$, then the scheme 2.15 takes the following form

$$
p_{j}^{n+1}=\tilde{a}_{1} p_{j-1}^{n}+a_{2} p_{j}^{n}+\tilde{a}_{3} p_{j+1}^{n}
$$

where

$$
\begin{gathered}
\tilde{a}_{1}^{n}=a_{1}-\frac{s_{f}^{n+1}-s_{f}^{n}}{2 h s_{f}^{n}}, \quad \tilde{a}_{3}^{n}=a_{3}+\frac{s_{f}^{n+1}-s_{f}^{n}}{2 h s_{f}^{n}}, \\
a_{1,3}=\frac{\mu}{2}\left(\sigma^{2} \mp\left(r-\frac{\sigma^{2}}{2}\right) h\right), \quad a_{2}=1-\sigma^{2} \mu-r k .
\end{gathered}
$$

The boundary conditions (2.11) and (2.12) are discretised as follows

$$
\frac{p_{1}^{n}-p_{-1}^{n}}{2 h}=-s_{f}^{n} ; \quad p_{0}^{n}=1-s_{f}^{n},
$$

where $x_{-1}=-h$ is an auxiliary point out of the domain. By considering the equation (2.9) at the point $x_{0}=0, \tau>0$, what involves the assumption of the existence of $\frac{\partial^{2} p}{\partial x^{2}}(0, \tau)$ and replacing of the boundary conditions 2.11) into equation 2.9) at $(0, \tau)$ a new boundary condition takes the following form (see [112],[115])

$$
\frac{1}{2} \sigma^{2} \frac{\partial^{2} p}{\partial x^{2}}(0, \tau)+\frac{\sigma^{2}}{2} s_{f}(\tau)-r=0
$$

and its central difference discretization

$$
\frac{\sigma^{2}}{2} \frac{p_{1}^{n}-2 p_{0}^{n}+p_{-1}^{n}}{h^{2}}+\frac{\sigma^{2}}{2} s_{f}^{n}-r=0
$$

From $\sqrt{2.19}$ and $\sqrt{2.21}$ the value of $p_{-1}^{n}$ can be eliminated obtaining the relationship

$$
p_{1}^{n}=\alpha-\beta s_{f}^{n}, \quad n \geq 1,
$$

between the free boundary approximation $s_{f}^{n}$ and $p_{1}^{n}$, where 


$$
\alpha=1+\frac{r h^{2}}{\sigma^{2}}, \quad \beta=1+h+\frac{1}{2} h^{2} .
$$

By using the scheme (2.15) for $j=1$ and evaluating (2.22) for $n+1$ time level, the free boundary $s_{f}^{n+1}$ can be expressed as

$$
s_{f}^{n+1}=d^{n} s_{f}^{n}, \quad 0 \leq n \leq N-1,
$$

where

$$
d^{n}=\frac{\alpha-\left(a_{1} p_{0}^{n}+a_{2} p_{1}^{n}+a_{3} p_{2}^{n}-\frac{p_{2}^{n}-p_{0}^{n}}{2 h}\right)}{\frac{p_{2}^{n}-p_{0}^{n}}{2 h}+\beta s_{f}^{n}} .
$$

After expression 2.24 the value $s_{f}^{n+1}$ can be replaced in 2.15, 2.22 and 2.19 to obtain values $p_{j}^{n+1}, \quad 0 \leq j \leq M-1$. Then the numerical scheme for the problem (2.9) - 2.12) can be rewritten for any $n=0, \ldots, N-1$, in the following algorithmic form

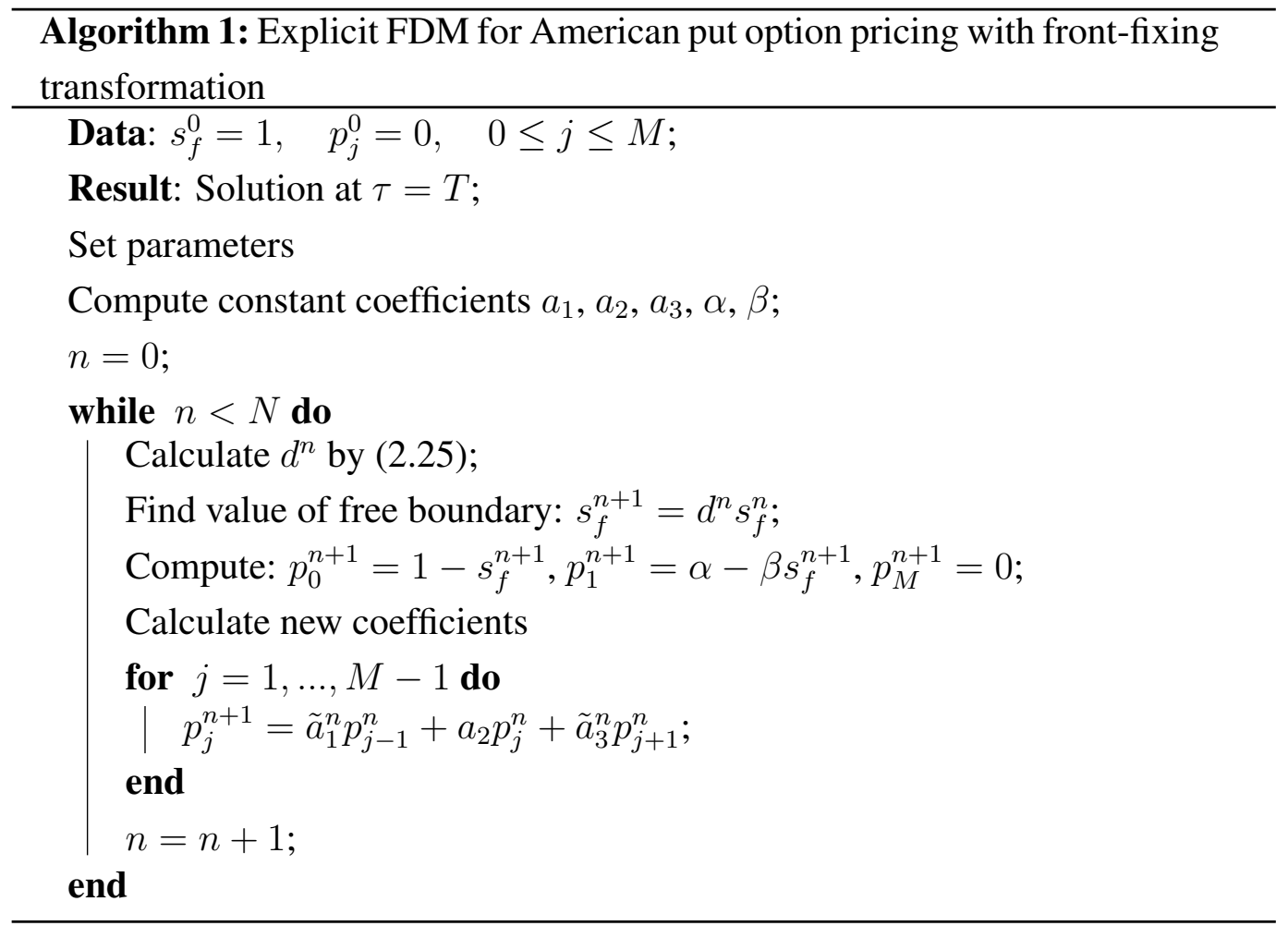




\subsubsection{Qualitative properties of the scheme}

In this section the free boundary non-increasing monotonicity as well as the positivity and non-increasing spacial monotonicity of the numerical option price are shown.

Lemma 2.1.1. Assuming that the step sizes $h$ and $k$ satisfy the conditions:

$$
\begin{array}{ll}
C 1: & h \leq \frac{\sigma^{2}}{\left|r-\frac{\sigma^{2}}{2}\right|}, \quad r \neq \frac{\sigma^{2}}{2}, \\
C 2: & k \leq \frac{h^{2}}{\sigma^{2}+r h^{2}},
\end{array}
$$

then the coefficients (2.18) are non-negative. If $r=\frac{\sigma^{2}}{2}$, then under the condition $C 2$, the coefficients are non-negative.

Proof. From 2.18) for non-negativity of $a_{1}$ it is necessary that

$$
\sigma^{2}-\left(r-\frac{\sigma^{2}}{2}\right) h \geq 0
$$

If $r \leq \frac{\sigma^{2}}{2}$ from 2.18 note that $a \geq 0$ for any $h>0$. Otherwise 2.26 is satisfied under the condition $C 1$. From (2.18) $a_{2}$ is non-negative under the condition $C 2$. If $r \geq \frac{\sigma^{2}}{2}$ non-negativity of $a_{3}$ is guaranteed by 2.18 for any $h>0$. Otherwise $a_{3}$ is non-negative under the condition $C 1$.

The following lemma prepares the study of positivity of the numerical solution as well as the monotonicity of the free boundary sequence $s_{f}^{n}$, that will be established in a further result.

Lemma 2.1.2. Let $\left\{p_{j}^{n}, s_{f}^{n}\right\}$ be the numerical solution of scheme (2.15)-(2.12) for the transformed American put option problem (2.9) and let $d^{n}$ be defined by (2.25). Then under hypothesis of the Lemma 2.1.1, for small enough $h$, one verifies

1. For each fixed $n$

$$
0<d^{n} \leq 1
$$

2. Values $p_{j}^{n+1} \geq 0$ for $j=0, \ldots, M ; n=0, . . N-1$.

3. $p_{j}^{n+1} \geq p_{j+1}^{n+1}$ for $j=0, \ldots M-1 ; n=0, . . N-1$. 
Proof. The induction principle is used for proof. From initial conditions $p_{j}^{0}=0$, $0 \leq j \leq M$, and therefore $p_{j}^{0} \geq p_{j+1}^{0}$. From 2.23, 2.25) and hypothesis $C 1$ of Lemma 2.1.1 one gets

$$
0<d^{0}=\frac{\alpha}{\beta} \leq 1
$$

Since $s_{f}^{0}=1$, from boundary conditions $2.11-2.12$ and 2.28 one gets

$$
0<s_{f}^{1}=d^{0} \leq 1 ; \quad p_{0}^{1}=1-d^{0} \geq 0 ; \quad p_{1}^{1}=0 .
$$

Furthermore, from $2.16 p_{j}^{1}=0$ for $j=2, . . M$.

For the sake of clarity let us show firstly that $0<d^{1} \leq 1$. From 2.25, 2.23 and 2.29) it follows that

$$
d^{1}=1-a_{1} \frac{\beta-\alpha}{\alpha \beta-\frac{\beta-\alpha}{2 h}}=1-a_{1} \frac{h+h^{2}\left(\frac{1}{2}-\frac{r}{\sigma^{2}}\right)}{\frac{1}{2}+h\left(\frac{3}{4}+\frac{r}{2 \sigma^{2}}+O\left(h^{2}\right)\right)} .
$$

Under condition $C 1$ in Lemma 2.1.1 $a_{1}>0$, for small enough values of $h$ one gets

$$
0<d^{1} \leq 1
$$

Let us assume the induction hypothesis that conclusions hold true for index $n-1$, that is

$$
0<d^{n-1} \leq 1, \quad p_{j}^{n} \geq 0, \quad p_{j}^{n} \geq p_{j+1}^{n},
$$

and prove that conclusions hold true for index $n$. By denoting

$$
\begin{aligned}
f^{n} & =1+\frac{r h^{2}}{\sigma^{2}}-\left(a_{1} p_{0}^{n}+a_{2} p_{1}^{n}+a_{3} p_{2}^{n}-\frac{p_{2}^{n}-p_{0}^{n}}{2 h}\right) \\
g^{n} & =\frac{p_{2}^{n}-p_{0}^{n}}{2 h}+\left(\frac{1}{2}\left(1+(1+h)^{2}\right)\right) s_{f}^{n}
\end{aligned}
$$

$d^{n}$ takes the following form

$$
d^{n}=\frac{f^{n}}{g^{n}}
$$

For $n>2$, using Taylor's expansion, one gets the approximation of the involved derivatives and boundary conditions

$$
p_{2}^{n}=1+\frac{4 r h^{2}}{\sigma^{2}}-\left(1+2 h+2 h^{2}\right) s_{f}^{n}+O\left(h^{3}\right) .
$$




\section{LINEAR BLACK-SCHOLES MODEL FOR AMERICAN OPTIONS}

From 2.33) and 2.31) numerator $f^{n}$ takes the form

$$
f^{n}=r h\left[k+\frac{r k h+2(1-r k)}{\sigma^{2}}\right]+\left(\frac{h^{2}}{2}(1-r k)-k h \frac{\sigma^{2}}{2}\right) s_{f}^{n}+O\left(h^{2}\right),
$$

and verifies $f^{n}>0$ since $k<\frac{h}{r h+\sigma^{2}}$ under condition $C 2$ of Lemma 2.1.1 and for $h<1$.

From 2.33) and 2.25) denominator $g^{n}$ is positive for small enough values of $h$, since

$$
g^{n}=\frac{p_{2}^{n}-p_{0}^{n}}{2 h}+\left(1+h+\frac{h^{2}}{2}\right) s_{f}^{n}=\frac{2 r h}{\sigma^{2}}+\frac{h^{2}}{2} s_{f}^{n}+O\left(h^{2}\right)>0 .
$$

From 2.32 and previous comments one gets $d^{n}>0$. In order to prove that $d^{n} \leq 1$ let us consider the difference $f^{n}-g^{n}$. By using (2.34) and (2.35) under hypothesis of Lemma 2.1 .2 one can obtain

$$
f^{n}-g^{n}=k h\left(r \frac{\sigma^{2}-2 r+r h}{\sigma^{2}}-\frac{r h+\sigma^{2}}{2} s_{f}^{n}\right)+O\left(h^{2}\right) .
$$

Note that if $\sigma^{2}<2 r$ then 2.36 is non-positive for small enough values of $h$. However, even if $\sigma^{2} \geq 2 r$, the Samuelson asymptotic limit [99] $s_{f}^{n} \geq \frac{2 r}{2 r+\sigma^{2}}$ (see [112], p. 87) guarantees the non-positivity of (2.36). Therefore $d^{n} \leq 1$.

In order to prove the positivity of the solution $\left\{p_{j}^{n+1}\right\}$ the non-negativity of coefficients $\tilde{a}_{1}^{n}$ and $\tilde{a}_{3}^{n}$ appearing in equation $(2.16)$ is a sufficient condition. From 2.17) $\tilde{a}_{1}^{n}$ is positive since

$$
\tilde{a}_{1}^{n}=a_{1}-\frac{s_{f}^{n+1}-s_{f}^{n}}{2 h s_{f}^{n}}=a_{1}-\frac{d^{n}-1}{2 h} \geq a_{1} \geq 0 .
$$

From 2.18 and 2.35) the sign of $\tilde{a}_{3}^{n}$ is the same as the sign of $\left(2 h a_{3} g^{n}+f^{n}-g^{n}\right)$ and from (2.18), 2.34), 2.35) and $s_{f}^{n} \leq 1$, one gets for small enough values of $h$

$$
2 h a_{3} g^{n}+f^{n}-g^{n}>r k+\left(\sigma^{2} \mu+r k\right) \frac{r h^{2}}{\sigma^{2}}-\frac{k h^{2} \sigma^{2}}{4}>0 .
$$

Under hypothesises of induction (2.30) together with positivity of coefficients $\tilde{a}^{n}$ and $\tilde{c}^{n}$ the positivity of $\left\{p_{j}^{n+1}\right\}$ is proved. Moreover, $\left\{p_{j}^{n+1}\right\}$ is non-increasing with respect to index $j$ from 2.16 , since

$$
p_{j}^{n+1}-p_{j+1}^{n+1}=\tilde{a}_{1}^{n}\left(p_{j-1}^{n}-p_{j}^{n}\right)+a_{2}\left(p_{j}^{n}-p_{j+1}^{n}\right)+\tilde{a}_{3}^{n}\left(p_{j+1}^{n}-p_{j+2}^{n}\right) \geq 0 .
$$


Summarizing the following result has been established:

Theorem 2.1.1. Under assumptions of Lemma 2.1.2 the numerical scheme (2.16) for solving the American option transformed problem guarantees the following properties of the numerical solution:

- Non-increasing monotonicity and positivity of values $s_{f}^{n}, \quad n=0, \ldots, N$;

- Positivity of the vectors $p^{n}, \quad n=0, \ldots, N$;

- Non-increasing monotonicity of the vectors $p^{n}=\left(p_{0}^{n}, \ldots p_{M}^{n}\right)$ with respect to space indexes for each fixed $n=0, \ldots, N$.

Proof. The monotonicity and positivity of the values $s_{f}^{n}$ follow from the condition (2.27). Since initial conditions are trivial, coefficients of the scheme (2.16) are positive, the values of $p_{j}^{n}$ are also positive. Monotonicity of the vectors $p^{n}$ is the third statement of the Lemma 2.1.2 and is proved by (2.37).

Theorem 2.1.2. Under assumptions of Lemma 2.1.2 the numerical scheme (2.16)(2.19) for solving transformed problem (2.9)-(2.12) is $\|\cdot\|_{\infty}$-stable.

Proof. Since for each fixed $n,\left\{p_{j}^{n}\right\}$ is a non-increasing sequence with respect to $j$, then according to the boundary condition $(2.19)$ and positivity of $s_{f}^{n}$ since $(2.27)$, one gets

$$
\left\|P^{n}\right\|_{\infty}=p_{0}^{n}=1-s_{f}^{n}<1, \quad 0 \leq n \leq N .
$$

Thus, the scheme is $\|\cdot\|_{\infty}$-stable.

With the respect to consistency, let us write the numerical scheme 2.16$)$ in the form

$$
\begin{aligned}
F\left(p_{j}^{n}, s_{f}^{n}\right) & =\frac{p_{j}^{n+1}-p_{j}^{n}}{k}-\frac{1}{2} \sigma^{2} \frac{p_{j-1}^{n}-2 p_{j}^{n}+p_{j+1}^{n}}{h^{2}} \\
& -\left(r-\frac{\sigma^{2}}{2}\right) \frac{p_{j+1}^{n}-p_{j-1}^{n}}{2 h}+r p_{j}^{n}-\frac{s_{f}^{n+1}-s_{f}^{n}}{k s_{f}^{n}} \frac{p_{j+1}^{n}-p_{j-1}^{n}}{2 h}=0 .
\end{aligned}
$$

Let us denote by $\tilde{p}_{j}^{n}=p\left(x_{j}, \tau^{n}\right)$ the exact theoretical solution value of the PDE at the mesh point $\left(x_{j}, \tau^{n}\right)$, and let $\tilde{S}_{f}^{n}=s_{f}\left(\tau^{n}\right)$ be the exact solution of the free boundary at time $\tau^{n}$. The scheme $(2.38)$ is said to be consistent with 


$$
L\left(p, s_{f}\right)=\frac{\partial p}{\partial \tau}-\frac{1}{2} \sigma^{2} \frac{\partial^{2} p}{\partial x^{2}}-\left(r-\frac{\sigma^{2}}{2}\right) \frac{\partial p}{\partial x}+r p-\frac{s_{f}^{\prime}}{s_{f}} \frac{\partial p}{\partial x}=0,
$$

if the local truncation error

$$
T_{j}^{n}\left(\tilde{p}, \tilde{S}_{f}\right)=F\left(\tilde{p}_{j}^{n}, \tilde{S}_{f}^{n}\right)-L\left(\tilde{p}_{j}^{n}, \tilde{S}_{f}^{n}\right)
$$

satisfies

$$
T_{j}^{n}\left(\tilde{p}, \tilde{S}_{f}\right) \rightarrow 0, \quad \text { as } \quad h \rightarrow 0, \quad k \rightarrow 0 .
$$

Assuming the existence of the continuous partial derivatives up to order two in time and up to order four in space, using Taylor's expansion about $\left(x_{j}, \tau^{n}\right)$ one gets

$$
\begin{aligned}
T_{j}^{n}\left(\tilde{p}, \tilde{S}_{f}\right) & =k E_{j}^{n}(3)-\frac{\sigma^{2}}{2} h^{2} E_{j}^{n}(2)+\left(r-\frac{\sigma^{2}}{2}\right) h^{2} E_{j}^{n}(1) \\
& -k E_{j}^{n}(4) \frac{\partial p}{\partial x}\left(x_{j}, \tau^{n}\right)-h^{2} E_{j}^{n}(1) \frac{1}{\tilde{S}_{f}^{n}} \frac{d S_{f}}{d \tau}\left(\tau^{n}\right)-k h^{2} E_{j}^{n}(4) E_{j}^{n}(1),
\end{aligned}
$$

where

$$
\begin{aligned}
E_{j}^{n}(1) & =\frac{1}{6} \frac{\partial^{3} p}{\partial x^{3}}\left(x_{j}, \tau^{n}\right), & E_{j}^{n}(2) & =\frac{1}{12} \frac{\partial^{4} p}{\partial x^{4}}\left(x_{j}, \tau^{n}\right), \\
E_{j}^{n}(3) & =\frac{1}{2} \frac{\partial^{2} p}{\partial \tau^{2}}\left(x_{j}, \tau^{n}\right), & E_{j}^{n}(4) & =\frac{k}{2 \tilde{S}_{f}} \frac{d^{2} s_{f}}{d \tau^{2}}\left(\tau^{n}\right) .
\end{aligned}
$$

Equations 2.39) and 2.40) show the local truncation error of the numerical scheme 2.15) with respect to the PDE (2.9). In order to complete the consistency one has to rewrite the boundary conditions 2.11 , 2.12 in the following form

$$
\begin{aligned}
L_{1}\left(p, s_{f}\right) & =p(0, \tau)-1+s_{f}(\tau)=0, \\
L_{2}\left(p, s_{f}\right) & =\frac{\partial p}{\partial x}(0, \tau)+s_{f}(\tau)=0, \\
L_{3}\left(p, s_{f}\right) & =\frac{\sigma^{2}}{2} \frac{\partial^{2} p}{\partial x^{2}}(0, \tau)+\frac{\sigma^{2}}{2} s_{f}(\tau)-r=0 .
\end{aligned}
$$

Finite difference approximation for the boundary conditions takes the form

$$
\begin{aligned}
F_{1}\left(p, s_{f}\right) & =p_{0}^{n}-1+s_{f}^{n}=0, \\
F_{2}\left(p, s_{f}\right) & =\frac{p_{1}^{n}-p_{-1}^{n}}{2 h}+s_{f}^{n}=0, \\
F_{3}\left(p, s_{f}\right) & =\frac{\sigma^{2}}{2} \frac{p_{-1}^{n}-2 p_{0}^{n}+p_{1}^{n}}{h^{2}}+\frac{\sigma^{2}}{2} s_{f}^{n}-r=0 .
\end{aligned}
$$


Thus, truncation error can be estimated as follows

$$
\begin{aligned}
& T_{1}=F_{1}\left(p^{n}, s_{f}^{n}\right)-L_{1}\left(p^{n}, s_{f}^{n}\right)=0 \\
& T_{2}=F_{2}\left(p^{n}, s_{f}^{n}\right)-L_{2}\left(p^{n}, s_{f}^{n}\right)=O\left(h^{2}\right), \\
& T_{3}=F_{3}\left(p^{n}, s_{f}^{n}\right)-L_{3}\left(p^{n}, s_{f}^{n}\right)=O\left(h^{2}\right) .
\end{aligned}
$$

Boundary condition 2.41) is approximated exactly, without truncation error. Boundary conditions (2.42) and (2.43) are approximated with the second order, because we used central difference scheme for the first and the second derivatives. Summarized truncation error for the boundary condition

$$
T=T_{1}+T_{2}+T_{3}=O\left(h^{2}\right)
$$

The finite difference approximation is consistent with the boundary conditions with the second order. This result is formulated in the following theorem.

Theorem 2.1.3. The finite difference approximation is consistent with the equation (2.9) and boundary conditions, and local truncation error satisfies

$$
T_{j}^{n}(p)=O\left(h^{2}\right)+O(k)
$$

In the previous result we have assumed that the theoretical solution of the PDE [2.9 admits continuous partial derivatives up to certain order according to [101].

\subsubsection{Numerical examples}

In this section the results of the numerical experiments are presented to confirm the theoretical study. A comparison with other approaches is presented in this section.

Considered scheme is conditionally stable with constraints on space step and time step.

Example 2.1.1. The American put option pricing problem with the parameters as in 90$]$

$$
r=0.1, \quad \sigma=0.2, \quad T=1, \quad x_{\infty}=2,
$$

is considered. 


\section{LINEAR BLACK-SCHOLES MODEL FOR AMERICAN OPTIONS}
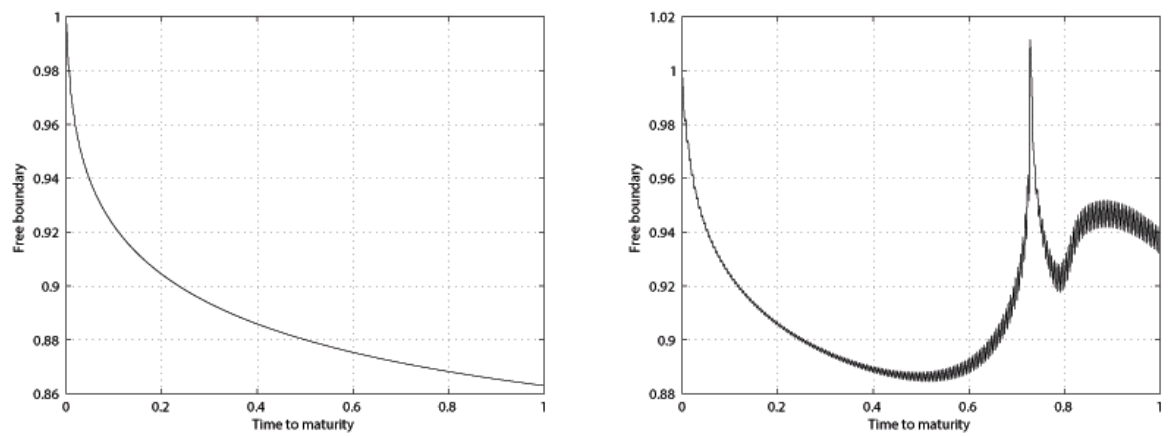

Figure 2.1: Stable (left) and unstable (right) solution by the proposed front-fixing method depending on mesh ratio $\mu$.

\begin{tabular}{|c|c|c|c|c|}
\hline Asset price & True value & $h$ & $h / 2$ & $h / 4$ \\
\hline 90 & 11.6974 & 11.4622 & 11.6870 & 11.6920 \\
100 & 6.9320 & 6.9719 & 6.9025 & 6.9229 \\
110 & 4.1550 & 4.0725 & 4.1429 & 4.1546 \\
120 & 2.5102 & 2.5623 & 2.5026 & 2.5101 \\
\hline
\end{tabular}

Table 2.1: American put option values obtained by the proposed method with various spatial step.

The spatial step is $h=0.01$ that satisfies condition $C 1$ of Lemma 2.1.1, and time step is variable. Dependence of stability on positivity of coefficient $a_{2}$ is demonstrated in Figure 2.1 (left: $\mu=24, a_{2}=1.0101$, right: $\mu=25.1, a_{2}=$ $-0.0043)$. Numerical tests show that the condition $C 2$ is critical for positivity of coefficient $a_{2}$ and, as a result, for the stability of the scheme.

It was theoretically proved that the order of approximation of the scheme is $O\left(h^{2}\right)+O(k)$. The results of the numerical experiments are presented in Table 2.1 For "True" values we use the "true values" from [96]. We consider the space steps: $h=2 \cdot 10^{-3}, h / 2=10^{-3} h / 4=5 \cdot 10^{-4}$ for fixed $\gamma=5$ to guarantee the stability.

The error is calculated for each case correspondingly,

$$
\epsilon_{1}=\sum\left(p_{\text {true }}-p_{h}\right)=0.0596, \quad \epsilon_{2}=0.015, \quad \epsilon_{3}=0.226
$$




\begin{tabular}{|c|c|c|c|c|}
\hline Asset price & True value & $\mu=5$ & $2 \mu$ & $4 \mu$ \\
\hline 90 & 11.6974 & 11.6870 & 11.6758 & 11.4563 \\
100 & 6.9320 & 6.9025 & 6.8856 & 6.9658 \\
110 & 4.1550 & 4.1429 & 4.1368 & 4.0668 \\
120 & 2.5102 & 2.5026 & 2.4786 & 2.5573 \\
\hline
\end{tabular}

Table 2.2: American put option values obtained by the proposed method with various mesh ratio $\mu$.

Thus, the order of approximation in space is

$$
\frac{\ln \frac{\epsilon_{1}}{\epsilon_{2}}}{\ln \frac{h}{h / 2}} \approx \frac{\ln \frac{\epsilon_{2}}{\epsilon_{3}}}{\ln 2} \approx \frac{\ln \frac{\epsilon_{1}}{\epsilon_{3}}}{\ln 4} \approx 2,
$$

The order of approximation in time is checked analogously with fixed space step $h=10^{-3}$. The results for different values of mesh ratio $\mu$ are presented in Table 2.2. The errors for corresponding values of $\mu$ are

$$
\epsilon_{4}=\sum\left(p_{\text {true }}-p_{\mu}\right)=0.0596, \quad \epsilon_{5}=0.1178, \quad \epsilon_{6}=0.2484,
$$

The order of approximation in time

$$
\frac{\ln \frac{\epsilon_{4}}{\epsilon_{5}}}{\ln 2} \approx \frac{\ln \frac{\epsilon_{5}}{\epsilon_{6}}}{\ln 2} \approx \frac{\ln \frac{\epsilon_{4}}{\epsilon_{6}}}{\ln 4} \approx 1
$$

coincides with the theoretical result.

In order to compare the proposed method with another known techniques, the front-fixing method with another transformation proposed in [90] is considered:

$$
x=\frac{S}{s_{f}(\tau)}, \quad p(x, \tau)=P(S, \tau)=P\left(x s_{f}(\tau), \tau\right) .
$$

Example 2.1.2. Let us compare front fixing method with another approach [91], based on the Mellin's transform. The parameters of the problem are $r=0.0488$, $\sigma=0.3, T=0.5833$.

To compare results of the explicit front-fixing method with the Mellin's transformation [91], we have to multiply our dimensionless value on $E=45$. Then for Mellin's transform method $s_{f}(T)=32.77$, while the proposed front-fixing method gives $s_{f}(T)=32.7655$. 


\begin{tabular}{|c|c|c|c|c|c|c|c|}
\hline S & True & MBM & HW & OCA & OS & PM & FF \\
\hline 90 & 11.6974 & 11.6889 & 11.6974 & 11.6975 & 11.6922 & 11.7207 & 11.6898 \\
100 & 6.9320 & 6.9203 & 6.9320 & 6.9321 & 6.9319 & 6.9573 & 6.9243 \\
110 & 4.1550 & 4.1427 & 4.1548 & 4.1550 & 4.1548 & 4.1760 & 4.1468 \\
120 & 2.5102 & 2.4996 & 2.5101 & 2.5102 & 2.5101 & 2.5259 & 2.5089 \\
\hline
\end{tabular}

Table 2.3: Comparison of various relevant methods.

Example 2.1.3. Let us consider set of parameters

$$
r=0.08, \quad \sigma=0.2, \quad T=3, \quad E=100 .
$$

In Table 2.3 the proposed explicit front-fixing method (FF) for American put is compared with another numerical methods shown in [96], such as

- The finite difference moving boundary method of Muthuraman (MBM) [89];

- Han-Wu algorithm (HW) transforms the Black-Scholes equation into a heat equation on an infinite domain [54];

- The optimal compact algorithm (OCA) for the heat equation [96];

- Ikonen and Toivanen [60] proposed an operator splitting technique (OS) for solving the linear complementarity problem.

- Penalty method (PM) is considered in [90] and [106].

One can see that the proposed method gives competitive results with guaranteed positivity and monotonicity of the solution and conditional stability of the scheme.

The results of this section have been published in [26]. 


\subsection{Front-fixing method for American call option}

In this chapter an explicit finite-difference scheme is proposed to solve the American call option pricing problem $(2.5)-(2.7)$.

Analogously to previous section 2.1 the dimensionless transformation for problem (2.5)- 2.7 )

$$
x=\ln \frac{B(\tau)}{S}, \quad c(x, \tau)=\frac{C(S, \tau)}{E}, \quad S_{f}(\tau)=\frac{B(\tau)}{E},
$$

is considered.

Under transformation 2.47) the problem 2.5 - 2.7) is rewritten in normalized form

$$
\frac{\partial c}{\partial \tau}=\frac{1}{2} \sigma^{2} \frac{\partial^{2} c}{\partial x^{2}}-\left(r-q-\frac{\sigma^{2}}{2}+\frac{S_{f}^{\prime}}{S_{f}}\right) \frac{\partial c}{\partial x}-r c, \quad x \geq 0,0<\tau \leq T,
$$

with new initial conditions

$$
S_{f}(0)=\max \left\{1, \frac{r}{q}\right\}, \quad c(x, 0)=\left\{\begin{array}{l}
0, \\
g(x), \quad r>q,
\end{array} \quad x \geq 0,\right.
$$

where

$$
g(x)=\max \left(\frac{r}{q} e^{-x}-1,0\right)
$$

and new transformed boundary conditions

$$
\begin{aligned}
\frac{\partial c}{\partial x}(0, \tau) & =-S_{f}(\tau), \\
c(0, \tau) & =S_{f}(\tau)-1, \\
\lim _{x \rightarrow \infty} c(x, \tau) & =0 .
\end{aligned}
$$

Following the ideas of [112] and in order to solve the numerical difficulties derived from the discretization at the numerical boundary, we assume that (2.48) holds true at $x=0$,

$$
\frac{\sigma^{2}}{2} \frac{\partial^{2} c}{\partial x^{2}}-\left(q+\frac{\sigma^{2}}{2}\right) S_{f}+r=0 .
$$

The equation $(2.48)$ is a nonlinear differential equation on the domain $[0, \infty) \times$ $(0, T]$. The problem $(2.48)-2.53)$ can be numerically solved on the fixed domain $\left[0, x_{\max }\right] \times(0, T]$. The value $x_{\max }$ is chosen following the criterion pointed out in [66]. 
As in the previous section, the uniform computational grid of $M+1$ space points and $N+1$ time levels with respective step sizes $h$ and $k$ is used (see (2.13)(2.14).

The approximate value of $c(x, \tau)$ at the point $x_{j}$ and time $\tau^{n}$ is denoted by $c_{j}^{n} \approx c\left(x_{j}, \tau^{n}\right)$, the approximate value of the free boundary is denoted by $S_{f}^{n} \approx$ $S_{f}\left(\tau^{n}\right)$. Then a forward two-time level and centred in a space explicit finite difference scheme is constructed for internal spacial nodes as follows

$$
\begin{aligned}
\frac{c_{j}^{n+1}-c_{j}^{n}}{k} & =\frac{1}{2} \sigma^{2} \frac{c_{j-1}^{n}-2 c_{j}^{n}+c_{j+1}^{n}}{h^{2}} \\
& -\left(r-q-\frac{\sigma^{2}}{2}+\frac{S_{f}^{n+1}-S_{f}^{n}}{k S_{f}^{n}}\right) \frac{c_{j+1}^{n}-c_{j-1}^{n}}{2 h}-r c_{j}^{n} .
\end{aligned}
$$

The equation (2.55) takes the following form

$$
c_{j}^{n+1}=a_{1}^{n} c_{j-1}^{n}+a_{2} c_{j}^{n}+a_{3}^{n} c_{j+1}^{n}, \quad 1 \leq j \leq M-1,
$$

where the coefficients $a_{1}^{n}$ and $a_{3}^{n}$ depend on the optimal exercise boundary $S_{f}^{n}$ and $S_{f}^{n+1}$ and $a_{i}, i=1,2,3$, are constant:

$$
\begin{gathered}
a_{1}^{n}=\frac{k}{2 h^{2}}\left(\sigma^{2}+\left(r-q-\frac{\sigma^{2}}{2}\right) h\right)+\frac{S_{f}^{n+1}-S_{f}^{n}}{2 h S_{f}^{n}}=a_{1}+\frac{S_{f}^{n+1}-S_{f}^{n}}{2 h S_{f}^{n}}, \\
a_{2}=1-\sigma^{2} \frac{k}{h^{2}}-r k \\
a_{3}^{n}=\frac{k}{2 h^{2}}\left(\sigma^{2}-\left(r-q-\frac{\sigma^{2}}{2}\right) h\right)-\frac{S_{f}^{n+1}-S_{f}^{n}}{2 h S_{f}^{n}}=a_{3}-\frac{S_{f}^{n+1}-S_{f}^{n}}{2 h S_{f}^{n}} .
\end{gathered}
$$

The central difference approximation of the first and second spatial derivative gives the following discrete expression of the boundary conditions 2.51, 2.52 and (2.54)

$$
\begin{gathered}
c_{0}^{n}=S_{f}^{n}-1, \quad \frac{c_{1}^{n}-c_{-1}^{n}}{2 h}=-S_{f}^{n}, \\
\frac{\sigma^{2}}{2} \frac{c_{-1}^{n}-2 c_{0}^{n}+c_{1}^{n}}{h^{2}}-\left(q+\frac{\sigma^{2}}{2}\right) S_{f}^{n}+r=0,
\end{gathered}
$$

where $c_{-1}^{n}$ means the value of the solution at the fictitious point $x=-h$, that should be eliminated later. 
The connection of the free boundary $S_{f}^{n}$ with option value $c_{1}^{n}$ at the same time level $n$ is presented as follows

$$
c_{1}^{n}=\alpha-\beta S_{f}^{n}, \quad n \geq 1
$$

where

$$
\alpha=-1-\frac{r h^{2}}{\sigma^{2}}, \quad \beta=-1+h-\left(\frac{q}{\sigma^{2}}+\frac{1}{2}\right) h^{2} .
$$

We use together 2.56) with $j=1$ and 2.60) to obtain the nonlinear law of the free boundary motion

$$
S_{f}^{n+1}=d^{n} S_{f}^{n}
$$

where

$$
d^{n}=\frac{a_{1} c_{0}^{n}+a_{2} c_{1}^{n}+a_{3} c_{2}^{n}+\frac{c_{2}^{n}-c_{0}^{n}}{2 h}-\alpha}{\frac{c_{2}^{n}-c_{0}^{n}}{2 h}-\beta S_{f}^{n}}
$$

In the following section qualitative scheme properties such as the free boundary non-decreasing monotonicity as well as the positivity and non-increasing spacial monotonicity of the numerical option price under transformation are proved analytically.

\subsubsection{Qualitative properties of the scheme}

Note, that from definition (2.57) the constant coefficients of the scheme $a_{1}, a_{2}$ and $a_{3}$ are positive for both cases: $r \leq q$ and $r>q$ under following conditions

$$
\begin{gathered}
h<\frac{\sigma^{2}}{\left|r-q-\frac{\sigma^{2}}{2}\right|}, \quad r \neq q+\frac{\sigma^{2}}{2}, \\
k<\frac{h^{2}}{\sigma^{2}+r h^{2}},
\end{gathered}
$$

Note that if $r=q+\frac{\sigma^{2}}{2}$, then under the condition 2.65, coefficients $a_{1}, a_{2}$ and $a_{3}$ are positive.

From 2.62 the numerical free boundary $S_{f}^{n}$ is increasing if $d^{n}>1$. The case $n=0$ deserves a special treatment because of the initial conditions 2.49), 2.50): $c_{j}^{0} \geq 0$ and $c_{j}^{0} \geq c_{j+1}^{0}$. For the sake of clarity in the proof that $d^{0}>1$ we distinguish two cases: $r \leq q$ and $r>q$. 
In the case $r \leq q$, the initial value of the free boundary $S_{f}(0)=1$. From $(2.63)$, (2.64) and initial conditions 2.49)

$$
d^{0}=\frac{\alpha}{\beta}=\frac{1+\frac{r h^{2}}{\sigma^{2}}}{1-h+\left(\frac{q}{\sigma^{2}}+\frac{1}{2}\right) h^{2}}>1,
$$

if

$$
\left(\frac{q-r}{\sigma^{2}}+\frac{1}{2}\right) h<1 .
$$

In the case $r>q$, from the initial conditions 2.49), 2.50) and 2.61) it follows that

$$
d^{0}=\frac{\phi(h, k)}{\psi(h, k)}
$$

where

$$
\begin{gathered}
\phi(h, k)=a_{1}\left(\frac{r}{q}-1\right)+a_{2}\left(\frac{r}{q} e^{-h}-1\right)+a_{3}\left(\frac{r}{q} e^{-2 h}-1\right)+\frac{r\left(e^{-2 h}-1\right)}{2 q h}-\alpha, \\
\psi(h, k)=\frac{r\left(e^{-2 h}-1\right)}{2 q h}-\beta \frac{r}{q}
\end{gathered}
$$

By using Taylor's expansion of $e^{-2 h}$, for $h>0$ one gets

$$
1-2 h+2 h^{2}-\frac{4}{3} h^{3}<e^{-2 h}<1-2 h+2 h^{2}-\frac{4}{3} h^{3}+\frac{2}{3} h^{4} .
$$

Note that from 2.68$)$ and 2.61$) \psi(h, k)$ satisfies

$$
\psi(h, k)<\frac{r}{q}\left(h^{2}\left(\frac{q}{\sigma^{2}}-\frac{1}{6}\right)+\frac{h^{3}}{3}\right), \quad h>0 .
$$

For the case $6 q<\sigma^{2}$, and for small enough value of $h$, satisfying

$$
h<3\left(\frac{1}{6}-\frac{q}{\sigma^{2}}\right)
$$

the right hand side of 2.69) as well as denominator $\psi(h, k)$ are negative.

For remaining case $6 q \geq \sigma^{2}$ positivity of $\psi(h, k)$ is necessary. From 2.68 and 2.49 one gets

$$
\psi(h, k)>\frac{r}{q} h^{2}\left(\frac{q}{\sigma^{2}}-\frac{1}{6}\right) \geq 0, \quad h>0 .
$$

Proving $d^{0}=\frac{\phi(h, k)}{\psi(h, k)}>1$ is equivalent to 


$$
\psi(h, k)(\phi(h, k)-\psi(h, k))>0 .
$$

Let us write the difference

$$
\begin{aligned}
\phi(h, k)-\psi(h, k) & =a_{1}\left(\frac{r}{q}-1\right)+a_{2}\left(\frac{r}{q} e^{-h}-1\right)+a_{3}\left(\frac{r}{q} e^{-2 h}-1\right)-\alpha+\beta \frac{r}{q} \\
& =\frac{r}{q}\left(a_{1}+a_{2} e^{-h}+a_{3} e^{-2 h}-1+h-\frac{h^{2}}{2}+q k\right) .
\end{aligned}
$$

Note that condition 2.72 means that the sign of both factors of the left hand side of (2.72) must have the same sign. To this purpose it is convenient to consider the following Taylor's expansion

$$
\begin{gathered}
e^{-h}=1-h+\frac{h^{2}}{2}-\frac{h^{3}}{6}+O\left(h^{4}\right), \\
e^{-2 h}=1-2 h+2 h^{2}-\frac{4}{3} h^{3}+O\left(h^{4}\right) .
\end{gathered}
$$

Then from 2.65) and (2.73)- 2.74) one gets

$$
\phi(h, k)-\psi(h, k)=\frac{r}{q}\left(q k h-\frac{h^{3}}{6}\right)+O\left(h^{4}\right) .
$$

In the case $6 q<\sigma^{2}$ the denominator $\psi(h, k)$ is negative under condition 2.70). Thus the difference 2.75 must be negative. Note that this holds true if $k<\frac{h^{2}}{6 q}$. This last condition together with (2.65) implies that

$$
k<\min \left\{\frac{h^{2}}{6 q}, \frac{h^{2}}{\sigma^{2}+r h^{2}}\right\},
$$

and as $6 q<\sigma^{2}$ condition 2.76 means

$$
k<\frac{h^{2}}{\sigma^{2}+r h^{2}} .
$$

For the case $6 q>\sigma^{2}$ the denominator $\psi(h, k)$ is positive for any $h>0$ and the difference 2.75 must be also positive or equivalently $k>\frac{h^{2}}{6 q}$. Hence, from 2.65) one concludes

$$
\frac{h^{2}}{6 q}<k<\frac{h^{2}}{\sigma^{2}+r h^{2}} .
$$


Note, that left hand side of (2.77) is smaller than right hand side if and only if

$$
h<\sqrt{\frac{6 q-\sigma^{2}}{r}} .
$$

Finally, for the case $6 q=\sigma^{2}$, from 2.71 we could not guarantee $d^{0}>1$, since there no exists step size $h$ satisfying (2.78).

Summarizing the following result has been established:

Lemma 2.2.1. Let $d^{0}$ be defined by (2.66) - 2.67) and let us assume that $6 q \neq \sigma^{2}$ and that step sizes $h, k$ are small enough and satisfy (2.64), (2.65). Then $d^{0}>1$ under the following conditions and cases:

1. if $6 q<\sigma^{2}$ and $h<3\left(\frac{1}{6}-\frac{q}{\sigma^{2}}\right)$;

2. if $6 q>\sigma^{2}$ and $\frac{h^{2}}{6 q}<k, h<\sqrt{\frac{6 q-\sigma^{2}}{r}}$.

Note that from the boundary conditions one gets

$$
S_{f}^{1}=d^{0} S_{f}^{0}>1 ; c_{0}^{1}=d^{0} S_{f}^{0}-1>0 ; c_{1}^{1}=\left\{\begin{array}{l}
0, \quad r \leq q, \\
\alpha-\beta d^{0} S_{f}^{0}, \quad r>q .
\end{array}\right.
$$

From 2.58) and 2.61) in both cases $c_{1}^{1}<c_{0}^{1}$. Furthermore, for $r \leq q$ every $c_{j}^{1}=0$ for $j=2, . . M-1$. For $r>q$, initial function $g(x)$, defined by 2.50$)$, is a convex one and then it satisfies (see [8])

$$
g\left(t x_{j-2}+(1-t) x_{j+1}\right) \leq \operatorname{tg}\left(x_{j-2}\right)+(1-t) g\left(x_{j+1}\right), \quad 0 \leq t \leq 1 .
$$

Note that $c_{j}^{0}=g\left(x_{j}\right)$, and choosing $t=\frac{2}{3}$ and $t=\frac{1}{3}$ from 2.79 one gets

$$
c_{j-1}^{0} \leq \frac{2}{3} c_{j-2}^{0}+\frac{1}{3} c_{j+1}^{0}, \quad c_{j}^{0} \leq \frac{1}{3} c_{j-2}^{0}+\frac{2}{3} c_{j+1}^{0} .
$$

Summarizing, it follows that

$$
c_{j-1}^{0}+c_{j}^{0} \leq c_{j-2}^{0}+c_{j+1}^{0},
$$

that proves

$$
\begin{aligned}
c_{j}^{1}-c_{j-1}^{1} & =a\left(c_{j-1}^{0}-c_{j-2}^{0}\right)+b\left(c_{j}^{0}-c_{j-1}^{0}\right)+f\left(c_{j+1}^{0}-c_{j}^{0}\right) \\
& -\frac{d^{0}-1}{2 h}\left(c_{j+1}^{0}-c_{j-1}^{0}-c_{j}^{0}+c_{j-2}^{0}\right) \leq 0, \quad j=2, \ldots, M-1 .
\end{aligned}
$$


Note that $c_{M}^{1}-c_{M-1}^{1} \leq 0$, since from 2.56) $c_{M-1}^{1} \geq 0$, and from the boundary conditions $c_{M}^{1}=0$. From the previous comments $\left\{c_{j}^{1}\right\}$ is a positive and nonincreasing sequence.

Following the induction arguments let us assume that for index $n-1$

$$
d^{n-1}>1, \quad S_{f}^{n}>S_{f}^{0}, \quad c_{j}^{n} \geq 0, \quad c_{j}^{n} \geq c_{j+1}^{n}, \quad j=0, . . M-1 .
$$

In order to prove that $d^{n}>1$ for $n>1$, note that the approximation of the spacial derivatives in 2.55 are $O\left(h^{2}\right)$, so one can use boundary conditions (2.58) and expression (2.60) to perform the Taylor's expansion of second order for the function $c(x, \tau)$ in the point $x_{2}$ around zero for the estimation of the value $c_{2}^{n}$ as

$$
c_{2}^{n}=-1-\frac{4 r h^{2}}{\sigma^{2}}+\left(1-2 h+\frac{4 q h^{2}}{\sigma^{2}}+2 h^{2}\right) S_{f}^{n} .
$$

In Example 2.2.2 we will show numerical justification of this assumption.

Denoting denominator of $d^{n}$ in 2.63) as $C_{1}(n)$ and using (2.81) one gets

$$
C_{1}(n)=\frac{2 h}{\sigma^{2}}\left(q S_{f}^{n}-r\right)+\frac{h^{2}}{2 \sigma^{2}}\left(\sigma^{2}+2 q\right) S_{f}^{n},
$$

that is positive since $S_{f}^{n}>\frac{r}{q}$ (for $r>q$ ) or $S_{f}^{n}>1$ (for $r \leq q$ ).

Since $C_{1}(n)>0$ and from 2.63, 2.57) and 2.82)

$$
d^{n}-1=\frac{k h C_{2}(n)-k h^{2}\left(\sigma^{2} r S_{f}^{n}+2 r\left(q S_{f}^{n}-r\right)\right)}{2 \sigma^{2} C_{1}(n)},
$$

where

$$
C_{2}(n)=\left(\sigma^{4}+4 q^{2}-4 q r+4 q \sigma^{2}\right) S_{f}^{n}+4 r\left(r-q-\frac{\sigma^{2}}{2}\right)=\lambda_{1} S_{f}^{n}+\lambda_{2} .
$$

It can be shown, that 2.84 is positive for $r<q+\sigma^{2}+\frac{\sigma^{4}}{4 q}$, i.e. $\lambda_{1}>0$, since $S_{f}^{n}>1$ in that case and $\lambda_{1}>\left|\lambda_{2}\right|$. If $\lambda_{1}=0$, then $\lambda_{2}>0$. For the case $r-\left(q+\sigma^{2}+\frac{\sigma^{4}}{4 q}\right)=\delta>0$ one gets

$$
-\delta S_{f}^{n}+\lambda_{2}>0, \quad \text { if } \quad S_{f}^{n}<\frac{4 r}{\delta}\left(r-q-\frac{\sigma^{2}}{2}\right),
$$

that is fulfilled since $\delta$ is small. It is not difficult to show that the restriction on $S_{f}^{n}$ is weaker than the Samuelson's estimation (see [72]) It means that $C_{2}(n)>0$. Consequently, from 2.83 $d^{n}>1$ for small enough values of $h$ and $k$. 
Let us denote

$$
\begin{aligned}
y(z) & =1+\frac{k h\left(\left(\sigma^{4}+4 q^{2}-4 q r+4 q \sigma^{2}\right) z+4 r\left(r-q-\frac{\sigma^{2}}{2}\right)\right)}{2 \sigma^{2} \frac{2 h}{\sigma^{2}}(q z-r)+\frac{h^{2}}{2 \sigma^{2}}\left(\sigma^{2}+2 q\right) z} \\
& -\frac{k h^{2}\left(\sigma^{2} r z+2 r(q z-r)\right)}{2 \sigma^{2} \frac{2 h}{\sigma^{2}}(q z-r)+\frac{h^{2}}{2 \sigma^{2}}\left(\sigma^{2}+2 q\right) z},
\end{aligned}
$$

with negative derivative

$$
\frac{d y}{d z}=-\frac{2 r k\left(\left(\sigma^{2}+2 q\right)\left(2 \sigma^{2}-h\left(\sigma^{2}+2 q\right)\right)+h\left(\sigma^{2} r h+4 q r+2 q r h\right)\right)}{\left(\left(\sigma^{2} h+4 q+2 q h\right) z-4 r\right)^{2}}
$$

for small enough values of $h$. Since $y\left(S_{f}^{n}\right)=d^{n}$, and $S_{f}^{n}>S_{f}^{n-1}$, then $d^{n}$ is a decreasing discrete function of $n$. That means that $\left\{S_{f}^{n}\right\}$ has a concave behaviour.

In order to show the positivity of the numerical solution $\left\{c_{j}^{n}\right\}$ we prove that coefficients $a_{1}^{n}$ and $a_{2}^{n}$ in (2.56) are positive. Firstly, since $d^{n} \geq 1$,

$$
a_{1}^{n}=a+\frac{d^{n}-1}{2 h}>0 .
$$

From 2.82 and 2.83) the difference $d^{n}-1=O(k)$ and because $2 h f=O\left(\frac{k}{h}\right)$ from (2.57), then $2 h f>d^{n}-1$ for small enough values of $h$, that means $a_{2}^{n}$ is positive.

Under assumption (2.80) together with positivity of coefficients $a_{1}^{n}$ and $a_{2}^{n}$ and under conditions 2.64, 2.65 the positivity of $\left\{c_{j}^{n+1}\right\}$ is proved. Moreover, $\left\{c_{j}^{n+1}\right\}$ is a non-increasing sequence with respect to index $j$ from 2.56, since

$$
\begin{aligned}
c_{j+1}^{n+1}-c_{j}^{n+1} & =a_{1}^{n} c_{j}^{n}+b c_{j+1}^{n}+a_{2}^{n} c_{j+2}^{n}-a_{1}^{n} c_{j-1}^{n}-b c_{j}^{n}-a_{2}^{n} c_{j+1}^{n}= \\
& =a_{1}^{n}\left(c_{j}^{n}-c_{j-1}^{n}\right)+b\left(c_{j+1}^{n}-c_{j}^{n}\right)+a_{2}^{n}\left(c_{j+2}^{n}-c_{j+1}^{n}\right) \leq 0 .
\end{aligned}
$$

Now the following result can be established:

Theorem 2.2.1. Let $\left\{c_{j}^{n}, S_{f}^{n}\right\}$ be the numerical solution of scheme (2.56) for the transformed American call option problem (2.48) and let $d^{n}$ be defined by (2.63). Then under conditions (2.64), (2.65) and Lemma 2.2.1 the numerical scheme (2.56) guarantees the following properties of the numerical solution:

1. Increasing monotonicity and positivity of values $S_{f}^{n}, \quad n=0, \ldots, N$ with concave behaviour; 
2. Non-negativity of the vectors $\left\{c_{j}^{n}\right\}, \quad n=0, \ldots, N$;

3. Non-increasing monotonicity of the vectors $\left\{c_{j}^{n}\right\}$ with respect to spacial index for each fixed $n=0, \ldots, N$.

Further the study of the stability and the consistency of the scheme will be studied. Let us denote the numerical solution vector $c^{n}=\left[\begin{array}{llll}c_{0}^{n} & c_{1}^{n} & \ldots & c_{M}^{n}\end{array}\right] \in \mathbb{R}^{M+1}$.

Theorem 2.2.2. Under assumptions of Theorem 2.2.1 the numerical scheme (2.56) for solving transformed problem (2.48)-(2.53) is stable.

Proof. Since $c_{j}^{n}$ is non-decreasing vectors for each fixed $n$ and from the boundary conditions 2.58)

$$
\left\|c^{n}\right\|_{\infty}=c_{0}^{n}=S_{f}^{n}-1,
$$

where $S_{f}^{n}$ is positive non-decreasing vector. Therefore, from $(2.62)$, it is clear that

$$
\max _{n} S_{f}^{n}=S_{f}^{N}=\prod_{n=0}^{N-1} d^{n} S_{f}^{0}
$$

It was shown in Theorem 2.2.1 that $d^{n}>1$ and it is decreasing in time, moreover, $d^{n}=1+O(k)$ for any $n \geq 1$. Then there exists $\eta>0$ such that $d^{n}<1+\eta k$, and one gets

$$
\prod_{n=1}^{N} d^{n}<\left(d^{1}\right)^{N-1}<\left(d^{1}\right)^{N}=\left(1+\frac{T \eta}{N}\right)^{N}<e^{T \eta}
$$

Thus, from 2.85) - 2.87) it follows that

$$
\left\|c^{n}\right\|_{\infty}=\max _{n} S_{f}^{n}-1<e^{T \eta} d^{0} S_{f}^{0}-1
$$

and the scheme is $\|\cdot\|_{\infty}$-stable by the definition.

Now let us study consistency of the numerical scheme. Assume that $c(x, \tau)$ is continuously differentiable four times with respect to $x$ and two times with respect to $\tau$, and there exists the second derivative of $S_{f}(\tau)$. Using Taylor's expansion 


\section{LINEAR BLACK-SCHOLES MODEL FOR AMERICAN OPTIONS}

about $\left(x_{j}, \tau^{n}\right)$ and following an analogous procedure of the previous chapter, one gets that the local truncation error

$$
T_{j}^{n}\left(\tilde{c}, \tilde{S}_{f}\right)=O\left(h^{2}\right)+O(k)
$$

For study of consistency of the numerical solution of the free boundary let us rewrite boundary conditions in the following form

$$
\begin{gathered}
L_{1}\left(c, S_{f}\right)=c(0, \tau)+1-S_{f}(\tau)=0, \quad L_{2}\left(c, S_{f}\right)=\frac{\partial x}{\partial x}(0, \tau)-S_{f}(\tau)=0, \\
L_{3}\left(c, S_{f}\right)=\frac{\sigma^{2}}{2} \frac{\partial^{2} c}{\partial x^{2}}(0, \tau)-\left(q+\frac{\sigma^{2}}{2}\right) S_{f}(\tau)+r=0 .
\end{gathered}
$$

In accordance with expressions 2.58 and 2.59 finite difference approximations for the boundary conditions are

$$
\begin{gathered}
F_{1}\left(c^{n}, S_{f}^{n}\right)=c_{0}^{n}+1-S_{f}^{n}=0, \quad F_{2}\left(c^{n}, S_{f}^{n}\right)=\frac{c_{1}^{n}-c_{-1}^{n}}{2 h}-S_{f}^{n}=0, \\
F_{3}\left(c^{n}, S_{f}^{n}\right)=\frac{\sigma^{2}}{2} \frac{c_{-1}^{n}-2 c_{0}^{n}+c_{1}^{n}}{h^{2}}-\left(q+\frac{\sigma^{2}}{2}\right) S_{f}^{n}+r=0 .
\end{gathered}
$$

Note that $F_{1}\left(\tilde{c}^{n}, \tilde{S}_{f}^{n}\right)=L_{1}\left(\tilde{c}^{n}, \tilde{S}_{f}^{n}\right)$, and truncation error satisfies

$$
F_{2}\left(\tilde{c}, \tilde{S}_{f}^{n}\right)-L_{2}\left(\tilde{c}, \tilde{S}_{f}^{n}\right)=O\left(h^{2}\right), \quad F_{3}\left(\tilde{c}, \tilde{S}_{f}^{n}\right)-L_{3}\left(\tilde{c}, \tilde{S}_{f}^{n}\right)=O\left(h^{2}\right)
$$

The truncation error of the approximation of the boundary condition behaves as $h^{2}$. Thus, the following theorem is established.

Theorem 2.2.3. Assuming that the solution of the PDE problem (2.48)-(2.53) admits two times continuous partial derivative with respect to time and up to order four with respect to space, the numerical solution computed by the scheme is consistent with the equation (2.48) and boundary conditions of order two in space and order one in time.

\subsubsection{Numerical examples}

In this section numerical value of the free boundary obtained by the proposed method is compared with other techniques. 
2.2 Front-fixing method for American call option

\begin{tabular}{|c|c|c|c|c|c|c|}
\hline Asset Price & True Value & GL & LUBA & HW & OS & FF \\
\hline 80 & 0.2194 & 0.2185 & 0.2195 & 0.2193 & 0.2193 & 0.2196 \\
90 & 1.3864 & 1.3851 & 1.3862 & 1.3858 & 1.3858 & 1.3868 \\
100 & 4.7825 & 4.7835 & 4.7821 & 4.7816 & 4.7817 & 4.7827 \\
110 & 11.0978 & 11.1120 & 11.0976 & 11.0969 & 11.0971 & 11.0981 \\
120 & 20.0004 & 20.0000 & 20.0000 & 20.0005 & 20.0000 & 20.0006 \\
\hline \multicolumn{2}{|c|}{ RMSE } & $6.4078-3$ & $2.8636-4$ & $6.3246-4$ & $5.7619-4$ & $2.5391-4$ \\
\hline
\end{tabular}

Table 2.4: American call option values calculated by the proposed front-fixing method (FF) and other methods.

Example 2.2.1. We consider the American call option pricing problem (2.5)-(2.7) with the parameters

$$
r=0.03, \quad q=0.07, \quad \sigma=0.2, \quad T=0.5, \quad E=100 .
$$

The proposed method (FF) with step sizes $h=10^{-3}, k=2.5 \cdot 10^{-5}$ is compared with other approaches presented in [96]: Gauss-Laguerre (GL) method of Frontczak and Schobel [49]; the lower and upper bound approximation (LUBA) of Broadie and Detemple [17]; the Han-Wu (HW) method [54]; operator splitting (OS) of Ikonen and Toiwanen [60]. The root-mean-square error (RMSE) is used to measure the accuracy of the method. The comparison presented in Table 2.4 shows that proposed scheme produces similar results with the advantages of the explicitness, positivity and monotonicity.

Free boundary obtained in the example below is compared with analytical approximation closed to maturity presented in [46].

Example 2.2.2. Let us consider the problem (2.5)-(2.7) with the following parameters

$$
r=0.1, \quad q=0.05, \quad \sigma=0.2, \quad T=1, \quad E=10 .
$$

The position of the free boundary at $\tau=T$ is $B(T)=22.3754$ (in [109]) and it was computed by the proposed method as $S_{f}(T)=2.2375$, since the transformed problem is dimensionless, then $B(T)=22.375$. The free boundary motion for the problem is compared with analytical approximation [46]. Results are presented on Figure 2.2. One can see that the qualitative properties of the exact solution are preserved with the proposed scheme: the free boundary value is positive, increasing in time with concave behaviour. In Table 2.5 a comparison of option values 


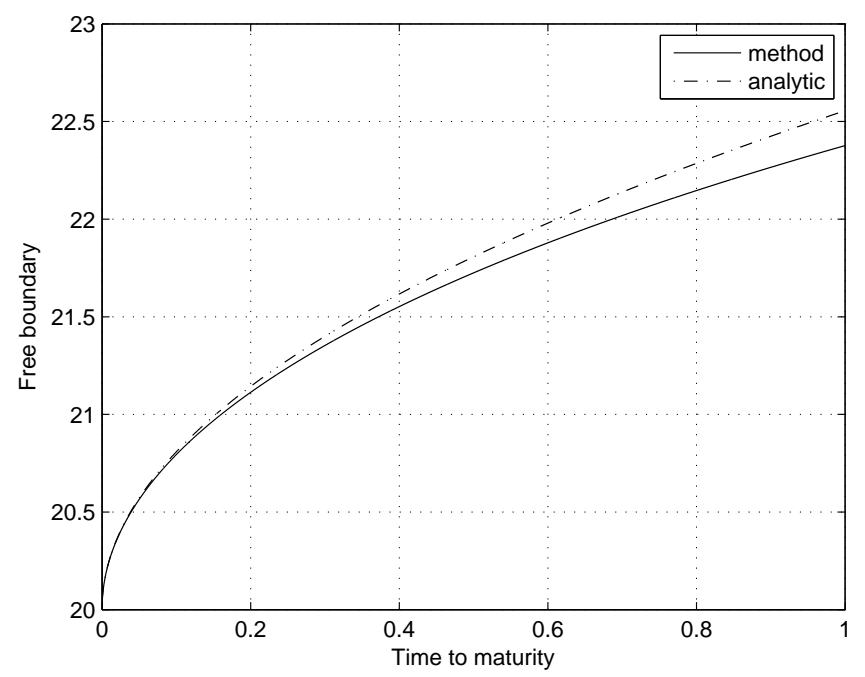

Figure 2.2: Analytical ("analytic") and computed by the proposed method ("method") values of optimal stopping boundaries in Example 2.2.2.

obtained by the proposed front-fixing method and by other methods such as semiexplicit formula, presented in [109], trinomial tree, finite difference approximation and analytical approximation of Barone-Adesi and Whaley [7] is presented.

For the theoretical study the Taylor's expansion is used for estimating $c_{2}^{n}$. The difference between computed value and estimation (2.81) is shown in Figure 2.3 . justifying reliability of this assumption.

Example 2.2.3. In order to compare computational efficiency, we consider the

\begin{tabular}{|c|c|c|c|c|c|}
\hline Method / The asset value S & 15 & 18 & 20 & 21 & 22.375 \\
\hline Ševčovič's method & 5.15 & 8.09 & 10.03 & 11.01 & 12.37 \\
Trinomial tree & 5.15 & 8.09 & 10.03 & 11.01 & 12.37 \\
Finite differences & 5.49 & 8.48 & 10.48 & 11.48 & 12.48 \\
Analytical approximation & 5.23 & 8.10 & 10.04 & 11.02 & 12.38 \\
Proposed method & 5.21 & 8.09 & 10.03 & 11.01 & 12.37 \\
\hline
\end{tabular}

Table 2.5: American call option values with parameters 2.90 calculated by various methods. 


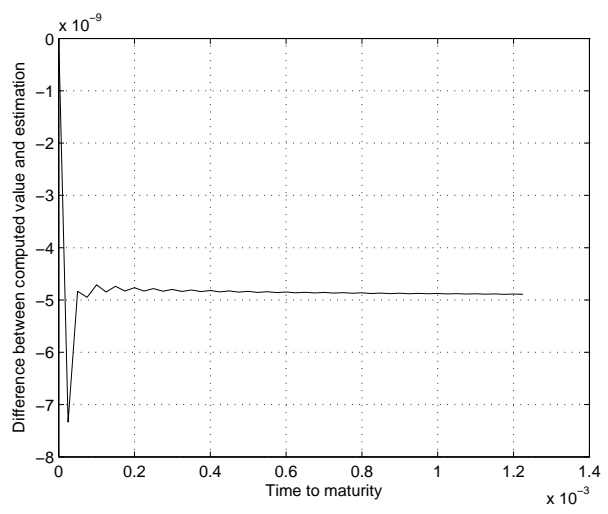

Figure 2.3: The difference between computed value and estimation of $c_{2}^{n}$ for the problem with the parameters 2.90 and step sizes $h=10^{-3}$ and $k=6.25 \cdot 10^{-6}$.

problem with the parameters [54]:

$$
r=0.03, \quad q=0.03, \quad \sigma=0.4, \quad T=0.5, \quad E=100 .
$$

Table 2.6 shows the comparison of the proposed method with other methods in [54]. Since exact values are not known, the results of the binomial method with large steps (15000) are used for "True Value". FDP stands for the CrankNicolson finite-difference method with projected SOR iteration to impose the free boundary condition. FDE stands for the Crank-Nicolson finite difference method with elimination-back substitution. HW stands for the Han and Wu method (see [54]). FF stands for the proposed explicit finite difference method combined with the front-fixing transformation with stepsizes $h=10^{-3}$ and $k=6.25 \cdot 10^{-6}$.

From the results presented in Table 2.6 we can conclude that the proposed method is competitive and effective, since it produces the similar error with smaller computational time.

The results of this section have been presented in ECMI conference 2014.

Since stability of the method based on the front-fixing transformation 2.47) depends on parameters and require strict conditions on step sizes, an alternative transformation will be proposed in the following section. 


\begin{tabular}{|c|c|c|c|c|c|}
\hline Asset Price & True Value & FDP & FDE & HW & FF \\
\hline 40 & 0.002792 & 0.0025 & 0.0025 & 0.0028 & 0.0028 \\
\hline 50 & 0.045594 & 0.0457 & 0.0457 & 0.0457 & 0.0455 \\
\hline 60 & 0.301387 & 0.3014 & 0.3015 & 0.3015 & 0.3012 \\
\hline 70 & 1.145799 & 1.1459 & 1.1461 & 1.1461 & 1.1456 \\
\hline 80 & 3.041536 & 3.0414 & 3.0415 & 3.0415 & 3.0414 \\
\hline 90 & 6.328677 & 6.3285 & 6.3287 & 6.3287 & 6.3283 \\
\hline 100 & 11.108407 & 11.1066 & 11.1068 & 11.1068 & 11.1078 \\
\hline 110 & 17.266726 & 17.2664 & 17.2665 & 17.2665 & 17.2663 \\
\hline 120 & 24.564972 & 24.5654 & 24.5655 & 24.5655 & 24.5644 \\
\hline \multicolumn{2}{|c|}{ RMSE } & $6.4217-4$ & $5.8822-4$ & $5.8012-4$ & $3.5593-4$ \\
\hline \multicolumn{2}{|c|}{ CPU-time, sec } & 37.130 & 15.760 & 11.500 & 6.813 \\
\hline
\end{tabular}

Table 2.6: Computational efficiency of various methods in Example 2.2.3. 


\subsection{New efficient front-fixing method for American op- tion pricing}

In this section a new front-fixing transformation for American call option on dividendpaying assets is proposed. Under this transformation a nonlinear PDE with homogeneous boundary conditions independent of the free boundary is obtained. This fact simplifies the numerical analysis of the finite difference scheme.

Let us consider the dimensionless transformation with two goal: to fix the computational domain as in [112] and to simplify the boundary conditions like [111], p. 122 ,

$$
x=\ln \frac{B(\tau)}{S}, \quad c(x, \tau)=\frac{C(S, \tau)-S+E}{E}, \quad S_{f}(\tau)=\frac{B(\tau)}{E} .
$$

Under transformation (2.92) the problem (2.5) - 2.7) can be rewritten in normalized form

$$
\frac{\partial c}{\partial \tau}=\frac{\sigma^{2}}{2} \frac{\partial^{2} c}{\partial x^{2}}-\left(r-q-\frac{\sigma^{2}}{2}+\frac{S_{f}^{\prime}}{S_{f}}\right) \frac{\partial c}{\partial x}-r c-q S_{f} e^{-x}+r, \quad x>0,0<\tau \leq T,
$$

with new initial conditions

$$
\begin{gathered}
S_{f}(0)=\max \left(\frac{r}{q}, 1\right), \quad c(x, 0)=\left\{\begin{array}{ll}
1-e^{-x}, & r \leq q, \\
g(x), & r>q,
\end{array} \quad x \geq 0,\right. \\
g(x)=\max \left(1-\frac{r}{q} e^{-x}, 0\right) .
\end{gathered}
$$

Boundary conditions are transformed into the following

$$
\begin{aligned}
\frac{\partial c}{\partial x}(0, \tau) & =0, \\
c(0, \tau) & =0, \\
\lim _{x \rightarrow \infty} c(x, \tau) & =1,
\end{aligned}
$$

As in previous section, we assume that (2.93) holds true at $x=0$, such that

$$
\frac{\sigma^{2}}{2} \frac{\partial^{2} c}{\partial x^{2}}\left(0^{+}, \tau\right)-q S_{f}(\tau)+r=0
$$




\section{LINEAR BLACK-SCHOLES MODEL FOR AMERICAN OPTIONS}

The problem (2.93)-2.97) can be numerically studied on the fixed domain $\left[0, x_{\max }\right] \times[0, \tau]$. The value $x_{\max }$ is chosen big enough to guarantee the boundary condition. The computational grid of $M+1$ spatial points and $N+1$ time levels is chosen as in the previous cases (2.13)-(2.14).

The approximate value of option price at the point $x_{j}$ and time $\tau^{n}$ is denoted by $c_{j}^{n} \approx c\left(x_{j}, \tau^{n}\right)$ and the approximate value of the free boundary is denoted by $S_{f}^{n} \approx S_{f}\left(\tau^{n}\right)$. Then a forward two-time level and centred in a space explicit scheme is constructed for internal spatial nodes as follows

$$
c_{j}^{n+1}=a_{1}^{n} c_{j-1}^{n}+b c_{j}^{n}+a_{2}^{n} c_{j+1}^{n}+k\left(r-q S_{f}^{n} e^{-x_{j}}\right), \quad 1 \leq j \leq M-1,
$$

where

$$
\begin{gathered}
a_{1}^{n}=\frac{k}{2 h^{2}}\left(\sigma^{2}+\left(r-q-\frac{\sigma^{2}}{2}\right) h\right)+\frac{S_{f}^{n+1}-S_{f}^{n}}{2 h S_{f}^{n}}=a_{1}+\frac{S_{f}^{n+1}-S_{f}^{n}}{2 h S_{f}^{n}}, \\
b=1-\sigma^{2} \frac{k}{h^{2}}-r k, \\
a_{2}^{n}=\frac{k}{2 h^{2}}\left(\sigma^{2}-\left(r-q-\frac{\sigma^{2}}{2}\right) h\right)-\frac{S_{f}^{n+1}-S_{f}^{n}}{2 h S_{f}^{n}}=a_{2}-\frac{S_{f}^{n+1}-S_{f}^{n}}{2 h S_{f}^{n}} .
\end{gathered}
$$

From 2.96 and using the second order centred approximation of the boundary conditions (2.95) and (2.98) one gets

$$
\begin{gathered}
c_{0}^{n}=0, \quad \frac{c_{1}^{n}-c_{-1}^{n}}{2 h}=0, \\
\frac{\sigma^{2}}{2} \frac{c_{-1}^{n}-2 c_{0}^{n}+c_{1}^{n}}{h^{2}}-q S_{f}^{n}+r=0,
\end{gathered}
$$

where $c_{-1}^{n}$ means the value of the solution at the fictitious point $x=-h$, that should be eliminated later. From (2.101) and (2.102) the connection between the free boundary $S_{f}^{n}$ and option value $c_{1}^{n}$ on the same time level $n$ is presented as

$$
c_{1}^{n}=\frac{h^{2}}{\sigma^{2}}\left(q S_{f}^{n}-r\right), \quad n \geq 1 .
$$

Let us consider the right side approximation of the second order (see Table 1.1) of the spatial derivative in (2.95)

$$
\frac{\partial c}{\partial x}\left(0, \tau^{n}\right)=\frac{4 c_{1}^{n}-c_{2}^{n}-3 c_{0}^{n}}{2 h}=0, \quad n \geq 1 .
$$




\subsection{New efficient front-fixing method for American option pricing}

From (2.101) and (2.104) one gets relation between $c_{1}^{n}$ and $c_{2}^{n}$ for any $n \geq 1$

$$
c_{2}^{n}=4 c_{1}^{n} .
$$

Thus, computation of the numerical solution $c_{2}^{n}$ at point $\left(x_{2}, \tau^{n}\right)$ does not need the scheme (2.99). It means that the scheme (2.99) is not used for $j=2$. For the right boundary $\left(x=x_{\max }\right)$ from $(2.97)$ the Dirichlet's boundary condition is $c_{M}^{n}=1$ for any $n \geq 0$.

We use together (2.99) for $j=1$ and (2.103) at the time level $n+1$ to obtain the nonlinear law of the free boundary motion

$$
\begin{gathered}
S_{f}^{n+1}=d^{n} S_{f}^{n} \\
d^{n}=\frac{b c_{1}^{n}+f c_{2}^{n}+\frac{c_{2}^{n}}{2 h}+\frac{r h^{2}}{\sigma^{2}}+k\left(r-q S_{f}^{n} e^{-h}\right)}{\frac{c_{2}^{n}}{2 h}+\frac{q h^{2}}{\sigma^{2}} S_{f}^{n}} .
\end{gathered}
$$

Fully implicit finite difference scheme is also employed to solve the problem (2.93) - 2.97). In that case the computational scheme takes the following form

$$
\begin{aligned}
\frac{c_{j}^{n+1}-c_{j}^{n}}{k} & =\frac{1}{2} \sigma^{2} \frac{c_{j-1}^{n+1}-2 c_{j}^{n+1}+c_{j+1}^{n+1}}{h^{2}} \\
& -\left(r-q-\frac{\sigma^{2}}{2}+\frac{S_{f}^{n+1}-S_{f}^{n}}{k S_{f}^{n+1}}\right) \frac{c_{j+1}^{n+1}-c_{j-1}^{n+1}}{2 h} \\
& -r c_{j}^{n+1}+r-q S_{f}^{n+1} e^{-x_{j}}, \quad j=1, \ldots M-1, \\
c_{0}^{n+1}=0, \quad c_{M}^{n+1}=1, & \\
\frac{\sigma^{2}}{h^{2}} c_{1}^{n+1}-q S_{f}^{n+1}+r & =0 .
\end{aligned}
$$

Writing the finite-difference equations (2.108) and introducing the boundary conditions (2.109) and the discretization of the free boundary (2.110), a nonlinear system of equation is obtained. We denote by $Y^{l}=\left[\begin{array}{llll}c_{1}^{n+1} & \ldots & c_{M-1}^{n+1} & S_{f}^{n+1}\end{array}\right]^{T}$ vector of $M$ unknowns on the $l$-th iteration. This nonlinear system is solved by widely used Newton method and extensions [69], [78].

$$
Y^{l+1}=Y^{l}-J^{-1} F^{l},
$$

where $F^{l}$ is matrix, obtained from 2.108 and 2.110 by substituting $Y^{l} . J$ is Jacobian of the system. The iteration process is done until $\left\|Y^{l+1}-Y^{l}\right\|<\varepsilon$ for a given error tolerance $\varepsilon$. 


\subsubsection{Qualitative properties of the scheme}

In this section we will show qualitative scheme properties such as the free boundary non-decreasing monotonicity as well as the positivity and non-decreasing spacial monotonicity of the numerical option price under transformation by using the induction principle. The positivity of the coefficients $a_{1}, b$ and $a_{2}$ appearing in 2.100) will play an important role for obtaining this purpose. Note, that using expressions 2.100 it is easy to obtain that the constants of the scheme $a_{1}, b$ and $a_{2}$ are positive for both cases: $r \leq q$ and $r>q$ under following conditions

$$
\begin{gathered}
h<\frac{\sigma^{2}}{\left|r-q-\frac{\sigma^{2}}{2}\right|}, \quad r \neq q+\frac{\sigma^{2}}{2}, \\
k<\frac{h^{2}}{\sigma^{2}+r h^{2}},
\end{gathered}
$$

If $r=q+\frac{\sigma^{2}}{2}$, then under the condition 2.113 , coefficients $a_{1}, b$ and $a_{2}$ are positive.

In order to show that the numerical free boundary $S_{f}^{n}$ is increasing, from 2.106 we need to prove that $d^{n}>1$. The case $n=0$ deserves a special treatment because of the initial conditions 2.94. We have $c_{j}^{0} \geq 0$ and $c_{j}^{0} \leq c_{j+1}^{0}$.

In order to provide numerical analysis of the scheme we have to estimate value $c_{2}^{n}$ using the values on $n$-th time level. Suppose, that the solution $c(x, \tau)$ is continuously differentiable up to fourth order. Then the Taylor's expansion in the node $x_{2}$ has the following form

$$
c\left(x_{2}, \tau^{n}\right)=c\left(0, \tau^{n}\right)+2 h \frac{\partial c}{\partial x}\left(0, \tau^{n}\right)+2 h^{2} \frac{\partial^{2} c}{\partial x^{2}}\left(0, \tau^{n}\right)+\frac{4 h^{3}}{3} \frac{\partial^{3} c}{\partial x^{3}}\left(0, \tau^{n}\right)+O\left(h^{4}\right) .
$$

From boundary conditions 2.96, (2.95) approximations 2.101) and (2.102), one gets

$$
c_{2}^{n}=4 c_{1}^{n}+O\left(h^{4}\right) .
$$

For the sake of clarity in order to prove that $d^{0}>1$ and $\left\{c_{j}^{1}\right\}$ is an increasing sequence we distinguish two cases: $r>q$ and $r \leq q$.

Case $r>q$.

From the initial conditions 2.94) it follows that

$$
d^{0}=1+\frac{\sigma^{2} k}{h^{2}}\left(1-e^{-h}\right)>1 .
$$




\subsection{New efficient front-fixing method for American option pricing}

Note that from the boundary conditions 2.96 and expressions 2.103, , 2.105) one gets

$$
c_{1}^{1}=\frac{r h^{2}}{\sigma^{2}}\left(\frac{q}{r} S_{f}^{1}-1\right)>c_{0}^{1}=0, \quad c_{2}^{1}=4 c_{1}^{1}+O\left(h^{4}\right)>c_{1}^{1} .
$$

From initial conditions (2.94), the values of the solution at interior mesh points are

$$
\begin{gathered}
c_{j}^{1}-c_{j-1}^{1}=a\left(c_{j-1}^{0}-c_{j-2}^{0}\right)+b\left(c_{j}^{0}-c_{j-1}^{0}\right)+f\left(c_{j+1}^{0}-c_{j}^{0}\right) \\
-\frac{d^{0}-1}{2 h}\left(c_{j+1}^{0}-c_{j-1}^{0}-c_{j}^{0}+c_{j-2}^{0}\right)+r k e^{-j h}\left(e^{h}-1\right), \quad j=3, \ldots, M-1 .
\end{gathered}
$$

Note that $c(x, 0)$, defined by $(2.94)$, is a concave function for $x_{j-1} \geq \ln \frac{r}{q}$ and consequently verifies ( see [8])

$$
g\left(t x_{j-2}+(1-t) x_{j+1}\right) \geq \operatorname{tg}\left(x_{j-2}\right)+(1-t) g\left(x_{j+1}\right) .
$$

Since $c_{j}^{0}=g\left(x_{j}\right)$ by choosing $t=\frac{2}{3}$ and $t=\frac{1}{3}$ for the condition 2.116 one gets

$$
c_{j-1}^{0} \geq \frac{2}{3} c_{j-2}^{0}+\frac{1}{3} c_{j+1}^{0}, \quad c_{j}^{0} \geq \frac{1}{3} c_{j-2}^{0}+\frac{2}{3} c_{j+1}^{0} .
$$

If $x_{j+1} \leq \ln \frac{r}{q}$ function $c(x, 0)$ is a constant. If $x_{j} \leq \ln \frac{r}{q}<x_{j+1}$ or $x_{j-1} \leq$ $\ln \frac{r}{q}<x_{j}$, then

$$
c_{j-1}^{0}+c_{j}^{0} \geq c_{j-2}^{0}+c_{j+1}^{0} .
$$

Summarizing all possible cases, (2.117) holds true and from 2.115 it follows that $c_{j}^{1}-c_{j-1}^{1} \geq 0, j=3, \ldots, M-1$.

From the scheme 2.99 for $j=M-1$, since $\left\{c_{j}^{0}\right\}$ is increasing, one gets

$$
c_{M-1}^{1} \leq(1-r k) c_{M}^{0}+k\left(r-q S_{f}^{0} e^{-x_{M-1}}\right)=1-k q S_{f}^{0} e^{-x_{M-1}} \leq c_{M}^{1}=1 .
$$

Case $r \leq q$

In that case initial conditions (2.94) are different, then $d^{0}$ has the form

$$
\begin{aligned}
d^{0}-1 & =\frac{b\left(1-e^{-h}\right)+f\left(1-e^{-2 h}\right)+k\left(r-q e^{-h}\right)+\frac{(r-q) h^{2}}{\sigma^{2}}}{\frac{1-e^{-2 h}}{2 h}+\frac{q h^{2}}{\sigma^{2}}} \\
& \geq \frac{h(1+(q-r) k)+k \frac{\sigma^{2}}{2}}{\frac{1-e^{-2 h}}{2 h}+\frac{q h^{2}}{\sigma^{2}}}=O(h),
\end{aligned}
$$


since

$$
e^{-j h}<1-j h, \quad \frac{1-e^{-2 h}}{2 h}>1 .
$$

It means that $S_{f}^{1}>S_{f}^{0}=1$, then from boundary conditions 2.103, 2.105, $c_{2}^{1}=4 c_{1}^{1}>c_{1}^{1}>c_{0}^{1}=0$. For $j>2$ one gets $c_{j+1}^{1} \geq c_{j}^{1}$ since initial function is concave for any $x \in\left[0 ; x_{\max }\right]$.

Assume, that for any $n>1$

$$
d^{n-1}>1 ; \quad c_{j}^{n} \geq 0, j=0, . . M ; \quad c_{j}^{n} \leq c_{j+1}^{n}, j=0, . . M-1 .
$$

Let us prove, that $d^{n}>1$.

$$
d^{n}=\frac{b c_{1}^{n}+f c_{2}^{n}+\frac{c_{2}^{n}}{2 h}+\frac{r h^{2}}{\sigma^{2}}+k\left(r-q S_{f}^{n} e^{-h}\right)}{\frac{c_{2}^{n}}{2 h}+\frac{q h^{2}}{\sigma^{2}} S_{f}^{n}}>1 .
$$

From (2.107), denominator of $d^{n}$ is positive. To guarantee $d^{n}>1$, it is necessary that

$$
(b-1) c_{1}^{n}+f c_{2}^{n}+k\left(r-q S_{f}^{n} e^{-h}\right)>0 .
$$

Using 2.103), 2.105) and Taylor's expansion for exponent function, the lefthand side of 2.121 can be presented for small enough $k$ and $h$ in form

$$
\begin{aligned}
(b-1) c_{1}^{n} & +a_{2} c_{2}^{n}+k\left(r-q S_{f}^{n} e^{-h}\right) \\
& \geq\left(\left(b-1+4 a_{2}\right) \frac{h^{2}}{\sigma^{2}}-k\right)\left(q S_{f}^{n}-r\right)+k h q S_{f}^{n}+O\left(k h^{2}\right) \\
& \geq k h q S_{f}^{n}\left(1-\frac{2\left(r-q-\frac{\sigma^{2}}{2}\right)}{\sigma^{2}}\right)+r k h \frac{2\left(r-q-\frac{\sigma^{2}}{2}\right)}{\sigma^{2}}+O\left(k h^{2}\right) .
\end{aligned}
$$

If $r-q-\frac{\sigma^{2}}{2} \leq 0,2.122$ is positive. If $r-q-\frac{\sigma^{2}}{2}>0$, then by dividing last expression in 2.122 by $\frac{2 k h\left(r-q-\frac{\sigma^{2}}{2}\right)}{\sigma^{2}}>0$, one has to prove that

$$
q S_{f}^{n} \frac{\sigma^{2}-r+q}{r-q-\frac{\sigma^{2}}{2}}+r>0 .
$$

If $r-q-\sigma^{2} \leq 0$ is fulfilled. Otherwise it holds true if

$$
S_{f}^{n}<\frac{r\left(r-q-\frac{\sigma^{2}}{2}\right)}{q\left(r-q-\sigma^{2}\right)}=\frac{r}{q}\left(1+\frac{\sigma^{2}}{2\left(r-q-\sigma^{2}\right)}\right) .
$$




\subsection{New efficient front-fixing method for American option pricing}

Let us denote the critical asset price for perpetual American calls and puts respectively by $S_{f}^{+}(\infty)$ and $S_{f}^{-}(\infty)$, see [72],

$$
S_{f}^{+}(\infty)=\frac{\alpha_{+}}{\alpha_{+}-1}, \quad S_{f}^{-}(\infty)=\frac{\alpha_{-}}{\alpha_{-}-1},
$$

where

$$
\alpha_{ \pm}=\frac{1}{2 \sigma^{2}}\left(\sigma^{2}-2(r-q) \pm \sqrt{4(r-q)^{2}+4(r+q)^{2} \sigma^{2}+\sigma^{4}}\right) .
$$

If we consider polynomial $F(x)=\left(x-\alpha_{-}\right)\left(x-\alpha_{+}\right)$and value

$$
\alpha_{*}=\frac{S_{f}^{*}}{S_{f}^{*}-1},
$$

where $S_{f}^{*}$ is equal to right-hand side of inequality (2.123), then

$$
F\left(\alpha_{*}\right)=-\frac{2 \sigma^{2} q r\left(2 r-q-\sigma^{2}\right)}{\left(2(r-q)^{2}+\sigma^{2}(2 q-r)^{2}\right)^{2}}<0 .
$$

Since $\alpha_{-}<\alpha_{+}$and both are roots of convex polynomial $F(x)$, then from (2.126), it is clear that $\alpha_{*}<\alpha_{+}$. Using definitions 2.124) and 2.125, it can be shown that

$$
\frac{1}{1-\frac{1}{S_{f}^{*}}}<\frac{1}{1-\frac{1}{S_{f}^{+}(\infty)}} \Rightarrow S_{f}^{*}>S_{f}^{+}(\infty) .
$$

Then the condition (2.123) can be presented in the following form

$$
S_{f}^{n}<S_{f}^{+}(\infty)<S_{f}^{*}
$$

that is always fulfilled because critical asset price for perpetual American calls $S_{f}^{+}(\infty)$ represents an upper bound for the optimal exercise boundary [72].

We have proved that $d^{n}>1$. Moreover, from 2.122) and 2.105) follows that $d^{n}=1+O(k)$. From 2.100 one gets that $a_{1}^{n}>a_{1}>0$, and

$$
a_{2}^{n}=\frac{k \sigma^{2}}{2 h^{2}}-\left(r-q-\frac{\sigma^{2}}{2}\right) \frac{k}{2 h}-\frac{d^{n}-1}{2 h}>0 .
$$

Since all coefficients of the scheme (2.99) are positive, under (2.120) $c_{j+1}^{n+1}-c_{j}^{n+1}=a_{1}^{n}\left(c_{j}^{n}-c_{j-1}^{n}\right)+b\left(c_{j+1}^{n}-c_{j}^{n}\right)+a_{2}^{n}\left(c_{j+2}^{n}-c_{j+1}^{n}\right)+k q S_{f}^{n+1} e^{-j h}\left(1-e^{-h}\right)>0$.

The positivity of the values $c_{j}^{n+1}$ follows from the increasing behaviour in index $j$ and boundary condition 2.101. 
Let us denote

$$
y(z)=1+\frac{\left(\left(b-1+4 a_{2}\right) \frac{q h^{2}}{\sigma^{2}}-k q e^{-h}\right) z+r k}{(2+h) \frac{q h}{\sigma^{2}} z-\frac{2 r h}{\sigma^{2}}},
$$

with negative derivative

$$
\frac{d y}{d z}=-\frac{r q h}{\sigma^{2}} \frac{\left(\left(b-1+4 a_{2}\right) \frac{h^{2}}{\sigma^{2}}-k e^{-h}\right)+(2+h) k}{\left((2+h) \frac{q h}{\sigma^{2}} z-\frac{2 r h}{\sigma^{2}}\right)^{2}}
$$

for small enough values of $h$. Since $y\left(S_{f}^{n}\right)=d^{n}$, and $S_{f}^{n}>S_{f}^{n-1}$, then $d^{n}$ is a decreasing discrete function of $n$. That means that $\left\{S_{f}^{n}\right\}$ has a concave behaviour.

Now the following results have been established:

Theorem 2.3.1. Let $\left\{c_{j}^{n}, S_{f}^{n}\right\}$ be the numerical solution of scheme (2.99), (2.100), (2.101) for the transformed American call option problem (2.93) and let $d^{n}$ be defined by (2.107). Then under conditions (2.112), (2.113), the numerical solution presents the following properties:

1. Increasing monotone concave behaviour and positivity of values $S_{f}^{n}, n=$ $0, \ldots, N$;

2. Non-negativity of the vectors $c^{n}=\left(c_{0}^{n}, \ldots c_{M}^{n}\right), n=0, \ldots, N$;

3. Increasing monotonicity of the vectors $c^{n}$ with respect to space index for each fixed $n=0, \ldots, N$.

As it has been mentioned in Chapter 1, consistency of a numerical scheme with respect to a partial differential equation means that the exact theoretical solution of the PDE approximates well the exact theoretical solution of the difference scheme as the step size discretization tends to zero [30]. In order to study the consistency let us take an arbitrary point $(x, \tau)$ in the domain $(0, \infty) \times(0, T]$ and consider the mesh points $\left(x_{j}, \tau^{n}\right)$ given by (2.14). Let us assume that the function $c(x, \tau)$ admits four times continuous partial derivatives with respect to $x$ and twice continuous partial derivatives with respect to $\tau$ as well as the function $S_{f}(\tau)$ is twice differentiable. Using Taylor's expansion about $\left(x_{j}, \tau^{n}\right)$, the local truncation error takes the following form 


$$
T_{j}^{n}\left(\tilde{c}, \tilde{S}_{f}\right)=O\left(h^{2}\right)+O(k) .
$$

With respect to the additional boundary condition (2.98), let us denote

$$
\begin{aligned}
L_{b c}\left(c, S_{f}\right) & =\frac{\sigma^{2}}{2} \frac{\partial^{2} c}{\partial x^{2}}(0, \tau)-q S_{f}(\tau)+r=0, \\
F_{b c}\left(c^{n}, S_{f}^{n}\right) & =\frac{\sigma^{2}}{2} \frac{c_{-1}^{n}-2 c_{0}^{n}+c_{1}^{n}}{h^{2}}-q S_{f}^{n}+r=0 .
\end{aligned}
$$

Truncation error satisfies $F_{b c}\left(\tilde{c}, \tilde{S}_{f}\right)-L_{b c}\left(\tilde{c}, \tilde{S}_{f}\right)=O\left(h^{2}\right)$. The truncation error for the boundary condition behaves as $h^{2}$. Analogously, truncation error for the boundary condition (2.95) satisfies second order in space because of the central difference approximation 2.101.

Theorem 2.3.2. Assuming that the solution of the PDE problem (2.93)-(2.97) admits two times continuous partial derivative with respect to time and up to order four with respect to space, the numerical solution computed by the scheme (2.99), (2.100) is consistent with the equation (2.93) and boundary conditions (2.95), (2.98) of order two in space and order one in time.

\subsubsection{Numerical examples}

Example 2.3.1. In order to compare computational efficiency of the method and to study the convergence rate, we consider the problem with the parameters [54]:

$$
r=0.03, \quad q=0.03, \quad \sigma=0.4, \quad T=0.5, \quad E=100 .
$$

Table 2.7 shows the comparison of the proposed method with other methods in [54]. Since exact values are not known, the results of the binomial method with large steps (15000) are used for "True Value". FDP stands for the CrankNicolson finite-difference method with projected SOR iteration to impose the free boundary condition. FDE stands for the Crank-Nicolson finite difference method with elimination-back substitution. HW stands for the Han and Wu method (see [54]). FF stands for the proposed explicit finite difference method combined with the front-fixing transformation with stepsizes: FF1 for $h=2 \cdot 10^{-3}$ and $k=2 \cdot 10^{-5}$, FF2 for $h=2 \cdot 10^{-3}$ and $k=6.25 \cdot 10^{-6}$, FF3 for $h=10^{-3}$ and $k=6.25 \cdot 10^{-6}$. The root-mean-square error (RMSE) is used to measure the accuracy of the scheme. The last row presents the CPU-time in seconds for each experiment. 


\section{LINEAR BLACK-SCHOLES MODEL FOR AMERICAN OPTIONS}

\begin{tabular}{|c|c|c|c|c|c|c|c|}
\hline$S$ & True Value & FDP & FDE & HW & FF1 & FF2 & FF3 \\
\hline 40 & 0.002792 & 0.0025 & 0.0025 & 0.0028 & 0.0025 & 0.0027 & 0.0028 \\
\hline 50 & 0.045594 & 0.0457 & 0.0457 & 0.0457 & 0.0451 & 0.0453 & 0.0456 \\
\hline 60 & 0.301387 & 0.3014 & 0.3015 & 0.3015 & 0.3005 & 0.3011 & 0.3015 \\
\hline 70 & 1.145799 & 1.1459 & 1.1461 & 1.1461 & 1.1442 & 1.1451 & 1.1456 \\
\hline 80 & 3.041536 & 3.0414 & 3.0415 & 3.0415 & 3.0401 & 3.0411 & 3.0413 \\
\hline 90 & 6.328677 & 6.3285 & 6.3287 & 6.3287 & 6.3266 & 6.3274 & 6.3284 \\
\hline 100 & 11.108407 & 11.1066 & 11.1068 & 11.1068 & 11.1051 & 11.1072 & 11.1080 \\
\hline 110 & 17.266726 & 17.2664 & 17.2665 & 17.2665 & 17.2632 & 17.2653 & 17.2663 \\
\hline 120 & 24.564972 & 24.5654 & 24.5655 & 24.5655 & 24.5603 & 24.5641 & 24.5648 \\
\hline \multicolumn{2}{|r|}{ RMSE } & $6.4217-4$ & $5.8822-4$ & $5.8012-4$ & $2.4771-3$ & $9.3865-4$ & $2.5088-4$ \\
\hline \multicolumn{2}{|c|}{ CPU-time, sec } & 37.130 & 15.760 & 11.500 & 7.169 & 27.805 & 32.794 \\
\hline
\end{tabular}

Table 2.7: Computational efficiency of various methods in Example 2.3.1.

Results presented in Table 2.7 show the competitiveness of the proposed method.

It was theoretically proved in previous section that the scheme has order of approximation $O\left(h^{2}\right)+O(k)$. To check numerically the order of approximation we use convergence rate.

From the Table 2.7, one obtains $\gamma_{h}\left(2 \cdot 10^{-3}, 10^{-3}\right)=1.9036$, that is close to 2 . Analogously it is found that $\gamma_{k}\left(2 \cdot 10^{-5}, 6.25 \cdot 10^{-6}\right)=0.8343$, that is close to 1 , that proves the second order of approximation in space and the first order in time.

Example 2.3.2. In order to compare the proposed front-fixing method with the fixed domain transformation presented in [109], a problem with the following parameters

$$
r=0.1, \quad q=0.05, \quad \sigma=0.2, \quad T=1, \quad E=10 .
$$

is considered.

The position of the free boundary at $\tau=T$ is $S_{f}(T)=22.3754$ (in [109]) and the proposed method gives $S_{f}(T)=22.375$. In Table 2.8 a comparison of results obtained by the proposed front-fixing method (FF) and by other methods such as fixed domain transformation presented in [109] (FD), trinomial tree (Tree), finite difference approximation (FD) and Analytical Approximation (AA) of BaroneAdesi and Whaley [7] is presented. The optimal stopping boundary motion in time is presented in Figure 2.4. One can see that the qualitative properties are preserved 


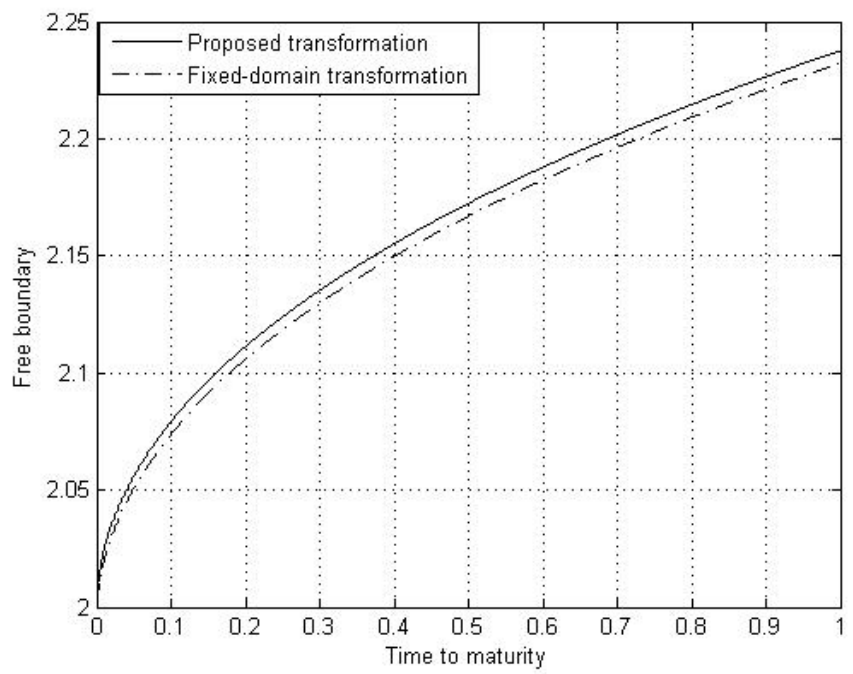

Figure 2.4: Optimal stopping boundary in Example 2.3.2 by using proposed transformation and fixed-domain transformation [109].

with the proposed scheme: the free boundary value is positive, increasing in time with concave behaviour.

Example 2.3.3. Let us consider the problem with parameters

$$
r=0.1, \quad q=0.05, \quad \sigma=0.2, \quad T=1,
$$

to compare explicit and implicit schemes of the proposed method.

In Figure 2.5 we compare explicit and implicit methods with $h=0.01$ and dif-

\begin{tabular}{|c|c|c|c|c|c|}
\hline $\mathrm{S}$ & FD & Treel & FD & AA & FF \\
\hline 15 & 5.15 & 5.15 & 5.49 & 5.23 & 5.16 \\
18 & 8.09 & 8.09 & 8.48 & 8.10 & 8.10 \\
20 & 10.03 & 10.03 & 10.48 & 10.04 & 10.04 \\
21 & 11.01 & 11.01 & 11.48 & 11.02 & 11.02 \\
22.375 & 12.37 & 13.37 & 12.48 & 12.38 & 12.38 \\
\hline
\end{tabular}

Table 2.8: Comparison of the proposed method with other methods for parameters (2.128. 


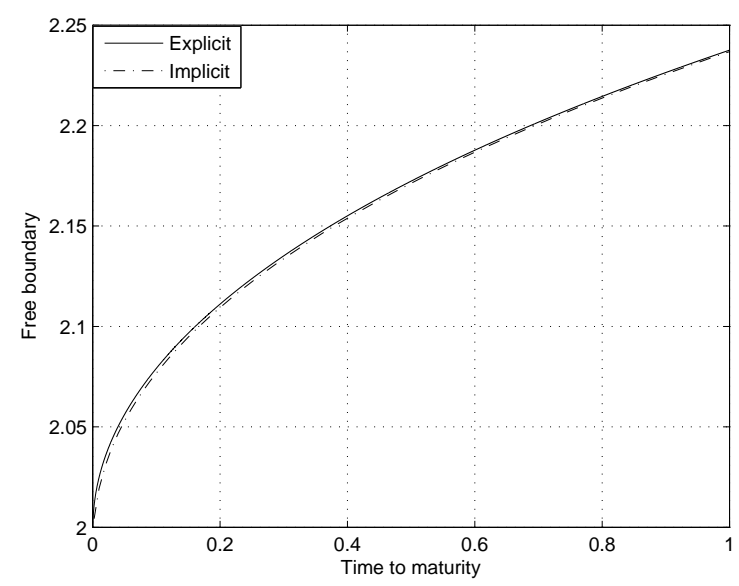

Figure 2.5: Optimal stopping boundary in Example 2.3.3 computed by explicit and implicit methods.

ferent time steps: $k=10^{-4}$ for the explicit method to guarantee condition 2.113 and $k=0.01$ for the implicit method. The result of fully implicit method is presented in Figure 2.6 .

Implicit method is unconditionally stable, that allows to reduce computational time. But, there are additional calculations of the inverse Jacobian matrix on each iteration. The computational time for both methods is presented in Table 2.9. It is shown that for the same step sizes the explicit method is ten times faster than implicit one. In the case of the smaller space steps for the explicit method we have to choose time steps much smaller, but the total computational time is almost ten times less.

The results of this section have been published in [24]. 


\subsection{New efficient front-fixing method for American option pricing}

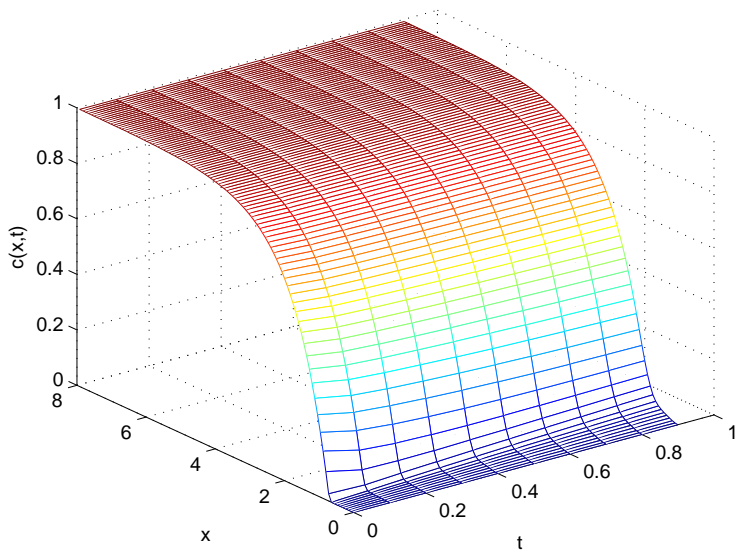

Figure 2.6: The function $c(x, \tau)$ calculated by the proposed fully implicit method.

\begin{tabular}{|c|c|c|c|c|}
\hline Method & $h$ & $k$ & CPU-time, sec. & $S_{f}(T)$ \\
\hline Explicit & $10^{-1}$ & $10^{-2}$ & 0.016 & 2.2283 \\
& $10^{-2}$ & $10^{-4}$ & 3.693 & 2.2375 \\
& $10^{-3}$ & $10^{-6}$ & 28.918 & 2.2376 \\
\hline Implicit & $10^{-1}$ & $10^{-2}$ & 0.179 & 2.2269 \\
& $10^{-2}$ & $10^{-2}$ & 16.029 & 2.2368 \\
& $10^{-3}$ & $10^{-2}$ & 107.435 & 2.2375 \\
\hline
\end{tabular}

Table 2.9: Comparison of the computational time and accuracy for explicit and implicit methods. 



\section{Front-fixing method for some advanced models}

Pricing American option is a problem often studied in the field of computational finance. The well-known Black-Scholes (BS) model [10] provides an easy computable pricing formula of European option in an idealistic market. However, assumptions of BS model that have been summarized in Chapter 1 are not realistic [14], [82]. BS assumptions do not take into account, for instance, transaction costs, investor's preferences, feedback and illiquid markets effects. This has motivated the development of new nonlinear models to take account of various realistic trading environment.

Leland [82] proposed a BS formula with an augmented volatility due to transaction cost. Authors in [4], [110] presented an adjusted volatility which depends on the sign of the gamma of the option to control effectively the hedging risk and transaction cost. These ideas led to a nonlinear BS equation for European options. Barles and Soner [6] proposed a more complicated nonlinear model by assuming that investor's preferences are characterized by an exponential utility function. Later risk adjusted pricing methodology (RAPM) was proposed by Kratka [75] and revisited by Jandačka and Ševčovič in [63]. Note that all the above mentioned nonlinear models are consistent with the original BS equation in the case when the additional parameters are vanishing. 


\section{FRONT-FIXING METHOD FOR SOME ADVANCED MODELS}

Valuation of derivatives uses to be based on the assumption of a stochastic process for the underlying asset and the construction of a dynamic, self-financing hedging portfolio to minimize the uncertainty (risk). Using the absence of arbitrage principle, the initial cost of constructing the portfolio, typically given by a partial differential equation (PDE), is then considered to be the fair value of the derivative, [57].

When the stochastic process for the asset is too simple, assuming constant parameters, like [10] the model does not replicate the market price. This drawback has been overcome with stochastic volatility, jump diffusion and regime switching models. Furthermore, regime switching models are computationally inexpensive compared to stochastic volatility jump diffusion models and have versatile applications in other fields, like electric markets [9], valuation of stock loans [117], forestry valuation [20], natural gas [21] and insurance [55].

In general there is no closed form solution for nonlinear American or European option pricing problem. Therefore numerical methods are usually employed to solve them. For European options numerical methods have been developed by several authors in recent years [28], [41], [94], etc. For American options the main difficulty is the existence of the unknown optimal stopping boundary. One way to overcome this difficulty is to present it as a nonlinear complementarity problem (NCP) arising from the discretisation of the free boundary problem. In [56] and [83] the penalty approach is proposed to solve the NCP by approximating it using an algebraic system of nonlinear equations containing a power penalty term.

A common alternative approach to NCP that is able to remember the free boundary while solving the problem is the so-called front-fixing method [33], based on the transformation of the original equation into a new one defined on a fixed domain. The unknown free boundary is calculated as an additional unknown function involved in the PDE problem. Although free boundary problems originated in physics, this technique has been used in computational finance since 1997 [112]. In Chapter 2 some front-fixing transformations and numerical schemes have been proposed for linear BS American option pricing.

In this chapter new transformations in the framework of a front-fixing method are proposed for nonlinear Barles and Soner's model, RAPM and regime switching model. For nonlinear models the transformation is used to replace the free boundary by the time-dependent known boundary. Multi-variable front-fixing transformation is used for regime switching model since optimal stopping boundaries for various regimes differ each other. 


\subsection{Nonlinear Black-Scholes models}

For the case of American options with constant volatility various front-fixing transformations have been studied in [24], [77], [90], [107]. Ševčovič proposed a fixed domain transformation for nonlinear American option pricing problem [108]. Further, this method was studied in some recent papers (see [2]). Since the transformed equation contains a strong convective term the operator splitting method is used to overcome numerical difficulties. Moreover, in order to close up the system of equations that determines the value of a new function an additional equation for the free boundary position is required.

We propose efficient front-fixing method for nonlinear Black-Scholes equation. Under the transformation the free boundary is replaced by a time-dependent known boundary. In the resulting equation there is no reaction term and the convection term is simplified in a such way that the operator splitting technique is not required. This ensured a single numerical scheme is suitable for the entire equation. The connection between the transformed boundary conditions with the transformed option price and the free boundary does not require additional information.

The proposed formulation of the nonlinear problem allows the use of a versatile numerical treatment. In this chapter an explicit Euler and alternating direction explicit (ADE) method [38], [92] together with implicit methods are studied. Dealing with implicit methods one has to solve nonlinear system. In this section Newton's method with suitable modifications to improve its efficiency and in saving computational cost [78] are examined.

\subsubsection{Moving domain transformation}

The transformation of the free boundary American option pricing problem into another nonlinear PDE problem, such that the free boundary is written in terms of another variable with a known moving boundary, is presented.

With the previous notation, nonlinear American call option pricing models may be formulated as the free boundary PDE problem

$$
\frac{\partial C}{\partial \tau}=\frac{\tilde{\sigma}^{2}}{2} S^{2} \frac{\partial^{2} C}{\partial S^{2}}+(r-q) S \frac{\partial C}{\partial S}-r C, \quad 0 \leq S<B(\tau), 0<\tau \leq T,
$$

where the adjusted volatility function is given by

$$
\tilde{\sigma}^{2}=\tilde{\sigma}^{2}\left(\tau, S, C_{S S}\right)
$$




\section{FRONT-FIXING METHOD FOR SOME ADVANCED MODELS}

The boundary and initial conditions for an American call option problem are (see [111])

$$
\begin{aligned}
C(S, 0) & =\max (S-E, 0), \\
\frac{\partial C}{\partial S}(B(\tau), \tau) & =1, \\
C(B(\tau), \tau) & =B(\tau)-E, \\
C(0, \tau) & =0, \\
B(0) & =\max \left(\frac{r}{q} E, E\right) .
\end{aligned}
$$

It is well known that if there is no dividend payment $(q=0)$, then the optimal strategy is to exercise option at the maturity (see [111], [59]). In that case the American call becomes an European call. Due to this reason $q>0$ [59] is used in the problem defined in (3.1)-3.7).

In the following study, two nonlinear models with different adjusted volatility functions 3.2 are considered. First the strong nonlinear RAPM model, where $\tilde{\sigma}^{2}$ is a cubic root function. Second the widely used Barles and Soner's model in which the adjusted volatility function is obtained through the solution of an ordinary differential equation.

Under the RAPM model the volatility $\sigma$ is a function of the asset price $(S)$ and the second derivative of the option price $\left(\frac{\partial^{2} C}{\partial S^{2}}\right)$, i.e.

$$
\tilde{\sigma}^{2}=\sigma_{0}^{2}\left(1+\mu\left(S \frac{\partial^{2} C}{\partial S^{2}}\right)^{\frac{1}{3}}\right)
$$

where $\sigma_{0}^{2}$ is a constant historical volatility of the asset and $\mu$ is a non-negative constant.

Barles and Soner introduced a nonlinear Black-Scholes equation with an adjusted volatility [6] which is a function of the second derivative of the price itself, i.e.

$$
\tilde{\sigma}^{2}=\sigma_{0}^{2}\left(1+\Psi\left(e^{r \tau} a^{2} S^{2} C_{S S}\right)\right),
$$

where $a=\mu \sqrt{\gamma N}, \gamma$ is the risk aversion factor and $N$ is the number of options to be sold. The function $\Psi$ is the solution of the nonlinear singular initial value problem

$$
\Psi^{\prime}(A)=\frac{\Psi(A)+1}{2 \sqrt{A \Psi(A)}-A}, \quad A \neq 0, \quad \Psi(0)=0
$$


From the Theorem 1.1 of [31] it is known that $\Psi(A)$ is an increasing function mapping the real line onto the interval $]-1,+\infty]$ and is implicitly defined by

$$
\begin{aligned}
& A=\left(-\frac{\operatorname{arcsinh} \sqrt{\Psi}}{\sqrt{\Psi+1}}+\sqrt{\Psi}\right)^{2}, \quad \Psi>0 ; \\
& A=-\left(\frac{\arcsin \sqrt{-\Psi}}{\sqrt{\Psi+1}}+\sqrt{-\Psi}\right)^{2}, \quad-1<\Psi<0 .
\end{aligned}
$$

The case for Barles and Soner's model is a slightly complicated one in terms of numerical implementation. By using numerical examples it is able to demonstrate that the proposed method may be used to handle other models with nonconstant volatility.

Taking advantages of Landau transformation [79] with modifications in the exponential factors like those described in [28], it is possible to remove the reaction term and partially the convection term by using the transformation given below.

$$
x=e^{(r-q) \tau} \frac{S}{B(\tau)}, \quad V(x, \tau)=\frac{e^{r \tau}}{E} C(S, \tau), \quad S_{f}(\tau)=\frac{B(\tau)}{E} .
$$

Using transformation (3.11) the equation (3.1) takes the form

$$
V_{\tau}=\frac{\sigma^{2}}{2} x^{2} V_{x x}+\frac{S_{f}^{\prime}}{S_{f}} x V_{x}, \quad 0 \leq x<e^{(r-q) \tau}, \quad 0<\tau \leq T
$$

where

$$
\sigma^{2}=\sigma^{2}\left(\tau, x, V_{x x}\right)=\tilde{\sigma}^{2}\left(\tau, S, C_{S S}\right),
$$

with new initial and boundary conditions

$$
\begin{aligned}
S_{f}(0) & =\max \left(\frac{r}{q}, 1\right) \\
V(x, 0) & =\max \left(x S_{f}(0)-1,0\right), \\
V(0, \tau) & =0 \\
V\left(e^{(r-q) \tau}, \tau\right. & =e^{r \tau}\left(S_{f}(\tau)-1\right), \\
V_{x}\left(e^{(r-q) \tau}, \tau\right) & =e^{q \tau} S_{f}(\tau) .
\end{aligned}
$$

Note that the transformation described in (3.11) transformed the original free boundary value problem to a known moving boundary problem. In the case $r>q$ the computational domain increases with respect to time, otherwise it decreases.

In the problem (3.12) - 3.17) there are two sources of nonlinearity. First, the additional unknown function (free boundary) in the equation 3.12 . The method 


\section{FRONT-FIXING METHOD FOR SOME ADVANCED MODELS}

to handle this problem relies on the choice of the finite difference method and is explained further. Second, the volatility $\sigma$ is nonlinear. With the moving domain transformation (3.11) argument of the function $\sigma$ in RAPM model changes and is given below.

$$
\sigma^{2}=\sigma_{0}^{2}\left(1+\mu\left(x V_{x x} \frac{e^{-q \tau}}{S_{f}(\tau)}\right)^{\frac{1}{3}}\right) .
$$

For Barles and Soner's model volatility $\sigma^{2}$ is transformed to

$$
\sigma^{2}=\sigma_{0}^{2}\left(1+\Psi\left(a^{2} E x^{2} V_{x x}\right)\right) .
$$

\subsubsection{Preliminary computational algorithms}

This section begins with an algorithm computing the implicitly adjusted volatility function $\Psi$ given by Barles and Soner's model. It follows with a description of three finite difference methods of solving the transformed problem described in equation 3.12). These finite difference methods include an explicit Euler method, an alternating direction explicit method and an implicit method.

Note that the numerical evaluation of the adjusted volatility function for the RAPM model is straight forward as an explicit function of the volatility is defined. In the case of Barles and Soner's model the volatility function is given in terms of the solution $\Psi$ of the ODE (3.8). It is well known that MATLAB built-in solver for ODE [2], [108] may be used, or in some other cases simply take $\Psi(A)=$ $A$. One way to avoid the additional errors due to the numerical solution of the ODE is to make use of the implicit solution (3.9)-3.10), given in [31], through an interpolation procedure as proposed in the numerical algorithm below.

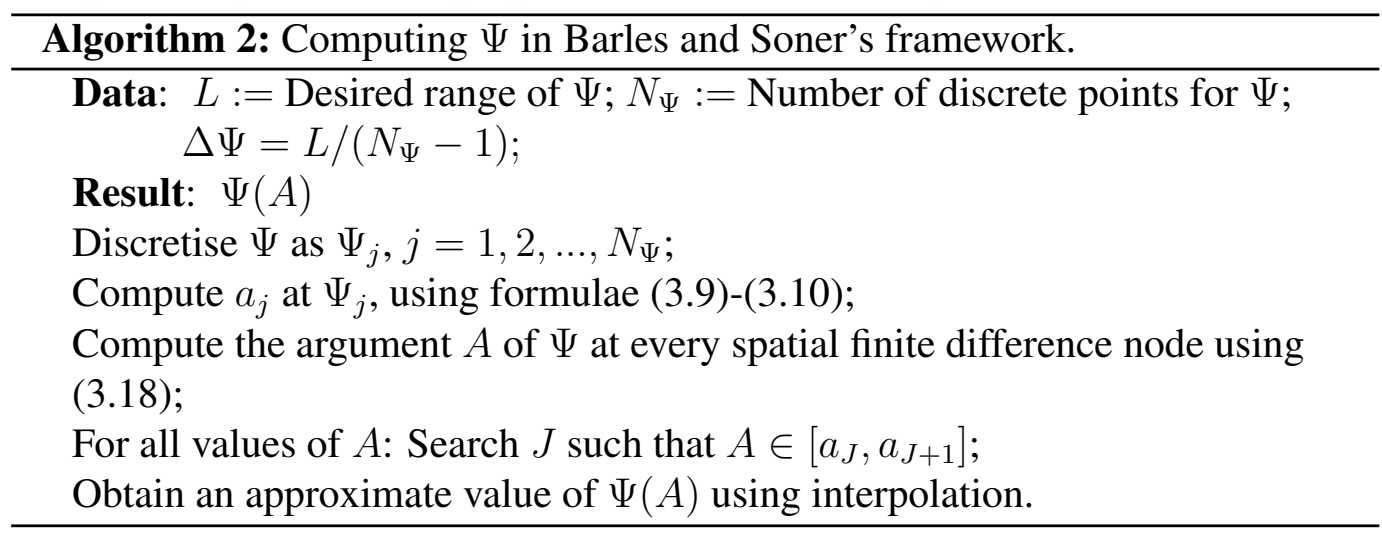

There exists many search algorithms in literature. It is very important to choose an appropriate one. The simplest and widely used one is the linear search. It is 


\begin{tabular}{|l|c|c|}
\hline Number of calls & $10^{3}$ & $10^{4}$ \\
\hline Linear search & 5.022 & 47.600 \\
Binary search & 0.062 & 0.707 \\
\hline
\end{tabular}

Table 3.1: CPU-time (sec) of linear and binary search algorithms.

a method for finding a particular value (key) in an array that checks each element in sequence until the desired element is found or the list is exhausted [74]. The cost of the worst case is proportional to the number of elements in the array. Since function $\Psi(A)$ is increasing, the list of search is also an increasing sequence, i.e. it is sorted. Therefore binary search can be used. In each step, the algorithm compares the key value with the middle element of the list. If the values match, then a matching element has been found. Otherwise, if the search key is less than the middle element, then the algorithm repeats its action on the sub-array to the left of the middle element or, if the search key is greater, on the sub-array to the right. Binary search takes logarithmic time (see [74], p. 414).

For the search of just one element (i.e. calculate one value of $\sigma$ ), both algorithms work with similar speed. The difference becomes noticeable when the procedure repeats several times. Results of the tests are presented in the Table 3.1 Number of calls there means the number of repetition of the procedure.

In the finite difference methods described below for the solutions of (3.12)(3.17), the temporal axis takes a uniform partition with the time step $k=\frac{T}{N}$. Each time level is denoted as $\tau^{n}=n k, 0 \leq n \leq N$. Let the right boundary of the domain be denoted as $x_{\max }^{n}=e^{(r-q) \tau^{n}}$. The spatial step size $h_{n}$ and grid point $x_{j}^{n}$ at time level $\tau^{n}$ are defined as

$$
h_{n}=\frac{e^{(r-q) n k}}{M}, \quad x_{j}^{n}=j h_{n}, 0 \leq j \leq M,
$$

where $M$ is the number of spatial grid points.

Denote the approximate value of the solution $V(x, \tau)$ at the point $x_{j}^{n}$ and time $\tau^{n}$ as $u_{j}^{n} \approx V\left(x_{j}^{n}, \tau^{n}\right)$ and the approximate value of the free boundary as $S_{f}^{n} \approx$ $S_{f}\left(\tau^{n}\right)$. As the problem itself has a moving boundary which means that the spatial finite difference mesh would have to be rearranged at every time level. A typical spatial mesh with grid points $\left(x_{j}^{n}, \tau^{n}\right)$ at time $\tau=n k$ does not remain as a grid point at time $\tau=(n+1) k$. Figure 3.1 illustrates the nodal points $\left(x_{j}^{n}, \tau^{n}\right)$ as black dots and those corresponding nodes as white dots and the spatial finite difference mesh remains unchanged, and the corresponding moveable nodes in black dots 
at the next time level. The pair $\left(x_{j}^{n}, \tau^{n+1}\right)$ is known as a non-grid point for this purpose.

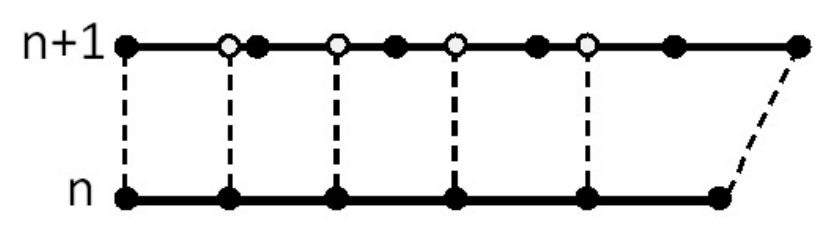

Figure 3.1: Moving grid.

\subsubsection{Explicit Schemes}

Let the approximation at the non-grid point $\left(x_{j}^{n}, \tau^{n+1}\right)$ be denoted as $\tilde{u}_{j}^{n+1} \approx$ $V\left(x_{j}^{n}, \tau^{n+1}\right)$. Once the approximations $\left\{\tilde{u}_{j}^{n+1}\right\}$ are computed $\left\{u_{j}^{n+1}\right\}$ may be obtained by using a Lagrange interpolation from the resulting data $\left\{\tilde{u}_{j}^{n+1}\right\}$.

For the numerical solution of the problem (3.12)-(3.17) an explicit finite difference scheme based on a central difference scheme for the spatial derivatives and a forward difference scheme, for the temporal derivative is constructed as follows

$$
\frac{\tilde{u}_{j}^{n+1}-u_{j}^{n}}{k}=\frac{\sigma_{j, n}^{2}}{2}\left(x_{j}^{n}\right)^{2} \frac{u_{j-1}^{n}-2 u_{j}^{n}+u_{j+1}^{n}}{h_{n}^{2}}+\frac{S_{f}^{n+1}-S_{f}^{n}}{k S_{f}^{n}} x_{j}^{n} \frac{u_{j+1}^{n}-u_{j-1}^{n}}{2 h_{n}},
$$

where $\sigma_{j, n}^{2}=\sigma\left(x_{j}^{n}, \tau^{n}\right)$ is computed by using either the RAPM or the Barles and Soner's model. For the RAPM model $\sigma^{2}$ is calculated as follows

$$
\sigma_{j, n}^{2}=\sigma_{0}^{2}\left(1+\mu\left(x_{j}^{n} \frac{e^{-q n k}}{S_{f}^{n}} \frac{u_{j-1}^{n}-2 u_{j}^{n}+u_{j+1}^{n}}{h_{n}^{2}}\right)\right) .
$$

In the case of Barles and Soner's model

$$
\sigma_{j, n}^{2}=\sigma_{0}^{2}\left(1+\Psi\left(E a^{2}\left(x_{j}^{n}\right)^{2} \frac{u_{j-1}^{n}-2 u_{j}^{n}+u_{j+1}^{n}}{h_{n}^{2}}\right)\right) .
$$

From the boundary conditions 3.16, 3.17) one obtains

$$
\begin{gathered}
u_{0}^{n}=0, \quad u_{M}^{n}=e^{r \tau^{n}}\left(S_{f}^{n}-1\right), \\
\frac{u_{M}^{n}-u_{M-1}^{n}}{h_{n}}=e^{q \tau^{n}} S_{f}^{n} \Rightarrow u_{M-1}^{n}=e^{q \tau^{n}} S_{f}^{n}\left(e^{(r-q) \tau^{n}}-h^{n}\right)-e^{r \tau^{n}} .
\end{gathered}
$$


The initial conditions are discretised as follows

$$
S_{f}^{0}=\max \left(\frac{r}{q}, 1\right), \quad u_{j}^{0}=\max \left(S_{f}^{0} x_{j}^{0}-1,0\right), j=0, \ldots, M .
$$

Using the scheme $(3.20)$ for $j=M-1$ and equation $(3.23)$ at the $(n+1)$-th time level, the expression for $S_{f}^{n+1}$ takes the form

$$
\begin{aligned}
& S_{f}^{n+1}=\varrho\left(u^{n}, S_{f}^{n}\right) \\
& =\frac{u_{M-1}^{n}+\frac{\sigma^{2}(M-1, n) k}{2 h_{n}^{2}}\left(x_{M-1}^{n}\right)^{2}\left(u_{M-2}^{n}-2 u_{M-1}^{n}+u_{M}^{n}\right)-x_{M-1}^{n} \frac{u_{M}^{n}-u_{M-2}^{n}}{2 h_{n}}+e^{r \tau^{n}}}{e^{q \tau^{n}}\left(e^{(r-q) \tau^{n}}-h_{n+1}\right)-x_{M-1}^{n} \frac{u_{M}^{n}-u_{M-2}^{n}}{2 h_{n} S_{f}^{n}}} .
\end{aligned}
$$

Assembling all these ideas leads to the following algorithm.

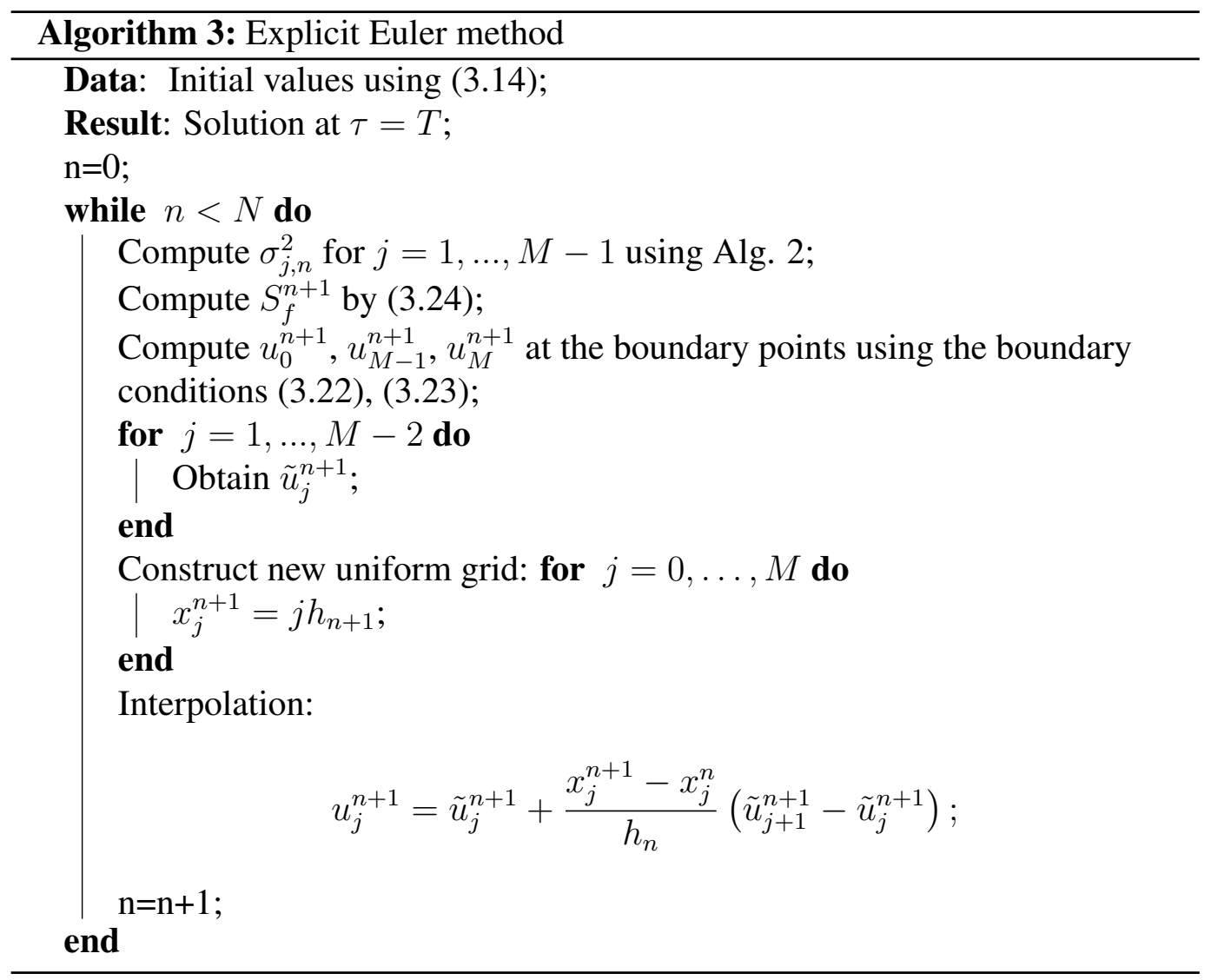

Remark 1. Linear interpolation is used in order to preserve the second order accuracy of the approximations of the spatial derivatives. 


\section{FRONT-FIXING METHOD FOR SOME ADVANCED MODELS}

Remark 2. With the interpolation one needs to guarantee that new grid point $x_{j}^{n+1} \in\left[x_{j}^{n}, x_{j+1}^{n}\right)$. In the case $r>q$ if

$$
j h_{n} \leq j h_{n+1}<(j+1) h_{n},
$$

one has from the definition 3.19,

$$
0 \leq j\left(e^{(r-q) k}-1\right)<1, \quad \forall j .
$$

This inequality is guaranteed if

$$
M\left(e^{(r-q) k}-1\right)<1
$$

which occurs if $k$ satisfies

$$
k<\frac{\ln \left(1+h_{0}\right)}{r-q} .
$$

In the case $r<q$ domain is decreasing and $k<\frac{\ln \left(1+h_{0}\right)}{q-r}$ is a sufficient condition for $x_{j}^{n} \in\left[x_{j}^{n+1}, x_{j+1}^{n+1}\right)$. In the case when $r=q$ the moving boundary is fixed with $x_{M}^{n}=1$ for all $n$ and the interpolation is not necessary.

An Alternating Direction Explicit method combines the advantages of simplicity of an explicit method and the unconditional stability of implicit scheme for the linear case (see [84]). The numerical solution is calculated as the average of two solutions using explicit scheme known as the right direction solution $\left\{R_{j}^{n}\right\}$ and the left direction solution $\left\{L_{j}^{n}\right\}$. The algorithm of the ADE method for the moving domain problem 3.12)-(3.17) is given as follows.

\subsubsection{Implicit numerical methods}

Both implicit and explicit numerical methods have advantages and disadvantages (see introduction of [29]). In previous subsections two explicit numerical methods are discussed and in this subsection implicit schemes are discussed. The discretisation of the nonlinear equation described in 3.12 leads to a system of nonlinear equations. The most popular and widely used method for solving nonlinear systems is the so-called Newton's method. This method is iterative and requires to calculate Jacobian of the nonlinear system every iteration which is time consuming. There exists various modifications of the method [78].

A fully implicit scheme for the equation 3.12 using the same notation as in (3.21) takes the form 


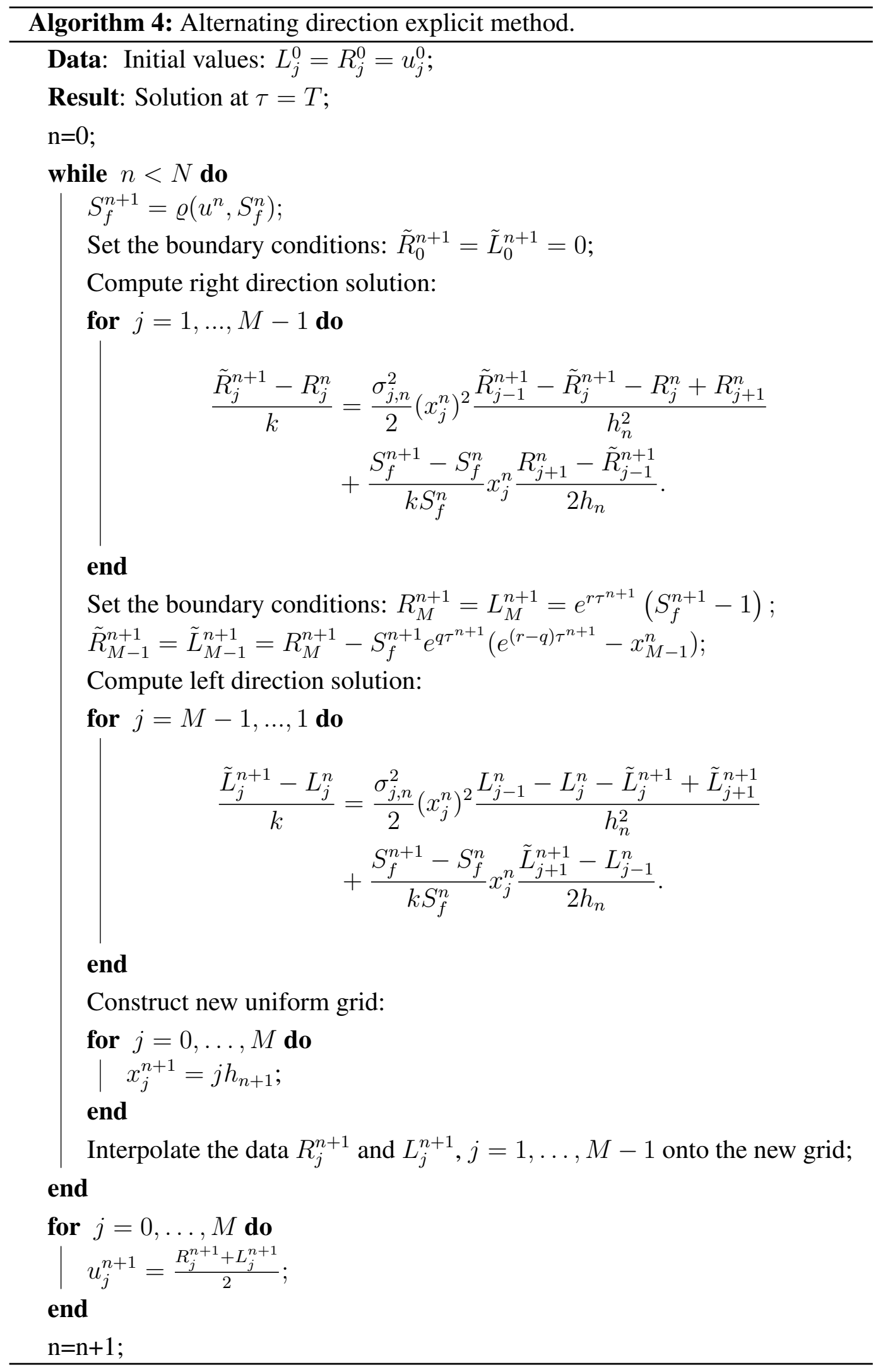




$$
\frac{\tilde{u}_{j}^{n+1}-u_{j}^{n}}{k}=\frac{\sigma_{j, n+1}^{2}}{2}\left(x_{j}^{n}\right)^{2} \frac{\tilde{u}_{j-1}^{n+1}-2 \tilde{u}_{j}^{n+1}+\tilde{u}_{j+1}^{n+1}}{h_{n}^{2}}+\frac{S_{f}^{n+1}-S_{f}^{n}}{k S_{f}^{n+1}} x_{j}^{n} \frac{\tilde{u}_{j+1}^{n+1}-\tilde{u}_{j-1}^{n+1}}{2 h_{n}}
$$

for $j=1, \ldots, M-2$. Since the left boundary is fixed at $x_{0}^{n}=0$, and the right boundary is given by $x_{M}^{n}=e^{(r-q) \tau^{n}}$, the last three points are non-equidistant. Let $\tilde{h}_{n}=x_{M}^{n+1}-x_{M-1}^{n}$. Taylor's series expansion is used to obtain a discretization of the second derivative on the non-uniform grid:

$$
V_{x x}\left(x_{M-1}^{n}, \tau^{n+1}\right) \approx 2\left(\frac{\tilde{u}_{M-2}^{n+1}}{h_{n}\left(h_{n}+\tilde{h}_{n}\right)}+\frac{u_{M}^{n+1}}{\tilde{h}_{n}\left(h_{n}+\tilde{h}_{n}\right)}-\frac{\tilde{u}_{M-1}^{n+1}}{h_{n} \tilde{h}_{n}}\right) .
$$

Using (3.26) and a central difference for the first derivative, the implicit scheme (3.25) for $j=M-1$ takes the following form:

$$
\begin{aligned}
\frac{\tilde{u}_{M-1}^{n+1}-u_{M-1}^{n}}{k} & =\sigma^{2}(M-1, n+1)\left(x_{M-1}^{n}\right)^{2}\left(\frac{\tilde{u}_{M-2}^{n+1}}{h_{n}\left(h_{n}+\tilde{h}_{n}\right)}+\frac{u_{M}^{n+1}}{\tilde{h}_{n}\left(h_{n}+\tilde{h}_{n}\right)}-\frac{\tilde{u}_{M-1}^{n+1}}{h_{n} \tilde{h}_{n}}\right) \\
& +\frac{S_{f}^{n+1}-S_{f}^{n}}{k S_{f}^{n+1}} x_{M-1}^{n} \frac{u_{M}^{n+1}-\tilde{u}_{M-2}^{n+1}}{2 h_{n}} .
\end{aligned}
$$

Boundary conditions are discretised as follows

$$
u_{M}^{n+1}=e^{r \tau^{n+1}}\left(S_{f}^{n+1}-1\right), \quad \frac{u_{M}^{n+1}-\tilde{u}_{M-1}^{n+1}}{\tilde{h}_{n}}=e^{q \tau^{n+1}} S_{f}^{n+1} .
$$

Define the following coefficients

$$
\begin{aligned}
a_{-1}(j, n+1)= & -\frac{k}{h_{n}^{2}}\left(x_{j}^{n}\right)^{2} \frac{\sigma_{j, n+1}^{2}}{2}+\left(1-\frac{S_{f}^{n}}{S_{f}^{n+1}}\right) \frac{x_{j}^{n}}{2 h_{n}}, \\
a_{0}(j, n+1)= & 1+\frac{k}{h_{n}^{2}}\left(x_{j}^{n}\right)^{2} \sigma_{j, n+1}^{2}, \\
a_{+1}(j, n+1)= & -\frac{k}{h_{n}^{2}}\left(x_{j}^{n}\right)^{2} \frac{\sigma_{j, n+1}^{2}}{2}-\left(1-\frac{S_{f}^{n}}{S_{f}^{n+1}}\right) \frac{x_{j}^{n}}{2 h_{n}}, \\
\tilde{a}_{-1}(n+1)= & -\frac{k}{h_{n}\left(h_{n}+\tilde{h}_{n}\right)}\left(x_{M-1}^{n}\right)^{2} \sigma^{2}(M-1, n+1) \\
& +\left(1-\frac{S_{f}^{n}}{S_{f}^{n+1}}\right) \frac{x_{M-1}^{n}}{h_{n}+\tilde{h}},
\end{aligned}
$$




$$
\begin{aligned}
\tilde{a}_{0}(n+1)= & 1+\frac{k}{h_{n} \tilde{h}_{n}}\left(x_{M-1}^{n}\right)^{2} \sigma^{2}(M-1, n+1), \\
\tilde{a}_{-1}(n+1)= & -\frac{k}{h_{n}\left(h_{n}+\tilde{h}_{n}\right)}\left(x_{M-1}^{n}\right)^{2} \sigma^{2}(M-1, n+1) \\
& -\left(1-\frac{S_{f}^{n}}{S_{f}^{n+1}}\right) \frac{x_{M-1}^{n}}{h_{n}+\tilde{h}} .
\end{aligned}
$$

The fully implicit scheme can be expressed in the matrix form

$$
A_{n+1} U_{n+1}=B_{n}
$$

where

$$
\begin{gathered}
A_{n+1}=\left(\begin{array}{cccccc}
a_{0}(1, n+1) & a_{+1}(1, n+1) & 0 & 0 & \cdots & 0 \\
a_{-1}(2, n+1) & a_{0}(2, n+1) & a_{+1}(2, n+1) & 0 & \cdots & 0 \\
0 & \ddots & \ddots & \ddots & \ddots & \vdots \\
0 & \cdots & \tilde{a}_{-1}(n+1) & \tilde{a}_{0}(n+1) & \tilde{a}_{+1}(n+1) & 0 \\
0 & \cdots & 0 & -1 & 1 & -e^{q \tau^{n+1}} \tilde{h}_{n} \\
0 & \cdots & 0 & 0 & -1 & e^{r \tau^{n+1}}
\end{array}\right) \\
U_{n+1}=\left(\begin{array}{c}
\tilde{u}_{1}^{n+1} \\
\tilde{u}_{1}^{n+1} \\
\vdots \\
\tilde{u}_{M-1}^{n+1} \\
u_{M}^{n+1} \\
S_{f}^{n+1}
\end{array}\right), \\
B_{n}=\left(\begin{array}{c}
u_{1}^{n} \\
u_{2}^{n} \\
\vdots \\
u_{M-1}^{n} \\
0 \\
e^{r \tau^{n+1}}
\end{array}\right) .
\end{gathered}
$$

Newton's method is applied to solve the nonlinear system (3.27). At each time level an initial guess is required for the iterative process in the Newton's method and may be chosen as the approximate solution at the previous time level. The stopping criterion is chosen to be the norm of the increment becomes smaller than 
the tolerance $\epsilon$.

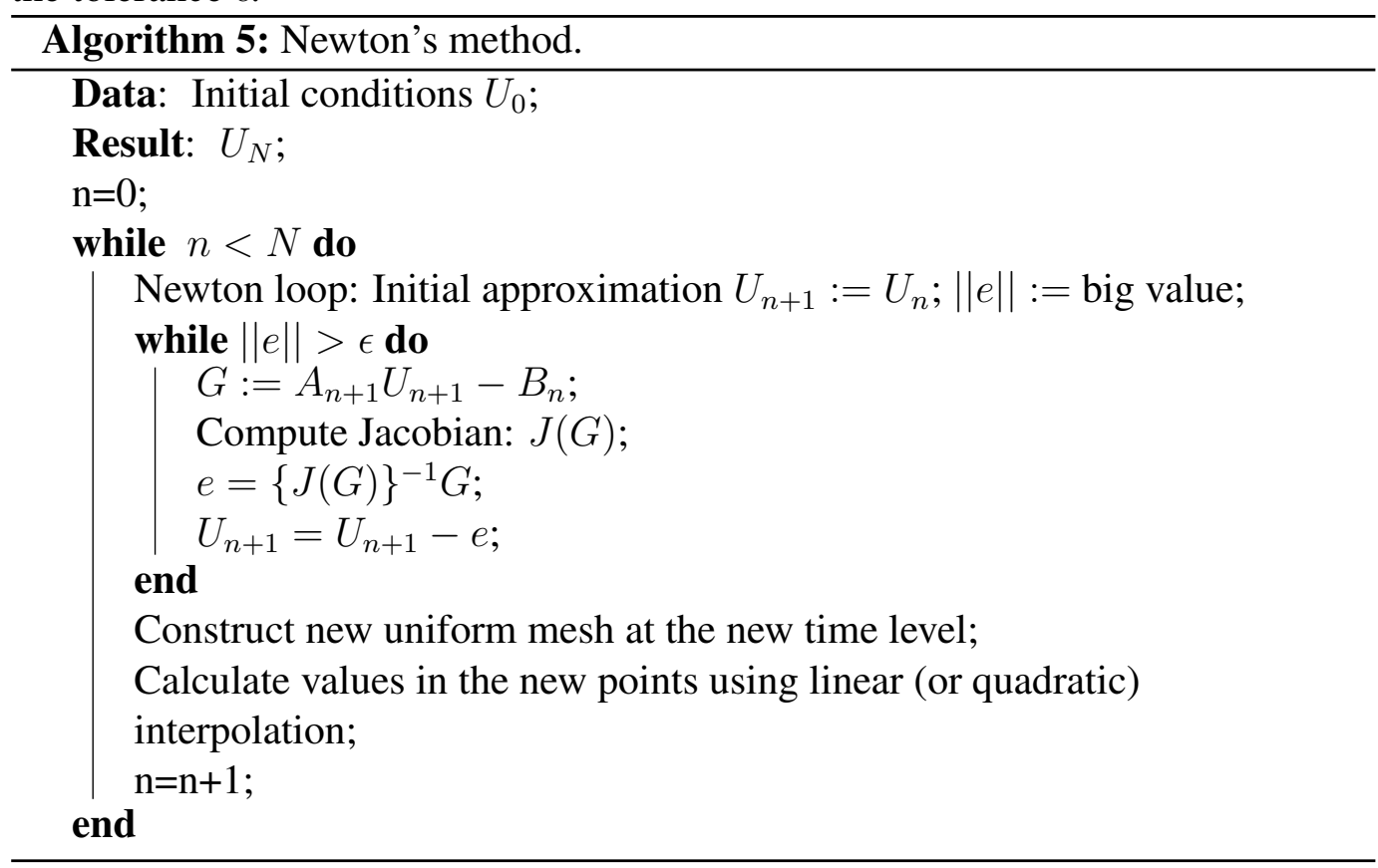

There are many modifications of Newton's method mainly to improve the efficiency and robustness of the method. One type of modification aims to avoid the computations of Jacobian every iteration in order to reduce the total computational time. These methods are collectively known as Newton-like methods. For instance, the main idea of Broyden's method is to calculate an approximate Jacobian iteratively using simple matrix vector multiplications as given below.

$$
J_{k}=J_{k-1}+\frac{\Delta G_{k}-J_{k-1} \Delta u_{k}}{\left\|\Delta u_{k}\right\|^{2}} \Delta u_{k}^{T}
$$

where $k$ is the number of current Newton's iteration, $\Delta G_{k}=G_{k}-G_{k-1}, \Delta u_{k}=$ $u_{k}-u_{k-1}$. The initial value $J_{0}$ has to be calculated by a standard procedure to avoid instability. Since $\Delta G_{k}-J_{k-1} \Delta u_{k} \approx G_{k+1}$, 3.28) can be presented in the following form

$$
J_{k}=J_{k-1}+\frac{G_{k+1}}{\left\|\Delta u_{k}\right\|^{2}} \Delta u_{k}^{T}
$$

Unfortunately, if the Jacobian has a given sparsity structure, as it occurs in the present study, Broyden's approximation breaks the structure and introduces nonzero values to those zero components. Schubert's method [88] is widely used for sparse matrices because it preserves the sparsity of the Jacobian. Although it has 
good properties, it is sensitive to the problem under consideration and size of the matrix. Indeed, our problem is not well conditioned for the Schubert's methods as it is shown in Table 3.5. Therefore, a modification of the method is proposed in order to overcome these computational difficulties. Instead of taking the squared norm in denominator power one was used in all the tests. This modification is denoted as "Schubert-1" method. Numerical tests show that it ensures the convergence of Schubert's algorithm.

In order to overcome the drawback of Broyden's method all of the matrix elements outside the tridiagonal band were "frozen" at zeros in the numerical tests. This modification preserves the structure of the matrix in the same way as Schubert's method does. The proposed modification is known as "frozen-Broyden":

$$
J_{k}(i, j)=\left\{\begin{array}{l}
J_{k}(i, j), \quad j-1 \leq i \leq j+1, \\
0, \quad \text { otherwise }
\end{array}\right.
$$

Spectral analysis confirms quality of the proposed methods by the numerical examples provided in the next Section.

\subsubsection{Numerical examples}

This section is devoted to several numerical tests and a comparison of the explicit and implicit methods as described above. Convergence rate and computational costs for the numerical solution of Barles and Soner's model for American options are presented.

Example 3.1.1. An American call option pricing problem in the transformed form (3.12)-3.17) with the parameters:

$$
r=0.1, q=0.05, T=1, \sigma_{0}=0.2, E=10
$$

is tested.

Barles and Soner's model with $a=0.05$ was chosen in the test. In this example the numerical convergence rate in terms of root mean square error (RMSE) (see [85], p. 385) of the proposed methods are presented. The RMSE is computed by formula 1.20 , with $u^{*}\left(x_{i}, T\right)$ is a "true value" of function $V\left(x_{i}, T\right)$ and $u_{h}\left(x_{i}, T\right)$ is calculated value in the point $\left(x_{i}, \tau^{N}\right)$. Here the "true value" is understood as the numerical solution on a refined grid with step sizes $h=5 \cdot 10^{-3}$ and $k=10^{-5}$. 


\section{FRONT-FIXING METHOD FOR SOME ADVANCED MODELS}

\begin{tabular}{|l|cccc|}
\hline$h_{0}$ & 0.08 & 0.04 & 0.02 & 0.01 \\
\hline & \multicolumn{5}{|c|}{ Explicit method } \\
RMSE & 0.04984 & 0.02629 & 0.01232 & 0.00464 \\
CPU-time, s & 15.810 & 27.566 & 51.476 & 99.434 \\
\hline & \multicolumn{5}{|c|}{ ADE method } \\
RMSE & 0.16816 & 0.08172 & 0.02099 & 0.00620 \\
CPU-time, s & 15.129 & 27.776 & 53.865 & 104.247 \\
\hline & \multicolumn{5}{|c|}{ Implicit method } \\
RMSE & 0.04984 & 0.02355 & 0.00958 & 0.00445 \\
CPU-time, s & 34.099 & 60.030 & 112.728 & 257.880 \\
\hline & \multicolumn{5}{|c|}{ Newton-like method } \\
RMSE & 0.11376 & 0.06026 & 0.01389 & 0.00471 \\
CPU-time, s & 33.869 & 58.141 & 107.561 & 315.505 \\
\hline
\end{tabular}

Table 3.2: RMSE with respect to CPU-time for different $h_{0}$ and fixed $k=0.0001$.

Then the spatial convergence rate of the approximate solutions are calculated for different step sizes $h$ at a fixed time step $k$ by using formula 1.21 .

In Table 3.2 the results and comparison are presented. The time step is fixed at $k=0.0001$ to guarantee stability of all numerical solutions. For implicit method the tolerance was chosen as $\epsilon=10^{-4}$.

From Table 3.2, taking the mean value of all combinations of $h_{1}$ and $h_{2}$, one obtains

$$
\gamma_{\text {expl }}=1.512, \quad \gamma_{i m p l}=1.191, \quad \gamma_{A D E}=1.734, \quad \gamma_{N L}=1.713
$$

An analogous formula to 1.21 can be used for convergence rate in time. RMSE and computational time for a fixed spatial step are presented in Table 3.3 .

Using data from Table 3.3 , the convergence rate in time can be calculated as follows

$$
\gamma_{\text {expl }}=0.627 \gamma_{i m p l}=0.733, \quad \gamma_{A D E}=1.789, \quad \gamma_{N L}=0.691
$$

Note that the main part of the computational time is pertained for the calculation of $\Psi(A)$. For the implicit methods it has to be calculated on each iteration of Newton's method. Thus, their computational costs may be noticeably reduced by choosing another model. 


\begin{tabular}{|l|cccc|}
\hline$k$ & 0.001 & 0.0005 & 0.0002 & 0.0001 \\
\hline & \multicolumn{4}{|c|}{ Explicit method } \\
RMSE & 0.01713 & 0.01373 & 0.00675 & 0.00464 \\
CPU-time, s & 11.470 & 21.361 & 50.598 & 99.434 \\
\hline & \multicolumn{4}{|c|}{ ADE method } \\
RMSE & 0.38763 & 0.08152 & 0.01839 & 0.00620 \\
CPU-time, s & 10.829 & 21.199 & 52.684 & 104.247 \\
\hline & \multicolumn{4}{|c|}{ Implicit method } \\
RMSE & 0.02122 & 0.01528 & 0.00639 & 0.00445 \\
CPU-time, s & 39.318 & 52.327 & 129.045 & 257.880 \\
\hline & \multicolumn{5}{|c|}{ Newton-like method } \\
RMSE & 0.02127 & 0.01524 & 0.00815 & 0.00471 \\
CPU-time, s & 38.650 & 52.277 & 128.371 & 255.347 \\
\hline
\end{tabular}

Table 3.3: RMSE with respect to CPU-time for different $k$ and fixed $h_{0}=0.01$.

Next example presents a study of the free boundary for both RAPM and Barles and Soner's models with various values of the respective transaction cost parameters $R$ and $a$.

Example 3.1.2. Let the problem (3.1)-(3.7) under RAPM model with the parameters (3.29), fixed transaction cost $C_{t r}=0.01$ and various risk premium measure $R=5,15,40,70,100$ to be considered. The coefficient $\mu=3\left(\frac{C_{t r}^{2} R}{2 \pi}\right)^{1 / 3}$, according to [63].

Figure 3.2 shows the variation of the normalised free boundary $S_{f}(\tau)$ depending on the parameter $R$.

In Figure 3.3 there are numerical results for Barles and Soner's model for various $a$. The difference between values for $a=0$ and $a=0.01$ is inappreciable.

In next example the validity of the proposed explicit scheme (3.20) is discussed. Explicit scheme uses information from the previous time level to compute a solution at the current moment. For nonlinear equations with coefficients depending on the solution one has two alternatives: if we take values from the current time level to compute the coefficients the scheme would not be explicit and we have to use any iterative solver for this problem. It increases computational time. Another alternative is to take values from the previous time level as we used, and the coefficients may be inaccurate. 


\section{FRONT-FIXING METHOD FOR SOME ADVANCED MODELS}

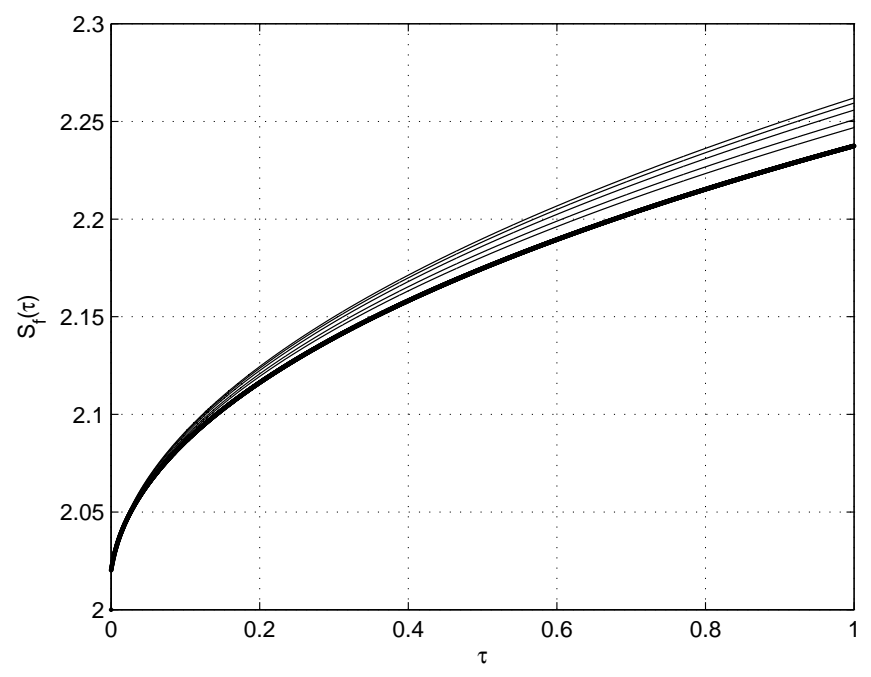

Figure 3.2: A comparison of the free boundary $S_{f}(\tau)$ for RAPM model for various risk premium measures $R=5,15,40,70,100$ with the corresponding free boundary for $R=0$ (bold line).

Example 3.1.3. The transformed American call option pricing problem under Barles and Soner's model (3.12)-(3.17) with parameters (3.29) and $a=0.05$ is considered.

Figure 3.4 demonstrates the difference between the solutions obtained by both alternatives for fixed $h_{0}$ and various $k$. One can see that the difference presents orders no bigger than $O(k)$ that is the order of approximation of the explicit forward in time scheme (3.20).

Moreover, the series of tests was provided to insure this statement. For fixed $k$ the maximum value of the difference between the solutions is calculated. The results are collected in Table 3.4

\begin{tabular}{|l|ccc|}
\hline$h_{0}$ & 0.01 & 0.02 & 0.04 \\
\hline$k=10^{-3}$ & $1.3548 \cdot 10^{-4}$ & $7.5463 \cdot 10^{-4}$ & $9.5728 \cdot 10^{-5}$ \\
$k=10^{-4}$ & $9.9036 \cdot 10^{-6}$ & $1.2605 \cdot 10^{-5}$ & $1.0068 \cdot 10^{-5}$ \\
$k=10^{-5}$ & $1.0936 \cdot 10^{-6}$ & $1.2995 \cdot 10^{-6}$ & $1.6136 \cdot 10^{-6}$ \\
\hline
\end{tabular}

Table 3.4: The maximum distance between the solution of the problem 3.12 by explicit and iterative explicit method. 


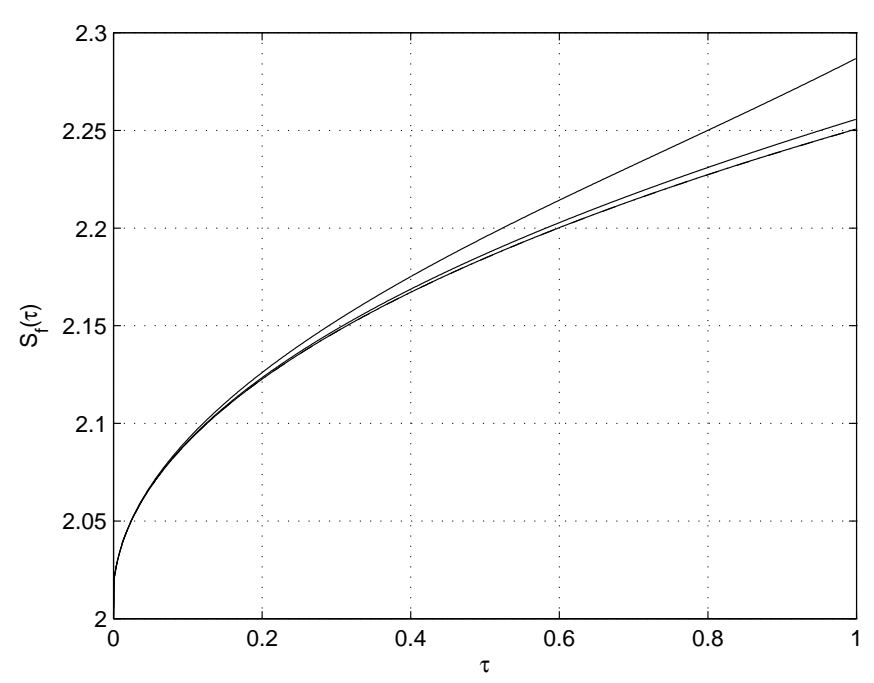

Figure 3.3: A comparison of the free boundary $S_{f}(\tau)$ for Barles and Soner's model for $a=0,0.01,0.07,0.13$.

In order to study stability of the proposed explicit method we compare solutions for the problem with the parameters 3.29 ) for fixed $h=10^{-2}$ and various $k=10^{-4}$ and $k=2.6 \cdot 10^{-3}$ (Figures 3.5 and 3.6 correspondingly). As one can see, the numerical solution as shown in Figure 3.6 is unstable.

Next example is used to examine the validity of the proposed modifications in the class of Newton-like methods.

Example 3.1.4. Well known Newton-like methods developed by Broyden and Schubert as well as proposed modifications are used to approximate the Jacobian of the problem of Example 1 with $h=0.01, k=0.001$ and $k=0.0001$.

In order to demonstrate the viability of the modifications to well known Broyden's and Schubert's methods described in Section 3.3, the spectral radius is used. Let a approximation of Jacobian by any method be denoted as $J_{\text {approx }}$. Since matrices supposed to be approximation of the original Jacobian $J$, then matrix $J_{\text {approx }}^{-1} J$ should be close to identity matrix. Spectral radius of matrix is used to check this fact.

In Table 3.5 maximum, minimum and mean value of spectral radius of matrices $J_{\text {approx }}^{-1} J$ are presented. Further tests, performed but not presented in fails for smaller step sizes and solution is unstable.

The results of this section have been published in [42]. 


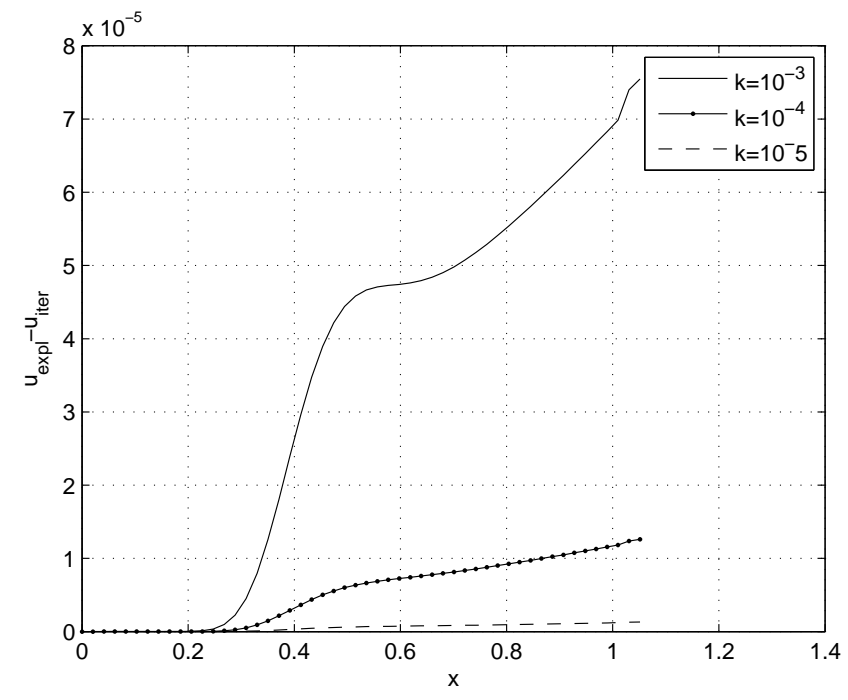

Figure 3.4: Difference between solutions by explicit method and iterative explicit method with $h_{0}=10^{-2}$ and various $k$.

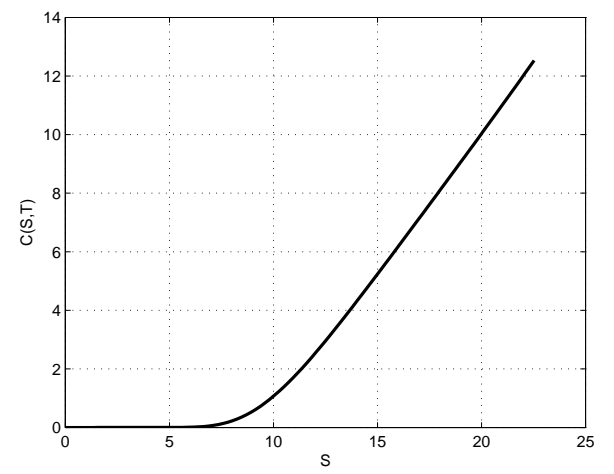

Figure 3.5: Stable numerical solution with $k=10^{-4}$.

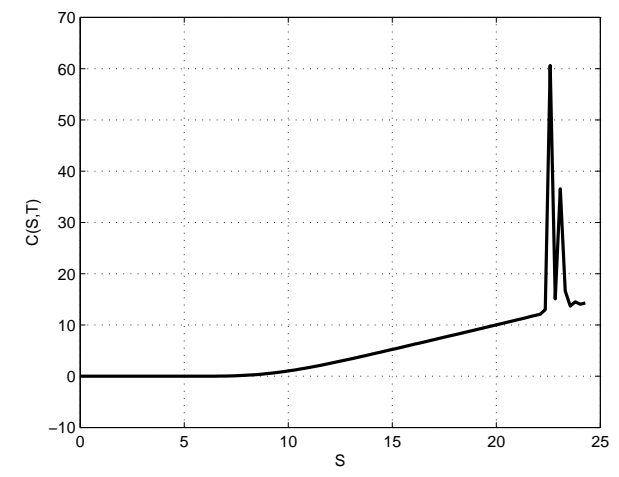

Figure 3.6: Unstable numerical solution with $k=2.6 \cdot 10^{-3}$. 


\begin{tabular}{|l|ccc|}
\hline & Min & Max & Mean \\
\hline & \multicolumn{3}{|c|}{$h=0.01, k=0.001$} \\
frozen-Broyden & 1.00239414 & 1.01232560 & 1.00648229 \\
Schubert-1 & 1.00000712 & 1.12629296 & 1.00054588 \\
Broyden & 1.00015443 & 1.00103625 & 1.00066090 \\
Schubert & \multicolumn{3}{|c|}{ fail } \\
\hline & \multicolumn{3}{|c|}{$h=0.01, k=0.0001$} \\
frozen-Broyden & 1.00044224 & 1.00372329 & 1.00095909 \\
Schubert-1 & 1.00020364 & 1.00333878 & 1.00059102 \\
Broyden & 1.00024579 & 1.00377139 & 1.00075563 \\
Schubert & \multicolumn{3}{|c}{ fail } \\
\hline
\end{tabular}

Table 3.5: Spectral radius of matrix $J_{\text {approx }}^{-1} J$, where $J_{\text {approx }}$ is calculated by various methods. 


\subsection{Regime switching model}

In this section a continuous time Markov chain $\alpha_{t}$ is considered taking values among $I$ different regimes, where $I$ is the total number of regimes considered in the market. Each regime is labelled by an integer $i$ with $1 \leq i \leq I$. Hence, the regime space of $\alpha_{t}$ is $M=\{1,2, \ldots, I\}$. Let $Q=\left(q_{i, j}\right)_{I \times I}$ be the given generator matrix of $\alpha_{t}$. From [113] the entries $q_{i, j}$ satisfy:

$$
q_{i, j} \leq 0, \text { if } i \neq j ; \quad q_{i, i}=-\sum_{j \neq i} q_{i, j}, 1 \leq i \leq I .
$$

Under the risk-neutral measure, see Elliott et al. [45] for details, the stochastic process for the underlying asset $S_{t}$ is

$$
\frac{d S_{t}}{S_{t}}=r_{\alpha_{t}} d t+\sigma_{\alpha_{t}} d \tilde{B}_{t}, t \leq 0
$$

where $\sigma_{\alpha_{t}}$ is the volatility of the asset $S_{t}$ and $r_{\alpha_{t}}$ is the risk-free interest rate.

Here the American put option on the asset $S_{t}=S$ with strike price $E$ and maturity $T<\infty$ is considered. Let $V_{i}(S, \tau)$ denote the option price functions, where $\tau=T-t$ denotes the time to maturity, the asset price $S$ and the regime $\alpha_{t}=i$. Then, $V_{i}(S . \tau), 1 \leq i \leq I$, satisfy the following free boundary problem:

$$
\frac{\partial V_{i}}{\partial \tau}=\frac{\sigma_{i}^{2}}{2} S^{2} \frac{\partial^{2} V_{i}}{\partial S^{2}}+r_{i} S \frac{\partial V_{i}}{\partial S}-r_{i} V_{i}+\sum_{l \neq i} q_{i l}\left(V_{l}-V_{i}\right), \quad S>S_{i}^{*}(\tau), 0<\tau \leq T
$$

where $S_{i}^{*}(\tau)$ denote optimal stopping boundaries of the option. Initial conditions are

$$
V_{i}(S, 0)=\max (E-S, 0), \quad S_{i}^{*}(0)=E, \quad i=1, \ldots, I .
$$

Boundary conditions for $i=1, \ldots, I$ are as follows

$$
\begin{aligned}
\lim _{S \rightarrow \infty} V_{i}(S, \tau) & =0 \\
V_{i}\left(S_{i}^{*}(\tau), \tau\right) & =E-S_{i}^{*}(\tau), \\
\frac{\partial V_{i}}{\partial S}\left(S_{i}^{*}(\tau), \tau\right) & =-1
\end{aligned}
$$

Several different numerical methods for solving the system of partial differential equations (3.31) have been proposed. Lattice methods [87, 114] are popular for practitioners because they are easy to implement, but they have the drawback of the 
absence of numerical analysis and subsequent unreliability, because the lack of numerical analysis may waste the best model. The penalty method [57, 70, 71, 116] uses a coupling of the penalty term and the regime coupling terms. Both, the lattice and penalty methods do not calculate the optimal stopping boundary that has interest from the practitioners point of view.

The challenging task of the free boundary as another unknown into the PDE problem is not new in the literature. In fact, since Landau's ideas [79] the so-called front-fixing method has been used in many fields [33] and by [2, 24, 26, 77, 108] for American option problems without switching.

In this section we address the numerical solution of the coupled PDE system (3.31). Firstly, by extending the ideas developed in [26], the PDE system [3.31] is transformed into a new PDE system on a fixed domain where the free boundaries $S_{i}^{*}(\tau), 1 \leq i \leq I$, are incorporated as new unknowns of the system. This allows the computation not only of the prices, but also of all the optimal exercise prices.

In spite of the apparent complexity of the transformed problem due to the appearance of new spatial variables, one for each equation, the explicit numerical scheme constructed becomes easy to implement, computationally cheap and accurate when one compares with the more relevant existing methods. Implicit weighted schemes have been developed in this section for the sake of performance comparison.

\subsubsection{Multi-variable fixed domain transformation}

Fixed domain transformation techniques inspired in Landau ideas [79] have been used by several authors ([112], [108], [90], [26]) for partial differential equations modelling American option pricing problems. To our knowledge this transformation technique has not been applied before for a partial differential system with several unknown free boundaries, one for each equation.

Based on the transformation used by the authors in [112], [26] for the case of just one equation, let us consider the multi-variable transformation

$$
x^{i}=\ln \frac{S}{S_{i}^{*}(\tau)}, \quad 1 \leq i \leq I .
$$

Note that the new variables $x^{i}$ lie in the fixed positive real line. The price $V_{i}$ of $i$-th regime involved in $i$-th equation of the system and $i$-th free boundary are related by the dimensionless transformation 


$$
P_{i}\left(x^{i}, \tau\right)=\frac{V_{i}(S, \tau)}{E}, \quad X_{i}(\tau)=\frac{S_{i}^{*}(\tau)}{E}, \quad 1 \leq i \leq I .
$$

Then the value of option $l$-th regime appearing in $i$-th coupled equation, $l \neq i$, becomes

$$
P_{l, i}\left(x^{i}, \tau\right)=\frac{V_{l}(S, \tau)}{E} .
$$

Since from 3.37), $\frac{V_{l}(S, \tau)}{E}=P_{l}\left(x^{l}, \tau\right)$ and taking into account transformation 3.36) for indexes $i$ and $l$ one gets that

$$
P_{l, i}\left(x^{i}, \tau\right)=P_{l}\left(x^{l}, \tau\right),
$$

and it occurs when the variables are related by the equation

$$
x^{l}=x^{i}+\ln \frac{X_{i}(\tau)}{X_{l}(\tau)}, \quad 1 \leq i, l \leq I .
$$

From (3.36) - (3.38) the problem (3.31) - 3.35) for $1 \leq i \leq I$ takes a new form

$$
\begin{aligned}
\frac{\partial P_{i}}{\partial \tau}\left(x^{i}, \tau\right) & =\frac{\sigma_{i}^{2}}{2} \frac{\partial^{2} P_{i}}{\partial\left(x^{i}\right)^{2}}\left(x^{i}, \tau\right)+\left(r_{i}-\frac{\sigma_{i}^{2}}{2}+\frac{X_{i}^{\prime}(\tau)}{X_{i}(\tau)}\right) \frac{\partial P_{i}}{\partial x^{i}}\left(x^{i}, \tau\right)-r_{i} P_{i}\left(x^{i}, \tau\right) \\
& +\sum_{l \neq i} q_{i l}\left(P_{l, i}\left(x^{i}, \tau\right)-P_{i}\left(x^{i}, \tau\right)\right)=0, x^{i}>0,0<\tau \leq T
\end{aligned}
$$

with initial and boundary conditions

$$
\begin{aligned}
P_{i}\left(x^{i}, 0\right) & =\max \left(1-e^{x^{i}}, 0\right)=0, \\
X_{i}(0) & =1, \\
P_{i}(0, \tau) & =1-X_{i}(\tau), \\
\frac{\partial P_{i}}{\partial x^{i}}(0, \tau) & =-X_{i}(\tau), \\
\lim _{x^{i} \rightarrow \infty} P_{i}\left(x^{i}, \tau\right) & =0 .
\end{aligned}
$$

Note that from equation 3.39$) x^{l}$ could be negative if $X_{l}(\tau)>X_{i}(\tau)$ and this means that due to the equation (3.36) $S<S_{l}^{*}(\tau)$, and in this case the value of the option at $l$-th regime agrees with the payoff, i.e.

$$
P_{l, i}\left(x^{i}, \tau\right)=P_{l}\left(x^{l}, \tau\right)=1-X_{l}(\tau) e^{x^{l}}, \quad x^{l} \leq 0 .
$$




\subsubsection{Discretization and numerical schemes construction}

Dealing with numerical solutions of the transformed problem 3.40 - (3.45) a bounded numerical domain must be defined.

A numerical solution has to be found on infinite domain $[0 ; \infty) \times[0 ; T]$ for all regimes. In accordance with [66], [106] the domain in original variable $S$ can be truncated about three or four times the exercise price. It is sufficient to take the numerical domain for the transformed problem (3.40)-(3.45) as $\left[0 ; x_{\max }\right], x_{\max }=3$. The computational domain is covered by an uniform grid with common step sizes $h=\frac{x_{\max }}{M}$ and $k=\frac{T}{N}$. Nodes of the grid are denoted as follows

$$
x_{j}=j h, 0 \leq j \leq M ; \quad \tau^{n}=n k, 0 \leq n \leq N .
$$

Let us denote $u_{i, j}^{n} \approx P_{i}\left(x_{j}, \tau^{n}\right)$ the approximation of $P_{i}$ in $i$-th equation at mesh point $\left(x^{i}=x_{j}, \tau=\tau^{n}\right)$ and $\tilde{u}_{l_{i}, j}^{n} \approx P_{l, i}\left(x_{j}, \tau^{n}\right)$ be the approximation of $P_{l}$ in $i$-th equation evaluated at the point $\left(x^{i}=x_{j}, \tau=\tau^{n}\right)$. The discretization of the transformed optimal stopping boundary is denoted by $X_{i}^{n} \approx X_{i}\left(\tau^{n}\right)$. Then an explicit finite difference scheme can be written in the form

$$
\begin{aligned}
\frac{u_{i, j}^{n+1}-u_{i, j}^{n}}{k} & =\frac{\sigma_{i}^{2}}{2} \frac{u_{i, j+1}^{n}-2 u_{i, j}^{n}+u_{i, j-1}^{n}}{h^{2}} \\
& +\left(r_{i}-\frac{\sigma_{i}^{2}}{2}+\frac{X_{i}^{n+1}-X_{i}^{n}}{k X_{i}^{n}}\right) \frac{u_{i, j+1}^{n}-u_{i, j-1}^{n}}{2 h} \\
& -r_{i} u_{i, j}^{n}+\sum_{l \neq i} q_{i l}\left(\tilde{u}_{l_{i}, j}^{n}-u_{i, j}^{n}\right),
\end{aligned}
$$

where

$$
\tilde{u}_{l_{i}, j}^{n} \approx P_{l, i}\left(x_{j}, \tau^{n}\right)=P_{l}\left(x_{j}+\ln \frac{X_{i}^{n}}{X_{l}^{n}}, \tau^{n}\right)
$$

are obtained by linear interpolation of values $u_{l, j}^{n}$ at the point $x_{j}+\ln \frac{X_{i}^{n}}{X_{l}^{n}}$ known from the previous time level $n$,

$$
\tilde{u}_{l_{i}, j}^{n}= \begin{cases}1-X_{i}^{n} e^{x_{j}}, & x_{j}<-\ln \frac{X_{i}^{n}}{X_{l}^{n}} \\ \alpha_{l, j}^{n} u_{l, j_{0}}^{n}+\beta_{l, j}^{n} u_{l, j_{0}+1}^{n}, & -\ln \frac{X_{i}^{n}}{X_{l}^{n}} \leq x_{j} \leq x_{\max }-\ln \frac{X_{i}^{n}}{X_{l}^{n}} \\ 0, & x_{j}>x_{\max }-\ln \frac{X_{i}^{n}}{X_{l}^{n}}\end{cases}
$$

Note that in the first situation of $3.47, x_{j}<\ln \frac{X_{i}^{n}}{X_{l}^{n}}$, means that in the original variables $S<S_{l}^{*}\left(\tau^{n}\right)$ where the option price is payoff value. In the second case we use the linear interpolation where the positive coefficients are given by 


$$
\alpha_{l, j}^{n}=\frac{h\left(j_{0}+1\right)-h j-\ln \frac{X_{i}^{n}}{X_{l}^{n}}}{h}, \quad \beta_{l, j}^{n}=\frac{h j+\ln \frac{X_{i}^{n}}{X_{l}^{n}}-h j_{0}}{h} .
$$

where $j_{0}=j_{0}(i, l, j)$, is the biggest integer number such that

$$
h j_{0} \leq h j+\ln \frac{X_{i}^{n}}{X_{l}^{n}}<h\left(j_{0}+1\right),
$$

Finally, in the last case we assign to $\tilde{u}_{l_{i}, j}^{n}=0$ due to condition 3.45 .

From the properties of the model for any regime $i$ one gets

$$
\sum_{l \neq i} q_{i l}=-q_{i i}, \quad q_{i i}<0
$$

and denoting constants

$$
\begin{aligned}
a_{i} & =\frac{\sigma_{i}^{2}}{2} \frac{k}{h^{2}}-\left(r_{i}-\frac{\sigma_{i}^{2}}{2}\right) \frac{k}{2 h}, \\
b_{i} & =1-\sigma_{i}^{2} \frac{k}{h^{2}}-\left(r_{i}-q_{i i}\right) k, \\
c_{i} & =\frac{\sigma_{i}^{2}}{2} \frac{k}{h^{2}}+\left(r_{i}-\frac{\sigma_{i}^{2}}{2}\right) \frac{k}{2 h},
\end{aligned}
$$

the scheme 3.46 can be presented for $j=1, \ldots, M-1, i=1, \ldots, I, n=$ $0, \ldots, N-1$ as follows

$$
u_{i, j}^{n+1}=a_{i} u_{i, j-1}^{n}+b_{i} u_{i, j}^{n}+c_{i} u_{i, j+1}^{n}+\frac{X_{i}^{n+1}-X_{i}^{n}}{2 h X_{i}^{n}}\left(u_{i, j+1}^{n}-u_{i, j-1}^{n}\right)+k \sum_{l \neq i} q_{i l} \tilde{u}_{l_{i}, j}^{n} .
$$

From the boundary conditions 3.43, 3.45 one gets

$$
u_{i, 0}^{n+1}=1-X_{i}^{n+1}, \quad u_{M}^{n+1}=0 .
$$

Boundary condition (3.44) can be discretized by using the second order oneside-difference approximation :

$$
\frac{-3 u_{i, 0}^{n+1}+4 u_{i, 1}^{n+1}-u_{i, 2}^{n+1}}{2 h}+X_{i}^{n+1}=0 .
$$

Since number of unknowns $M+2$ is equal to the number of the equations of the system of (3.53), 3.54) and 3.55), it is closed and can be solved. 
Thus, the unknown optimal stopping boundary can be derived from 3.53, (3.54) and (3.55):

$$
X_{i}^{n+1}=\frac{\xi_{i}^{n}}{\eta_{i}^{n}}
$$

where

$$
\begin{aligned}
\xi_{i}^{n} & =3-4 a_{i} u_{i, 0}^{n}-\left(4 b_{i}-a_{i}\right) u_{i, 1}^{n}-\left(4 c_{i}-b_{i}\right) u_{i, 2}^{n}+c_{i} u_{i, 3}^{n} \\
& +\frac{4\left(u_{i, 2}^{n}-u_{i, 0}^{n}\right)-\left(u_{i, 3}^{n}-u_{i, 1}^{n}\right)}{2 h}-k\left(4 \Sigma_{1}-\Sigma_{2}\right), \\
\eta_{i}^{n} & =3+2 h+\frac{4\left(u_{i, 2}^{n}-u_{i, 0}^{n}\right)-\left(u_{i, 3}^{n}-u_{i, 1}^{n}\right)}{2 h X_{i}^{n}}
\end{aligned}
$$

and $\Sigma_{j}=\sum_{l \neq i} q_{i l} \tilde{u}_{l_{i}, j}^{n}$.

In order to compare the performance of the proposed explicit difference scheme (3.46) and for the sake of comparison we also introduce a modification of the well known $\theta$-family of implicit finite difference schemes, so-called weighted average approximation [101], but making explicit in the coupled regimes term to save computational cost. Thus, for each fixed regime $i=1, \ldots, I$ equation 3.40 is discretised with previous notation as follows:

$$
\begin{array}{r}
\frac{u_{i, j}^{n+1}-u_{i, j}^{n}}{k}=\frac{\sigma_{i}^{2}}{2}\left[\theta \frac{u_{i, j+1}^{n+1}-2 u_{i, j}^{n+1}+u_{i, j-1}^{n+1}}{h^{2}}+(1-\theta) \frac{u_{i, j+1}^{n}-2 u_{i, j}^{n}+u_{i, j-1}^{n}}{h^{2}}\right] \\
+\left(r_{i}-\frac{\sigma_{i}^{2}}{2}+\frac{X_{i}^{n+1}-X_{i}^{n}}{k X_{i}^{n}}\right)\left[\theta \frac{u_{i, j+1}^{n+1}-u_{i, j-1}^{n+1}}{2 h}+(1-\theta) \frac{u_{i, j+1}^{n}-u_{i, j-1}^{n}}{2 h}\right] \\
-\left(r_{i}-q_{i, i}\right)\left[\theta u_{i, j}^{n+1}+(1-\theta) u_{i, j}^{n}\right]+\sum_{l \neq i} q_{i l} \tilde{u}_{l i, j}^{n}, \quad j=1, \ldots, M-1, n=0, \ldots, N-1,
\end{array}
$$

where $\theta \in[0,1]$ is the weight parameter.

The boundary conditions are taken in the form 3.54-(3.55). As implicit method is employed for the numerical solution, the optimal stopping boundary is fully involved in the system, but has not an isolated expression like (3.56)-(3.58). The closed system of $M+2$ equations (3.54)-(3.55) and (3.59) is solved by using the well know iterative Newton's method for every regime $i=1, \ldots, I$.

Since the system is solved for a fixed regime, let us skip out the index of regime $i$ and introduce the unknown vector $U^{n}$ 


$$
U^{n}=\left(X^{n}, \quad u_{0}^{n}, \quad u_{1}^{n}, \quad \ldots, \quad u_{M-1}^{n}\right)^{T} .
$$

For the sake of simplicity the value $u_{M}^{n}=0, n=0, \ldots, N$ is excluded of the system. Thus, the system takes the following vector form

$$
A^{n+1} U^{n+1}=B^{n}
$$

where the matrix of coefficients $A^{n+1}$ and vector $B^{n}$ are given by

$$
\begin{gathered}
A^{n+1}=\left(\begin{array}{ccccccccc}
1 & 1 & 0 & 0 & 0 & 0 & \ldots & 0 & 0 \\
-2 h & 3 & -4 & 1 & 0 & 0 & \ldots & 0 & 0 \\
0 & a_{1}^{n+1} & a_{2}^{n+1} & a_{3}^{n+1} & 0 & 0 & \ldots & 0 & 0 \\
0 & 0 & a_{1}^{n+1} & a_{2}^{n+1} & a_{3}^{n+1} & 0 & \ldots & 0 & 0 \\
\ddots & \ddots & \ddots & \ddots & \ddots & \ddots & \ddots & \ddots & \ddots \\
0 & 0 & 0 & 0 & 0 & 0 & \ldots & a_{1}^{n+1} & a_{2}^{n+1}
\end{array}\right) \\
B^{n}=\left(\begin{array}{c}
1 \\
b_{1}^{n+1} u_{0}^{n}+b_{2}^{n+1} u_{1}^{n}+b_{3} u_{2}^{n}+k \sum_{l \neq i} q_{i l} \tilde{u}_{l_{i}, 1}^{n} \\
b_{1}^{n+1} u_{M-2}^{n}+b_{2}^{n+1} u_{M-1}^{n}+k \sum_{l \neq i} q_{i l} \tilde{u}_{l_{i}, M-1}^{n}
\end{array}\right)
\end{gathered}
$$

Coefficients $a_{j}^{n+1}$ and $b_{j}^{n+1}, j=1,2,3$ are derived from the scheme 3.59 as follows

$$
\begin{aligned}
a_{1}^{n+1} & =-\frac{\sigma^{2}}{2} \theta \frac{k}{h^{2}}+\left(r-\frac{\sigma^{2}}{2}+\frac{X^{n+1}-X^{n}}{k X^{n}}\right) \theta \frac{k}{2 h}, \\
a_{2}^{n+1} & =1+\left(r-q_{i, i}\right) \theta k+\sigma^{2} \theta \frac{k}{h^{2}}, \\
a_{3}^{n+1} & =-\frac{\sigma^{2}}{2} \theta \frac{k}{h^{2}}-\left(r-\frac{\sigma^{2}}{2}+\frac{X^{n+1}-X^{n}}{k X^{n}}\right) \theta \frac{k}{2 h}, \\
b_{1}^{n+1} & =\frac{\sigma^{2}}{2}(1-\theta) \frac{k}{h^{2}}-\left(r-\frac{\sigma^{2}}{2}+\frac{X^{n+1}-X^{n}}{k X^{n}}\right)(1-\theta) \frac{k}{2 h}, \\
b_{2}^{n+1} & =1-\left(r-q_{i, i}\right)(1-\theta) k+\sigma^{2}(1-\theta) \frac{k}{h^{2}}, \\
b_{3}^{n+1} & =\frac{\sigma^{2}}{2}(1-\theta) \frac{k}{h^{2}}+\left(r-\frac{\sigma^{2}}{2}+\frac{X^{n+1}-X^{n}}{k X^{n}}\right)(1-\theta) \frac{k}{2 h} .
\end{aligned}
$$


Let us write the $j$-th step of the Newton iteration process as

$$
G_{j}=A_{j}^{n+1} U_{j}^{n+1}-B_{j}^{n}=0 .
$$

The solution $U^{n}$ is taken as initial guess $U_{0}^{n+1}$ and the next iteration $U_{j+1}^{n+1}$ for known $U_{j}^{n+1}$ is calculated by

$$
U_{j+1}^{n+1}=U_{j}^{n+1}-\left(J\left(G_{j}\right)\right)^{-1} G_{j}
$$

Because of the dependence of the entries of matrix $A_{j}^{n+1}$ on the stopping boundary $X_{j}^{n+1}$, Jacobian of the system $3.60 J\left(G_{j}\right)$ can be expressed by

$$
J\left(G_{j}\right)=A_{j}^{n+1}+Y J_{X}^{n+1} .
$$

Here $Y$ is the sparse matrix

$$
\begin{gathered}
Y=\left(\begin{array}{cccc}
0 & 0 & \ldots & 0 \\
0 & 0 & \ldots & 0 \\
1 & 0 & \ldots & 0 \\
\vdots & \vdots & \ldots & \vdots \\
1 & 0 & \ldots & 0
\end{array}\right), \\
J_{X}^{n+1}=\frac{1}{2 h X^{n}}\left(\left(\begin{array}{cccccc}
0 & -1 & 0 & \ldots & 0 & 0 \\
1 & 0 & -1 & \ldots & 0 & 0 \\
\ddots & \ddots & \ddots & \ddots & 0 & -1 \\
0 & 0 & 0 & \ldots & 1 & 0
\end{array}\right)\left[\theta \tilde{U}_{j}^{n+1}+(1-\theta) \tilde{U}^{n}\right]\right) \\
+\frac{1}{2 h X^{n}}\left[\theta \tilde{u}_{0}^{n+1}+(1-\theta) u_{0}^{n}\right]\left(\begin{array}{c}
1 \\
0 \\
\vdots \\
0
\end{array}\right)
\end{gathered}
$$

where the vector of the solution at interior points, i.e. with spatial indexes $1, \ldots, M-1$ is denoted by $\tilde{U}_{j}^{n+1}, \tilde{U}^{n}=\left[u_{1}^{n}, \ldots, u_{M-1}^{n}\right]$ and the $j$-th iteration of the solution at the point $\left(0, \tau^{n+1}\right)$ by $\tilde{u}_{0}^{n+1}$.

As usual, the stopping criteria is that norm of vector $\Delta U^{n+1}=U_{j+1}^{n+1}-U_{j}^{n+1}$ is smaller than chosen tolerance $\epsilon$. 


\section{FRONT-FIXING METHOD FOR SOME ADVANCED MODELS}

\subsubsection{Von Neumann stability analysis}

In this section we study the stability of the proposed explicit scheme following von Neumann analysis approach originally applied to schemes with constant coefficients. However, such approach can be used also for the variable coefficients case by freezing at each level (see [102], p. 59, [39], [53]).

In order to avoid notational misunderstanding among the imaginary unit with the regime index $i$ used in previous section, here we denote the regime index by $R$.

An initial error vector for every regime $g_{R}^{0}, R=1, \ldots, I$, is expressed as a finite complex Fourier series, so that at $x_{j}$ the solution $u_{i, j}^{n}$ can be rewritten as follows

$$
u_{R, j}^{n}=g_{R}^{n} e^{i j \theta}, \quad j=1, \ldots, M-1, R=1, \ldots, I,
$$

where $i=(-1)^{1 / 2}$ is the imaginary unit and $\theta$ is phase angle. Then the scheme is stable if for every regime $R=1, \ldots, I$ the amplification factor $G_{R}=\frac{g_{R}^{n+1}}{g_{R}^{n}}$ satisfies the relation

$$
\left|G_{R}\right| \leq 1+K k=1+O(k),
$$

where the positive number $K$ is independent of $h, k$ and $\theta$, see [101], p. 68, [102], p. 50 .

For the sake of simplicity of the notation the index of the regime $R$ is skipped in the unknowns, the coefficients and the parameters, supposing that the calculations are done for every regime. Using boundary conditions 3.55 and 3.61 , one gets

$$
X^{n}=\frac{g^{n+1}\left(3-4 e^{i \theta}+e^{2 i \theta}\right)}{2 h},
$$

and consequently

$$
\frac{X^{n+1}-X^{n}}{X^{n}}=G-1 .
$$

Then the numerical scheme (3.53) takes the following form

$$
\begin{aligned}
g^{n+1} e^{i j \theta} & =a g^{n} e^{i(j-1) \theta}+b g^{n} e^{i j \theta}+c g^{n} e^{i(j+1) \theta} \\
& +\left(\frac{g^{n+1}}{g^{n}}-1\right) \frac{g^{n}}{2 h}\left(e^{i(j+1) \theta}-e^{i(j-1) \theta}\right) \\
& +k \sum_{l \neq R} q_{R, l} g_{l}^{n}\left(\alpha_{l, j}^{n} e^{i j_{0} \theta}+\beta_{l, j}^{n} e^{i\left(j_{0}+1\right) \theta}\right) .
\end{aligned}
$$

Let us denote

$$
z=\sum_{l \neq R} q_{R, l} \frac{g_{l}^{n}}{g^{n}}\left(\alpha_{l, j}^{n} e^{i\left(j_{0}-j\right) \theta}+\beta_{l, j}^{n} e^{i\left(j_{0}+1-j\right) \theta}\right),
$$


then dividing both parts of 3.64 by $g^{n} e^{i j \theta}$, and taking into account 3.63, one gets

$$
G=a e^{-i \theta}+b+c e^{i \theta}+\frac{i \sin \theta}{h}(G-1)+k z .
$$

According to properties of the linear interpolation, $\alpha_{l, j}^{n}+\beta_{l, j}^{n}=1$ (see $(3.48)$ ), and 3.65 can be bounded by

$$
|z| \leq \sum_{l \neq R} q_{R, l}\left|\frac{g_{l}^{n}}{g^{n}}\right| \leq \max _{l \neq R}\left|\frac{g_{l}^{n}}{g^{n}}\right|\left|q_{R, R}\right|=\left|\frac{g_{l_{0}(n)}^{n}}{g^{n}}\right|\left|q_{R, R}\right|=C(n),
$$

where $C(n)$ is independent of $\theta, h$ and $k$ and depends only on the frozen index $n$. From 3.66, 3.67) and 3.50)-(3.52) it follows that

$$
|G|\left|1-\frac{i \sin \theta}{h}\right| \leq|A(k, h, \theta)|+C(n) k,
$$

where

$$
\begin{aligned}
|A(k, h, \theta)|^{2} & =\left(1-2 \frac{\sigma^{2} k \sin ^{2} \frac{\theta}{2}}{h^{2}}-(r-q) k\right)^{2} \\
& +\frac{\sin ^{2} \theta}{h^{2}}\left(\left(r-\frac{\sigma^{2}}{2}\right)^{2} k^{2}-2 k\left(r-\frac{\sigma^{2}}{2}\right)+1\right) .
\end{aligned}
$$

Thus, in agreement with (3.62) the scheme is stable, if

$$
|A(k, h, \theta)|^{2} \leq 1+\frac{\sin ^{2} \theta}{h^{2}} .
$$

It is easy to check that 3.68 holds true, if

$$
\left\{\begin{array}{l}
\sigma^{2} k\left((r-q)-\frac{\sigma^{2}}{h^{2}}\right)-\sigma^{2} \leq 0, \\
\left(\left(r-\frac{\sigma^{2}}{2}\right)^{2}+(r-q) \sigma^{2}\right) k-2 r \leq 0 .
\end{array}\right.
$$

Conditions 3.69 hold true when

$$
k \leq \min \left(\frac{h^{2}}{\sigma^{2}+(r-q) h^{2}}, \frac{2 r}{\left(r-\frac{\sigma^{2}}{2}\right)^{2}+(r-q) \sigma^{2}}\right) .
$$

Summarizing the following result can be established: 


\section{FRONT-FIXING METHOD FOR SOME ADVANCED MODELS}

Theorem 3.2.1. With previous notation the scheme (3.53) is conditionally stable under the constraint

$$
k \leq \min _{1 \leq R \leq I}\left(\frac{h^{2}}{\sigma_{R}^{2}+\left(r_{R}-q_{R, R}\right) h^{2}}, \frac{2 r_{R}}{\left(r_{R}-\frac{\sigma_{R}^{2}}{2}\right)^{2}+\left(r_{R}-q_{R, R}\right) \sigma_{R}^{2}}\right) .
$$

\subsubsection{Local truncation error and consistency}

Theorem 3.2.2. Assuming that the solution of the PDE problem (3.40)-(3.45) admits two times continuous partial derivative with respect to time and up to order four with respect to space, the numerical solution computed by the scheme (3.46) with (3.55) is consistent with the equation (3.40) and boundary condition (3.44) of the second order in space and the first order in time.

Proof. Under hypothesis of the theorem using Taylor's expansion about $\left(x_{j}, \tau^{n}\right)$ the local truncation error takes form

$$
\begin{aligned}
F_{i, j}^{n}\left(X^{*}, P\right)-L_{i}\left(X^{*}, P\right) & =k E_{i, j}^{n}(3)-\frac{\sigma^{2}}{2} h^{2} E_{i, j}^{n}(2)+\left(r_{i}-\frac{\sigma_{i}^{2}}{2}\right) h^{2} E_{i, j}^{n}(1) \\
& -k E_{j}^{n}(4) \frac{\partial P_{i}}{\partial x}\left(x_{j}, \tau^{n}\right)-\frac{h^{2}}{\hat{X}_{i}^{n}} E_{i, j}^{n}(1) \frac{d X_{i}}{d \tau}\left(\tau^{n}\right) \\
& -k h^{2} E_{i, j}^{n}(4) E_{j}^{n}(1)-\sum_{l \neq i} q_{i l} E_{l, j}^{n}(5),
\end{aligned}
$$

where

$$
\begin{array}{lll}
E_{i, j}^{n}(1)=\frac{1}{6} \frac{\partial^{3} P_{i}}{\partial x^{3}}\left(\xi_{1}, \tau^{n}\right), & x_{j-1}<\xi_{1}<x_{j+1}, \\
E_{i, j}^{n}(2)=\frac{1}{12} \frac{\partial^{4} P_{i}}{\partial x^{4}}\left(\xi_{2}, \tau^{n}\right), & x_{j-1}<\xi_{2}<x_{j+1}, \\
E_{i, j}^{n}(3)=\frac{1}{2} \frac{\partial^{2} P_{i}}{\partial \tau^{2}}\left(x_{j}, \eta_{3}\right), & \tau^{n}<\eta_{3}<\tau^{n+1}, \\
E_{i, j}^{n}(4)=\frac{1}{2 \hat{X}_{i}} \frac{d^{2} X_{i}}{d \tau^{2}}\left(\eta_{4}\right), & \tau^{n}<\eta_{4}<\tau^{n+1}, \\
E_{l, j}^{n}(5)=\tilde{u}_{l, j}^{n}-P_{l, i}\left(x_{j}, \tau^{n}\right), & l \neq i .
\end{array}
$$

Taking into account that the error of linear interpolation is $O\left(h^{2}\right)$ (see [32], p. 53 ) and $3.71-(3.76)$, the local truncation error is $O(k)+O\left(h^{2}\right)$. 
Since for discretization of boundary condition (3.44) the one-side difference of the second order 3.55 is used, it is easy to check using Taylor's expansion that the local truncation error of boundary conditions is the second order in space. This fact completes the proof.

\subsubsection{Numerical examples}

In this section numerical results are presented to show the properties of the proposed method as well as comparison with other known approaches. In example 1 the stability condition (3.70) cannot be removed and numerical solution is compared with results of well recognized penalty and lattice methods presented in [70].

Example 3.2.1. Let us consider an American Put option in 2-regime switching model with the parameters (see Example 1 in [70]):

$\boldsymbol{r}=\left(\begin{array}{l}r_{1} \\ r_{2}\end{array}\right)=\left(\begin{array}{c}0.1 \\ 0.05\end{array}\right), \boldsymbol{\sigma}=\left(\begin{array}{l}\sigma_{1} \\ \sigma_{2}\end{array}\right)=\left(\begin{array}{c}0.8 \\ 0.3\end{array}\right), Q=\left(\begin{array}{rr}-6 & 6 \\ 9 & -9\end{array}\right), T=1, E=9$

Taking $h=10^{-2}$ and $k=10^{-4}$ stability constraints (3.70) are fulfilled and the option prices for both regimes and payoff function are presented on the Fig. 3.7 while the optimal stopping boundary is shown in Fig. 3.8. However, when $h=10^{-2}, k=1.6 \cdot 10^{-4}$ stability condition is broken and Fig. 3.9 reveals undesired unstable solution.

In order to compare the solution with penalty and lattice methods described in [70], Table 3.6 contains option prices for different values of asset price $S$ computed by: our proposed front-fixing explicit method (FF-expl), the exponential time differencing Crank-Nicolson scheme (ETD-CN) and the binomial tree approach developed by Liu in [87] (Tree). This binomial tree model has the good property that tree only grows linearly as the number of time steps increases allowing the use of large number of time steps to compute accurately prices of options. This binomial tree model has been used as an option pricing reference value by other relevant authors, and in particular by Khaliq et al. for the regime switching model in [70]. Table 3.6 shows that our results are close to both methods especially to the binomial model of [87].

Efficiency of explicit scheme in comparison with implicit theta methods is demonstrated in Table 3.7. The option price at the point $S=E$ for the data 3.77) 


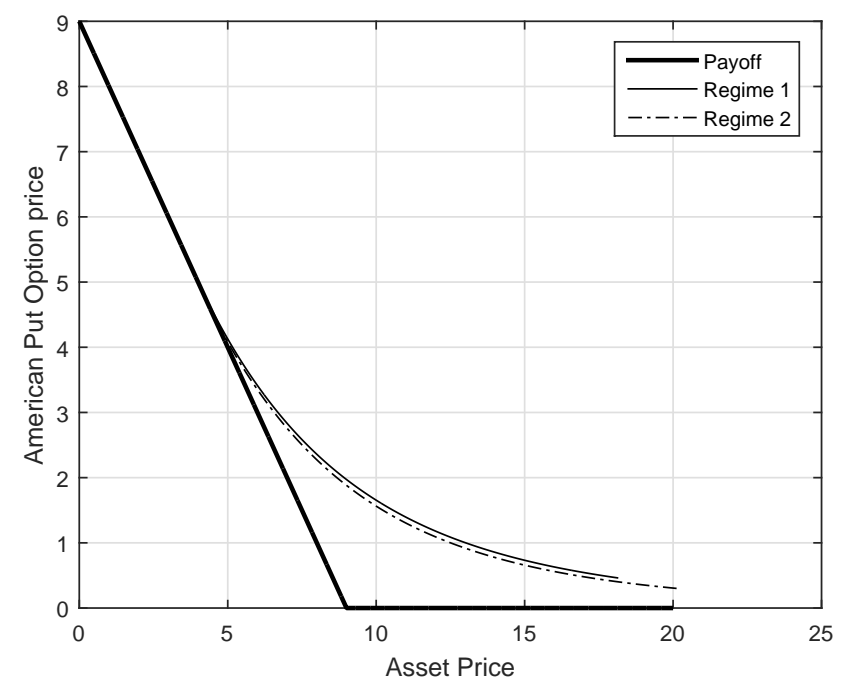

Figure 3.7: American put option price curves at $\tau=T$ and its payoff.

\begin{tabular}{|c|ccc|ccc|}
\hline & \multicolumn{3}{|c|}{ Regime 1 } & \multicolumn{3}{c|}{ Regime 2 } \\
S & FF-expl & ETD-CN & Tree & FF-expl & ETD-CN & Tree \\
\hline 9.0 & 1.9713 & 1.9756 & 1.9722 & 1.8817 & 1.8859 & 1.8819 \\
9.5 & 1.8049 & 1.8089 & 1.8058 & 1.7141 & 1.7181 & 1.7143 \\
10.5 & 1.5177 & 1.5213 & 1.5186 & 1.4265 & 1.4301 & 1.4267 \\
12.0 & 1.1796 & 1.1825 & 1.1803 & 1.0915 & 1.0945 & 1.0916 \\
\hline
\end{tabular}

Table 3.6: Comparison of American put option prices in a two regime model.

and CPU time of the methods are presented. The Newton's algorithm runs $I$ times at every time step. Therefore computational cost of implicit method is higher even if the time step $k$ is greater. Note that the results of Crank-Nicolson method are close to the results of penalty ETD-CN method from the Table 3.6 .

Next example deals with numerical convergence rate of the scheme and the computational cost. Efficiency comparison with well reputed methods such as a fitted finite volume method based on penalty approach developed in [116] and an iterated optimal stopping as well as a local policy iteration methods in [5].

Example 3.2.2. Convergence rate is studied numerically in terms of root mean 


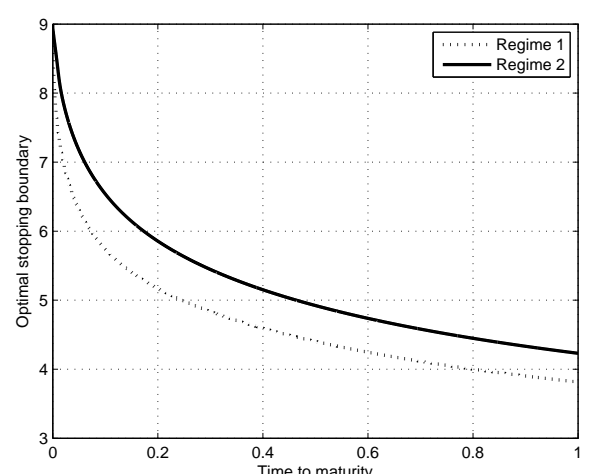

Figure 3.8: Optimal stopping boundary for regime 1 and regime 2 (stability condition is fulfilled).

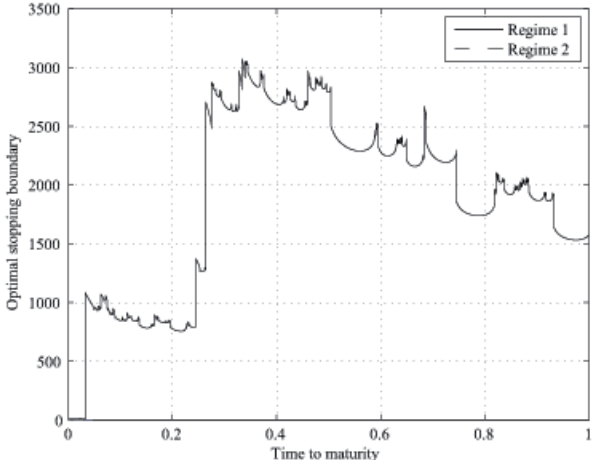

Figure 3.9: Optimal stopping boundary for regime 1 and regime 2 (stability condition is broken).

square error (RMSE) for the problem of two regime switching model with parameters (3.77).

In accordance with [70] the reference value $u^{*}\left(x_{j}, T\right)$ is chosen to be the solution by the binomial tree method of Liu with 1000 steps.

In order to compute convergence rate in space approximate solutions are calculated for different step sizes $h$ using a fixed time step $k$. In Table 3.8 the results are presented. Time step $k$ is chosen to guarantee stability for all tested space steps $h$. The convergence rate in space $\gamma_{h}$ is calculated as the mean value of all combinations of $h_{1}$ and $h_{2}$ :

$$
\gamma_{h}=1.86
$$

Analogous procedure is done for fixed $h=10^{-2}$ and various space steps $k$. The results are collected in the Table 3.9. Computational time is linearly increasing with growth of the number of time levels. The average RMSE is proportional to the time step. Using the formula (1.21) in terms of time steps and taking the mean value of all combinations, one gets the following convergence rate in time:

$$
\gamma_{k}=1.15
$$

Numerical results and CPU time for two-regime model with the parameters (3.77) computed by a fully implicit fitted finite volume (IFV) method based on 


\section{FRONT-FIXING METHOD FOR SOME ADVANCED MODELS}

\begin{tabular}{|c|ccc|}
\hline Method & Regime 1 & Regime 2 & CPU time, sec. \\
\hline & \multicolumn{3}{|c}{$h=10^{-1}, k=10^{-2}$} \\
Explicit & 1.9543 & 1.8636 & 0.1248 \\
Crank-Nicolson & 1.9756 & 1.8863 & 0.1248 \\
Fully implicit & 1.9956 & 1.9073 & 0.1092 \\
\hline & $h=10^{-2}$, & $k_{\text {expl }}=10^{-4}$, & $k_{\text {impl }}=10^{-2}$ \\
Explicit & 1.9713 & 1.8818 & 4.9140 \\
Crank-Nicolson & 1.9720 & 1.8824 & 49.2004 \\
Fully implicit & 1.9712 & 1.8817 & 34.8817 \\
\hline
\end{tabular}

Table 3.7: Comparison of explicit and implicit methods. Time step of explicit and implicit methods are denoted correspondingly by $k_{e x p l}$ and $k_{i m p l}$.

\begin{tabular}{|c|c|c|c|c|}
\hline $\mathrm{h}$ & 0.08 & 0.04 & 0.02 & 0.01 \\
\hline Regime 1 & $2.664 \mathrm{e}-2$ & $5.601 \mathrm{e}-3$ & $1.489 \mathrm{e}-3$ & $8.669 \mathrm{e}-4$ \\
Regime 2 & $3.216 \mathrm{e}-2$ & $8.729 \mathrm{e}-3$ & $1.955 \mathrm{e}-3$ & $1.742 \mathrm{e}-4$ \\
Average & $2.939 \mathrm{e}-2$ & $7.165 \mathrm{e}-3$ & $1722 \mathrm{e}-3$ & $5.206 \mathrm{e}-4$ \\
\hline CPU time, sec. & 1.2948 & 1.4976 & 1.7472 & 2.5584 \\
\hline
\end{tabular}

Table 3.8: RMSE and computational time for fixed $k=10^{-4}$ and various $h$.

penalty approach are available in [116]. Table 3.10 shows the error of both frontfixing (FF) and IFV methods for both regimes on different meshes with respect to the binomial tree method in Table 1 of [71] as well as computational time. This fact proves the efficiency of the proposed method.

Example 3.2.3. Recently authors in [5] compare iterated optimal stopping (IOS) and local policy iteration (LPI) methods for regime-switching model with the parameters:

$$
\boldsymbol{r}=\left(\begin{array}{l}
0.05 \\
0.05
\end{array}\right), \boldsymbol{\sigma}=\left(\begin{array}{l}
0.3 \\
0.4
\end{array}\right), Q=\left(\begin{array}{rr}
-3 & 3 \\
2 & -2
\end{array}\right), T=1, E=10
$$

Numerical solutions provided by both IOS and LPI methods for data (3.78) are presented in [5] showing that prices grow as the step sizes are refined. For the 


\begin{tabular}{|c|c|c|c|c|}
\hline $\mathrm{k}$ & $10^{-4}$ & $5 \cdot 10^{-5}$ & $2.5 \cdot 10^{-5}$ & $1.25 \cdot 10^{-5}$ \\
\hline Regime 1 & $8.669 \mathrm{e}-4$ & $4.163 \mathrm{e}-4$ & $2.168 \mathrm{e}-4$ & $1.234 \mathrm{e}-4$ \\
Regime 2 & $1.742 \mathrm{e}-4$ & $1.009 \mathrm{e}-4$ & $2.885 \mathrm{e}-5$ & $7.973 \mathrm{e}-6$ \\
Average & $5.206 \mathrm{e}-4$ & $2.586 \mathrm{e}-4$ & $1.228 \mathrm{e}-4$ & $6.569 \mathrm{e}-5$ \\
\hline CPU time, sec. & 2.5584 & 4.5708 & 8.9389 & 17.9870 \\
\hline
\end{tabular}

Table 3.9: RMSE and computational time for fixed $h=10^{-2}$ and various $k$.

\begin{tabular}{|c|c|c|}
\hline & IFV, $1601 \times 1281$ & FF, $300 \times 4 \cdot 10^{4}$ \\
\hline Error (regime 1) & $2.00 \mathrm{e}-4$ & $2.94 \mathrm{e}-4$ \\
Error (regime 2) & $6.00 \mathrm{e}-4$ & $4.89 \mathrm{e}-5$ \\
\hline CPU-time, sec. & 34.96 & 8.94 \\
\hline
\end{tabular}

Table 3.10: Comparison of the efficiency of the IFV and proposed method (FF).

highest refinement the values are as follows

$$
\begin{array}{ll}
\text { IOS: } & 1.174888119, \\
\text { LPI: } & 1.174888084 .
\end{array}
$$

Table 3.11 reveals the oncoming of our results to the values (3.79) as time step decreases and space step is fixed including CPU-time.

As the study of the Greeks is an important issue in option pricing because they show relevant properties of the price (see in [58], chapter 14), in Fig. 3.10 and 3.11 we focus in particular on two of the most used ones. The Delta and Gamma of the option are presented at holding region for both regimes of option with parameters (3.78) showing similar behaviour of Greeks as in [116].

In the last example we apply the proposed method to the four-regime case. Numerical option values and optimal stopping boundaries are presented as well as comparison with efficient recent results given in [70].

Example 3.2.4. The four-regime option is considered. The model parameters are 


\section{FRONT-FIXING METHOD FOR SOME ADVANCED MODELS}

\begin{tabular}{|c|c|c|}
\hline$\mu$ & Value & CPU-time, sec. \\
\hline 1.56 & 1.1743801593 & 2.23 \\
\hline 0.6 & 1.1748081977 & 3.88 \\
\hline 0.5 & 1.1748742268 & 4.69 \\
\hline 0.46 & 1.1748890632 & 4.94 \\
\hline
\end{tabular}

Table 3.11: Option values at $S=10.0$ in Regime 1 for various mesh ratios $\mu=\frac{k}{h^{2}}$ and spatial step $h=10^{-2}$.

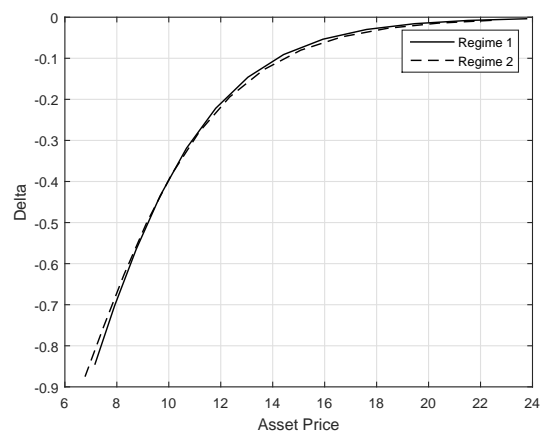

Figure 3.10: Delta of option with parameters 3.78 in both regimes.

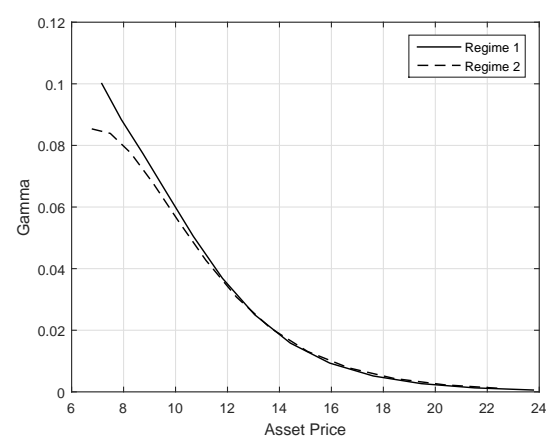

Figure 3.11: Gamma of option with parameters $(3.78)$ in both regimes.

chosen as

$$
\boldsymbol{r}=\left(\begin{array}{l}
0.02 \\
0.10 \\
0.06 \\
0.15
\end{array}\right), \boldsymbol{\sigma}=\left(\begin{array}{l}
0.9 \\
0.5 \\
0.7 \\
0.2
\end{array}\right), Q=\left(\begin{array}{rrrr}
-1 & \frac{1}{3} & \frac{1}{3} & \frac{1}{3} \\
\frac{1}{3} & -1 & \frac{1}{3} & \frac{1}{3} \\
\frac{1}{3} & \frac{1}{3} & -1 & \frac{1}{3} \\
\frac{1}{3} & \frac{1}{3} & \frac{1}{3} & -1
\end{array}\right), T=1, E=9
$$

The numerical domain is truncated at the point $x_{\max }=3$, step sizes are as in Example $1, h=10^{-2}, k=10^{-4}$. The option price for every regime and optimal stopping boundaries are presented on the Figures 3.12 and 3.13 .

Comparison with penalty method [70] and tree method is presented in Table 3.12 by computing the numerical solution at several values of asset price $S$. It is shown how close the results are.

The results of this section have been published in [43]. 


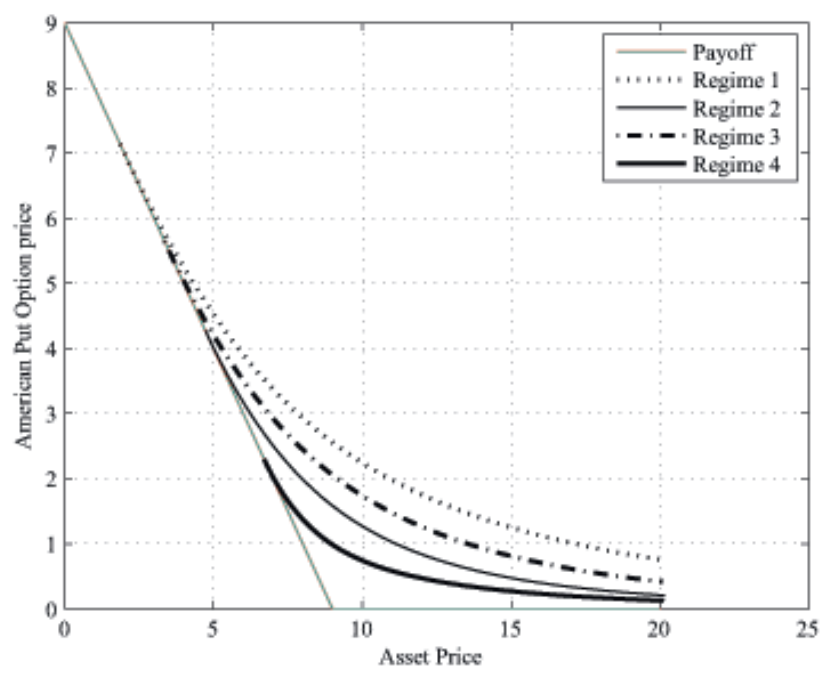

Figure 3.12: American put option price curves at $\tau=T$ for the four regime model and its payoff.

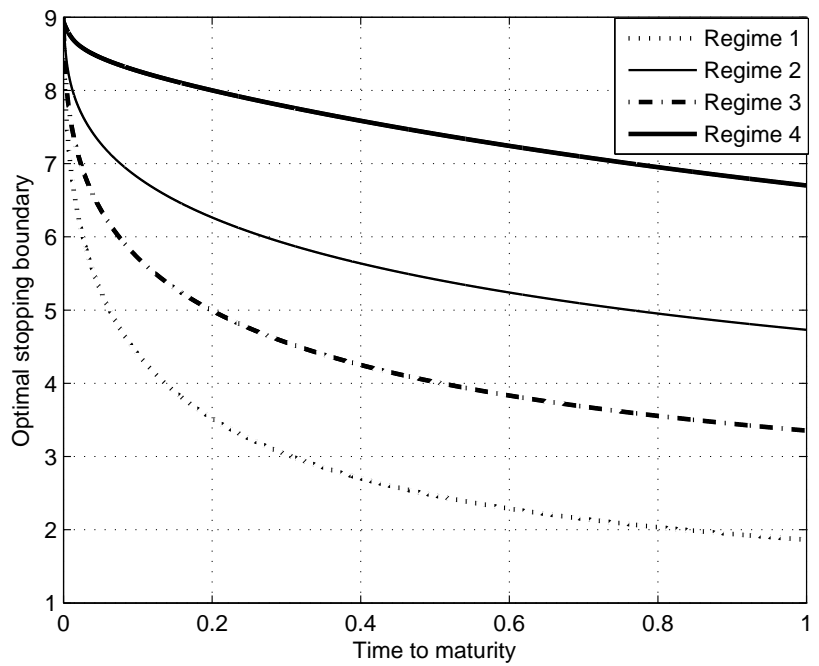

Figure 3.13: Optimal stopping boundary for the four regime American put option with parameters 3.80 ). 


\begin{tabular}{|c|c|c|c|c|c|}
\hline Regime & Method & $S=7.5$ & $S=9.0$ & $S=10.5$ & $S=12.0$ \\
\hline 1 & FF-expl & 3.1421 & 2.5563 & 2.1047 & 1.7524 \\
& ETD-CN & 3.1513 & 2.5641 & 2.1113 & 1.7578 \\
& Tree & 3.1433 & 2.5576 & 2.1064 & 1.7545 \\
\hline 2 & FF-expl & 2.2313 & 1.5827 & 1.1406 & 0.8368 \\
& ETD-CN & 2.2384 & 1.5884 & 1.1451 & 0.8404 \\
& Tree & 2.2319 & 1.5834 & 1.1417 & 0.8377 \\
\hline 3 & FF-expl & 2.6739 & 2.0559 & 1.6004 & 1.2614 \\
& ETD-CN & 2.6813 & 2.0623 & 1.6057 & 1.2658 \\
& Tree & 2.6746 & 2.0568 & 1.6014 & 1.2625 \\
\hline 4 & FF-expl & 1.6573 & 0.9850 & 0.6546 & 0.4700 \\
& ETD-CN & 1.6664 & 0.9903 & 0.6580 & 0.4725 \\
& Tree & 1.6574 & 0.9855 & 0.6553 & 0.4708 \\
\hline
\end{tabular}

Table 3.12: Comparison of American put option prices in a four-regime model 


\section{Behavioural modelling of option pricing}

American option gives the right to the owner to exercise it and receive the corresponding payoff. Irrational behaviour is frequent in market trading due to many reasons such as emotional reactions or unperfect information ([36], [95] and [50]). Empirical studies illustrate a lot of situations when irrational exercise takes place. For example, in [36] the irrational exercise of S \& P 100 call and put options is illustrated, while [95] shows that the clients of discount brokers irrationally exercise their calls too early, mainly in the case of less sophisticated investors. Sometimes, the irrational exercise is related rely on specific circumstances around the global investment position, for example when the American option is part of an hedging strategy in which the optimal exercise rule results to be not optimal.

Recently, in [50] a new nonlinear Black-Scholes model that takes into account irrational exercise behaviour is proposed. More precisely, the authors characterize the rationality of exercise of an American put option in terms of a rationality parameter by means of an intensity based model for the valuation of the American puts, so that the exercise decision occurs as the the first jump time of a process with stochastic intensity. Thus, the exercise intensity depends on how profitable is to exercise, i.e. the larger the difference between the payoff and the value of the American put if not exercised the greater the exercise intensity. Thus, the rationality parameter accounts for the dependence of the exercise intensity in terms 


\section{BEHAVIOURAL MODELLING OF OPTION PRICING}

of the profitability. In [50], the authors provide a probabilistic proof of the existence of solution as well as of the convergence of the model to the solution of the American put option in the rational case when the rational parameter tends to infinity. Moreover, by using Feynmann-Kac theorem, the associated nonlinear Black-Scholes model is posed.

In the present chapter we confirm numerically that the solution of the irrational problem proposed in [50] for large values of rationality parameter tends to the solution of the rational American option problem. This technique has been successfully applied to a regime switching model described in previous chapter.

We address the numerical approach of the solution of the nonlinear model for a vanilla American put proposed in [50] as well as for a regime switching model. In both cases we introduce a new boundary condition when the price of the underlying asset tends to zero. Note that the classical boundary condition for zero underlying price of the rational American option does not apply in the irrational case. Appropriate boundary conditions are specially required for the localization procedure to confine the problem in a bounded domain as previous step to the use of numerical techniques. Apart from the intensity functions proposed in [50], we introduce two additional smooth intensity functions.

A proposed numerical solution is based on a $\theta$-method for PDE discretization. A transformation technique is used in order to take benefit of some numerical advantages. In fact, the PDE problem is transformed into a problem with constant coefficients in diffusion, convection and reaction parts of the equation together with a nonlinear term involving the intensity function. Positivity, stability and consistency of the proposed methods are studied.

After addressing the stability analysis, some numerical examples illustrate the expected order of convergence for the classical explicit, fully implicit and CrankNicolson particular cases of the $\theta$-method.

As departure point we assume the standard conditions of Black-Scholes model and consider a risky asset, the price of which follows a geometric brownian motion under risk neutral probability, so that the price at time $t, S_{t}$, satisfies the following stochastic differential equation

$$
d S_{t}=r S_{t} d t+\sigma d W_{t}, \quad S_{0} \quad \text { given, }
$$

where $r$ denotes the constant risk-free interest rate, $\sigma$ is the constant instantaneous volatility of the asset and $W_{t}$ represents a standard Brownian motion. If we consider an American put option with strike price $E$ and maturity $T$ on the previous asset, 
the exercise value at time $t<T$ is given by $\left(E-S_{t}\right)^{+}$, so that the arbitrage-free value of the American put, $P_{t}^{A}=P^{A}\left(t, S_{t}\right)$, can be characterized as the solution of an optimal stopping time problem

$$
P_{t}^{A}=P^{A}\left(t, S_{t}\right)=\sup _{t \leq \tau \leq T} E_{t}\left[\exp (-r(\tau-t))\left(E-S_{\tau}\right)^{+}\right]
$$

where $E_{t}$ denotes the conditional expectation to time $t$ in the risk-neutral probability measure. Thus, there exists an optimal stopping time, $\tau^{*}$, for which the supremum is attained.

In [50], in order to model irrational exercise, the authors introduce the irrational exercise rule, $\tau^{*}$, as the minimum of the terminal time, $T$, and the first jump time of a point process with stochastic intensity $\left(\mu_{t}\right)_{0 \leq t \leq T}$ (see [15], for example). Next, in terms of this family of intensity functions, a strictly positive rationality parameter, $\lambda$, measuring the rationality of the behaviour of the American option owner, is introduced. Moreover, if $\lambda$ is the parameter of the family of intensity functions, $f^{\lambda}$, and we denote by $\tau^{*}(\lambda)$ the associated exercise strategies, when $\lambda$ tends to infinity we recover the arbitrage free price of the American put (i.e. the one associated to a fully rational exercise). If the exercise policy depends on how profitable is to exercise, then the relation between profitability and stochastic exercise intensity can be written in the form:

$$
\mu_{t}=f\left(\left(E-S_{t}\right)-P\left(t, S_{t} ; \tau^{*}\right)\right)
$$

where $f:[-K, K] \rightarrow[0,+\infty)$ denotes the intensity function and $\tau^{*}$ is the exercise strategy. In Theorem 2 in [50], sufficient conditions for an index of a family of intensity functions to be a rationality parameter are stated. Hereafter we recall the theorem.

Theorem 4.0.3. Let $\left(f^{\lambda}\right)_{\lambda>0}$ be a family of positive deterministic intensity functions. For each $\lambda>0$, let the stochastic intensity process be given by

$$
\left.\mu_{t}^{\lambda}=f^{\lambda}\left(\left(E-S_{t}\right)^{+}-P^{\lambda}\left(t, S_{t}\right)\right)\right)
$$

where $P^{\lambda}\left(t, S_{t}\right)=P^{\lambda}\left(t, S_{t} ; \tau^{*}(\lambda)\right.$ and $\tau^{*}(\lambda)$ is the exercise strategy of the American put given as the first jump time of a point process with intensity $\mu^{\lambda}$. Let $\nu_{\lambda}(x)=1_{(x<0)} \sup _{y \leq x} f^{\lambda}(y)+1_{(x \geq 0)} \sup _{y \geq x} f^{\lambda}(y)$ and assume that

- $\nu_{\lambda}(0+) \rightarrow \infty$ as $\lambda \rightarrow \infty$. 
- There exists a function $\epsilon:(0, \infty) \rightarrow(0, \infty)$ such that $\left.\nu_{\lambda}(-\epsilon(\lambda))\right) \rightarrow 0$ and $\epsilon(\lambda) \nu_{\lambda}(0-) \rightarrow 0$ as $\lambda \rightarrow \infty$.

Then $\lambda$ is a rationality parameter in the sense that for every $t \in[0, T]$ we have that $P^{\lambda}\left(t, S_{t}\right)$ tends to $P^{A}\left(t, S_{t}\right)$ when $\lambda \rightarrow \infty$. Moreover, if $f^{\lambda}$ is increasing then $f^{\lambda}=\nu_{\lambda}$.

Note that in the previous framework, the family of intensity functions $f^{\lambda}$ has to be chosen so that $\lambda$ satisfies the condition of rationality parameter. We first consider the two cases proposed in [50] and we additionally propose two alternative expressions.

The first choice is given by

$$
f_{1}^{\lambda}(x)= \begin{cases}\lambda, & \text { for } x \geq 0 \\ 0, & \text { for } x<0\end{cases}
$$

while the second is

$$
f_{2}^{\lambda}(x)=\lambda e^{\lambda^{2} x}
$$

Remark. In the first case, the family of intensity functions is related to the application of the penalty method to the classical American put option problem. Using this family it is certain that the buyer does not exercise when it is not profitable. The second specification (4.5) allows that the buyer is not just affected by whether it is profitable or not to exercise, but also by the amount of profit [50].

The proposed intensity functions are the smooth analogue of stepwise function (4.4):

$$
\begin{gathered}
f_{3}^{\lambda}(x)=\frac{2 \lambda}{1+e^{-\lambda^{2} x}}, \\
f_{4}^{\lambda}(x)=\lambda\left(1+\frac{2}{\pi} \arctan \lambda^{2} x\right) .
\end{gathered}
$$

From the mathematical point of view, note that in the first case the functions are discontinuous at zero, while in the second as well as in the case of new proposed functions all derivatives are available at any point. 


\subsection{Pricing of American option with rationality para-}

\section{meter}

After stating some sufficient conditions to define a rationality parameter, several steps are required to establish the nonlinear PDE model. If we consider the irrational exercise rule, $\tau^{*}$, as the first jump time of a point precess with stochastic intensity $\mu_{t}=\alpha\left(S_{t}, t\right), \alpha$ being a positive deterministic measurable function, then the American put value is given by expression

$$
\begin{aligned}
P\left(S_{t}, t\right) & =\exp (-r(T-t)) E\left[\left(E-S_{t}\right)^{+} 1_{\left(\tau^{*} \geq T\right)}\right] \\
& +E_{t}\left[\exp \left(-r\left(\tau^{*}-t\right)\right)\left(E-S_{\tau^{*}}\right)^{+} 1_{\left(\tau^{*}<T\right)}\right] .
\end{aligned}
$$

Next, by using Proposition 3.1 in [80], previous expression can be written in the equivalent form

$$
\begin{aligned}
& P\left(S_{t}, t\right)=\exp (-r(T-t)) E_{t}\left[\exp \left(-\int_{t}^{T} \alpha\left(u, S_{u}\right) d u\right)\left(E-S_{T}\right)^{+}\right] \\
& +\int_{t}^{T} \exp (-r(u-t)) E_{t}\left[\alpha\left(u, S_{u}\right) \exp \left(\int_{t}^{u} \alpha\left(v, S_{v}\right) d v\right)\left(E-S_{u}\right)^{+}\right] d u .
\end{aligned}
$$

For $f:[-K, K] \rightarrow[0, \infty)$, we can define $\alpha(t, s)=f\left((E-s)^{+}-P(t, s)\right)$, apply Feynman-Kac [68] and (in certain abuse of notation) consider $\tau=T-t$ as time to maturity to obtain the following nonlinear Black-Scholes equation in the unbounded domain $\Omega=(0,+\infty) \times(0, T)$ :

$$
\frac{\partial P}{\partial \tau}=\frac{\sigma^{2}}{2} S^{2} \frac{\partial^{2} P}{\partial S^{2}}+(r-q) S \frac{\partial P}{\partial S}-r P+f\left((E-S)^{+}-P\right)\left((E-S)^{+}-P\right),
$$

jointly with the initial condition (provided by the put option payoff at maturity):

$$
P(S, 0)=(E-S)^{+} .
$$

Having in view a localization procedure to approximate the previous problem by a problem posed in a bounded domain, next we consider the behaviour of the American put option in the irrational exercise setting when $S \rightarrow 0$ or $S \rightarrow \infty$. When $S \rightarrow \infty$, clearly the usual condition as in the rational case is maintained, so that

$$
\lim _{S \rightarrow \infty} P(S, \tau)=0 .
$$


However, when $S \rightarrow 0$, the standard condition for American options, $P(0, \tau)=$ $E$, is no longer valid in the irrational case, as prices bellow exercise price may occur due to irrational exercise, which is more evident when the rationality parameter tends to zero. The typical boundary condition for European options $P(0, \tau)=$ $E e^{-r \tau}$ is not consistent with the equation for $\lambda \rightarrow-\infty$, as the solution converges to the one of the rational case of American options. Since equation (4.8) is nonlinear and describes option pricing with rationality parameter, a new boundary condition has to be established. Therefore, we propose to pass to the limit in equation (4.8) when $S \rightarrow 0$ :

$$
\frac{\partial P}{\partial \tau}(0, \tau)=-r P(0, \tau)+f(E-P(0, \tau))(E-P(0, \tau)) .
$$

The previous equation allows to adapt the option price when $S=0$ according to rationality of the holder.

For simplicity, let us consider the equivalent dimensionless formulation of the problem:

$$
\frac{\partial u}{\partial \tau}=\frac{\sigma^{2}}{2} x^{2} \frac{\partial^{2} u}{\partial x^{2}}+(r-q) x \frac{\partial u}{\partial x}-r u+f\left((1-x)^{+}-u\right)\left((1-x)^{+}-u\right)
$$

with the initial condition

$$
u(0, x)=(1-x)^{+}
$$

with boundary conditions

$$
\begin{gathered}
\lim _{x \rightarrow \infty} u(x, \tau)=0 \\
\frac{\partial u}{\partial \tau}(0, \tau)=-r u(0, \tau)+f(1-u(0, \tau))(1-u(0, \tau)) .
\end{gathered}
$$

Initial values are found from the following formula

$$
P(S, \tau)=E u(x, \tau), \quad S=E x .
$$

It is well known that a closed form solution for the classical American option pricing problem with rational exercise cannot be obtained, so that a wide variety of numerical methods to approximate the option price have been proposed in the literature. In the irrational exercise case, the nonlinear Black-Scholes problem defined by equations (4.11)-(4.14) is posed, so that the analytical expression of the solution for a general intensity function $f$ is not available and we also require the use of numerical methods. 


\subsubsection{Numerical solution with irrational exercise}

In the present section, we propose finite differences techniques for the discretization of the problem defined by equations (4.11) - 4.14). As the problem is initially posed in an unbounded domain, a first step is to approximate it by a bounded domain with appropriate boundary conditions, so that this localization does not affect the numerical solution in the region of financial interest. Following the ideas in [66] for European vanilla options, we truncate the domain in the original financial variables by using the finite upper value $S_{\max }=3 E$ in the asset direction. Thus, the computational domain in the new variables for the problem (4.11)-(4.12) is $[0, T] \times[0,3]$ equipped with a uniform grid with constant step sizes $\Delta s=\frac{3}{M}$ and $\Delta \tau=\frac{T}{N}$ in the "space" and time directions respectively. Nodes of the grid are denoted as follows

$$
s_{j}=j \Delta s, 0 \leq j \leq M ; \quad \tau^{n}=n \Delta \tau, 0 \leq n \leq N .
$$

Let us denote $u_{j}^{n} \approx u\left(s_{j}, \tau^{n}\right)$, then in terms of the parameter $\theta \in[0,1]$, the so called $\theta$-method can be written in the form

$$
\begin{aligned}
\frac{u_{j}^{n+1}-u_{j}^{n}}{\Delta \tau}= & \frac{\sigma^{2}}{2} s_{j}^{2}\left[\theta \frac{u_{j+1}^{n}-2 u_{j}^{n}+u_{j-1}^{n}}{\Delta s^{2}}+(1-\theta) \frac{u_{j+1}^{n+1}-2 u_{j}^{n+1}+u_{j-1}^{n+1}}{\Delta s^{2}}\right]+ \\
& (r-q) s_{j}\left[\theta \frac{u_{j+1}^{n}-u_{j-1}^{n}}{2 \Delta s}+(1-\theta) \frac{u_{j+1}^{n+1}-u_{j-1}^{n+1}}{2 \Delta s}\right]- \\
& r\left[\theta u_{j}^{n}+(1-\theta) u_{j}^{n+1}\right]+\left[\theta f_{j}^{n}+(1-\theta) f_{j}^{n+1}\right], \\
& 1 \leq j \leq M-1, \quad 0 \leq n \leq N-1,
\end{aligned}
$$

where $f_{j}^{n}=f\left(\alpha_{j}^{n}\right) \alpha_{j}^{n}$, with

$$
\alpha_{j}^{n}=\left(1-s_{j}\right)^{+}-u_{j}^{n} .
$$

Note that for $\theta=1$ the scheme (4.15) corresponds to the explicit one, for $\theta=0.5$ it is known as Crank-Nicolson scheme and for $\theta=0$ the scheme becomes the fully implicit one.

The boundary condition (4.14) is approximated by a forward in time difference. Therefore, the values at the boundary points at each time step are computed as follows

$$
\begin{aligned}
& u_{M}^{n+1}=0, \\
& u_{0}^{n+1}=(1-r \Delta \tau) u_{0}^{n}-\Delta \tau f_{0}^{n} .
\end{aligned}
$$


The system of equations 4.15 for $j=1, \ldots, M-1$ jointly with boundary values 4.16)-(4.17) is a closed nonlinear system. We propose the use of Newton's method to solve it numerically. For simplicity, we present the scheme 4.15) by using a vector form. Thus, let us introduce the following vectors of the coefficients, $j=1, \ldots, M-1$ :

$$
\begin{aligned}
& a_{j}=(1-\theta)\left[(r-q) s_{j} \frac{\Delta \tau}{2 \Delta s}-\frac{\sigma^{2}}{2} s_{j}^{2} \frac{\Delta \tau}{\Delta s^{2}}\right], \\
& b_{j}=1+(1-\theta)\left[\sigma^{2} s_{j}^{2} \frac{\Delta \tau}{\Delta s^{2}}+r \Delta \tau\right], \\
& c_{j}=-(1-\theta)\left[(r-q) s_{j} \frac{\Delta \tau}{2 \Delta s}+\frac{\sigma^{2}}{2} s_{j}^{2} \frac{\Delta \tau}{\Delta s^{2}}\right] \\
& \tilde{a}_{j}=-\theta\left[(r-q) s_{j} \frac{\Delta \tau}{2 \Delta s}-\frac{\sigma^{2}}{2} s_{j}^{2} \frac{\Delta \tau}{\Delta s^{2}}\right], \\
& \tilde{b}_{j}=1-\theta\left[\sigma^{2} s_{j}^{2} \frac{\Delta \tau}{\Delta s^{2}}+r \Delta \tau\right] \\
& \tilde{c}_{j}=\theta\left[(r-q) s_{j} \frac{\Delta \tau}{2 \Delta s}+\frac{\sigma^{2}}{2} s_{j}^{2} \frac{\Delta \tau}{\Delta s^{2}}\right] .
\end{aligned}
$$

Then, the scheme 4.15) can be presented in the following vector form:

$$
A U^{n+1}-\Delta \tau(1-\theta) F\left(U^{n+1}\right)=B U^{n}+\Delta \tau \theta F\left(U^{n}\right)+\left(\tilde{a}_{1} u_{0}^{n}-a_{1} u_{0}^{n+1}\right) I_{1},
$$

where $A$ and $B$ are the tridiagonal matrices with coefficients:

$$
\begin{aligned}
A & =\left(\begin{array}{ccccccc}
b_{1} & c_{1} & 0 & 0 & \ldots & 0 & 0 \\
a_{2} & b_{2} & c_{2} & 0 & \ldots & 0 & 0 \\
\ddots & \ddots & \ddots & \ddots & \ddots & \ddots & \ddots \\
0 & 0 & 0 & 0 & \ldots & a_{M-1} & b_{M-1}
\end{array}\right), \\
B & =\left(\begin{array}{ccccccc}
\tilde{b}_{1} & \tilde{c}_{1} & 0 & 0 & \ldots & 0 & 0 \\
\tilde{a}_{2} & \tilde{b}_{2} & \tilde{c}_{2} & 0 & \ldots & 0 & 0 \\
\ddots & \ddots & \ddots & \ddots & \ddots & \ddots & \ddots \\
0 & 0 & 0 & 0 & \ldots & \tilde{a}_{M-1} & \tilde{b}_{M-1}
\end{array}\right)
\end{aligned}
$$

and we use the column vectors $U^{n}=\left\{u_{j}^{n}\right\}, F\left(U^{n}\right)=\left\{f_{j}^{n}\right\}, I_{1}=[1,0, \ldots, 0]$.

Vector equation 4.18) can be rewritten in the form $G=0$, where the vector $G$ is the difference between left and right hand sides of 4.18. 


\subsection{Pricing of American option with rationality parameter}

\section{Newton's method for the nonlinear fully discretized problem}

At each time step of the finite differences algorithm we have to compute $U^{n+1}$, as the solution of nonlinear system:

$G\left(U^{n+1}\right)=A U^{n+1}-\Delta \tau(1-\theta) F\left(U^{n+1}\right)-B U^{n}-\Delta \tau \theta F\left(U^{n}\right)-\left(\tilde{a}_{1} u_{0}^{n}-a_{1} u_{0}^{n+1}\right) I_{1}=0$.

We approximate the solution by using Newton's iterative method. As initial guess, we take the numerical solution computed at the previous time level, i.e. $U_{0}^{n+1}=$ $U^{n}$. Then, at step $i$ of Newton algorithm, for a previously computed value of $U_{i}^{n+1}$, the values of $G\left(U_{i}^{n+1}\right)$ and its Jacobian, $J_{G}\left(U_{i}^{n+1}\right)$, have to be computed.

Note that from (4.18) the Jacobian is given by

$$
\left.J_{G}\left(U_{i}^{n+1}\right)=A-\Delta \tau(1-\theta) J_{F}\left(U_{i}^{n+1}\right)\right) .
$$

Since $A$ is the matrix of constant components, the main difficulty is computation of Jacobian $J_{F}\left(U_{i}^{n+1}\right)$. Note that when function $f$ belongs to the family given by (4.4), it is not differentiable at the point $\alpha=0$. We suppose that the function has finite derivative at this point, therefore the diagonal components of Jacobian $J_{F}\left(U_{i}^{n+1}\right)$ take form for every component $j=1, \ldots, M-1$

$$
J_{F}\left(U_{i}^{n+1}\right)_{j, j}=\frac{\partial F_{j}}{\partial u_{j}}=-f^{\prime}\left(\alpha_{j}^{n+1}\right) \alpha_{j}^{n+1}-f\left(\alpha_{j}^{n+1}\right)=-f\left(\alpha_{j}^{n+1}\right),
$$

since at the point $\alpha=0$ the derivative is finite, but multiplied by $\alpha$, and the derivative $f^{\prime}(\alpha)=0$ for all $\alpha \neq 0$. $U_{i}^{n+1}$.

Note that here $\alpha_{j}=\left(1-s_{j}\right)^{+}-u_{j}^{n+1}$, where $u_{j}^{n+1}$ is $j$-th component of vector

If the function $f$ belongs to the family (4.5), then the Jacobian is a diagonal matrix of elements:

$$
J_{F}\left(U_{i}^{n+1}\right)_{j, j}=-f\left(\alpha_{j}^{n+1}\right)\left[\lambda^{2} \alpha_{j}^{n+1}+1\right] .
$$

If the function $f$ belongs to the family $(4.6)$, then

$$
J_{F}\left(U_{i}^{n+1}\right)_{j, j}=-f\left(\alpha_{j}^{n+1}\right)\left[1+\lambda^{2} \alpha_{j}^{n+1} \frac{e^{-\lambda^{2} \alpha_{j}^{n+1}}}{1+e^{-\lambda^{2} \alpha_{j}^{n+1}}}\right] .
$$

Finally, if function $f$ is described by expression (4.7), then the Jacobian takes the following form

$$
J_{F}\left(U_{i}^{n+1}\right)_{j, j}=-f\left(\alpha_{j}^{n+1}\right)-\frac{2 \lambda^{3} \alpha_{j}^{n+1}}{\pi\left(1+\left(\lambda^{2} \alpha_{j}^{n+1}\right)^{2}\right)} .
$$


In order to obtain the next iteration the following linear system has to be solved:

$$
J_{G}\left(U_{i}^{n+1}\right)\left(U_{i+1}^{n+1}-U_{i}^{n+1}\right)=-G\left(U_{i}^{n+1}\right) .
$$

Since $J_{G}\left(U_{i}^{n+1}\right)$ is tridiagonal matrix, Thomas algorithm is used without storing the full matrix $J_{G}\left(U_{i}^{n+1}\right)$ but only its nonzero components in three vectors. The algorithm stops when norm of increment $\Delta U_{i+1}^{n+1}=U_{i+1}^{n+1}-U_{i}^{n+1}$ is below a certain prescribed tolerance.

The qualitative properties of the scheme can be improved by transforming the original equation. In the following section we propose logarithmic transformation that allows to construct numerical scheme with constant coefficients.

\subsubsection{Transformation and explicit finite difference method}

We introduce new variable

$$
x=\ln \frac{S}{E} .
$$

Then the original problem is transformed to the following for $x \in \mathbb{R}$ :

$\frac{\partial u}{\partial \tau}=\frac{\sigma^{2}}{2} \frac{\partial^{2} u}{\partial x^{2}}+\left(r-q-\frac{\sigma^{2}}{2}\right) \frac{\partial u}{\partial x}-r u+f\left(E\left(1-e^{x}\right)^{+}-E u\right)\left(\left(1-e^{x}\right)^{+}-u\right)$

with the initial condition

$$
u(0, x)=\left(1-e^{x}\right)^{+}
$$

with boundary conditions

$$
\begin{gathered}
\lim _{x \rightarrow \infty} u(x, t)=0 \\
\lim _{x \rightarrow-\infty} \frac{\partial u}{\partial \tau}(\tau, x)=\lim _{x \rightarrow-\infty}(-r u(\tau, x)+f(E(1-u(\tau, x)))(1-u(\tau, x))) .
\end{gathered}
$$

Initial values are found from the following formula

$$
P(\tau, S)=E u(\tau, x), \quad S=E e^{x} .
$$

Numerical solution has to be found on unbounded domain $(-\infty, \infty) \times[0, T]$. Computational domain is truncated as $\left[x_{\min }, x_{\max }\right] \times[0, T]$. Let us introduce uniform grid $\left\{x_{j}, \tau^{n}\right\}$ with steps $h=\frac{x_{\max }-x_{\min }}{N_{x}}$ and $k=\frac{T}{N_{\tau}}$ for chosen $N_{x}$ and $N_{\tau}$ correspondingly such that

$$
\begin{array}{r}
x_{j}=x_{\min }+j h, \quad j=0, \ldots, N_{x}, \\
\tau^{n}=n k, \quad n=1, \ldots, N_{\tau} .
\end{array}
$$


Let us denote $u_{j}^{n} \approx u\left(x_{j}, \tau^{n}\right)$, then explicit finite difference scheme can be written in the form

$$
\begin{aligned}
\frac{u_{j}^{n+1}-u_{j}^{n}}{k}= & \frac{\sigma^{2}}{2} \frac{u_{j+1}^{n}-2 u_{j}^{n}+u_{j-1}^{n}}{h^{2}}+\left(r-q-\frac{\sigma^{2}}{2}\right) \frac{u_{j+1}^{n}-u_{j-1}^{n}}{2 h}- \\
& r u_{j}^{n}+f_{j}^{n} \alpha_{j}^{n}, \quad 1 \leq j \leq N_{x}-1, \quad 0 \leq n \leq N_{\tau}-1,
\end{aligned}
$$

where $f_{j}^{n}=f\left(E \alpha_{j}^{n}\right) \alpha_{j}^{n}$ and $\alpha_{j}^{n}=\left(1-e^{x_{j}}\right)^{+}-u_{j}^{n}$.

The boundary condition (4.22) is approximated by a forward in time difference. Therefore, the values at the boundary points at each time step are computed as follows

$$
\begin{aligned}
& u_{N_{x}}^{n+1}=0, \\
& u_{0}^{n+1}=(1-r k) u_{0}^{n}+k f_{0}^{n} .
\end{aligned}
$$

Formula (4.23) for $j=1, \ldots, N_{x}-1$ jointly with boundary values (4.24)(4.25) define the proposed explicit scheme. Next, let us introduce the following coefficients

$$
\begin{aligned}
& b_{1}=\frac{\sigma^{2}}{2} \frac{k}{h^{2}}-\left(r-q-\frac{\sigma^{2}}{2}\right) \frac{k}{2 h}, \\
& b_{2}=1-\left(\sigma^{2} \frac{k}{h^{2}}+r k\right), \\
& b_{3}=\frac{\sigma^{2}}{2} \frac{k}{h^{2}}+\left(r-q-\frac{\sigma^{2}}{2}\right) \frac{k}{2 h} .
\end{aligned}
$$

Then, formula (4.23) can be presented in the following form:

$$
u_{j}^{n+1}=b_{1} u_{j-1}^{n}+b_{2} u_{j}^{n}+b_{3} u_{j+1}^{n}+k f_{j}^{n}, \quad j=1, \ldots, N_{x}-1 .
$$

In the following sections some qualitative properties of the proposed numerical scheme defined by (4.27) are studied.

\subsubsection{Properties of intensity function and solution}

Lemma 4.1.1. The intensity functions defined by (4.4)-(4.7) are non-decreasing and satisfy

$$
\max _{\alpha} f(\alpha)=f\left(\alpha_{\max }\right)
$$

where $\alpha$ is difference between payoff and current option price. 
Proof. The intensity function $f_{1}$ is piecewise constant non decreasing function. Exponential functions $f_{2}$ and $f_{3}$ are increasing for positive parameter $\lambda$. The first derivative of $f_{4}$ is as follows

$$
f_{4}^{\prime}(\alpha)=\frac{2 \lambda^{3}}{\pi\left(1+\left(\lambda^{2} \alpha\right)^{2}\right)}>0
$$

and function $f_{4}$ is increasing.

For $x \geq 0$, the payoff is zero and $\alpha<0$. For $x<0$, we can obtain positive values of $\alpha$.

Now we prove that values at the left boundary do not exceed 1 for any time level and every intensity function. Let us introduce boundary function

$$
b(u)=(1-r k) u+k f(E(1-u))(1-u), \quad 0<u<1 .
$$

Firstly, let us consider function $f_{1}$. For $u \in(0,1)$ argument of function $f$ is positive and $f(E(1-u))=\lambda$. Thus,

$$
b^{\prime}(u)=1-(r+\lambda) k=0, \quad \text { if } k=\frac{1}{r+\lambda} .
$$

Therefore, function $b$ is monotone on $(0,1)$ for rest $k$ and its values are bounded

$$
0 \leq b(u) \leq \max \{k \lambda, 1-r k\}
$$

In order to guarantee that $b(u) \leq 1$ the following condition is found

$$
k \leq k_{1}=\frac{1}{r+\lambda} .
$$

For function $f_{2}$ the analogous procedure gives the condition $k \leq k_{2}$, where $k_{2}$ satisfies the equation

$$
k_{2}\left(r+\lambda e^{\lambda^{2} r k_{2}}\right)=1 .
$$

By the same procedure for $f_{3}$ one can find that $b(u)$ is increasing function for

$$
k \leq k_{3}=\frac{1}{r+2 \lambda},
$$

For $f_{4}$ this conditions takes the following form

$$
k \leq k_{4}=\frac{1}{r+\lambda+\frac{2}{\pi E \lambda}} .
$$




\subsection{Pricing of American option with rationality parameter}

Thus, maximum of $b(u)$ is reached at the point $u=1$ :

$$
b(1)=1-r k \leq 1
$$

Summarizing previous comments the following result has been established.

Lemma 4.1.2. With the previous notation, for each intensity function $f_{i}, i=$ $1,2,3,4$ defined by (4.4)-(4.7) correspondingly, there exists constant $k_{i}>0$ given by (4.28)-(4.31) correspondingly, such that at boundary point $x=0$ the approximation provided by formula (4.17) does not exceed one for $k<k_{i}$, that is:

$$
u_{0}^{n} \leq 1, \quad \forall n \leq N
$$

The following lemma establishes a bound of the difference between the solution at any time level and the transformed payoff function for a fixed mesh point $x_{j}, 0 \leq$ $j \leq M$, that will be useful for the study of qualitative properties of the proposed method.

Lemma 4.1.3. With the previous notation, the following estimation holds:

$$
\max _{j, n}\left(u_{j}^{0}-u_{j}^{n}\right) \leq \frac{r}{r+\lambda}\left(1-e^{-(r+\lambda) T}\right) .
$$

Proof. We consider the region $x<0$, because only in this region the positive argument of intensity function appears.

One can see that $u_{0}^{n}-u_{j}^{n} \leq 1-u_{j}^{0}$ for any fixed $n$ and for any $j$. Therefore for any fixed $n$ the following equality takes place

$$
\max _{j}\left(u_{j}^{0}-u_{j}^{n}\right)=u_{0}^{0}-u_{0}^{n}
$$

Thus, the boundary condition (4.17) is used. Firstly, $f_{1}$ is considered. The boundary condition (4.14) can be solved as ODE and its solution has the following form:

$$
u(\tau)=\frac{\lambda+r \exp (-(r+\lambda) \tau)}{r+\lambda},
$$

therefore for any fixed $n$

$$
u_{0}^{0}-u_{0}^{n}=\frac{r}{r+\lambda}\left(1-e^{-(r+\lambda) n k}\right) \leq \frac{r}{r+\lambda}\left(1-e^{-(r+\lambda) T}\right) .
$$


Now let us check this estimation for other intensity functions. From the definition of $f_{2}$ and boundary condition (4.17) one gets

$$
u_{0}^{n+1}=(1-r k) u_{0}^{n}+k \lambda e^{\lambda^{2} E\left(1-u_{0}^{n}\right)}\left(1-u_{0}^{n}\right) \leq(1-r k) u_{0}^{n}+k \lambda\left(1-u_{0}^{n}\right),
$$

if $u_{0}^{n} \leq 1$, that is logical condition that the option price could not exceed strike price. Thus, if we denote the corresponding approximation associated to $f_{1}$ by $\tilde{u}_{0}^{n}$, then one gets

$$
u_{0}^{0}-u_{0}^{n} \leq u_{0}^{0}-\tilde{u}_{0}^{n} \leq \frac{r}{r+\lambda}\left(1-e^{-(r+\lambda) T}\right) .
$$

Analogous technique is used for $f_{3}$ and $f_{4}$, since both of them take values greater than $\lambda$ for positive arguments. Therefore, for any considered intensity function the estimation 4.32 holds true.

Then, from the previous lemma we have

$$
\max _{\alpha} f(\alpha) \leq f\left(\frac{r}{r+\lambda}\left(1-e^{-(r+\lambda) T}\right)\right)
$$

Since we are interested in the value of intensity function for large values of the parameter $\lambda$, the following lemma states an asymptotic equivalence between $\lambda$ and the intensity function $f_{i}$ when $\lambda$ tends to infinity.

Lemma 4.1.4. From the specification of intensity functions, for any positive argument $\alpha$ and for $\lambda \rightarrow \infty$

$$
f_{i}(\alpha) \sim \lambda, \quad i=1,3,4
$$

More precisely,

$$
\lim _{\lambda \rightarrow \infty} \frac{f_{1}(\alpha)}{\lambda}=1, \quad \lim _{\lambda \rightarrow \infty} \frac{f_{3}(\alpha)}{\lambda}=\lim _{\lambda \rightarrow \infty} \frac{f_{4}(\alpha)}{\lambda}=2 .
$$

Proof. For positive argument $\alpha>0$

$$
\lim _{\lambda \rightarrow \infty} \frac{f_{1}(\alpha)}{\lambda}=\lim _{\lambda \rightarrow \infty} \frac{\lambda}{\lambda}=1 .
$$

For the third function:

$$
\lim _{\lambda \rightarrow \infty} \frac{f_{3}(\alpha)}{\lambda}=\lim _{\lambda \rightarrow \infty} \frac{2}{1+e^{-\lambda^{2} x}}=2 .
$$


For the fourth function:

$$
\lim _{\lambda \rightarrow \infty} \frac{f_{4}(\alpha)}{\lambda}=\lim _{\lambda \rightarrow \infty}\left(1+\frac{2}{\pi} \arctan \lambda^{2} x\right)=2\left(1+\frac{2}{\pi} \frac{\pi}{2}\right)=2 .
$$

Remark. Note that for the second intensity function:

$$
\lim _{\lambda \rightarrow \infty} \frac{f_{2}(\alpha)}{\lambda}=\lim _{\lambda \rightarrow \infty} e^{\lambda^{2} x}=\infty .
$$

After all these properties of the intensity functions, the following theorem can be established.

Theorem 4.1.1. There exists positive constant $C$ such that the rationality term of intensity functions $f_{1}, f_{3}$ and $f_{4}$ in equation (4.19) is bounded by $r C$ :

$$
f(\alpha) \alpha \leq r C .
$$

Proof. If argument $\alpha<0$, the intensity function tend to zero and product $f(\alpha) \alpha$ is negative. The product is zero for $\alpha=0$. For $\alpha>0$, from Lemma 4.1.1 the following estimation holds:

$$
f(\alpha) \alpha \leq f\left(\alpha_{\max }\right) \alpha_{\max }
$$

where, from Lemma 4.1.3, we have

$$
\alpha_{\max }=\max _{j, n}\left(u_{j}^{0}-u_{j}^{n}\right)=\frac{r}{r+\lambda}\left(1-e^{-(r+\lambda) T}\right) .
$$

Note that

$$
\lim _{\lambda \rightarrow \infty}\left(1-e^{-(r+\lambda) T}\right)=1 .
$$

Thus, using result of Lemma 4.1.4, we obtain

$$
\lim _{\lambda \rightarrow \infty} f\left(\alpha_{\max }\right) \alpha_{\max }=\lim _{\lambda \rightarrow \infty} c \frac{\lambda r}{r+\lambda}=r c,
$$

where $c=1$ for $f_{1}$, and $c=2$ for $f_{3}$ and $f_{4}$.

Therefore, there exists a constant $C \geq c$, such that

$$
f(\alpha) \alpha \leq r C .
$$


Note that under conditions

$$
h<\frac{\sigma^{2}}{\left|r-q-\frac{\sigma^{2}}{2}\right|}, \quad k<\frac{h^{2}}{\sigma^{2}+r h^{2}},
$$

the coefficients $b_{1}, b_{2}$ and $b_{3}$ defined in (4.26) are positive.

Furthermore, the term $k f_{j}^{n}$ in 4.27 is non-negative from its definition. Therefore, since the initial condition is non-negative, the values calculated by (4.27) are also non-negative.

In order to prove the monotonicity of the numerical solution, the mathematical induction principle can be used. At the initial time level, from (4.12) the decreasing behaviour of the solution is clear. Now, let us assume that at the $n$-th time level the monotonicity holds, i.e.

$$
u_{j}^{n} \geq u_{j+1}^{n}, \quad 0 \leq j<N_{x} .
$$

Next, let us prove that it holds true for $n+1$ under the explicit scheme:

$$
u_{j}^{n+1}-u_{j+1}^{n+1}=b_{1}\left(u_{j-1}^{n}-u_{j}^{n}\right)+b_{2}\left(u_{j}^{n}-u_{j+1}^{n}\right)+b_{3}\left(u_{j+1}^{n}-u_{j+2}^{n}\right)+k\left(f_{j}^{n}-f_{j+1}^{n}\right) .
$$

Since the coefficients $b_{1}, b_{2}$ and $b_{3}$ are non-negative under conditions 4.33 , by using hypothesis of induction we prove that the first three terms of the right hand side in 4.34) are non-negative.

\subsubsection{Stability and consistency}

Let us study stability of the scheme (4.27). For this purpose, we first choose the minimum index $m$, such that $u_{m}^{n+1}=\left\|u^{n+1}\right\|$. Note that if $m=0$ or $m=N_{x}$, then the scheme is stable by the definition.

Since the boundary condition $\mu_{1}$ is also obtained from a discretization of a PDE, let us study it. From the economical considerations, the value $u_{0}^{n}$ could not exceed 1 (the option price could no be higher than payoff).

Suppose that index $1 \leq m \leq N_{x}-1$, then

$$
\left|u_{m}^{n+1}\right|=\left|b_{1} u_{m-1}^{n}+b_{2} u_{m}^{n}+b_{3} u_{m+1}^{n}+k f_{m}^{n}\right| .
$$

Since all coefficients are positive, then

$$
\left|b_{1} u_{m-1}^{n}+b_{2} u_{m}^{n}+b_{3} u_{m+1}^{n}\right| \leq(1-r k)\left\|u^{n}\right\| .
$$




\subsection{Pricing of American option with rationality parameter}

The connection between $(n+1)$-th and $n$-th level is obtained:

$$
\left\|u^{n+1}||=\left|u_{m}^{n+1}\right| \leq\right\| u^{n}\|+k\| f^{n} \| .
$$

Let us present the solution $u=y+v$, where $y$ is a solution of the problem without rationality term ( $f \equiv 0), v$ is a solution of the problem with homogeneous boundary conditions $\left(\mu_{1} \equiv 0\right)$. Then, using estimation (4.35) one gets

$$
\left\|y^{n+1}\right\| \leq \max \left\{\max _{0 \leq i \leq N}\left|\mu_{1}^{i}\right|,\left\|y^{n}\right\|\right\} \leq \ldots \leq \max \left\{\max _{0 \leq i \leq N}\left|\mu_{1}^{i}\right|,\left\|y^{0}\right\|\right\} .
$$

On the other hand, we have

$$
\left\|v^{n+1}\right\| \leq\left\|v^{n}\right\|+k\left\|f^{n}\right\| \leq \ldots \leq T \max _{0 \leq i \leq N}\left\|f^{i}\right\| .
$$

Thus, we obtain

$$
\left\|u^{n}\right\| \leq\left\|y^{n}\right\|+\left\|v^{n}\right\| \leq \max \left\{\max _{0 \leq i \leq N}\left|\mu_{1}^{i}\right|,\left\|u^{0}\right\|\right\}+T \max _{0 \leq i \leq N}\left\|f^{i}\right\|,
$$

Therefore, under conditions (4.33), the scheme 4.27) is stable.

Assuming that $u(x, \tau)$ is continuously differentiable four times with respect to $x$ and twice with respect to $\tau$ and following the procedure of consistency study, one finds that the truncation error behaves

$$
T_{j}^{n}(\tilde{u})=O\left(h^{2}\right)+O(k) .
$$

\subsubsection{Numerical examples}

In the first example we consider a case with $q=0$, so that the underlying asset does not pay a dividend yield. The rest of the parameters are taken from an example in [103] where the classical American put with rational exercise is numerically solved. This choice allows as certain comparisons of the limit problem when the rationality parameter tends to infinity with some results in the literature.

Example 4.1.1. We consider American option pricing problem with the following parameters

$$
r=0.05, \quad \sigma=0.2, \quad T=3, \quad E=100 .
$$




\section{BEHAVIOURAL MODELLING OF OPTION PRICING}

\begin{tabular}{|c|c|c|c|c|}
\hline$\lambda$ & 90 & 100 & 110 & 120 \\
\hline 0 & 10.2394 & 6.9940 & 4.70856 & 3.1369 \\
1 & 12.1122 & 7.9539 & 5.2086 & 3.4015 \\
10 & 13.1528 & 8.6018 & 5.6071 & 3.6447 \\
100 & 13.2905 & 8.6981 & 5.6734 & 3.6898 \\
1000 & 13.3047 & 8.7082 & 5.6806 & 3.6948 \\
10000 & 13.3064 & 8.7097 & 5.6819 & 3.6961 \\
\hline Rational case & 13.3075 & 8.7106 & 5.6825 & 3.6964 \\
\hline
\end{tabular}

Table 4.1: Convergence to the true value with increasing $\lambda$ for family 4.4 for fixed $h=10^{-2}, k=10^{-4}$.

Using the notation $\tau$ for the time to maturity, for the explicit, implicit and Crank-Nicolson methods the numerical solutions at $\tau=T$ with step sizes $h=10^{-2}$ and $k=10^{-4}$ are compared with explicit methods after transformation (TrExpl) presented in Figures 4.1 and 4.2, for the intensity functions (4.4) and (4.5), respectively. In both cases the rationality parameter is $\lambda=100$. Note that with this value of the parameter the qualitative behaviour seems very close to the classical American put with rational exercise.

By using the explicit scheme for the transformed equation, the dependence of the solution with respect to the rational parameter $\lambda$ with family (4.4) and (4.5) for different asset prices is illustrated in Table 4.1 and 4.2 respectively. Results of families (4.6) and (4.7) are close to results in Table 4.1. It can be explained by fact that these two families od intensity functions are smooth analogue of functions described by (4.4). One can see that choosing family (4.5) brings near to rational case for smaller rationality parameter. On other hand, in this case condition 4.29) does not allow to take $\lambda>100$ for fixed $h=10^{-2}$ and $k=10^{-4}$. Additionally, the convergence of the proposed treatment of irrational exercise case to the classical American put value confirms that $\lambda$ fulfils the requirement of the definition of rational parameter.

Additionally, in Figure 4.3 for family (4.5) and different values $\lambda$ the American put option price is shown. Note that the case $\lambda=0$ corresponds to the European option, while $\lambda=1$ corresponds to a near zero rationality parameter which can be understood as a case with large irrational exercise and the value of the American put option is below the exercise value for small values of the asset. This situation 


\begin{tabular}{|c|c|c|c|c|}
\hline$\lambda$ & 90 & 100 & 110 & 120 \\
\hline 0 & 10.2394 & 6.9940 & 4.70856 & 3.1369 \\
1 & 12.5589 & 8.0809 & 5.1107 & 3.1409 \\
10 & 13.2911 & 8.6972 & 5.6711 & 3.6861 \\
100 & 13.3063 & 8.7093 & 5.6813 & 3.6953 \\
\hline Rational case & 13.3075 & 8.7106 & 5.6825 & 3.6964 \\
\hline
\end{tabular}

Table 4.2: Convergence to the true value with increasing $\lambda$ for family 4.5 for fixed $h=10^{-2}, k=10^{-4}$.

maybe caused by additional circumstances that prevent the owner to exercise, although the option price is bellow exercise prices. For $\lambda=100$ the large value of the rational parameter makes the solution to be like in the rational exercise case.

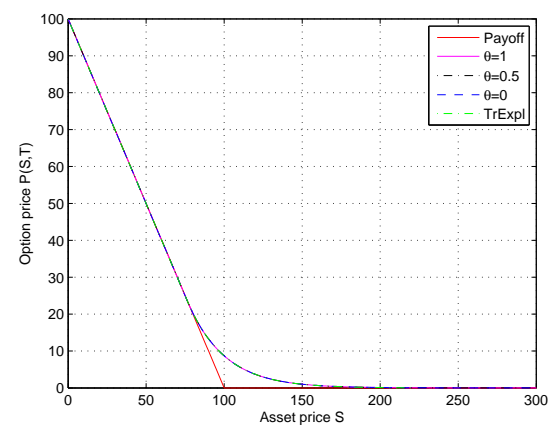

Figure 4.1: Numerical solution the intensity function belonging to family (4.4) with $\lambda=100$ for various values of $\theta$.

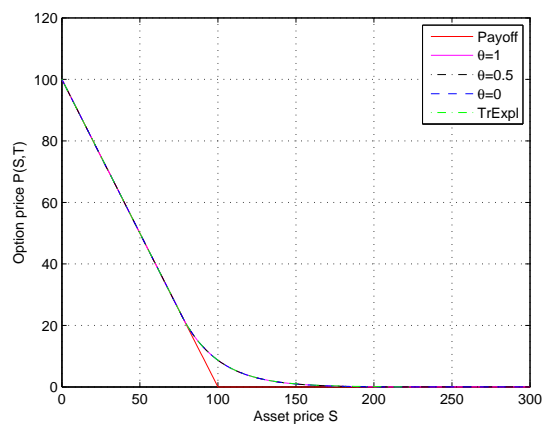

Figure 4.2: Numerical solution the intensity function belonging to family (4.5) with $\lambda=100$ for various values of $\theta$.

As indicated in [50], the family of intensity functions (4.5) allows that the owner of the American option may exercise too early or too late. Moreover, in the proof of the main theorem they develop a probabilistic analysis of the irrational exercise strategies. For this purpose, they classify how profitable an exercise strategy results 


\section{BEHAVIOURAL MODELLING OF OPTION PRICING}

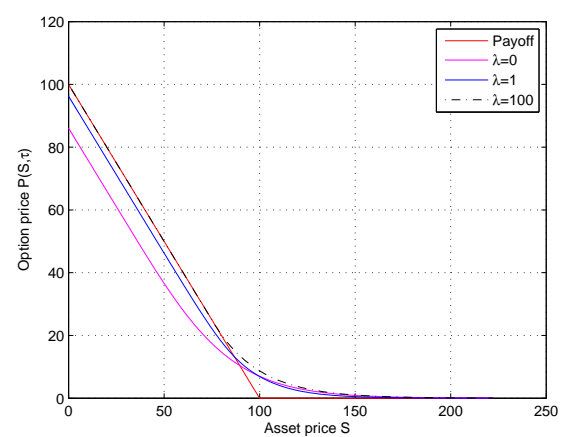

Figure 4.3: Numerical solution for the intensity function belonging to family 4.5 ) for various values of $\lambda$.

upon exercise. Thus, for a given tolerance $\epsilon$, they introduce the following sets:

$$
\begin{aligned}
\{\tau \operatorname{good}\} & =\left\{\left(1-x_{\tau}\right)^{+}-u\left(x_{\tau}, \tau\right)>0\right\} \\
\{\tau \text { ok }\} & =\left\{\left(1-x_{\tau}\right)^{+}-u\left(x_{\tau}, \tau\right) \in(-\epsilon, 0]\right\} \\
\{\tau \text { bad }\} & =\left\{\left(1-x_{\tau}\right)^{+}-u\left(x_{\tau}, \tau\right) \leq-\epsilon\right\}
\end{aligned}
$$

Note that if the exercise time was good, the holder of the American option looses some value due to irrational exercise. If the exercise time was ok, the owner would win at most the value $\epsilon$. Finally, if the exercise time was bad, then the holder gains more than $\epsilon$. Moreover, for a given threshold $\epsilon$ the intensity of exercising at bad times can be make arbitrarily uniformly small by decreasing the rationality parameter and the gain from regretting the exercises when $\tau$ is bad can be made arbitrarily small. In relation with this issue, in Figures 4.4 and 4.5 the option price with rationality parameters $\lambda=1$ and $\lambda=1000$, respectively, are presented. Furthermore, a colour code has been introduced to identify the good (green), ok (yellow) and bad (red) exercise strategies for a given tolerance $\epsilon=10^{-3}$.

At this point it seems noteworthy to illustrate an additional use of the solution of American pricing problems with irrational exercise. For this purpose, we first recall that the definition of rational parameter requires that when $\lambda$ tends to infinity, the irrational case tend to the rational one, which corresponds to the classical American option pricing problem. Therefore, for large values of $\lambda$ the numerical solutions of the problems with the previously proposed intensity functions can be used as alternatives to approximate the solution of the classical American option problem. In fact, for the case of intensity function $f_{1}$ we obtain the classical penalization technique to approximate the free boundary problem with unilateral constraint. 


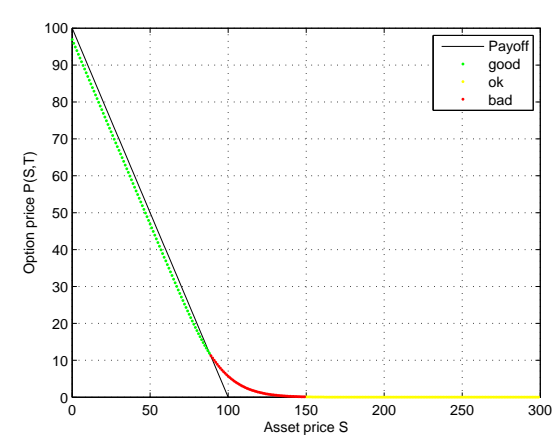

Figure 4.4: Option price with $\lambda=1$.

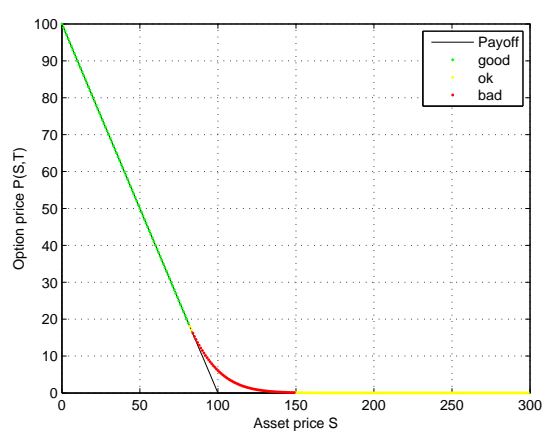

Figure 4.5: Option price with $\lambda=$ 1000 .

In the literature there exist many numerical methods for the American option pricing problem. In Table 4.3 a comparison of some different techniques in the literature is presented. In accordance with [103], the method of Leisen and Reimer [81] with 15001 steps is used as a "True value". We denote by $B \& S$ the Brennan and Schwartz algorithm [16]; Penalty is the penalty method described by Forsyth and Vetzal in [48]; operator splitting method is denoted by $O S$; the front-fixing explicit method proposed by Company et. al. in [24] is denoted by FF; $\mathrm{Han} \mathrm{Wu}$ is the method that uses accurate artificial boundary conditions described in [54]; $O C A$ is an abbreviation for optimal compact algorithm proposed by Tangman et. al. in [103]. The number of time steps for all methods is chosen to $N=600$ and $M=600$ is the number of spatial steps.

The analogous approximated results using the intensity functions approximations are shown in Table 4.4 with rationality parameter $\lambda=10^{4}$. In all cases an explicit finite difference method with transformation has been used with the same number of space steps $M$ and $N$ is chosen to guarantee the stability of the method and $M \times N=3.6 \cdot 10^{5}$. Note that these approximations are in good agreement with the limit problem corresponding to the rational case ("True" column in both tables) and also they result competitive with the ones provided by the alternative techniques for American options illustrated in Table 4.3 .

Example 4.1.2. In the present example we illustrate the different convergence rates of the proposed methods. Let us consider the problem (4.11) with parameters:

$$
r=0.05, \quad \sigma=0.2, \quad q=0.02, \quad T=1, \quad E=100 .
$$




\begin{tabular}{|c|c|c|c|c|c|c|c|}
\hline$S$ & True & B\&S & Penalty & OS & FF & Han Wu & OCA \\
\hline 80 & 20.2797 & 20.2793 & 20.2793 & 20.2795 & 20.2793 & 20.2803 & 20.2798 \\
90 & 13.3075 & 13.3072 & 13.3071 & 13.3074 & 13.3074 & 13.3075 & 13.3076 \\
100 & 8.7106 & 8.7102 & 8.7100 & 8.7104 & 8.7104 & 8.7103 & 8.7106 \\
110 & 5.6825 & 5.6822 & 5.6820 & 5.6824 & 5.6823 & 5.6823 & 5.6825 \\
120 & 3.6964 & 3.6961 & 3.6960 & 3.6963 & 3.6963 & 3.6965 & 3.6964 \\
\hline \multicolumn{2}{|c|}{ RMSE } & $3.435-4$ & $4.669-4$ & $1.4832-4$ & $2.297-4$ & $3.162-4$ & $6.325-5$ \\
CPU-time, sec. & 10.05 & 25.67 & 11.91 & 4.62 & 4.72 & 2.20 \\
\hline
\end{tabular}

Table 4.3: Comparison of different methods for the American option with rational exercise (classical problem).

\begin{tabular}{|c|c|c|c|c|c|}
\hline$S$ & True & $f_{1}(\alpha)$ & $f_{2}(\alpha)$ & $f_{3}(\alpha)$ & $f_{4}(\alpha)$ \\
\hline 80 & 20.2797 & 20.2799 & 20.2799 & 20.2802 & 20.2801 \\
\hline 90 & 13.3075 & 13.3073 & 13.3072 & 13.3074 & 13.3073 \\
\hline 100 & 8.7106 & 8.7102 & 8.7101 & 8.7103 & 8.7102 \\
\hline 110 & 5.6825 & 5.6822 & 5.6821 & 5.6823 & 5.6821 \\
\hline 120 & 3.6964 & 3.6963 & 3.6962 & 3.6964 & 3.6962 \\
\hline \multicolumn{2}{|c|}{ RMSE } & $2.683-4$ & $3.406-4$ & 2.793-4 & $3.347-4$ \\
\hline \multicolumn{2}{|c|}{ CPU-time, sec. } & 0.86 & 0.86 & 1.15 & 1.01 \\
\hline
\end{tabular}

Table 4.4: Comparison of the approximations with large rationality parameter $(\lambda=$ $\left.10^{4}\right)$ and a rational case reference approximation. 


\begin{tabular}{|c|cc|cc|}
\hline & \multicolumn{2}{|c|}{$\lambda=10^{4}$} & \multicolumn{2}{c|}{$\lambda=1$} \\
& $\gamma_{h}$ & $\gamma_{k}$ & $\gamma_{h}$ & $\gamma_{k}$ \\
\hline Explicit & 1.9944 & 1.0004 & 1.9567 & 1.0001 \\
Implicit & 1.9949 & 0.9997 & 1.9537 & 0.9992 \\
Crank-Nicolson & 1.9947 & 1.8465 & 1.9548 & 1.6307 \\
\hline Transform+Explicit & 2.0235 & 1.0008 & 2.0044 & 1.0001 \\
\hline
\end{tabular}

Table 4.5: Spatial and temporal convergence rates of explicit, implicit and CrankNicolson schemes for $\lambda=10^{4}$ and $\lambda=1$.

Since the exact solution of the problem is not analytically available, formula (1.22) is used for the estimation of the convergence rate in space, $\gamma_{h}$. We have provided a series of numerical results with fixed $k=2 \cdot 10^{-5}$ and $h=2 \cdot 10^{-2}$, $h / 2=10^{-2}$ and $h / 4=5 \cdot 10^{-3}$. The convergence rate $\gamma_{h}$ has been calculated by formula 1.22 for explicit, implicit and Crank-Nicolson schemes. The results are collected in Table 4.5, thus showing the expected orders for the approximation of the rational case $\left(\lambda=10^{4}\right)$ and the irrational exercise case $(\lambda=1)$.

Analogous to (1.22) formula is used in order to estimate the convergence rate in time, $\gamma_{k}$, for a fixed space step $h$. The space step is $h=5 \cdot 10^{-3}$, time steps are $k=2 \cdot 10^{-5}, k / 2=10^{-5}, k / 4=5 \cdot 10^{-6}$. The convergence rates $\gamma_{k}$ of explicit, implicit and Crank-Nicolson methods are presented in Table 4.5 .

As expected, the explicit and implicit methods are of order $O\left(h^{2}, k\right)$ while Crank-Nicolson method is of order $O\left(h^{2}, k^{2}\right)$.

Another important characteristic of the algorithms concerns to the required computational time. We compare the explicit method for the transformed equation with the different $\theta$-methods for the problem in the original variables. The space step is fixed to $h=10^{-2}$ and the time step is variable. The results are presented in Table 4.6, which shows as expected that explicit methods are conditionally stable and for $k=h=10^{-2}$ result to be unstable. Note that although for $k=10^{-} 3$ the explicit method for the problem in original variables is still unstable, after the transformation the stability condition for explicit scheme becomes weaker and the so called "Transform+Explicit" method exhibits an stable behaviour and involves almost the same computational time as the implicit methods for $k=10^{-2}$. Thus, the proposed transformation allows to construct a faster algorithm with a weaker stability condition. 


\begin{tabular}{|c|c|c|c|c|}
\hline$k$ & $10^{-2}$ & $10^{-3}$ & $10^{-4}$ & $10^{-5}$ \\
\hline Explicit & - & - & 1.8720 & 19.4221 \\
Crank-Nicolson & 0.0624 & 0.3120 & 2.8080 & 27.7058 \\
Implicit & 0.0312 & 0.2496 & 2.6364 & 26.8009 \\
\hline Transform+Explicit & - & 0.0780 & 0.7176 & 6.4584 \\
\hline
\end{tabular}

Table 4.6: CPU-time in seconds of proposed methods for fixed $h=10^{-} 2$ and various $k$.

Example 4.1.3. A problem with a dominant convection term is considered. For this purpose, the parameters are chosen as follows

$$
r=0.10, \quad \sigma=0.1, \quad q=0, \quad T=1, \quad E=100 .
$$

In this case, coefficient $b_{1}$ is negative. Moreover, for the intensity function 4.4 the rationality parameter $\lambda=100$ is chosen. In Figures 4.6 and 4.7 the numerical solution for $h=k=10^{-2}$ is shown on the left, while for $k=h^{2}=10^{-4}$ is presented on the right.

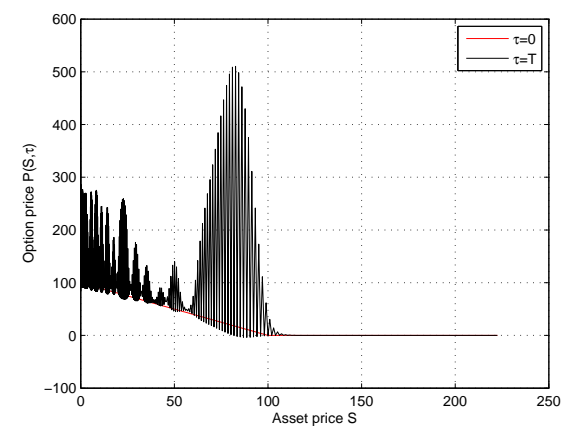

Figure 4.6: Oscillations of the solution of the problem with parameters 4.36 and $h=10^{-2}, k=10^{-2}$.

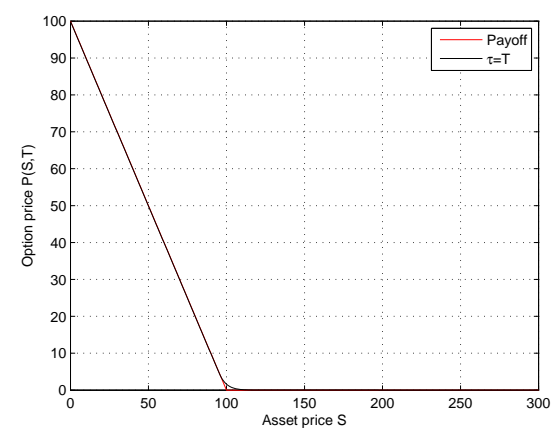

Figure 4.7: Stable numerical solution of the problem with parameters 4.36 with $h=10^{-2}, k=10^{-4}$.

The results of this section have been published in [25]. 


\subsection{Regime switching model with rationality parameter}

For an intensity function $f:[-E, E] \rightarrow[0, \infty)$ in the regime switching setting we assume that the relation between the profitability and the stochastic exercise intensity is $f\left((E-S)^{+}-V_{i}(S, \tau)\right)$ for each regime. After incorporating this term to the system of PDEs satisfied by the option price in describing the regime switching model (3.31)-3.35) one gets for $i=1, \ldots, I$ one obtains

$$
\begin{aligned}
\frac{\partial V_{i}}{\partial \tau}= & \frac{\sigma_{i}^{2}}{2} S^{2} \frac{\partial^{2} V_{i}}{\partial S^{2}}+r_{i} S \frac{\partial V_{i}}{\partial S}-r_{i} V_{i}+f\left((E-S)^{+}-V_{i}\right)\left((E-S)^{+}-V_{i}\right) \\
& +\sum_{l \neq i} q_{i, l}\left(V_{l}-V_{i}\right), \quad S>0,0<\tau \leq T
\end{aligned}
$$

for $i=1, \ldots, I$, jointly with the initial and boundary conditions:

$$
\begin{aligned}
V_{i}(S, 0)= & \max (E-S, 0), \\
\lim _{S \rightarrow \infty} V_{i}(S, \tau)= & 0, \\
\frac{\partial V_{i}}{\partial \tau}(0, \tau)= & -r_{i} V_{i}(0, \tau)+f\left(E-V_{i}(0, \tau)\right)\left(E-V_{i}(0, \tau)\right) \\
& +\sum_{l \neq i} q_{i, l}\left(V_{l}(0, \tau)-V_{i}(0, \tau)\right), \quad i=1, \ldots, I .
\end{aligned}
$$

Note that since the spatial domain is $S>0$ in rationality parameter model, an additional boundary condition at the point $S=0$ has to be included to treat the problem numerically. We assume that the PDE (4.37) holds at $S=0$, so that equation 4.40 is established.

Since a closed form solution is not available for the nonlinear system of equations (4.37)-(4.40), the solution has to be computed numerically. This section begins with a suitable substitution transforming the original PDE problem into a new one with constant coefficients in the differential part. After this a wide family of finite difference methods, known as weighted schemes [101], are developed. Relevant numerical analysis issues as positivity, stability and consistency are studied.

For the sake of clarity, let us recall some results in matrix analysis.

A matrix $B=\left(b_{i j}\right) \in \mathbb{R}^{m \times n}$ is a non-negative matrix, if $b_{i j} \geq 0$ for $1 \leq i \leq m$, $1 \leq j \leq n$. 
For any square matrix $B \in \mathbb{R}^{n \times n}$ the maximum of the moduli of its eigenvalues is called spectral radius $\rho(B)$. The $n \times n$ identity matrix is denoted by $I$.

A $n \times n$ matrix $B$ is called an M-matrix if it can be expressed in the form $B=s I-\tilde{B}$, where $\tilde{B}=\left(\tilde{b}_{i j}\right)$ with $\tilde{b}_{i j} \geq 0$, and $s \geq \rho(\tilde{B})$. A matrix $B$ is a nonsingular M-matrix if and only if it is inverse-positive, that is $B^{-1} \geq 0$ (see statement $F_{15}$ in [93]). Matrix $B$ having all positive diagonal elements is a M-matrix if there exists a positive diagonal matrix $D$, such that $B D$ is strictly diagonally dominant (see statement $N_{39}$ in [93]).

\subsubsection{Weighted finite difference scheme for PDE problem}

In order to construct an effective finite difference scheme with constant coefficients in the differential part, let us introduce the following normalized transformation

$$
x=\ln \frac{S}{E}, \quad u_{i}=\frac{V_{i}(S, \tau)}{E}, \quad i=1, \ldots, I .
$$

Then, problem 4.37)-(4.40) takes the following equivalent form:

$$
\begin{aligned}
\frac{\partial u_{i}}{\partial \tau} & =\frac{\sigma_{i}^{2}}{2} \frac{\partial^{2} u}{\partial x^{2}}+\left(r_{i}-\frac{\sigma_{i}^{2}}{2}\right) \frac{\partial u_{i}}{\partial x}-r_{i} u_{i}+\sum_{l \neq i} q_{i, l}\left(u_{l}-u_{i}\right) \\
& +f\left(E\left(1-e^{x}\right)^{+}-E u_{i}\right)\left(\left(1-e^{x}\right)^{+}-u_{i}\right), i=1, \ldots, I,
\end{aligned}
$$

with the new initial and boundary conditions

$$
\begin{aligned}
u_{i}(x, 0) & =\left(1-e^{x}\right)^{+}, \\
\lim _{x \rightarrow \infty} u_{i}(x, \tau) & =0, \\
\lim _{x \rightarrow-\infty} \frac{\partial u_{i}}{\partial \tau}(x, \tau) & =\lim _{x \rightarrow-\infty}-r_{i} u_{i}(x, \tau) \\
& +f\left(E\left(1-u_{i}(x, \tau)\right)\right)\left(1-u_{i}(0, \tau)\right) \\
& +\sum_{l \neq i} q_{i, l}\left(u_{l}(x, \tau)-u_{i}(x, \tau)\right) .
\end{aligned}
$$

The bounded computational domain is chosen as $\left[x_{\min }, x_{\max }\right] \times[0, T]$, where $x_{\min }=-3, x_{\max }=3$, that is sufficiently large to translate limit conditions 4.43 and (4.44) into boundary conditions at $x=x_{\min }$ and $x=x_{\text {max }}$, respectively. A uniform grid of $M+1$ spatial nodes and $N+1$ temporal nodes is introduced with 
step sizes $h=\frac{x_{\max }-x_{\min }}{M}$ and $k=\frac{T}{N}$, respectively. The nodes are denoted as follows

$$
x_{j}=x_{\text {min }}+j h, j=0, \ldots, M, \quad \tau^{n}=n k, n=0, \ldots, N .
$$

For each regime $i=1, \ldots, I$, the finite differences approximation of the solution at the node $\left(x_{j}, \tau^{n}\right)$ is denoted by $u_{i, j}^{n}$. Then, the weighted finite difference scheme with parameter $\theta \in[0,1]$, by using central differences in space and forward difference in time takes the following form

$$
\begin{array}{r}
-\theta a_{i} u_{i, j-1}^{n+1}+b_{i} u_{i, j}^{n+1}-\theta c_{i} u_{i, j+1}^{n+1}=(1-\theta) a_{i} u_{i, j-1}^{n}+\tilde{b}_{i} u_{i, j}^{n}+(1-\theta) c_{i} u_{i, j+1}^{n} \\
+k f_{i, j}^{n}\left(u_{i, j}^{0}-u_{i, j}^{n}\right)+k \sum_{l \neq i} q_{i, l}\left(u_{l, j}^{n}-u_{i, j}^{n}\right), \quad j=1, \ldots, M-1, n=0, \ldots, N-1,
\end{array}
$$

where the involved coefficients are

$$
\begin{aligned}
a_{i} & =\frac{\sigma_{i}^{2}}{2} \frac{k}{h^{2}}-\left(r_{i}-\frac{\sigma_{i}^{2}}{2}\right) \frac{k}{2 h}, \\
b_{i} & =1+\theta\left(\sigma_{i}^{2} \frac{k}{h^{2}}+r_{i} k\right), \\
\tilde{b}_{i} & =1-(1-\theta)\left(\sigma_{i}^{2} \frac{k}{h^{2}}+r_{i} k\right), \\
c_{i} & =\frac{\sigma_{i}^{2}}{2} \frac{k}{h^{2}}+\left(r_{i}-\frac{\sigma_{i}^{2}}{2}\right) \frac{k}{2 h},
\end{aligned}
$$

and the rationality function term is denoted by $f_{i, j}^{n}=f\left(E\left(u_{i, j}^{0}-u_{i, j}^{n}\right)\right)$. Note that the $\theta$-method is used for the differential part while the rest terms are treated explicitly for the computational convenience [71]. Note that case $\theta=0, \theta=1 / 2$ and $\theta=1$ corresponds to the so called fully explicit scheme, Crank-Nicolson and fully implicit schemes, respectively.

Initial condition is discretized as follows

$$
u_{i, j}^{0}=\left(1-e^{x_{j}}\right)^{+}, \quad j=0, \ldots, M, i=1, \ldots, I
$$

For each regime $i=1, \ldots, I$ and each time level $n=0, \ldots, N-1$, the discrete form of the boundary condition at the point $x_{0}=x_{\text {min }}$ for each regime $i=1, \ldots, I$ 
and any time level $n=0, \ldots, N-1$ is obtained by using a forward in time explicit finite difference scheme, thus leading to

$$
u_{i, 0}^{n+1}=\left(1-r_{i} k\right) u_{i, 0}^{n}+k f_{i, 0}^{n}\left(1-u_{i, 0}^{n}\right)+k \sum_{l \neq i} q_{i, l}\left(u_{l, 0}^{n}-u_{i, 0}^{n}\right) .
$$

Also for each regime $i=1, \ldots, I$ and each time level $n=0, \ldots, N-1$, at the boundary point $x_{M}=x_{\max }$ the zero boundary condition $u_{i, M}^{n+1}=0$ is imposed.

Since the nonlinear terms in 4.45 are taken from the previous time level, the system of equations (4.45) is linear with tridiagonal matrix, so that it can be solved by Thomas algorithm.

\subsubsection{Qualitative properties of the scheme}

In this section, some qualitative properties of the proposed numerical method (positivity, stability and consistency) are studied. First, we start with the approximation at $x=x_{\min }=x_{0}$. Since the value at this left boundary is described by a differential equation, one has to guarantee that solution is stable and oscillations do not occur at that point. The following Lemma provides conditions for boundedness of the numerical solution at the point $x_{\text {min }}$.

Lemma 4.2.1. With the previous notation, if

$$
k<\min _{1 \leq i \leq I} \frac{1}{r_{i}+C \lambda-q_{i, i}}
$$

then we have

$$
0 \leq u_{i, 0}^{n} \leq 1, \quad i=1, \ldots, I, n=0, \ldots, N,
$$

where the constant $C$ appearing in 4.48 is defined as

$$
C=\lim _{\lambda \rightarrow \infty} \frac{f^{\lambda}(x)}{\lambda}
$$

so that $C=1$ for $f_{1}$, and $C=2$ for $f_{2}$ and $f_{3}$.

Proof. Let us consider boundary condition 4.47). Note that $u_{i, 0}^{0} \in[0,1]$. Next, assume that $u_{i, 0}^{n} \in[0,1]$ for each regime $i=1, \ldots, I$ and fixed $n$. Then 


$$
\begin{aligned}
u_{i, 0}^{n+1} & \leq\left(1-k r_{i}\right) u_{i, 0}^{n}+k f_{i, 0}^{n}\left(1-u_{i, 0}^{n}\right)-k q_{i, i}\left(1-u_{i, 0}^{n}\right) \\
& \leq\left(1-k r_{i}-k f_{i, 0}^{n}+k q_{i, i}\right) u_{i, 0}^{n}+k\left(f_{i, 0}^{n}-q_{i, i}\right) \leq 1
\end{aligned}
$$

provided that

$$
1-k\left(r_{i}+f_{i, 0}^{n}-q_{i, i}\right) \geq 0 .
$$

In that case, since $u_{i, 0}^{n} \leq 1,4.50$ holds true if

$$
1-k r_{i}-k f_{i, 0}^{n}+k q_{i, i} \leq 1-k\left(f_{i, 0}^{n}-q_{i, i}\right),
$$

that is obvious for any $k$, such that 4.51 holds. Therefore, for 4.49 ) it is necessary to choose $k$ satisfying

$$
k \leq \frac{1}{r_{i}+f_{i, 0}^{n}-q_{i, i}} .
$$

Since 4.52 has to be fulfilled for any fixed $n, f_{i, 0}^{n}$ can be bounded by the limit values: $\lambda$ for $f_{1}$ and $2 \lambda$ for $f_{2}$ and $f_{3}$. Therefore, condition 4.48) is proved. Note, that the non-negativity of $u_{i, 0}^{n}$ follows from 4.47). Once rewritten in the following form

$$
u_{i, 0}^{n+1}=\left(1-k\left(r_{i}-q_{i, i}\right)\right) u_{i, 0}^{n}+k f_{i, 0}^{n}\left(1-u_{i, 0}^{n}\right)+k \sum_{l \neq i} q_{i, l} u_{l, 0}^{n},
$$

since each term in 4.53 is non-negative.

All the forthcoming results are valid under condition (4.48). Next result guarantees the positivity of the numerical solution $\left\{u_{i, j}^{n}\right\}$ under certain conditions on the step sizes.

Theorem 4.2.1. With the previous notation, the finite difference scheme (4.45) preserves the non-negativity of the numerical solution under the following conditions

$$
h<\min _{i=1, \ldots, I} \frac{\sigma_{i}^{2}}{\left|r_{i}-\frac{\sigma_{i}^{2}}{2}\right|}, \quad k<\min _{i=1, \ldots, I} \frac{h^{2}}{\sigma_{i}^{2}+\left(r_{i}-q_{i, i}+2 \lambda\right) h^{2}} .
$$

In the case that $r_{i}=\frac{\sigma_{i}^{2}}{2}$, only the second inequality of 4.54 is needed for all $h>0$. 
Proof. First, let us consider $\theta=0$. In this case, the scheme (4.45) can be rewritten in following form

$$
u_{i, j}^{n+1}=a_{i} u_{i, j-1}^{n}+\left(\tilde{b}_{i}+q_{i, i} k-f_{i, j}^{n} k\right) u_{i, j}^{n}+c_{i} u_{i, j+1}^{n}+k f_{i, j}^{n} u_{i, j}^{0}+k \sum_{l \neq i} q_{i, l} u_{l, j}^{n},
$$

where $a_{i}, \tilde{b}_{i}$ and $c_{i}$ are defined by (4.46). Under conditions 4.54 the coefficients $a_{i},\left(\tilde{b}_{i}+q_{i, i} k-f_{i, j}^{n} k\right)$ and $c_{i}$ in 4.55 are positive. Moreover, note that the value of the intensity function $f_{i, j}^{n}$ is non-negative by the definition and the last term is a linear combination of non-negative elements from the previous time level. Thus, providing positive solution $\left\{u_{i, j}^{n}\right\}$ at the time level $n$, non-negativity of $\left\{u_{i, j}^{n+1}\right\}$ is established.

For the remaining values of $\theta$, let us consider the vector form of scheme 4.45

$$
A_{i} u_{i}^{n+1}=\beta_{i}^{n},
$$

where $u_{i}^{n+1}=\left[\begin{array}{llll}u_{i, 1}^{n+1} & u_{i, 2}^{n+1} & \ldots & u_{i, M-1}^{n+1}\end{array}\right]^{T}, A_{i}$ is the tridiagonal constant matrix

$$
A_{i}=\left(\begin{array}{rrrrrr}
b_{i} & -\theta c_{i} & 0 & 0 & \ldots & 0 \\
-\theta a_{i} & b_{i} & -\theta c_{i} & 0 & \ldots & 0 \\
\ddots & \ddots & \ddots & \ddots & \ddots & \ddots \\
0 & 0 & \ldots & -\theta a_{i} & b_{i} & -\theta c_{i} \\
0 & 0 & \ldots & 0 & -\theta a_{i} & b_{i}
\end{array}\right)
$$

and $\beta_{i}^{n}$ is a vector of $M-1$ components $\beta_{i, j}^{n}$, such that

$$
\beta_{i, j}^{n}=(1-\theta) a_{i} u_{i, j-1}^{n}+\tilde{b}_{i} u_{i, j}^{n}+(1-\theta) c_{i} u_{i, j+1}^{n}+k f_{i, j}^{n}\left(u_{i, j}^{0}-u_{i, j}^{n}\right)+k \sum_{l \neq i} q_{i, l}\left(u_{l, j}^{n}-u_{i, j}^{n}\right)
$$

for $j=2, \ldots, M-1$, and

$$
\beta_{i, 1}^{n}=(1-\theta) a_{i} u_{i, 0}^{n}+\tilde{b}_{i} u_{i, 1}^{n}+(1-\theta) c_{i} u_{i, 2}^{n}+k f_{i, 1}^{n}\left(u_{i, 1}^{0}-u_{i, 1}^{n}\right)+k \sum_{l \neq i} q_{i, l}\left(u_{l, 1}^{n}-u_{i, 1}^{n}\right)+\theta a_{i} u_{i, 0}^{n+1} .
$$

Note that from 4.46, if conditions 4.54) hold, then the coefficients $a_{i}$ and $c_{i}$ are non-negative and also we have 


$$
0 \leq \theta\left(a_{i}+c_{i}\right)<b_{i}
$$

Consequently $A_{i}$ is a strictly diagonally dominant, and then a non-singular Mmatrix. Therefore, the inverse matrix $A_{i}^{-1}$ does not contain negative elements [93]. As it has been shown for $\theta=0$, if conditions (4.54) are then $\beta_{i}^{n}$ is a non-negative vector. Therefore, the solution

$$
u_{i}^{n+1}=A_{i}^{-1} \beta_{i}^{n}
$$

is non-negative for all $\theta \in[0,1]$.

Further we prove that under condition (4.48), constraints (4.54) are sufficient for the stability of the explicit scheme, while the stability of scheme (4.45) for $\theta \geq \frac{1}{2}$ does not require extra conditions.

Stability analysis is provided following the von Neumann approach. It is usually applied to schemes for linear equations. However, such method has been used also for the variable coefficients case by freezing at each level (see [39], [53], [102], p. 59).

Theorem 4.2.2. With the previous notation, explicit finite difference scheme (4.45) with $\theta=0$ is conditionally stable with stability conditions (4.54).

Proof. As in previous section, let us denote the regime index by $R$. An initial error vector for every regime $g_{R}^{0}, R=1, \ldots, I$, is expressed as a finite complex Fourier series, so that at $x_{j}$ the solution $u_{i, j}^{n}$ can be rewritten as follows

$$
u_{R, j}^{n}=g_{R}^{n} e^{i j \phi}, \quad j=1, \ldots, M-1, R=1, \ldots, I,
$$

where $i=(-1)^{1 / 2}$ is the imaginary unit and $\phi$ is a phase angle. Then, the scheme is stable if for every regime $R=1, \ldots, I$ the amplification factor $G_{R}=\frac{g_{R}^{n+1}}{g_{R}^{n}}$ satisfies the relation

$$
\left|G_{R}\right| \leq 1+K k=1+O(k),
$$

where the positive number $K$ is independent on $h, k$ and $\phi$ (see [101], p. 68, [102], p. 50). 
For the sake of simplicity of the notation, the index of the regime $R$ is skipped in the unknowns, the coefficients and the parameters, understanding that the calculations are done for each regime. The numerical scheme 4.55 is rewritten in the following way:

$$
\begin{aligned}
g^{n+1} e^{i j \phi}= & a g^{n} e^{i(j-1) \phi}+\left(\tilde{b}_{i}+q_{i, i} k\right) g^{n} e^{i j \phi}+c g^{n} e^{i(j+1) \phi} \\
& +k f\left(E\left(g^{0}-g^{n}\right) e^{i j \phi}\right)\left(\left(g^{0}-g^{n}\right) e^{i j \phi}\right)+k \sum_{l \neq R} q_{R, l} g_{l}^{n} e^{i j \phi} .
\end{aligned}
$$

Next, dividing both parts by $g^{n} e^{i j \phi}$ and denoting

$$
\begin{aligned}
& A_{1}=a e^{-i \phi}+\tilde{b}_{i}+q_{i, i} k+c e^{i \phi}, \\
& A_{2}=f\left(E\left(g^{0}-g^{n}\right) e^{i j \phi}\right)\left(\frac{g^{0}}{g^{n}}-1\right), \\
& A_{3}=\sum_{l \neq R} q_{R, l} \frac{g_{l}^{n}}{g^{n}},
\end{aligned}
$$

then expression (4.62) takes the following form:

$$
G=A_{1}+k\left(A_{2}+A_{3}\right) .
$$

Next, if $\left|A_{1}\right| \leq 1,\left|A_{2}+A_{3}\right| \leq K$ then expression (4.65) satisfies (4.61). Thus,

$$
\left|A_{1}\right|^{2}=\left(1-2 \frac{\sigma^{2} k \sin ^{2} \frac{\phi}{2}}{h^{2}}-(r-q) k\right)^{2}+\frac{k^{2}}{h^{2}}\left(r-\frac{\sigma^{2}}{2}\right)^{2} \sin ^{2} \phi .
$$

Moreover, when positivity conditions (4.54) are fulfilled then

$$
\left\{\begin{array}{l}
k\left(\frac{\sigma^{2}}{h^{2}}+(r-q)\right) \leq 1, \\
k\left(r-\frac{\sigma^{2}}{2}+\sigma^{2}(r-q)\right) \leq \sigma^{2}
\end{array}\right.
$$

so that $\left|A_{1}\right| \leq 1$.

Now, the coupling term $A_{3}$ can be bounded as follows

$$
\left|A_{3}\right|=\sum_{l \neq R} q_{R, l}\left|\frac{g_{l}^{n}}{g^{n}}\right| \leq \max _{l \neq R}\left|\frac{g_{l}^{n}}{g^{n}}\right|\left|q_{R, R}\right|=\left|\frac{g_{l_{0}(n)}^{n}}{g^{n}}\right|\left|q_{R, R}\right|=C(n),
$$

where $C(n)$ is independent on $\phi, h$ and $k$ and depends only on the frozen index $n$.

Since intensity functions (4.4)-4.7) are bounded, one can conclude that $A_{2}$ is also bounded by some constant independent on $h, k$ and $\phi$. 
Analogous approach is used to study stability of the scheme (4.45) with $\theta>0$.

Theorem 4.2.3. With the previous notation the scheme $(4.45)$ is stable for $\theta \geq \frac{1}{2}$.

Proof. The procedure of von Neumann method is retaken (see [101], p. 68, [102], p.50) and the solution is presented in the form (4.60). Then, after dividing both sides of the identity by $g^{n} e^{i j \phi}$, the numerical scheme 4.45 takes the following form:

$$
\begin{aligned}
& \frac{g^{n+1}}{g^{n}}\left[1+k \theta\left(\frac{\sigma^{2}}{h^{2}}+r-\frac{\sigma^{2}}{2 h^{2}}\left(e^{-i \phi}+e^{i \phi}\right)+\frac{r-\frac{\sigma^{2}}{2}}{2 h}\left(e^{-i \phi}-e^{i \phi}\right)\right)\right] \\
& =1+k(1-\theta)\left[-\frac{\sigma^{2}}{h^{2}}-r+\frac{\sigma^{2}}{2 h^{2}}\left(e^{-i \phi}+e^{i \phi}\right)-\frac{r-\frac{\sigma^{2}}{2}}{2 h}\left(e^{-i \phi}-e^{i \phi}\right)\right] \\
& +k\left[f_{i, j}^{n}\left(\frac{g^{0}}{g^{n}}-1\right)+\sum_{l \neq R} q_{R, l}\left(\frac{g_{l}^{n}}{g^{n}}-1\right)\right] .
\end{aligned}
$$

Let us denote

$$
\begin{aligned}
& A_{1}=1+k \theta\left(\frac{\sigma^{2}}{h^{2}}+r-\frac{\sigma^{2}}{2 h^{2}}\left(e^{-i \phi}+e^{i \phi}\right)+\frac{r-\frac{\sigma^{2}}{2}}{2 h}\left(e^{-i \phi}-e^{i \phi}\right)\right), \\
& A_{2}=1-k(1-\theta)\left[\frac{\sigma^{2}}{h^{2}}+r-\frac{\sigma^{2}}{2 h^{2}}\left(e^{-i \phi}+e^{i \phi}\right)+\frac{r-\frac{\sigma^{2}}{2}}{2 h}\left(e^{-i \phi}-e^{i \phi}\right)(4 . .70)\right.
\end{aligned}
$$

Note that

$$
\begin{aligned}
\left|A_{1}\right|^{2} & =1+\theta^{2}\left(2 \sigma^{2} \frac{k}{h^{2}} \sin ^{2} \frac{\phi}{2}+r k\right)^{2}+2 \theta\left(2 \sigma^{2} \frac{k}{h^{2}} \sin ^{2} \frac{\phi}{2}+r k\right) \\
& +\left(\theta \frac{k}{h}\left(r-\frac{\sigma^{2}}{2}\right) \sin \phi\right)^{2}>1 .
\end{aligned}
$$

Next, taking into account that the rationality term is bounded and (4.67), expression 4.68) is bounded as follows

$$
\left|A_{1}\right||G| \leq\left|A_{2}\right|+C(n) k .
$$


Since last term in 4.68 is order $O(k)$ and $\left|A_{1}\right|>1$, due to the condition (4.61), for stability it is sufficient to guarantee that

$$
\frac{\left|A_{2}\right|}{\left|A_{1}\right|} \leq 1
$$

Next, note that inequality 4.72 is equivalent to

$$
\begin{array}{r}
(1-2 \theta)\left[4 \sigma^{2} \frac{k^{2}}{h^{2}} \sin ^{2} \frac{\phi}{2}\left(\frac{\sigma^{2}}{h^{2}} \sin ^{2} \frac{\phi}{2}+r\right)+r^{2} k^{2}+\left(r-\frac{\sigma^{2}}{2}\right)^{2} \frac{k^{2}}{h^{2}} \sin ^{2} \phi\right] \\
-4 \sigma^{2} \frac{k}{h^{2}} \sin ^{2} \frac{\phi}{2}-2 r k<0
\end{array}
$$

that holds true for any $\theta \geq \frac{1}{2}$. Therefore, for $\theta \geq \frac{1}{2}$ the scheme is stable in accordance with the known property of the weighted scheme for linear equations (see [101], p. 29).

Remark. For $0 \leq \theta<\frac{1}{2}$ conditions 4.54 are sufficient for stability.

Now let us study consistency of the finite difference scheme (4.45) with PDE (4.41). Following the definition given in [101], consistency of a numerical scheme with respect to a partial differential equation means that the exact solution of the PDE approximates well the exact theoretical solution of the finite difference scheme as the temporal and spatial discretization steps size tend to zero.

Let us rewrite the finite difference scheme 4.45 with parameter $\theta \in[0,1]$, for every fixed regime $i=1, \ldots, I, 1 \leq j \leq M-1,0 \leq n \leq N-1$, as follows

$$
\begin{aligned}
F\left(u_{i, j}^{n+\theta}\right) & =\frac{u_{i, j}^{n+1}-u_{i, j}^{n}}{k}-\theta \frac{\sigma_{i}^{2}}{2 h^{2}}\left(u_{i, j-1}^{n+1}-2 u_{i, j}^{n+1}+u_{i, j+1}^{n+1}\right) \\
& -(1-\theta) \frac{\sigma_{i}^{2}}{2 h^{2}}\left(u_{i, j-1}^{n}-2 u_{i, j}^{n}+u_{i, j+1}^{n}\right)-\left(r_{i}-\frac{\sigma_{i}^{2}}{2}\right) \frac{\theta}{2 h}\left(u_{i, j+1}^{n+1}-u_{i, j-1}^{n+1}\right) \\
& -\left(r_{i}-\frac{\sigma_{i}^{2}}{2}\right) \frac{1-\theta}{2 h}\left(u_{i, j+1}^{n+1}-u_{i, j-1}^{n+1}\right)+r_{i} \theta u_{i, j}^{n+1}+r_{i}(1-\theta) u_{i, j}^{n} \\
& -f_{i, j}^{n}\left(u_{i, j}^{0}-u_{i, j}^{n}\right)-\sum_{l \neq i} q_{i, l}\left(u_{l, j}^{n}-u_{l, j}^{n}\right)=0 .
\end{aligned}
$$

Furthermore, let us rewrite the PDE system 4.41 for every fixed $i=1, \ldots, I$ as follows 


$$
\begin{aligned}
L\left(u_{i}\right)= & \frac{\partial u_{i}}{\partial \tau}-\frac{\sigma_{i}^{2}}{2} \frac{\partial^{2} u_{i}}{\partial x^{2}}-\left(r_{i}-\frac{\sigma_{i}^{2}}{2}\right) \frac{\partial u_{i}}{\partial x}+\left(r_{i}-q_{i, i}\right) u_{i} \\
& -f\left(\left(1-e^{x}\right)^{+}-u_{i}\right)-\sum_{l \neq i} q_{i, l} u_{l}=0, \quad i=1, \ldots, I .
\end{aligned}
$$

Next, by denoting the value of the exact solution of the PDE at the mesh point $\left(x_{j}, \tau^{n}\right)$ by $\tilde{u}_{i, j}^{n}=u_{i}\left(x_{j}, \tau^{n}\right)$, the local truncation error $T_{i, j}^{n}$ is

$$
T_{i, j}^{n}\left(\tilde{u}_{i}\right)=F\left(\tilde{u}_{i, j}^{n}\right)-L\left(\tilde{u}_{i, j}^{n}\right) .
$$

Note that if $T_{i, j}^{n}$ tends to zero as $h \rightarrow 0$ and $k \rightarrow 0$, then the consistency of the scheme is guaranteed. Assuming that $u_{i}(x, \tau), i=1, \ldots, I$, is continuously differentiable four times with respect to ] $x$ and three times with respect to $\tau$ [101] and using the Taylor's expansion around the point $\left(x_{j}, \tau^{n+\theta}\right)$, one gets

$$
\begin{aligned}
u_{i}\left(x_{j}, \tau^{n}\right)= & u_{i}\left(x_{j}, \tau^{n+\theta}\right)-k \theta \frac{\partial u_{i}}{\partial \tau}\left(x_{j}, \tau^{n+\theta}\right) \\
& +\frac{k^{2} \theta^{2}}{2} \frac{\partial^{2} u_{i}}{\partial \tau^{2}}\left(x_{j}, \tau^{n+\theta}\right)+O\left(k^{3}\right), \\
u_{i}\left(x_{j}, \tau^{n+1}\right)= & u_{i}\left(x_{j}, \tau^{n+\theta}\right)+k(1-\theta) \frac{\partial u_{i}}{\partial \tau}\left(x_{j}, \tau^{n+\theta}\right) \\
& +\frac{k^{2}(1-\theta)^{2}}{2} \frac{\partial^{2} u_{i}}{\partial \tau^{2}}\left(x_{j}, \tau^{n+\theta}\right)+O\left(k^{3}\right) .
\end{aligned}
$$

Thus, we have

$$
\frac{\partial u_{i}}{\partial \tau}\left(x_{j}, \tau^{n+\theta}\right)=\frac{\tilde{u}_{i, j}^{n+1}-\tilde{u}_{i, j}^{n}}{k}+(1-2 \theta) k \frac{\partial^{2} u_{i}}{\partial \tau^{2}}\left(x_{j}, \tau^{n+\theta}\right)+O\left(k^{2}\right)
$$

Note that the choice $\theta=\frac{1}{2}$ removes the term $O(k)$, so that only in this case we get a second order in time approximation.

Analogously, one obtains that

$$
\begin{aligned}
\frac{\partial u_{i}}{\partial x}\left(x_{j}, \tau^{n+\theta}\right)= & \theta \frac{\tilde{u}_{j+1}^{n+1}-\tilde{u}_{j-1}^{n+1}}{2 h}+(1-\theta) \frac{\tilde{u}_{j+1}^{n}-\tilde{u}_{j-1}^{n}}{2 h}+O\left(h^{2}\right), \\
\frac{\partial^{2} u_{i}}{\partial x^{2}}\left(x_{j}, \tau^{n+\theta}\right)= & \theta \frac{\tilde{u}_{j+1}^{n+1}-2 \tilde{u}_{j}^{n+1}+\tilde{u}_{j-1}^{n+1}}{h^{2}} \\
& +(1-\theta) \frac{\tilde{u}_{j+1}^{n}-2 \tilde{u}_{j}^{n+1}+\tilde{u}_{j-1}^{n}}{h^{2}}+O\left(h^{2}\right) .
\end{aligned}
$$


Next, by replacing 4.76, 4.77) and 4.78) into 4.74, the consistency of the scheme (4.73) for the PDE system (4.74) is established and the truncation error (4.75) takes the following form

$$
T_{j}^{n}\left(\tilde{u}_{i}\right)=(1-2 \theta) k \frac{\partial^{2} u_{i}}{\partial \tau^{2}}\left(x_{j}, \tau^{n+\theta}\right)+O\left(k^{2}\right)+O\left(h^{2}\right) \quad \forall i=1, \ldots, I .
$$

From the previous equation it follows the order of convergence in $k$ and $h$ of the methods for $\theta=0,1 / 2,1$. These orders of convergence will be illustrated in the forthcoming section of numerical results.

\subsubsection{Numerical examples}

In the next example we show that for sufficiently large values of rationality parameter the solution of the problem (4.37) tends to American option under regime switching and rational exercise, as it was shown in [50] for American put option without regime switching.

Example 4.2.1. Let us consider a two regime model with the following parameters (see Example 1 in [70]):

$\boldsymbol{r}=\left(\begin{array}{l}r_{1} \\ r_{2}\end{array}\right)=\left(\begin{array}{c}0.1 \\ 0.05\end{array}\right), \boldsymbol{\sigma}=\left(\begin{array}{l}\sigma_{1} \\ \sigma_{2}\end{array}\right)=\left(\begin{array}{c}0.8 \\ 0.3\end{array}\right), Q=\left(\begin{array}{rr}-6 & 6 \\ 9 & -9\end{array}\right), T=1, E=9$.

In Table 4.7 the results of numerical solution by the proposed explicit scheme at the point $S=E$ with various intensity functions are collected for rationality parameter $\lambda$ from 1 to 1000 . The results show that the solution tends to American option price as $\lambda \rightarrow \infty$. We compare the results with other known techniques, such as front-fixing method, proposed for regime switching model in [43] and the tree method proposed in [87]. The obtained results for the fully implicit scheme $(\theta=1)$ and Crank-Nicolson method $\left(\theta=\frac{1}{2}\right)$ have not been shown in Table 4.7 , because they are very close to the those obtained with the explicit scheme. As one can see in this Table, the difference between the results of applying various intensity functions vanishes for the large enough rationality parameter. The intensity function family $f_{1}$ that corresponds to the penalty method, as well as its smooth analogue $f_{3}$ are convenient for the American option pricing problem due to their stability properties shown in [25]. 


\begin{tabular}{|c|cc|cc|ccc|}
\hline & \multicolumn{2}{|c|}{$f_{1}$} & \multicolumn{2}{|c|}{$f_{2}$} & \multicolumn{2}{c|}{$f_{3}$} \\
\hline$\lambda$ & Regime 1 & Regime 2 & Regime 1 & Regime 2 & Regime 1 & Regime 2 \\
\hline 1 & 1.9060 & 1.8229 & 1.6472 & 1.5407 & 1.6592 & 1.5532 \\
10 & 1.9545 & 1.8656 & 1.9596 & 1.8705 & 1.9150 & 1.8240 \\
100 & 1.9700 & 1.8805 & 1.9710 & 1.8815 & 1.9661 & 1.8765 \\
1000 & 1.9718 & 1.8819 & 1.9719 & 1.8819 & 1.9714 & 1.8819 \\
10000 & 1.9720 & 1.8820 & 1.9721 & 1.8820 & 1.9720 & 1.8820 \\
\hline FF & 1.9713 & 1.8817 & 1.9713 & 1.8817 & 1.9713 & 1.8817 \\
Tree & 1.9722 & 1.8819 & 1.9722 & 1.8819 & 1.9722 & 1.8819 \\
\hline
\end{tabular}

Table 4.7: Convergence of the solution for various intensity functions $f_{1}, f_{2}, f_{3}$ to American option price and comparison with front-fixing (FF) and Tree methods. The tests are done with explicit scheme $(\theta=0), h=10^{-2}$ and time step $k=10^{-4}$. 
In Figure 4.8 the option price at $\tau=T$ is presented for the a two regimes model when using the proposed explicit $(\theta=0)$ method. The intensity function is taken in the form 4.6) with rationality parameter $\lambda=10^{3}$. In this example, the step sizes $h=10^{-2}, k=10^{-4}$ have been chosen. In Figure 4.9 we present the solution of the problem with the set of parameters 4.79 ) and the matrix $Q$ given by

$$
Q=\left(\begin{array}{rr}
-1 & 1 \\
1 & -1
\end{array}\right)
$$

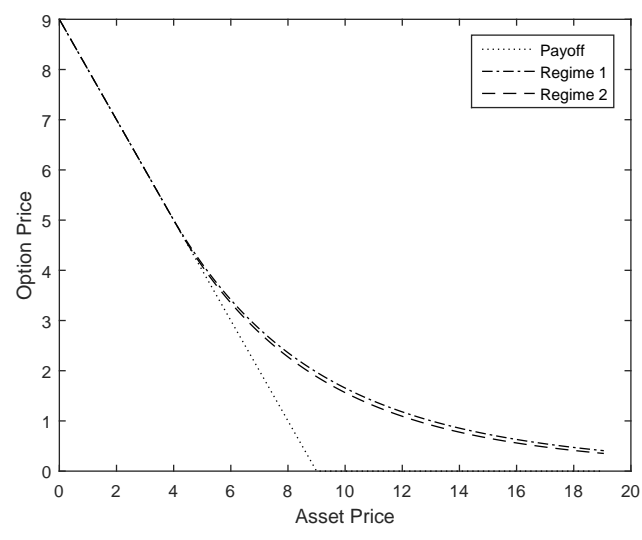

Figure 4.8: Numerical solution of the problem with parameters 4.79 )

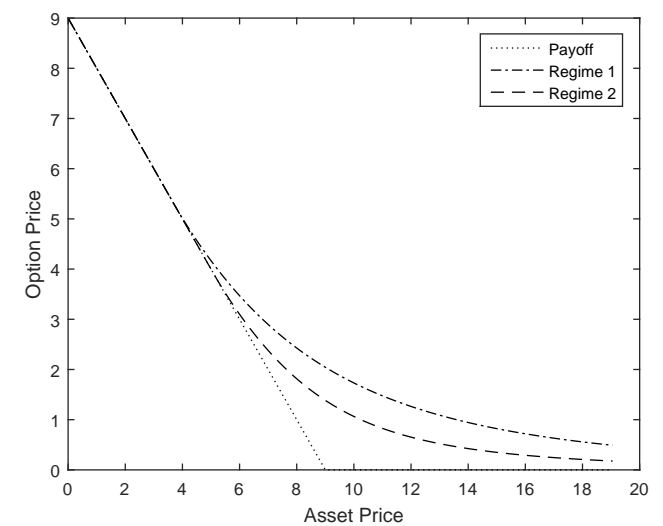

Figure 4.9: Numerical solution of the problem with parameters 4.79) with matrix 4.80

In Figures 4.10 and 4.11 the numerical solution with two different values of rationality parameter $\lambda=1$ and $\lambda=10^{3}$ is presented.

In the next example the numerical convergence rates of the proposed method for various families of intensity functions and rationality parameters are presented. Thus, numerical results for the fully implicit $(\theta=1)$, Crank-Nicolson $(\theta=1 / 2)$ and explicit $(\theta=0)$ schemes in the differential part of the PDE are shown.

Example 4.2.2. Let us consider the problem (4.41) with parameters (4.79). The experiments have been implemented for the fully implicit $(\theta=1)$, Crank-Nicolson $(\theta=1 / 2)$ and explicit $(\theta=0)$ schemes in the differential part of the PDE.

For this purpose, we have obtained a series of numerical results with fixed time step $k=2 \cdot 10^{-5}$ and the spatial steps $h=2 \cdot 10^{-2}, h / 2=10^{-2}$ and $h / 4=5 \cdot 10^{-3}$. The convergence rate $\gamma_{h}$ has been calculated by formula $(1.22)$ for the proposed 


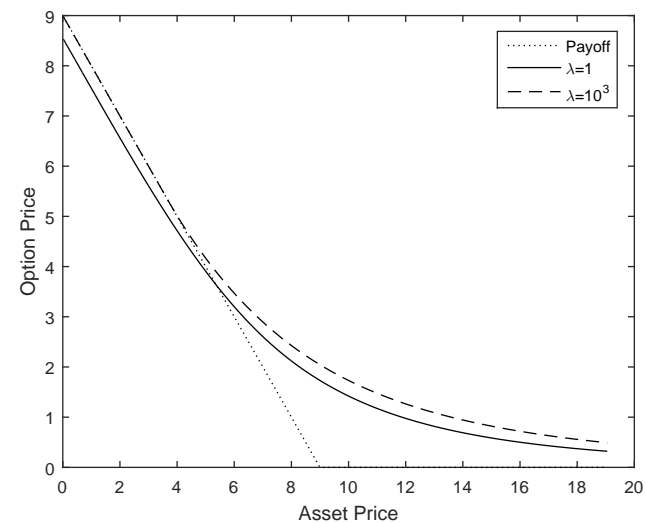

Figure 4.10: Numerical solution of the problem with parameters (4.79) with various $\lambda$ (Regime 1).

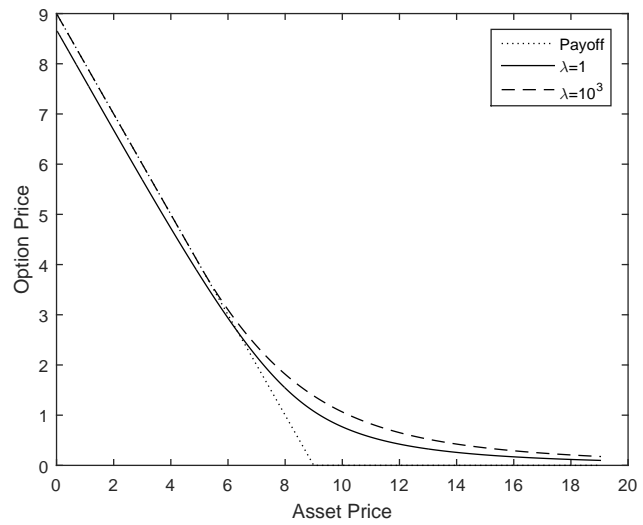

Figure 4.11: Numerical solution of the problem with parameters 4.79) with various $\lambda$ (Regime 2).

\begin{tabular}{|c|ccc|ccc|}
\hline & \multicolumn{3}{|c|}{ Regime 1 } & \multicolumn{3}{c|}{ Regime 2 } \\
$\theta$ & 0 & 0.5 & 1 & 0 & 0.5 & 1 \\
\hline$f_{1}$ & 2.0084 & 2.0003 & 2.0007 & 2.0143 & 2.0004 & 2.0015 \\
$f_{2}$ & 2.0083 & 2.0003 & 2.0005 & 2.0142 & 2.0007 & 2.0013 \\
$f_{3}$ & 2.0079 & 2.0002 & 2.0001 & 2.0156 & 2.0005 & 2.0004 \\
\hline
\end{tabular}

Table 4.8: Convergence rate in space of the proposed $\theta$-scheme for $\lambda=10^{3}$.

scheme with $\theta=0,0.5,1$. The results are collected in Table 4.8 showing the expected orders for the approximation with $\lambda=10^{3}$ and various intensity function families.

Analogous formula can be used in order to estimate the convergence rate in time, $\gamma_{k}$, for a fixed space step $h$ :

$$
\gamma_{k}=\log _{2} \frac{\left\|U_{k / 2}-U_{k}\right\|}{\left\|U_{k / 4}-U_{k / 2}\right\|} .
$$

In this case, the spatial step has been fixed to $h=5 \cdot 10^{-3}$, while the chosen time steps are $k=2 \cdot 10^{-5}, k / 2=10^{-5}, k / 4=5 \cdot 10^{-6}$. The convergence rates $\gamma_{k}$ of the proposed method for various intensity function families (4.4)-(4.6) are presented in Table 4.9. The numerical convergence rate are in agreement with the theoretical study of consistency developed in Section 4.2.2. 


\section{BEHAVIOURAL MODELLING OF OPTION PRICING}

\begin{tabular}{|c|ccc|ccc|}
\hline & \multicolumn{3}{|c|}{ Regime 1 } & \multicolumn{3}{c|}{ Regime 2 } \\
\hline$\theta$ & 0 & 0.5 & 1 & 0 & 0.5 & 1 \\
\hline$f_{1}$ & 1.0013 & 1.7795 & 1.0007 & 1.0013 & 1.8889 & 1.0010 \\
\hline$f_{2}$ & 1.0009 & 1.7802 & 1.0007 & 1.0009 & 1.9017 & 1.0007 \\
\hline$f_{3}$ & 1.0010 & 1.8543 & 1.0001 & 1.0010 & 1.8943 & 1.0000 \\
\hline
\end{tabular}

Table 4.9: Convergence rate in time of the proposed $\theta$-scheme for $\lambda=10^{3}$.

In the previous section the stability conditions for the proposed weighted scheme have been found. The forthcoming numerical example shows that the stability condition is crucial.

Example 4.2.3. Let us consider the explicit finite difference scheme (4.45) for the problem with parameters (4.79) in order to check the stability condition (4.54).

Figures 4.12 - 4.13 show the numerical solution for regime 1 and 2 respectively. More precisely, by taking a fixed value of $h=10^{-2}$ dashed lines show that the numerical solution is stable for $k=10^{-4}$, when the conditions 4.54) are fulfilled, while when $k=1.56 \cdot 10^{-4}$, the spurious oscillations occur as the scheme is not stable, thus leading to inaccurate numerical approximations that are shown in the solid line.

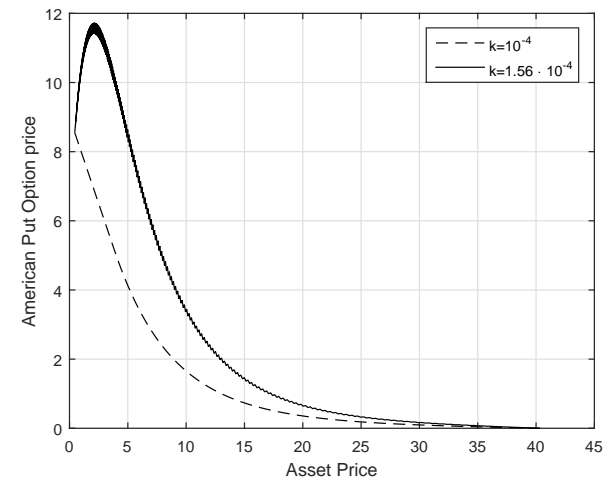

Figure 4.12: Stable and not stable solutions (Regime 1).

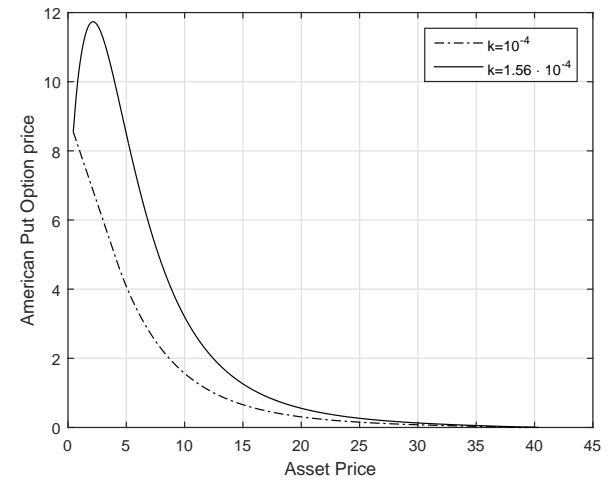

Figure 4.13: Stable and not stable solutions (Regime 2).

The results of this section have been published in [27]. 


\section{Valuation of multi-asset options}

Multiasset option pricing has two main challenges arising from their high dimensions and the correlations between assets. Probably the most popular methods are those around Monte Carlo method and its modifications [12], [64]. However, their simulation used to be time consuming and slow convergent [67].

Analytical-numerical methods like it occurs in one asset case are also existing in literature. So, Kirk and Aron [73] provide an approximation method to price a bivariate spread option problem, but it is inaccurate when the strike prices are high [1], [19]. Alexander improves Kirk's approach based on the hypothesis that the spread option price is the sum of the prices of two compound exchange options that avoids any strike convention.

Fourier Transform Approach and numerical integration techniques are used by J. Ziveyi and C. Chiarella in [23], [118] for numerical evaluation of American Options written on two underlying assets.

Among the numerical methods we mention the so-called lattice methods [13] and recently [67] that improves the previous ones and gives fast and accurate results although requires some minor correlation restrictions.

Dealing with pure finite difference methods the existence of the cross-derivative term in the PDE problem due to asset correlation, introduces additional challenges in the numerical solution due to the existence of negative coefficient terms in the 


\section{VALUATION OF MULTI-ASSET OPTIONS}

numerical scheme as well as involving expensive stencil schemes. Both facts may produce numerical drawbacks coming from the dominance of the connection versus the diffusion as well as more expensive computational cost, [26], [97], [105].

In order to overcome these difficulties dealing with finite difference approach, authors use recently several strategies. High order compact schemes are proposed in [40] using central difference approximations for stochastic volatility Heston model. The authors in [61] use ADI methods and clever discretizations to obtain stable numerical schemes under minor coefficients restrictions in the PDE. The authors of [22] and [105] use special finite difference approximations of the crossderivative term based on a seven points stencil instead of the nine points stencil scheme resulting from the central finite difference approach.

Finally, in our work we continue the removing cross-derivative approach based on the transformation of the PDE problem initiated in [26] for the case of one asset stochastic volatility Heston model and applied to the Bates stochastic volatility jump diffusion model in [47]. Apart from avoiding negative coefficients in the proposed scheme, it has the additional computational advantage that uses only a five point stencil.

In this chapter we consider multi-asset option pricing problems for both European and American cases. In further sections exchange, spread and basket options are studied. The general Black-Scholes equation for all these types of option takes the following form

$$
\begin{aligned}
\frac{\partial U}{\partial \tau}= & \frac{1}{2} \sigma_{1}^{2} S_{1}^{2} \frac{\partial^{2} U}{\partial S_{1}^{2}}+\frac{1}{2} \sigma_{2}^{2} S_{2}^{2} \frac{\partial^{2} U}{\partial S_{2}^{2}}+\rho \sigma_{1} \sigma_{2} S_{1} S_{2} \frac{\partial^{2} U}{\partial S_{1} \partial S_{2}} \\
& +\left(r-q_{1}\right) S_{1} \frac{\partial U}{\partial S_{1}}+\left(r-q_{2}\right) S_{2} \frac{\partial U}{\partial S_{2}}-r U
\end{aligned}
$$

The type of option is defined by the initial condition (payoff function) and appropriate boundary conditions. Some types of two asset American Option problems, such as Currency translated option (see [104]), American Better-of option, Exchange option (see [86]) and others, can be transformed into a one-spatial dimensional equation by a suitable change of variables. It is important to realize that a reduction in the number of dimensions can contribute greatly to efficiency of the finite difference implementation. After appropriate transformation we obtain the Black-Scholes equation for one-asset option. The study of multi-asset options starts with this case. 


\subsection{Exchange options}

\subsection{Exchange options}

An American Exchange Option (AEO) is an option which gives the holder the right to exchange one asset to another at any time prior the expiration date $T$. Let $S_{1}$ and $S_{2}$ be a price of asset 1 and asset 2 (see [86]). The payoff function is defined as follows

$$
F\left(S_{1}, S_{2}\right)=\max \left(S_{1}-S_{2}, 0\right) .
$$

At time $\tau=T-t$ American option price $P\left(S_{1}, S_{2}, \tau\right)$ for $P>\max \left(S_{1}-S_{2}, 0\right)$ satisfies the two-dimensional equation (5.1).

We introduce the notation $X_{f}(\tau)$ to represent the moving boundary such as for any $S_{1}, S_{2}: \frac{S_{1}}{S_{2}}<X_{f}(\tau)$ at time $\tau=T-t$ American exchange option price $U\left(S_{1}, S_{2}, \tau\right)$ satisfies the multi-dimensional equation (5.1).

To have a smooth transition we also require that the gradient of the option value (with respect to the underlying asset prices) is continuous at the boundary. This socalled smooth pasting condition is given by

$$
\begin{gathered}
\left.U\left(S_{1}, S_{2}, \tau\right)\right|_{X_{f}(\tau)}=S_{1}-S_{2}, \\
\left.\frac{\partial U}{\partial S_{1}}\right|_{X_{f}(\tau)}=1,\left.\quad \frac{\partial U}{\partial S_{2}}\right|_{X_{f}(\tau)}=-1 .
\end{gathered}
$$

On the other boundaries the following conditions are established

$$
U\left(0, S_{2}, \tau\right)=0, \quad U\left(S_{1}, 0, \tau\right)=S_{1}
$$

We consider only hold region $H(\tau)=\left\{\left(S_{1}, S_{2}\right): \frac{S_{1}}{S_{2}}<X_{f}(\tau)\right\}$. There is an opportunity to reduce the dimension of the problem by the transformation

$$
\xi=\frac{S_{1}}{S_{2}}, \quad p(x, \tau)=\frac{U\left(S_{1}, S_{2}, \tau\right)}{S_{2}} .
$$

Then the AEO pricing problem reduces to one dimensional American call option price problem

$$
\frac{\partial p}{\partial \tau}=\frac{\sigma^{2}}{2} \xi^{2} \frac{\partial^{2} p}{\partial \xi^{2}}+(r-q) \xi \frac{\partial p}{\partial \xi}-r p, \quad \xi<X_{f}(\tau), \quad 0<\tau<T,
$$

where

$$
\sigma^{2}=\sigma_{1}^{2}-2 \rho \sigma_{1} \sigma_{2}+\sigma_{2}^{2}, \quad r=q_{2}, \quad q=q_{1},
$$



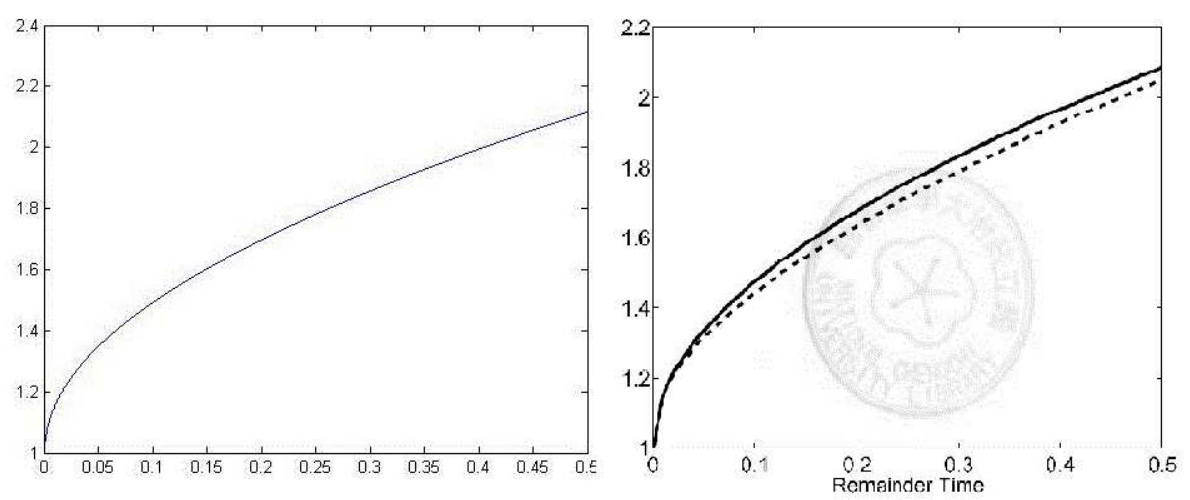

Figure 5.1: Optimal exercise ratio in time: calculated by the proposed in Section 2.2 method (left) and presented in [86].

with the initial and boundary conditions

$$
\begin{gathered}
p(\xi, 0)=\max (\xi-1,0), \quad p(0, \tau)=0 . \\
p\left(X_{f}(\tau), \tau\right)=X_{f}(\tau)-1,\left.\quad \frac{\partial p}{\partial \xi}\right|_{\xi=X_{f}(\tau)}=1,
\end{gathered}
$$

Then one can apply the front-fixing transformation presented in Section 2.2 to (5.2) $-(5.5)$.

As a numerical example, we consider the American exchange option for correlated assets with $\rho=0.5$, and $\sigma_{1}=\sigma_{2}=0.5$, then $\sigma^{2}=0.25$ by (5.3). The results are presented on Figure 5.1 .

Further we consider such types of multi-asset options that the dimension reduction is not applicable. As it was mentioned above, we use cross-derivative elimination technique in order to improve qualitative properties of the numerical scheme. 


\subsection{Spread options}

In this section we consider spread option pricing problems for both European and American cases. Firstly, we deal with European spread call option price $U\left(S_{1}, S_{2}, \tau\right)$ that is the solution of the PDE (5.1) with the initial condition

$$
U\left(S_{1}, S_{2}, 0\right)=g\left(S_{1}, S_{2}\right)=\left(S_{1}-S_{2}-E\right)^{+} .
$$

Spread options are frequently traded in the energy market [19]. Two examples are:

Crack spreads: Options on the spread between refined petroleum products and crude oil. The spread represents the refinement margin made by the oil refinery by "cracking" the crude oil into a refined petroleum product.

Spark spreads: Options on the spread between electricity and some type of fuel. The spread represents the margin of the power plant, which takes fuel to run its generator to produce electricity. The case $E=0$ corresponds to exchange options that can be reduce to $1 \mathrm{D}$ problem as described above.

Since the numerical solution of European multi-asset options requires the selection of a bounded numerical domain and the translation of the boundary conditions to the boundary of the domain, it is important to pay attention to such conditions. For the initial problem (5.1) coupled with (5.6) suitable boundary conditions are:

1. For $S_{1}=0$ the payoff $\left(S_{1}-S_{2}-E\right)^{+}$suggests the Dirichlet's condition

$$
U\left(0, S_{2}, \tau\right)=0, \quad 0<S_{2}<\infty, \quad 0<\tau \leq T
$$

as one dimensional call option.

2. Taking the ideas developed by some authors, for instance D. Duffy for the case of basket option (see [37], p. 270), for $S_{2}=0$ we take the value of the closed form solution of the basic Black-Scholes equation of a call for $S_{1}$ with given strike $E$,

$$
U\left(S_{1}, 0, \tau\right)=e^{-r \tau}\left(F N\left(\phi_{1}\right)-E N\left(\phi_{2}\right)\right)
$$

where

$$
F=S_{1} e^{\left(r-q_{1}\right) \tau}, \quad \phi_{1}=\frac{1}{\sigma_{1} \sqrt{\tau}}\left[\log \frac{F}{E}+\frac{1}{2} \sigma_{1}^{2} \tau\right], \quad \phi_{2}=\phi_{1}-\sigma_{1} \sqrt{\tau},
$$

where $N(x)$ is the standard normal cumulative distribution function. 
3. As $S_{1}$ is large versus $S_{2}+E$, then the behaviour of the solution looks like the asymptotic value of a one dimensional vanilla call option with the strike $\left(S_{2}+E\right)$ for $S_{1} \gg S_{2}+E$ (see [100], p. 157),

$$
U\left(S_{1}, S_{2}, \tau\right) \approx e^{-q_{1} \tau} S_{1}-e^{-r \tau}\left(S_{2}+E\right), \quad S_{1} \gg S_{2}+E
$$

4. For large values of $S_{2}$ we assume that the values are almost constants when $S_{2}$ changes, therefore we can use Neuman's boundary condition:

$$
\frac{\partial U}{\partial S_{2}}=0
$$

These boundary conditions will be validated with the numerical examples.

\subsubsection{Removing the cross-derivative term}

We begin the study by transforming the PDE problem (5.1) in order to remove the cross-derivative term. Firstly we eliminate the reaction term $r U$ by means of the substitution

$$
V=e^{r \tau} U
$$

obtaining

$$
\begin{aligned}
\frac{\partial V}{\partial t}= & \frac{1}{2} \sigma_{1}^{2} S_{1}^{2} \frac{\partial^{2} V}{\partial S_{1}^{2}}+\frac{1}{2} \sigma_{2}^{2} S_{2}^{2} \frac{\partial^{2} V}{\partial S_{2}^{2}}+\rho \sigma_{1} \sigma_{2} S_{1} S_{2} \frac{\partial^{2} V}{\partial S_{1} \partial S_{2}} \\
& +\left(r-q_{1}\right) S_{1} \frac{\partial V}{\partial S_{1}}+\left(r-q_{2}\right) S_{2} \frac{\partial V}{\partial S_{2}} .
\end{aligned}
$$

In order to remove the cross derivative term, note that the right-hand side of 5.13 , is a linear differential operator of two variables and classical techniques to obtain the canonical form of second order linear PDEs can be applied, see for instance chapter 3 of [51]. Under the assumption of correlated variables with $-1<\rho<1$ the sign of the discriminant is

$$
a_{12}^{2}-4 a_{11} a_{22}=\sigma_{1}^{2} \sigma_{2}^{2} S_{1}^{2} S_{2}^{2}\left(\rho^{2}-1\right)<0,
$$

where

$$
a_{11}=\frac{1}{2} \sigma_{1}^{2} S_{1}^{2} ; \quad a_{12}=\rho \sigma_{1} \sigma_{2} S_{1} S_{2} ; \quad a_{22}=\frac{1}{2} \sigma_{2}^{2} S_{2}^{2}
$$


Therefore the right-hand side of equation (5.13) becomes of elliptic type and a convenient substitution to obtain the canonical form is given by solving the ordinary differential equation

$$
\frac{d S_{2}}{d S_{1}}+\frac{\sigma_{2} S_{2}}{\sigma_{1} S_{1}}(-\rho \pm i \tilde{\rho})=0, \quad \tilde{\rho}=\sqrt{1-\rho^{2}},
$$

and $\frac{\sigma_{2} S_{2}}{\sigma_{1} S_{1}}(-\rho \pm i \tilde{\rho})$ are the conjugate roots of $a_{11} x^{2}+a_{12} x+a_{22}=0$. Solving (5.14) one gets

$$
\frac{1}{\sigma_{2}} \log S_{2}+\frac{-\rho \pm i \tilde{\rho}}{\sigma_{1}} \log S_{1}=C_{0},
$$

where one can relate the integration constant $C_{0}$ to the new variables by

$$
x=\operatorname{Im}\left(C_{0}\right), \quad y=-\operatorname{Re}\left(C_{0}\right) .
$$

From (5.15) and (5.16) it follows the expression of the new variables

$$
x=\frac{\tilde{\rho}}{\sigma_{1}} \log S_{1} ; \quad y=\frac{\rho}{\sigma_{1}} \log S_{1}-\frac{1}{\sigma_{2}} \log S_{2} .
$$

Note that

$$
y-m x=-\frac{1}{\sigma_{2}} \log S_{2}, \quad m=\frac{\rho}{\tilde{\rho}} .
$$

By denoting

$$
W(x, y, \tau)=V\left(S_{1}, S_{2}, \tau\right),
$$

equation (5.13) takes the following form without cross derivative term for $(x, y) \in$ $\mathbb{R}^{2}, 0<\tau \leq T$,

$$
\begin{aligned}
\frac{\partial W}{\partial \tau}= & \frac{\tilde{\rho}^{2}}{2}\left(\frac{\partial^{2} W}{\partial x^{2}}+\frac{\partial^{2} W}{\partial y^{2}}\right)+\frac{\tilde{\rho}}{\sigma_{1}}\left(r-q_{1}-\frac{\sigma_{1}^{2}}{2}\right) \frac{\partial W}{\partial x} \\
& +\left(\frac{\rho}{\sigma_{1}}\left(r-q_{1}-\frac{\sigma_{1}^{2}}{2}\right)-\frac{1}{\sigma_{2}}\left(r-q_{2}-\frac{\sigma_{2}^{2}}{2}\right)\right) \frac{\partial W}{\partial y} .
\end{aligned}
$$

In accordance with [44], [66] and equation (5.10) we choose the rectangular domain $\left(S_{1}, S_{2}\right) \in\left[a_{1}, b_{1}\right] \times\left[a_{2}, b_{2}\right]=\Omega$, where $a_{i}>0, i=1,2$ are small positive values; $b_{2}$ about $3 E$ and $b_{1}$ about $3\left(b_{2}+E\right)$. For the transformed problem (5.20) due to 5.17 , the rectangular domain $\Omega$ becomes rhomboid with vertices $A B C D$ (see Figure 5.2). From 5.17) let us denote

$$
c_{1}=\frac{\tilde{\rho}}{\sigma_{1}} \log a_{1}, \quad c_{2}=\frac{\log a_{2}}{\sigma_{2}}, \quad d_{1}=\frac{\tilde{\rho}}{\sigma_{1}} \log b_{1}, \quad d_{2}=\frac{\log b_{2}}{\sigma_{2}} .
$$


By (5.18) rhomboid domain has the sides:

$$
\begin{aligned}
& \overline{A D}=\left\{(x, y): \quad x=c_{1}, m c_{1}-d_{2} \leq y \leq m c_{1}-c_{2}\right\} \\
& \overline{A B}=\left\{(x, y): \quad c_{1} \leq x \leq d_{1}, y=m x-d_{2}\right\} \\
& \overline{B C}=\left\{(x, y): \quad x=d_{1}, m d_{1}-d_{2} \leq y \leq m d_{1}-c_{2}\right\} \\
& \overline{C D}=\left\{(x, y): \quad c_{1} \leq x \leq d_{1}, y=m x-c_{2}\right\} .
\end{aligned}
$$

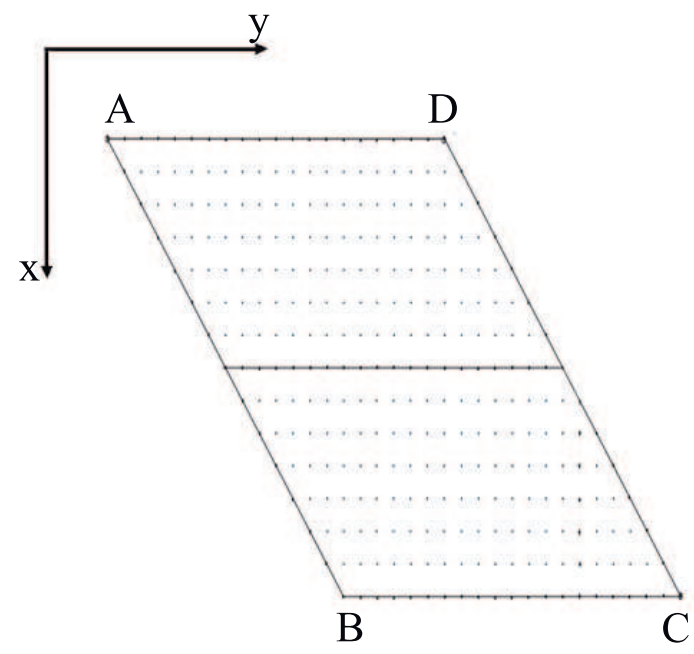

Figure 5.2: Numerical domain after removing cross derivative term transformation.

The mesh points in the rhomboid domain are $\left(x_{i}, y_{j}\right)$ such that

$$
\begin{gathered}
x_{i} \in\left[c_{1}, d_{1}\right], \quad x_{i}=c_{1}+i h, \quad 0 \leq i \leq N_{x}, \quad \Delta_{x}=h=\frac{d_{1}-c_{1}}{N_{x}}, \\
\Delta_{y}=|m| h, \quad N_{y}=\frac{d_{2}-c_{2}}{|m| h}, \quad y_{j}=m x_{i}-d_{2}+(j-i)|m| h, i \leq j \leq N_{y}+i .
\end{gathered}
$$

The approximations of the derivatives appearing in 5.20 at the point $\left(x_{i}, y_{j}, \tau^{n}\right)$ are the following

$$
\begin{array}{cc}
\frac{\partial W}{\partial x} \sim \frac{w_{i+1, j}^{n}-w_{i-1, j}^{n}}{2 h}, & \frac{\partial W}{\partial y} \sim \frac{w_{i, j+1}^{n}-w_{i, j-1}^{n}}{2|m| h}, \\
\frac{\partial^{2} W}{\partial x^{2}} \sim \frac{w_{i+1, j}^{n}-2 w_{i, j}^{n}+w_{i-1, j}^{n}}{h^{2}}, & \frac{\partial^{2} W}{\partial y^{2}} \sim \frac{w_{i, j+1}^{n}-2 w_{i, j}^{n}+w_{i, j-1}^{n}}{m^{2} h^{2}},
\end{array}
$$




$$
\frac{\partial W}{\partial \tau} \sim \frac{w_{i, j}^{n+1}-w_{i, j}^{n}}{k}
$$

where $w_{i, j}^{n} \sim W\left(x_{i}, y_{j}, \tau^{n}\right), \tau^{n}=n k$.

Substituting these finite difference approximations of the derivatives the equation (5.17) is approximated by the explicit difference scheme with five points stencil

$$
w_{i, j}^{n+1}=\alpha_{1} w_{i-1, j}^{n}+\alpha_{2} w_{i+1, j}^{n}+\alpha_{3} w_{i, j}^{n}+\alpha_{4} w_{i, j-1}^{n}+\alpha_{5} w_{i, j+1}^{n},
$$

where

$$
\begin{aligned}
\alpha_{1,2}= & \frac{\tilde{\rho}^{2}}{2} \frac{k}{h^{2}} \mp \frac{\tilde{\rho}}{\sigma_{1}}\left(r-q_{1}-\frac{\sigma_{1}^{2}}{2}\right) \frac{k}{2 h}, \\
\alpha_{3}= & 1-\tilde{\rho}^{2} \frac{k}{h^{2}}\left(1+\frac{1}{m^{2}}\right), \\
\alpha_{4,5}= & \frac{\tilde{\rho}^{2}}{2} \frac{k}{m^{2} h^{2}} \mp \\
& {\left[\frac{\tilde{\rho}}{\sigma_{1}}\left(r-q_{1}-\frac{\sigma_{1}^{2}}{2}\right)-\frac{\tilde{1}}{\sigma_{2}}\left(r-q_{2}-\frac{\sigma_{2}^{2}}{2}\right)\right] \frac{k}{2|m| h} . }
\end{aligned}
$$

The discretization of the spacial boundary is given by

$$
\begin{aligned}
& P(\overline{A D})=\left\{\left(x_{0}, y_{j}\right): 0 \leq j \leq N_{y}\right\}, \\
& P(\overline{A B})=\left\{\left(x_{i}, y_{i}\right): 0 \leq i \leq N_{x}\right\}, \\
& P(\overline{B C})=\left\{\left(x_{N_{x}}, y_{j}\right): N_{x} \leq j \leq N_{x}+N_{y}\right\}, \\
& P(\overline{C D})=\left\{\left(x_{i}, y_{N_{y}+i}\right): 0 \leq i \leq N_{x}\right\} .
\end{aligned}
$$

Both initial and boundary conditions (5.6), (5.7), (5.8), (5.10) and (5.11) are transformed throughout equations (5.12), (5.17), (5.19). For the initial condition we have

$$
w_{i, j}^{0}=\left(e^{\frac{\sigma_{1} x_{i}}{\tilde{\rho}}}-e^{-\sigma_{2}\left(y_{j}-m x_{i}\right)}-E\right)^{+}, \quad 0 \leq i \leq N_{x}, i \leq j \leq i+N_{x} .
$$

Boundary condition for side $\overline{A D}$ :

$$
w_{0, j}^{n}=0, \quad 0 \leq j \leq N_{y}, 1 \leq n \leq N_{\tau} .
$$

For $\overline{C D}$ with small value of $S_{2}$, we have transformed Black-Scholes solution, so that

$$
w_{i, N_{y}+i}^{n}=F_{i}^{n} N\left(\phi_{1, i}^{n}\right)-E N\left(\phi_{2, i}^{n}\right), \quad 0<i<N_{x}, 1 \leq n \leq N_{\tau},
$$


where

$$
\begin{gathered}
F_{i}^{n}=\exp \left(\frac{\sigma_{1}}{\tilde{\rho}} x_{i}+\left(r-q_{1}\right) \tau^{n}\right), \\
\phi_{1, i}^{n}=\frac{1}{\sigma_{1} \sqrt{\tau^{n}}}\left[\log \frac{F_{i}}{E}+\frac{1}{2} \sigma_{1}^{2} \tau^{n}\right], \quad \phi_{2, i}^{n}=\phi_{1, i}^{n}-\sigma_{1} \sqrt{\tau^{n}} .
\end{gathered}
$$

Side $\overline{B C}$ corresponds to large values of $S_{1}$, so behaviour of the value of option for $N_{x}+1 \leq j \leq N_{x}+N_{y}, 1 \leq n \leq N_{\tau}$ there leads to

$$
w_{N_{x}, j}^{n}=\exp \left(\frac{\sigma_{1}}{\tilde{\rho}} x_{N_{x}}+\left(r-q_{1}\right) \tau^{n}\right)-\left(\exp \left(\sigma_{2}\left(m x_{N_{x}}-y_{j}\right)\right)+E\right) .
$$

Boundary $\overline{A B}$ corresponds with the boundary for large values of $S_{2}$. Condition (5.11) means that component $S_{1}$ is constant and the null first derivative is approximated by the forward difference $\frac{w_{i, i+1}^{n}-w_{i, i}^{n}}{|m| h}=0$,

$$
w_{i, i}^{n}=w_{i, i+1}^{n}, \quad 1 \leq i \leq N_{x}, 1 \leq n \leq N_{\tau} .
$$

\subsubsection{Numerical analysis of the method}

In this section we show that the introduced numerical scheme (5.21) - (5.24) presents suitable qualitative and computational properties, such as consistency and conditional positivity and stability.

In order to guarantee positivity of the numerical solution let us check the positivity of the coefficients $\alpha_{i}$. Values in interior points of the numerical domain $w_{i, j}^{n+1}$, $1 \leq i \leq N_{x}-1 ; i+1 \leq j \leq N_{y}+i-1$, calculated by the scheme (5.21), preserve non-negativity from the previous time level $n$ at all interior and boundary points $w_{i, j}^{n} \geq 0,0 \leq i \leq N_{x} ; i \leq j \leq N_{y}+i$ under conditions

$$
\begin{gathered}
h<\min \left\{\frac{\tilde{\rho} \sigma_{1}}{\left|r-q_{1}-\frac{\sigma_{1}^{2}}{2}\right|} ; \frac{\tilde{\rho}^{2}}{\left|m\left[\frac{\tilde{\rho}}{\sigma_{1}}\left(r-q_{1}-\frac{\sigma_{1}^{2}}{2}\right)-\frac{1}{\sigma_{2}}\left(r-q_{2}-\frac{\sigma_{2}^{2}}{2}\right)\right]\right|}\right\} . \\
k<\frac{m^{2} h^{2}}{\tilde{\rho}^{2}\left(m^{2}+1\right)} .
\end{gathered}
$$

Let us pay attention now to the positivity of the numerical solution at the boundary of the numerical domain. On the boundary $\overline{A D}$ from 5.26 we have zero values. On the boundary $\overline{C D}$ the formula 5.27) is used preserving the positivity since it is the transformed Black-Scholes formula throughout the expression 
5.17. Along the line $\overline{A B}$ we use Neumann boundary condition 5.29. Therefore, the positivity at the neighbour interior point guarantees the positivity at the boundary. Finally, on the line $\overline{B C}$ boundary conditions are determined by the formula (5.28). This is transformed expression for the asymptotic behaviour of the call option 5.10 that is positive under the condition

$$
S_{1_{\max }}>\left(S_{2_{\max }}+E\right) \exp \left(\left(q_{1}-r\right) \tau^{n}\right), \quad 1 \leq n \leq N_{\tau} .
$$

Following the ideas of Kangro [66], the computational domain has to be large enough to translate the boundary conditions, therefore

$$
S_{1_{\max }} \geq \max \left\{\left(S_{2_{\max }}+E\right) \exp \left(\left(q_{1}-r\right) T\right), 3\left(S_{2_{\max }}+E\right)\right\} .
$$

Therefore, choosing $S_{1_{\max }}$ according to 5.32 for the transformed problem $w_{N_{x}, j}^{n} \geq 0$ for any $N_{x}+1 \leq j \leq N_{x}+N_{y}, 1 \leq n \leq N_{\tau}$.

Summarizing, the non-negativity of the numerical solution follows from the non-negativity of the initial values and positivity preservation under the scheme and boundary conditions. In order to study stability of the scheme let us find the maximum value of the $w_{i, j}^{n}$ with respect to $i, j$ for each fixed $n$. For $n=0$ the values $w_{i, j}^{n}$ are defined by the initial condition (5.25). Let us consider the following function

$$
f(x, y)=\exp \left(\frac{\sigma_{1}}{\tilde{\rho}} x\right)-\exp \left(-\sigma_{2}(y-m x)\right)-E,
$$

and find the maximum value on the domain $\left[c_{1}, d_{1}\right] \times\left[c_{2}, d_{2}\right]$. The first derivatives are

$$
\begin{gathered}
\frac{\partial f}{\partial x}=\frac{\sigma_{1}}{\tilde{\rho}} \exp \left(\frac{\sigma_{1}}{\tilde{\rho}} x\right)+\sigma_{2} m \exp \left(-\sigma_{2}(y-m x)\right)>0 \\
\frac{\partial f}{\partial y}=\sigma_{2} \exp \left(-\sigma_{2}(y-m x)\right)>0
\end{gathered}
$$

Analysing 5.34 and 5.35) one concludes that the function $f(x, y)$ is increasing with respect to $x$ and $y$. Therefore, the maximum value is reached at the point $\left(d_{1}, d_{2}\right)$ :

$$
\begin{aligned}
\max _{(x, y) \in\left[c_{1}, d_{1}\right] \times\left[c_{2}, d_{2}\right]} f(x, y) & =f\left(d_{1}, d_{2}\right)=\exp \left(\frac{\sigma_{1}}{\tilde{\rho}} d_{1}\right)-\exp \left(-\sigma_{2}\left(d_{2}-m d_{1}\right)\right)-E \\
& =C=\text { const } .
\end{aligned}
$$


For the next time levels $n \geq 1$ let us consider the numerical scheme (5.21). Under conditions (5.30) and (5.31) the coefficients $\alpha_{i}>0, i=1, \ldots, 5$. Moreover, it is easy to check that

$$
\sum_{i=1}^{5} \alpha_{i}=1
$$

Therefore the values in interior points at the $(n+1)$-th level, $n \geq 0$, can be bounded by the following

$$
w_{i, j}^{n+1} \leq \max \left\{w_{i-1, j}^{n}, w_{i+1, j}^{n}, w_{i, j}^{n}, w_{i, j-1}^{n}, w_{i, j+1}^{n}\right\} \leq \max _{i, j} w_{i, j}^{n} .
$$

This maximum can be reached on the boundary. Therefore, let us study the boundary conditions. From 5.26 one gets that

$$
\max _{\overline{A D}} w_{i, j}^{n+1}=0 \text {. }
$$

The values on the boundary $\overline{A B}$ are equal to the interior points, therefore the inequality (5.37) can be applied.

The values on the boundary $\overline{B C}$ are increasing with respect to the index $j$ and reach the maximum at the point $\left(N_{x}, N_{x}+N_{y}-1\right)$. From the other side, this value can be bounded by the value at the point $\left(N_{x}, N_{x}+N_{y}\right)$ that is calculated by the formula (5.27). From the theory of option pricing, the price of European call is increasing with respect to the asset price $S$ and, moreover, it is always greater than its asymptotic function (see [58], p. 268, formula (13.1)). The proposed transformation preserves monotonicity, therefore,

$$
\begin{aligned}
w_{N_{x}, N_{x}+N_{y}}^{n+1} & =F_{N_{x}}^{n+1} N\left(\alpha_{1, N_{x}}^{n+1}\right)-E N\left(\alpha_{2, N_{x}}^{n+1}\right) \\
& \geq e^{\left(\frac{\sigma_{1}}{\tilde{\rho}} x_{N_{x}}+\left(r-q_{1}\right) \tau^{n+1}\right)}-\left(e^{\left(\sigma_{2}\left(m x_{N_{x}}-y_{N_{x}+N_{y}-1}\right)\right)}+E\right)=w_{N_{x}, N_{x}+N_{y}-1}^{n+1} .
\end{aligned}
$$

Summarizing we can conclude that

$$
\max _{i, j} w_{i, j}^{n+1}=w_{N_{x}, N_{x}+N_{y}}^{n+1}
$$

This value is transformed call option price for the asset price $S_{1_{\max }}$, i.e. the solution of the $1 D$ Black-Scholes equation at the fixed point. European call option gives a right to the option holder to purchase one share stock for a certain price. The option itself cannot be worth more than the stock. In the other words,

$$
U\left(S_{1}, 0, \tau\right)=e^{-q_{1} \tau} S_{1} N\left(d_{1}\right)-e^{-r \tau} E N\left(d_{2}\right) \leq e^{-q \tau} S_{1} N\left(d_{1}\right) \leq e^{-q \tau} S_{1},
$$




$$
w_{N_{x}, N_{x}+N_{y}}^{n+1} \leq e^{r \tau^{n+1}} e^{-q_{1} \tau^{n+1}} S_{1_{\max }} \leq e^{\left|r-q_{1}\right| T} S_{1_{\max }}, \quad 0 \leq n \leq N_{\tau}-1 .
$$

Then the following result can be stated.

Theorem 5.2.1. With previous notations, under the conditions (5.30) and (5.31) the numerical scheme (5.21), (5.25)-(5.29) is conditionally uniformly $\|\cdot\|_{\infty}$-stable with upper bound $A=e^{\left|r-q_{1}\right| T} S_{1_{\max }}$.

The study of consistency gives the following result:

Theorem 5.2.2. Assuming that the solution of the PDE (5.20) admits two times continuous partial derivative with respect to time and up to order four with respect to each of space directions solution, the numerical solution computed by the scheme (5.21) is consistent with the equation (5.20) with the second order in spatial variables and with the first order in time.

\subsubsection{American spread options}

In the case of American type option the holder has the right to exercise option at any moment before expire. It leads to a free boundary problem [48]. In order to simplify the computational procedure this problem can be considered as a linear complementarity problem (LCP):

$$
\begin{gathered}
\left(\frac{\partial U}{\partial \tau}-L U\right)(U-g)=0 \\
\frac{\partial U}{\partial \tau}-L U \geq 0, \quad U-g\left(S_{1}, S_{2}\right) \geq 0,
\end{gathered}
$$

where $L$ represents the spatial differential operator of the equation (5.1).

If we rewrite equation 5.20 in the form

$$
\frac{\partial W}{\partial \tau}-\mathcal{L} W=0
$$

then under the transformation (5.17) LCP 5.38)-(5.39) has the following form

$$
\left(\frac{\partial W}{\partial \tau}-\mathcal{L} W\right)(W-f(x, y))=0
$$




$$
\frac{\partial W}{\partial \tau}-\mathcal{L} W \geq 0 ; \quad W-f(x, y) \geq 0,
$$

being $\mathcal{L}$ the right hand side of the equation (5.20) and $f(x, y)$ is given by 5.33 .

Numerical solution for the problem (5.40)-(5.41) can be easily obtained by a small modification of the calculating procedure described above in Sections 2 and 4. On each time level

$$
\tilde{w}_{i, j}^{n}=\max \left\{w_{i, j}^{n}, f\left(x_{i}, y_{j}\right)\right\} \geq 0,
$$

where $w_{i, j}^{n}$ is defined by the finite difference equation (5.21).

\subsubsection{Numerical examples}

In this section we illustrate numerical result for both European and American spread options.

In Example 5.2.1 we show that the stability conditions 5.30) and 5.31) for the European spread option can not be disregarded.

Example 5.2.1. We tested the algorithm for the European spread call option pricing problem with the parameters:

$$
T=1 ; E=100 ; \sigma_{1}=0.2 ; \sigma_{2}=0.1 ; r=0.1 ; q_{1}=0.01 ; q_{2}=0.05 ; \rho=0.1 ;
$$

with chosen large enough $S_{2_{\max }}=400, S_{1_{\max }}=3\left(S_{2_{\max }}+E\right)=1500$.

The stability conditions (5.30), 5.31) on space and time steps are as follows

$$
h<\min \{2.8428 ; 23.7358\}=2.8428, \quad k<0.0112 \text {. }
$$

These conditions are crucial for the qualitative properties such as positivity and stability of the scheme. In Figure 5.3 the numerical solution with $h$ and $k$ satisfying (5.43) is presented. On the other hand, in Figure 5.4 there is a numerical simulation with time step $k=0.012>0.0112$. The solution is unstable and it has negative values.

The next Example 5.2.2 illustrates the computational time as well as the comparison with analytical approximation, provided by [1].

Example 5.2.2. Consider the European spread option with the data of Example 5.2.1 and choosing step sizes satisfying the stability condition. 


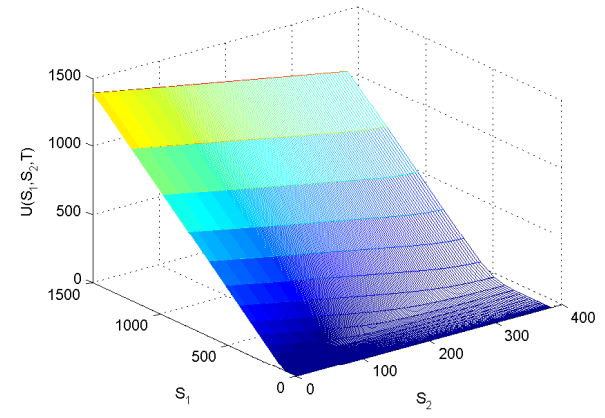

Figure 5.3: European spread option (stable), condition $(5.43)$ is fulfilled.

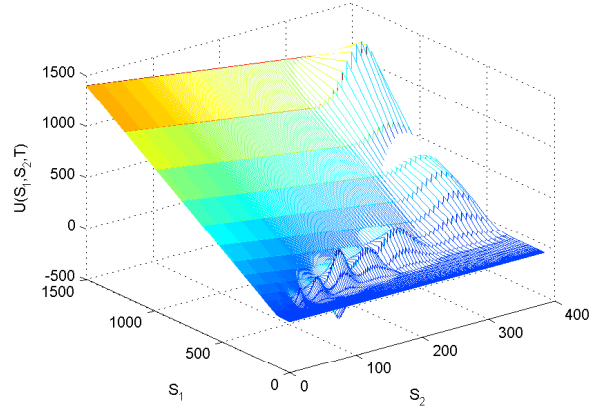

Figure 5.4: European spread option price, condition $(5.43)$ is broken.

The price of European spread option can be expressed as the price of a compound exchange option (see [1]). The known derived analytical approximation for the spread option reads

$$
U\left(S_{1}, S_{2}, T\right)=S_{1} e^{-q_{1} T} N\left(d_{1}\right)-\left(E e^{-r T}+S_{2}\right) e^{-\left(r-\tilde{r}-\tilde{q}_{2}\right) T} N\left(d_{2}\right)
$$

where

$$
\begin{gathered}
d_{1}=\frac{1}{\sigma \sqrt{T}}\left(\ln \frac{S_{1}}{S_{2}+E e^{-r T}}+\left(r-\tilde{r}+\tilde{q}_{2}-q_{1}+\frac{\sigma^{2}}{2}\right) T\right), \quad d_{2}=d_{1}-\sigma \sqrt{T}, \\
\sigma=\sqrt{\sigma_{1}^{2}-2 \rho \sigma_{1} \sigma_{2} \frac{S_{2}}{S_{2}+E e^{-r T}}+\left(\frac{S_{2}}{S_{2}+E e^{-r T}}\right)^{2} \sigma_{2}^{2},} \\
\tilde{r}=\frac{S_{2}}{S_{2}+E e^{-r T}} r, \quad \tilde{q}_{2}=\frac{S_{2}}{S_{2}+E e^{-r T}} q_{2} .
\end{gathered}
$$

The comparison of our proposed numerical method and the analytical approximation (5.44) is presented in Table 5.1. Analytical approximation is calculated for the same arrays of $S_{1}$ and $S_{2}$ using MATLAB-function $c d f$ for calculating cumulative distribution function, that requires a lot of computational resources, for each point. In the proposed method $c d f$ is used only for the boundary conditions. Last row in the Table 1 presents the CPU-time for each method for the same sizes of array. For approximation method $c d f$-time is $925.018 \mathrm{sec}$. It is the main part of computational time. In the case of FDM $c d f$ takes less than half of computational time - $48.867 \mathrm{sec}$. 


\begin{tabular}{|c|c|c|c|}
\hline$S_{2}$ & $S_{1}$ & FDM & Approximation \\
\hline 100 & 400 & 210.5978 & 209.7261 \\
& 200 & 24.2895 & 23.4543 \\
& 100 & 0.1150 & 0.0123 \\
& 50 & $3.1179 e-05$ & $-4.7741 e-10$ \\
\hline 90 & 400 & 220.0678 & 220.1714 \\
& 200 & 29.8745 & 29.8724 \\
& 100 & 0.1691 & 0.0372 \\
& 50 & $5.2150 e-05$ & $-1.9574 e-09$ \\
\hline & CPU-time (sec) & 106.188 & 929.467 \\
\hline
\end{tabular}

Table 5.1: European spread option price calculated by the proposed method (FDM) and Analytical approximation 5.44 .

Furthermore, unsuitable negative values appear in analytical approximation for small $S_{1}$ and $S_{2}$ while the proposed method preserves non-negativity of the solution.

Finally, in Example 5.2.3 we compare the numerical solution obtained with our scheme (5.42) using LCP approach with other tested efficient methods presented in [34].

Example 5.2.3. Let us consider the American spread call option problem with parameters:

$$
T=1 ; \sigma_{1}=0.1 ; \sigma_{2}=0.2 ; r=0.1 ; q_{1}=0.05 ; q_{2}=0.05 ; \rho=0.5 .
$$

Table 5.2 shows the option price at fixed asset prices $S_{1}=96$ and $S_{2}=100$ for different values of strike price $E$ evaluated by one dimensional integration analytic method (Analytic), Fast Fourier Transform (FFT), Monte Carlo method (MC) and our proposed method. The accuracy is competitive with other methods, such as Fourier Transform with high number of discretization (4096) and Monte Carlo with big number of time steps (2000).

Another set of parameters can be considered in order to compare it with data in [118]:

$$
\begin{aligned}
& T=0.5 ; E=100 ; \sigma_{1}=0.25 ; \sigma_{2}=0.3 ; \\
& r=0.03 ; q_{1}=0.06 ; q_{2}=0.02 ; \rho=0.5 .
\end{aligned}
$$




\begin{tabular}{|c|c|c|c|c|}
\hline E & Analytic & FFT & MC & Proposed Method \\
\hline 0 & 8.513201 & 8.513079 & 8.516613 & 8.508198 \\
2 & 7.542296 & 7.542242 & 7.545496 & 7.534064 \\
4 & 6.653060 & 6.652976 & 6.656364 & 6.648792 \\
\hline
\end{tabular}

Table 5.2: American spread option price calculated by various methods for $E=$ $0,2,4$.

\begin{tabular}{|c|c|c|c|c|}
\hline$N_{\tau}$ & NIM & MOL & Monte Carlo & Proposed method \\
\hline 50 & 296.598 & 59.20 & 47970 & 26.547 \\
& 0.003 & 0.001 & 0.1735 & 0.0036 \\
\hline 32 & 127.069 & 55.12 & 1811 & 17.911 \\
& 0.0035 & 0.006 & 0.1148 & 0.0122 \\
\hline 16 & 33.493 & 51.718 & 5149 & 9.432 \\
& 0.0012 & 0.0236 & 0.132 & 0.0371 \\
\hline 8 & 10.360 & 49.01 & 1350 & 4.787 \\
& 0.0105 & 0.074 & 0.1316 & 0.0907 \\
\hline 4 & 4.117 & 45.47 & 355.75 & 2.815 \\
& 0.0364 & 0.2512 & 0.1351 & 0.2254 \\
\hline
\end{tabular}

Table 5.3: CPU-Time in sec (first row) and Absolute difference (second row) for different methods depending on number of time-steps for fixed number of space steps for parameters 5.45 .

Table 5.3 presents a comparison of the efficiency of the proposed method with method of lines (MOL), numerical integration method (NIM) and Monte-Carlo. In [118] author fixes $S_{1}=200, S_{2}=100$ to calculate the absolute difference. From the Table 5.2 we can state that the proposed method has the same order of accuracy, but it requires less computational time.

The results of this section have been submitted to Journal of Applied Mathematics and Computation. 



\section{Conclusions}

The front-fixing method is a very useful technique dealing with free boundary value problems, such as American option pricing. Various transformations have been applied to linear Black-Scholes equation. The resulting nonlinear PDEs have been solved by explicit FDM. Stability and consistency of the proposed schemes have been studied as well as positivity and monotonicity of the numerical solution.

The front-fixing method has been extended to nonlinear equations and regime switching models of American option pricing. The system of coupled nonlinear equations has been solved by explicit method that avoids amount of iterations. Von Neumann stability analysis has been used to find stability conditions of the scheme.

In the case of nonlinear Black-Scholes models, such as Barles and Soner or RAPM, instead of front-fixing technique a new moving-boundary transformation has been applied. The resulting equation is established in a domain with timedependent boundary. Various FMDs are used for numerical solutions, including fully implicit and ADE methods. Improvements of Broyden's method have been proposed as well.

Theoretical study of behavioural modelling of option pricing has been completed by numerical solution and analysis of the proposed method. This new approach has been successfully applied to regime switching model. In this case we take into account possible states of markets as well as irrational exercise possibility. Numerical solution of nonlinear coupled system has been found by using $\theta$-method. Qualitative properties of the proposed scheme have been studied. 
The suitable change of variables in multi-asset case allows to remove the cross derivative term. Effective FDM has been constructed for the resulting equation in the canonic form. The elimination of mixed derivative simplifies the stencil of FDM and numerical analysis of the scheme.

All the theoretical results have been approved by numerical experiments. $\mathrm{Nu}$ merical examples are provided in order to show the efficiency of the methods and to prove its competitiveness by comparing with other recognized approaches in the literature. These numerical schemes provide positive, consistent and conditionally stable solutions. 


\section{References}

[1] C. Alexander and A. Venkatramanan. Analytic approximations for spread options. Technical report, ICMA Centre Discussion Papers in Finance DP2007-11, 2007. 137, 150, 151

[2] J. Ankudinova and M. Ehrhardt. On the numerical solution of nonlinear Black-Scholes equations. Computers \& Mathematics with Applications, 56(3):799 - 812, 2008. Mathematical Models in Life Sciences \& Engineering. 59, 62, 79

[3] K. Atkinson. An Introduction to Numerical Analysis. John Wiley \& Sons, 2 edition, 1989. 8

[4] M. Avellaneda, A. Lévy, and A. Parás. Pricing and hedging derivative securities in markets with uncertain volatilities. Applied Mathematical Finance, 2(2):73-88, 1995. 57

[5] J. Babbin, P. Forsyth, and G. Labahn. A comparison of iterated optimal stopping and local policy iteration for American options under regime switching. Journal of Scientific Computing, 58(2):409-430, 2014. 90, 92

[6] G. Barles and H. M. Soner. Option pricing with transaction costs and a nonlinear Black-Scholes equation. Finance and Stochastics, 2(4):369-397, 1998. 2, 57, 60

[7] G. Barone-Adesi and R. Whaley. Efficient analytic approximation of American option values. The Journal of Finance, 42(2):301-320, 1987. 1, 40. 52

[8] D. Bertsekas. Convex Analysis and Optimization. Athena Scientific, 2003. 34,47 
[9] M. Bierbrauer, S. Trueck, and R. Weron. Modeling electricity prices with regime switching models. Econometrics 0502005, EconWPA, Feb. 2005. 58

[10] F. Black and M. Scholes. The pricing of options and corporate liabilities. Journal of Political Economy, 81(3):637-654, 1973. 2, 57, 58

[11] A. Borici and H. Lüthi. Fast solutions of complementarity formulations in American put pricing. 9:63-82, 2005. 1

[12] P. P. Boyle. Options: A Monte-Carlo approach. Journal of Financial Economics, 4(3):323 - 338, 1977. 137

[13] P. P. Boyle. A lattice framework for option pricing with two state variables. Journal of Financial and Quantitative Analysis, 23(01):1-12, 1988. 137

[14] P. P. Boyle and T. Vorst. Option replication in discrete time with transaction costs. The Journal of Finance, 47(1):271-293, 1992. 2, 57

[15] P. Bremaud. Point Processes and Queues: Martingale Dynamics. Springer Series in Statistics. Springer New York, 1981. 99

[16] M. Brennan and E. Schwartz. Finite difference methods and jump processes arising in the pricing of contingent claims: a synthesis. Journal of Financial and Quantitative Analysis, 1(3):461-474, 1978. 2, 117

[17] M. Broadie and J. Detemple. American option valuation: approximations and a comparison of existing methods. Review of Financial Studies, 9:12111250, 1996. 39

[18] J. Y. Campbell, A. W. Lo, and A. C. MacKinlay. The econometrics of financial markets. Princeton University Press, Princeton, NJ, USA, 1997. 4

[19] R. Carmona and V. Durrleman. Pricing and hedging spread options. SIAM Review, 45(4):627-685, 2003. 137, 141

[20] S. Chen and M. Insley. Regime switching in stochastic models of commodity prices: An application to an optimal tree harvesting problem. Journal of Economic Dynamics and Control, 36(2):201 - 219, 2012. 58

[21] Z. Chen and P. A. Forsyth. Implications of a regime-switching model on natural gas storage valuation and optimal operation. Quantitative Finance, 10(2):159-176, 2010. 58 
[22] C. Chiarella, B. Kang, G. Mayer, and A. Ziogas. The evaluation of American option prices under stochastic volatility and jump-diffusion dynamics using the method of lines. International Journal of Theoretical and Applied Finance, 12(03):393-425, 2009. 138

[23] C. Chiarella and J. Ziveyi. Pricing American options written on two underlying assets. Quantitative Finance, 14(3):409-426, 2014. 137

[24] R. Company, V. Egorova, and L. Jódar. Constructing positive reliable numerical solution for American call options: A new front-fixing approach. Journal of Computational and Applied Mathematics, 291:422 - 431, 2016. Mathematical Modeling and Computational Methods. 54, 59, 79, 117

[25] R. Company, V. Egorova, L. Jódar, and C. Vázquez. Finite difference methods for pricing american put option with rationality parameter: Numerical analysis and computing. Journal of Computational and Applied Mathematics, 304:1-17, 2016. 120, 132

[26] R. Company, V. N. Egorova, and L. Jódar. Solving American option pricing models by the front-fixing method: Numerical analysis and computing. Abstract and Applied Analysis, Article ID 146745, 9 pages, 2014. 28, 79. 138

[27] R. Company, V. N. Egorova, L. Jódar, and C. Vázquez. Computing American option price under regime switching with rationality parameter. Journal of Computers \& Mathematics with Applications, 2016, in press. 136

[28] R. Company, L. Jódar, and J. R. Pintos. Consistent stable difference schemes for nonlinear Black-Scholes equations modelling option pricing with transaction costs. ESAIM: Mathematical Modelling and Numerical Analysis, 43(6):1045-1061, 6 2009. 58, 61

[29] R. Company, L. Jódar, E. Ponsoda, and C. Ballester. Numerical analysis and simulation of option pricing problems modeling illiquid markets. Computers \& Mathematics with Applications, 59(8):2964 - 2975, 2010. 66

[30] R. Company, L. Jódar, and J.-R. Pintos. A consistent stable numerical scheme for a nonlinear option pricing model in illiquid markets. In Mathematics and Computers in Simulations, volume 82, pages 1972-1985. 2012. 50 
[31] R. Company, E. Navarro, J. R. Pintos, and E. Ponsoda. Numerical solution of linear and nonlinear Black-Scholes option pricing equations. Computers \& Mathematics with Applications, 56(3):813 - 821, 2008. Mathematical Models in Life Sciences \&amp; Engineering. 61, 62

[32] S. Conte and C. W. de Boor. Elementary Numerical Analysis: An Algorithmic Approach. McGraw-Hill Higher Education, 3rd edition, 1980. 11, 88

[33] J. Crank. Free and Moving Boundary problems. Oxford University Press, 1984. 2, 58, 79

[34] M. Dempster and S. Hong. Spread option valuation and the fast fourier transform. Technical report, Judge Institute of Management Studies, University of Cambridge, 2000. 152

[35] M. Dempster, J. Hutton, and D. D.G. Richards. LP valuation of exotic American options exploiting structure. Journal Of Computational Finance, 2:61-84, 1998. 1

[36] F. Diz and T. J. Finucane. The rationality of early exercise decisions: Evidence from the S\&P 100 index options market. The Review of Financial Studies, 6(4):765-797, 1993. 97

[37] D. Duffy. Finite difference methods in financial engineering : A partial differential equation approach. John Wiley and Sons Ltd, UK, 2006. 2, 141

[38] D. Duffy. Unconditionally stable and second-order accurate explicit finite difference schemes using domain transformation: Part I one-factor equity problems. Technical report, SSRN, 2009. Available at SSRN: http://ssrn.com/abstract=1552926. 59

[39] B. Düring and M. Fournié. High-order compact finite difference scheme for option pricing in stochastic volatility models. Journal of Computational and Applied Mathematics, 236(17):4462-4473, 2012. 86, 127

[40] B. Düring, M. Fournié, and C. Heuer. High-order compact finite difference schemes for option pricing in stochastic volatility models on non-uniform grids. Journal of Computational and Applied Mathematics, 271:247 - 266, 2014. 138 
[41] B. Düring, M. Fournié, and A. Jüngel. Convergence of a high-order compact finite difference scheme for a nonlinear Black-Scholes equation. ESAIM: Mathematical Modelling and Numerical Analysis - Modélisation Mathématique et Analyse Numérique, 38(2):359-369, 2004. 58

[42] V. Egorova, S.-H. Tan, C.-H. Lai, R. Company, and L. Jódar. Moving boundary transformation for american call options with transaction cost: finite difference methods and computing. International Journal of Computer Mathematics, pages 1-18, 2015. Published online: 08 Dec 2015. 75

[43] V. N. Egorova, R. Company, and L. Jódar. A new efficient numerical method for solving American option under regime switching model. Computers \& Mathematics with Applications, 71(1):224 - 237, 2016. 94, 132

[44] M. Ehrhardt. Discrete artificial boundary conditions. PhD thesis, Technische Universitat Berlin, 2001. 143

[45] R. J. Elliott, T. K. Siu, L. Chan, and J. W. Lau. Pricing options under a generalized Markov-modulated jump-diffusion model. Stochastic Analysis and Applications, 25(4):821-843, 2007. 78

[46] J. Evans, R. Kuske, and J. Keller. American options on assets with dividends near expiry, 2002. 39

[47] M. Fakharany, R. Company, and L. Jódar. Positive finite difference schemes for a partial integro-differential option pricing model. Applied Mathematics and Computation, 249:320 - 332, 2014. 138

[48] P. Forsyth and K. Vetzal. Quadratic convergence of a penalty method for valuing American options, 2002. 2, 117, 149

[49] R. Frontczak and R. Schöbel. On modified Mellin transforms, GaussLaguerre quadrature, and the valuation of American call options. Journal of Computational and Applied Mathematics, 234:1559-1571, 2010. 39

[50] K. S. T. Gad and J. L. Pedersen. Rationality parameter for exercising American put. Risks, 3(2):103, 2015. 97, 98, 99, 100, 115, 132

[51] P. R. Garabedian. Partial Differential Equations. AMS Chelsea Publishing, 1998. 142 


\section{REFERENCES}

[52] R. Geske and H. Johnson. The American put option valued analytically. Journal of Finance, 39 (5):1511-1524, 1984. 1

[53] B. Gustafsson, H. O. Kreiss, and J. Oliger. Time-Dependent Problems and Difference Methods, Second Edition. John Wiley \& Sons, Inc., 2013. 86. 127

[54] H. Han and X. Wu. A fast numerical method for the Black-Scholes equation of American options. SIAM Journal on Numerical Analysis, 41(6):20812095, 2003. 28, 39, 41, 51, 117

[55] M. R. Hardy. A regime-switching model of long-term stock returns. North American Actuarial Journal, 5(2):41-53, 2001. 58

[56] P. Heider. Numerical methods for nonlinear Black-Scholes equations. Applied Mathematical Finance, 17(1):59-81, 2010. 58

[57] Y. Huang, P. A. Forsyth, and G. Labahn. Methods for pricing American options under regime switching. SIAM Journal on Scientific Computing, 33(5):2144-2168, 2011. 58,79

[58] J. Hull. Options, Futures and Other Derivatives. Pearson/Prentice Hall, 2009. 3, 5, 93,148

[59] J. Hull and A. White. Valuing derivative securities using the explicit finite difference method. Journal of Financial and Quantitative Analysis, 25(1):87-100, 1990. 2, 16,60

[60] S. Ikonen and J. Toivanen. Operator splitting methods for American option pricing. Applied Mathematics Letters, 17(7):809 - 814, 2004. 28, 39

[61] K. int Hout and C. Mishra. Stability of ADI schemes for multidimensional diffusion equations with mixed derivative terms. Applied Numerical Mathematics, 74:83 - 94, 2013. 138

[62] P. Jaillet, D. Lamberton, and B. Lapeyere. Variational inequalities and the pricing of American options. Acta Applicandae Mathematicae, 21:263-289, 1990. 2

[63] M. Jandačka and D. Ševčovič. On the risk-adjusted pricing-methodologybased valuation of vanilla options and explanation of the volatility smile. Journal of Applied Mathematics, 2005(3):235-258, 2005. 2, 57, 73 
[64] C. Joy, P. P. Boyle, and K. S. Tan. Quasi-Monte Carlo methods in numerical finance. Management Science, 42(6):926-938, 1996. 137

[65] N. Ju. Pricing an american option by approximating its early exercise boundary as a multipiece exponential function. In Review of Financial Studies, volume 11(3), pages 627-646. 1998. 1

[66] R. Kangro and R. Nicolaides. Far field boundary conditions for BlackScholes equations. SIAM Journal on Numerical Analysis, 38(4):1357-1368, 2000. 17, 29, 81, 103, 143, 147

[67] W. H. Kao, Y. D. Lyuu, and K. W. Wen. The hexanomial lattice for pricing multi-asset options. Applied Mathematics and Computation, 233:463 - 479, 2014. 137

[68] I. Karatzas and S. Shreve. Methods of Mathematical Finance. Applications of Mathematics: Stochastic Modelling and Applied Probability. Springer, 1998. 101

[69] C. Kelley. Iterative Methods for Linear and Nonlinear Equations. Society for Industrial and Applied Mathematics, 1995. 45

[70] A. Khaliq, B. Kleefeld, and R. Liu. Solving complex PDE systems for pricing American options with regime-switching by efficient exponential time differencing schemes. Numerical Methods for Partial Differential Equations, 29(1):320-336, 2013. 79, 89, 91, 93, 94, 132

[71] A. Q. M. Khaliq and R. H. Liu. New numerical scheme for pricing American option with regime-switching. International Journal of Theoretical and Applied Finance, 12(03):319-340, 2009. 79, 92, 123

[72] I. J. Kim. The analytic valuation of American options. The Review of Financial Studies, 3:547-572, 1990. 35, 49

[73] E. Kirk. Correlation in the Energy Markets, chapter Managing Energy Price Risk, pages 71-78. Risk Publications and Enron, London, 1995. 137

[74] D. Knuth. The Art of Computer Programming 3. Addison-Wesley, 1997. 63

[75] M. Kratka. No mystery behind the smile. Risk, 9:67-71, 1998. 2, 57 
[76] D. Kröner. Numerical Schemes for Conservation Laws. Wiley and Teubner, 1997. 13

[77] Y. K. Kwok. Mathematical Models of Financial Derivatives. Springer, 2008. 59.79

[78] C.-H. Lai. An application of quasi-Newton methods for the numerical solution of interface problems. Advances in Engineering Software, 28(5):333 339, 1997. 11, 45, 59, 66

[79] H. Landau. Heat conduction in a melting solid. Quarterly Applied Mathematics, 8:81-95, 1950. 17, 61,79

[80] D. Lando. On Cox processes and credit risky securities. Review of Derivatives Research, 2(2-3):99-120, 1998. 101

[81] D. P. J. Leisen and M. Reimer. Binomial models for option valuation examining and improving convergence. Applied Mathematical Finance, 3(4):319-346, 1996. 117

[82] H. Leland. Option pricing and replication with transactions costs. The Journal of Finance, 40(5):1283-1301, 1985. 2, 57

[83] D. C. Lesmana and S. Wang. An upwind finite difference method for a nonlinear Black-Scholes equation governing European option valuation under transaction costs. Applied Mathematics and Computation, 219(16):8811 $8828,2013.58$

[84] S. Leung and S. Osher. An alternating direction explicit (ADE) scheme for time-dependent evolution equations. Technical report, UCLA, 2005. 66

[85] G. Liu. Mesh free methods: moving beyond the finite element method. CRC Press, 2002. 71

[86] H. K. Liu. The Valuation of American Options on Single Asset and Multiple Assets. PhD thesis, National Chengchi University, Taiwan, 2007. Xvi, 138, 139,140

[87] R. H. Liu. Regime-switching recombining tree for option pricing. International Journal of Theoretical and Applied Finance, 13(03):479-499, 2010. 78, 89, 132 
[88] E. Marwil. Convergence results for Schubert's method for solving sparse nonlinear equations. SIAM Journal on Numerical Analysis, 16(4):588-604, 1979. 70

[89] K. Muthuraman. A moving boundary approach to American option pricing. Journal of Economic Dynamics and Control, 32(11):3520 - 3537, 2008. 28

[90] B. Nielsen, O. Skavhaug, and A. Tvelto. Penalty and front-fixing methods for the numerical solution of American option problems. Journal Of Computational Finance, 5, 2002. 2, 25, 27, 28, 59, 79

[91] R. Panini and R. Srivastav. Option pricing with Mellin transnforms. Mathematical and Computer Modelling, 40(1-2):43 - 56, 2004. 27

[92] G. Pealat and D. Duffy. The alternating direction explicit (ADE) method for one-factor problems. Wilmott magazine, 2011(54):54-60, 2011. 59

[93] R. Plemmons. M-matrix characterizations. I-nonsingular M-matrices. Linear Algebra and its Applications, 18(2):175 - 188, 1977. 122, 127

[94] D. M. Pooley, P. A. Forsyth, and K. R. Vetzal. Numerical convergence properties of option pricing PDEs with uncertain volatility. IMA Journal of $\mathrm{Nu}$ merical Analysis, 23, 2003. 58

[95] A. M. Poteshman and V. Serbin. Clearly irrational financial market behavior: Evidence from the early exercise of exchange traded stock options. The Journal of Finance, 58(1):37-70, 2003. 97

[96] A. Saib, Y. Tangman, N. Thakoor, and M. Bhuruth. On some finite difference algorithms for pricing American options and their implementation in Mathematica. In Proceedings of the 11th International Conference on Computational and Mathematical Methods in Science and Engineering. 2011. 26, 28, 39

[97] S. Salmi, J. Toivanen, and L. von Sydow. Iterative methods for pricing American options under the Bates model. Procedia Computer Science, 18:1136 1144, 2013. 2013 International Conference on Computational Science. 138

[98] A. A. Samarskii. The Theory of Difference Schemes. Marcel Dekker, Inc, New-York, 2001. 7, 10, 11 


\section{REFERENCES}

[99] P. Samuelson. Rational theory of warrant pricing. Industrial Management Review, 6(2):13-39, 1965. 22

[100] R. U. Seydel. Tools for Computational Finance. Springer London, 2012. 142

[101] G. D. Smith. Numerical Solution of Partial Differential Equations: Finite Difference Methods, 3rd ed. Clarendon Press, Oxford, 1985. 11, 13, 25, 83, 86, 121, 127, 129, 130, 131

[102] J. Strikwerda. Finite Difference Schemes and Partial Differential Equations, Second Edition. Society for Industrial and Applied Mathematics, 2004. 11. 13, 86, 127, 129

[103] D. Tangman, A. Gopaul, and M. Bhuruth. A fast high-order finite difference algorithm for pricing American options. Journal of Computational and Applied Mathematics, 222(1):17 - 29, 2008. Special Issue: Numerical PDE Methods in Finance. 2, 113,117

[104] D. Tavella and C. Randall. Pricing Financial Instruments: The Finite Difference Method. John Wiley and Sons, New York, 2007. 2, 138

[105] J. Toivanen. A componentwise splitting method for pricing American options under the Bates model. In W. Fitzgibbon, Y. Kuznetsov, P. Neittaanmäki, J. Périaux, and O. Pironneau, editors, Applied and Numerical Partial Differential Equations, volume 15 of Computational Methods in Applied Sciences, pages 213-227. Springer Netherlands, 2010. 138

[106] J. Toivanen. Finite difference methods for early exercise options. In Encyclopedia of Quantitative Finance. John Wiley \& Sons, Ltd, 2010. 28, 81

[107] D. Ševčovič. Analysis of the free boundary for the pricing of an American call option. European Journal of Applied Mathematics, null:25-37, 22001. 59

[108] D. Ševčovič. An iterative algorithm for evaluating approximations to the optimal exercise boundary for a nonlinear Black-Scholes equation. Canadian Applied Mathematics Quarterly, 15(1):77-97, 2007. 59, 62,79 
[109] D. Ševčovič. Transformation methods for evaluating approximations to the optimal exercise boundary for linear and nonlinear Black-Scholes equations. In Nonlinear Models in Mathematical Finance: New Research Trends in Option Pricing, pages 173-218. Nova Science Publishers,Inc., New York, 2008. xv, 2, 39, 40, 52, 53

[110] P. Wilmott, T. Hoggard, and A. E. Whalley. Hedging option portfolios in the presence of transaction costs. Advances in futures and option research, 7:217-235, 1994. 57

[111] P. Wilmott, S. Howison, and J. Dewynne. The Mathematics of Financial Derivatives. Cambridge University Press, Cambridge, UK, 1995. 2, 3, 16 , 43,60

[112] L. Wu and Y.-K. Kwok. A front-fixing method for the valuation of American option. 6:83-97, 1997. 2, 18, 22, 29, 43, 58,79

[113] G. G. Yin and Q. Zhang. Continuous-time Markov Chains and Applications: A Singular Perturbation Approach. Springer-Verlag New York, Inc., New York, NY, USA, 1998. 78

[114] F. L. Yuen and H. Yang. Option pricing with regime switching by trinomial tree method. Journal of Computational and Applied Mathematics, 233(8): $1821-1833,2010.78$

[115] J. Zhang and S. Zhu. A hybrid finite difference method for valuing American puts. In Proceedings of the World Congress of Engineering, volume II. London, UK, 2009. 2, 18

[116] K. Zhang, K. Teo, and M. Swartz. A robust numerical scheme for pricing American options under regime switching based on penalty method. Computational Economics, 43(4):463-483, 2014. 79, 90, 92, 93

[117] Q. Zhang and X. Y. Zhou. Valuation of stock loans with regime switching. SIAM Journal on Control and Optimization, 48(3):1229-1250, 2009. 58

[118] J. Ziveyi. The Evaluation of Early Exercise Exotic Options. PhD thesis, University of Technology, Sydney, Australia, 2011. 137, 152, 153 


\section{Declaration}

I herewith declare that I have produced this work without the prohibited assistance of third parties and without making use of aids other than those specified; notions taken over directly or indirectly from other sources have been identified as such. This work has not previously been presented in identical or similar form to any examination board.

The dissertation work was conducted from 2013 to 2016 under the supervision of Dr. Lucas Jódar Sánchez and Dr. Rafael Company Rossi at the Polytechnic University of Valencia.

Valencia, 
This dissertation was finished writing in Valencia on April 21, 2016 
This page is intentionally left blank 